Karl Christoph Heinen

\title{
Die Berücksichtigung von Kosten in der \\ Konkurrenzanalyse
}


Karl Christoph Heinen

\section{Die Berücksichtigung von Kosten in der Konkurrenzanalyse}

In dieser Arbeit wird die Bedeutung von Kosten untersucht, die im Rahmen einer Konkurrenzanalyse von Wettbewerbsunternehmen erfasst werden können. Der Autor stellt zunächst unterschiedliche Instrumente und Quellen vor, aus denen Informationen über Konkurrenten zu gewinnen sind. Die Bedeutung von Kosteninformationen über Wettbewerber wird empirisch mit Hilfe des Unternehmensplanspiels MARGA untersucht. Die Ergebnisse zeigen, dass Unternehmen, die über eigene Kostenvorteile im Vergleich zu ihren Wettbewerbern informiert sind, ein anderes strategisches Entscheidungsverhalten zeigen. Sie sind wirtschaftlich erfolgreicher, als Unternehmen, die ihre Kostenvorteile nicht kennen. Abschließend wird daher die Berücksichtigung von Kosten in der Konkurrenzanalyse ausdrücklich empfohlen.

Karl Christoph Heinen, geboren 1967, studierte Wirtschafts- und Sozialwissenschaften an der Universität Fribourg/Schweiz. Anschließend war er als Wissenschaftlicher Mitarbeiter am Universitätsseminar der Wirtschaft Schloss Gracht beschäftigt und leitet dort das betriebswirtschaftliche Planspiel MARGA. Darüber hinaus ist er als Lehrbeauftragter an der FH Magdeburg tätig. Die Promotion erfolgte 2001. 
Die Berücksichtigung von Kosten in der Konkurrenzanalyse 


\section{Beiträge zum Controlling}

Herausgegeben von Wolfgang Berens

Band 3

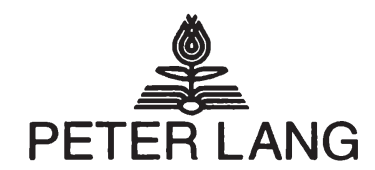

Frankfurt am Main - Bertin - Bern - Bruxelles - New York · Oxford - Wien 


\section{Karl Christoph Heinen}

\section{Die Berücksichtigung von Kosten in der Konkurrenzanalyse}

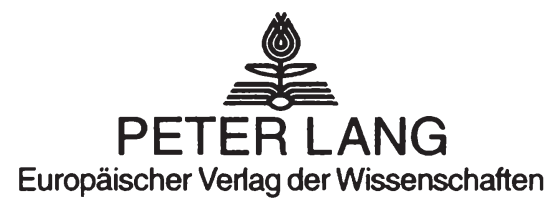

Christoph Heinen - 978-3-631-75315-6

Downloaded from PubFactory at 01/11/2019 06:18:34AM

via free access 
Die Deutsche Bibliothek - CIP-Einheitsaufnahme

Heinen, Karl Christoph:

Die Berücksichtigung von Kosten in der Konkurrenzanalyse /

Karl Christoph Heinen. - Frankfurt am Main ; Berlin ; Bern ;

Bruxelles ; New York ; Oxford ; Wien : Lang, 2002

(Beiträge zum Controlling; Bd. 3)

Zugl.: Düsseldorf, Univ., Diss., 2001

ISBN3-631-39158-7

Open Access: The online version of this publication is published on www.peterlang.com and www.econstor.eu under the

international Creative Commons License CC-BY 4.0. Learn more on how you can use and share this work: http://creativecommons. org/licenses/by/4.0.

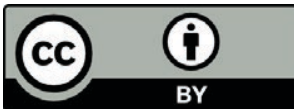

This book is available Open Access thanks to the kind support of ZBW - Leibniz-Informationszentrum Wirtschaft.

Gedruckt auf alterungsbestăndigem, säurefreiem Papier.

\author{
D61 \\ ISSN 1618-825X \\ ISBN3-631-39158-7 \\ ISBN 978-3-631-75315-6 (eBook) \\ (C) Peter Lang GmbH \\ Europäischer Verlag der Wissenschaften \\ Frankfurt am Main 2002 \\ Alle Rechte vorbehalten.
}

Das Werk einschließlich aller seiner Teile ist urheberrechtlich geschützt. Jede Verwertung außerhalb der engen Grenzen des Urheberrechtsgesetzes ist ohne Zustimmung des Verlages unzulässig und strafbar. Das gilt insbesondere für Vervielfältigungen, Übersetzungen, Mikroverfilmungen und die Einspeicherung und Verarbeitung in elektronischen Systemen.

Printed in Germany 123467

www.peterlang.de 


\section{Geleitwort}

Die Konkurrenzanalyse gewinnt an Bedeutung, je intensiver Unternehmen im Wettbewerb zueinander stehen. In der vorliegenden Arbeit stellt der Verfasser die Frage nach der Wichtigkeit von Kosteninformationen, welche im Zuge einer systematischen Konkurrenzanalyse über Wettbewerbsunternehmen gewonnen werden können. Derartige Informationen fließen in den strategischen Entscheidungsprozess von Unternehmen ein, da Kosten im Allgemeinen nicht nur eine ergebnisrelevante, sondern auch eine strategiebeeinflussende Bedeutung haben.

Zur Untersuchung der aufgeworfenen Fragestellung reicht eine theoretische Abhandlung nicht aus. Vielmehr muss das tatsächliche Verhalten von Entscheidungsträgern in konkreten Wettbewerbssituationen beobachtet und empirisch ausgewertet werden. Hierzu bietet sich die Nutzung eines Unternehmensplanspiels im Rahmen eines experimentellen Forschungsprojektes an.

Als Leiter des Unternehmensplanspiels MARGA steht dem Verfasser ein entsprechendes, seit vielen Jahren etabliertes Instrument zur Verfügung. Für die zu untersuchende Problematik konnte dadurch sehr zielgerichtet umfangreiches empirisches Material gewonnen und ausgewertet werden.

Insgesamt stellt die vorliegende Dissertationsschrift eine theoretisch fundierte und empirisch belegte Arbeit dar. Herrn Heinen gelingt zudem der Brückenschlag zwischen Theorie und Praxis. Sehr anschaulich erläutert er die strategische Bedeutung einer systematischen Konkurrenzanalyse und spricht der regelmäßigen Berücksichtigung von Kosteninformationen in der Konkurrenzanalyse eine hohe Bedeutung zu. Die Arbeit stellt darüber hinaus eine hervorragende Einführung in das Themengebiet der Konkurrenzanalyse dar.

Insgesamt wünsche ich Herrn Heinen eine weite Verbreitung seiner Arbeit in der betriebswirtschaftlichen Theorie und in der unternehmerischen Praxis.

Professor Dr. Wolfgang Berens 
Christoph Heinen - 978-3-631-75315-6

Downloaded from PubFactory at 01/11/2019 06:18:34AM

via free access 


\section{Vorwort}

Eine Doktorarbeit entsteht in einem - schon sprichwörtlich - langwierigen Prozess, der geprägt ist durch unzählige Erlebnisse, Rückschläge und Erfolge. Diese zusammengenommen machen für mich den persönlichen Wert einer derartigen Arbeit aus.

In meinem Umfeld gab es viele Menschen, die diesen Entstehungsprozess begleitet und unterstützt haben und denen ich für ihren Teil danke.

An erster Stelle möchte ich meinen Doktorvater, Herm Professor Dr. WolfGaNG BERENS, anführen, der durch seine Bereitschaft, mich als externen Doktoranden zu betreuen, diese Arbeit erst ermöglicht hat. Ich danke ihm als "Doktorsohn“ für seine fachliche und persönliche Unterstützung und für die gleichzeitige Freiheit, die er mir gewährt hat. Persönlich danke ich ihm für unsere freundschaftliche Zusammenarbeit.

Ich danke den Mitarbeitern des Lehrstuhls für Betriebswirtschaftslehre, insbesondere Controlling, der HEINRICH-HEINE-UNIVERSITÄT DüSSELDORF bZW. der WESTFÄLISCHEN WILHELMS-UNIVERSITÄT MÜNSTER für viele Gespräche und Anregungen. Hier möchte ich besonders Herm Dr. ANDREAS HOFFJAN erwähnen, der mir von Beginn an bis zur Fertigstellung der Dissertationsschrift stets als wissenschaftlich kompetenter Gesprächspartner zur Seite stand und der mir somit die Weiterentwicklung meiner Ideen ermöglichte. Darüber hinaus danke ich Herm Dr. WALTER SCHMITTING, der besonders in der Phase der Konzepterstellung und während der Vorbereitung auf das Rigorosum wichtige Ratschläge gegeben hat.

Mein Dank gilt dem Zweitgutachter der vorliegenden Arbeit, Herm Professor Dr. KLAUS-PETER Franz, mit dem mich eine gemeinsame Zeit am UNIVERSITÄTSSEMINAR DER WIRTSCHAFT, USW, Schloss Gracht, verbindet. Aufgrund seiner damaligen Funktion als wissenschaftlicher Direktor hat er einen engen Bezug zu dem in der vorliegenden Arbeit verwendeten Instrumentarium, dem Planspiel MARGA.

Die vorliegende Arbeit habe ich berufsbegleitend, neben meiner Vollzeittätigkeit als wissenschaftlicher Mitarbeiter, Dozent und Leiter des Unternehmensplan- 
spiels MARGA verfasst. Dem USW danke ich für diese Möglichkeit. Große Unterstützung erfuhr ich bei meinen „Planspiel-Kollegen“: Dr. THOMAS WERNIUK, mit dessen Hilfe die elektronische Erfassung des umfangreichen Datenmaterials gelang, sowie ANDREAS NILL, der mich oft fachlich vertreten hat. Hervorzuheben sind auch VERONIKA KUNOW und BRIGITTE ROYE, die mich bei vielen organisatorischen Aufgaben des meist parallel laufenden Planspielwettbewerbs entlasten konnten.

Mein ganz besonderer Dank gilt meiner Familie und meinen Freunden. Ich bedanke mich für euer Verständnis, denn ich konnte nicht viel Zeit mit euch verbringen. Das Ergebnis haltet ihr in den Händen.

Zwei Menschen haben einen herausragenden Anteil am Entstehungsprozess dieser Arbeit: Mein Großvater, Franz Heuser, und meine Frau Britta. Mein Großvater hat nach dem Studium in mir Interesse an einer Promotion geweckt und diese damit initiiert. Meine Frau Britta hat mich auf dem langen Weg so intensiv wie niemand sonst begleitet. Mit ihr habe ich Zweifel besprochen, Rückschläge verarbeitet und Erfolge gefeiert, sie hat mich motiviert und immer unterstützt. Beiden sei diese Arbeit gewidmet.

K. Christoph Heinen 


\section{Inhaltsverzeichnis}

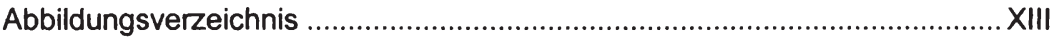

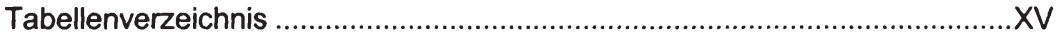

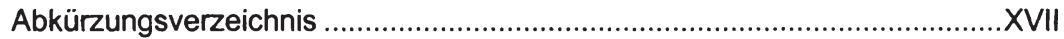

Problemstellung, Zielsetzung und Aufbau der Arbeit .................................... 1

Teil 1 Die Konkurrenzanalyse als Teil der strategischen Planung............ 5

1. Kapitel Strategischer Planungsprozess und Einordnung der Konkurrenz-

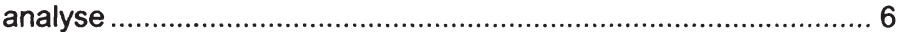

I.1.1 Definitionen und Merkmale der strategischen Unternehmensplanung

I.1.2 Präskriptiv-synoptischer und deskriptiv-inkrementaler Prozessansatz der strategischen Planung ......................... 8

I.1.3 Ablauf- und Prozessmodelle der strategischen Planung.. 10

I.1.3.1 Phase der Zielbildung ........................................ 16

I.1.3.2 Phase der strategischen Analyse ...................... 17

I.1.3.3 Phase der Strategieformulierung ........................ 26

1.1.3.4 Phase der Strategieumsetzung ......................... 32

2. Kapitel Definition und Abgrenzung der Konkurrenzanalyse....................... 34

1.2.1 Definition der Konkurrenzanalyse .................................... 34

1.2.2 Abgrenzung der Konkurrenzanalyse von verwandten analytischen Ansätzen .................................................. 37

3. Kapitel Inhalte und Instrumente der Konkurrenzanalyse .......................... 40

1.3.1 Stärken-/Schwächen-Analyse und Konkurrenzanalyse.... 41

1.3.2 Checklisten in der Konkurrenzanalyse ............................ 43

1.3.3 Bilanzanalyse im Rahmen der Konkurrenzanalyse ......... 47

1.3.4 System zur Konkurrentenanlyse nach PORTER ................ 52

1.3.5 Erfahrungskurve als Instrument der Konkurrenzanalyse.. 55

1.3.6 Wertkette in der Konkurrenzanalyse .............................. 59

1.3.7 Portfolio-Analyse in der Konkyrrenzanalyse 
4. Kapitel Informationsgewinnung in der Konkurrenzanalyse

1.4.1 Begriff der Information und Darstellung des betrieblichen Informationssystems ................................... 71

I.4.2 Informationsquellen in der Konkurrenzanalyse .............. 74

I.4.3 Gewinnung von Kosteninformationen von Wettbewerbsunternehmen

5. Kapitel Kostenbetrachtungen in der Konkurrenzanalyse 84

I.5.1 Unterschiedliche Bedeutung von Kosteninformationen in der Konkurrenzanalyse im Schrifttum....................... 85

I.5.2 Untersuchung der Bedeutung von Kosteninformationen in der Konkurrenzanalyse 90

Teil II Eine empirische Untersuchung zur Bedeutung von Kosteninformationen in der Konkurrenzanalyse

6. Kapitel Experimentelle Wirtschaftsforschung anhand von Unternehmensplanspielen 97

7. Kapitel General Management Planspiel MARGA 102

II.7.1 Auswahlkriterien für den experimentellen Planspieleinsatz im Rahmen der vorliegenden Studie. 102

II.7.2 Auswahl des General Management Planspiels MARGA 108

II.7.3 Wettbewerbsumfeld und strategisches Verhalten der Planspiel-Unternehmen bei MARGA und THE BOARD. 117

II.7.4 Konkurrenzanalyse bei MARGA 121

8. Kapitel Versuchsaufbau 130

II.8.1 Anpassung des Instrumentariums und der Versuchsanordnung an die inhaltlichen Erfordernisse der Untersuchung 130

II.8.2 Hypothesen 140

II.8.2.1 Hypothese 1: Strategisches Investitionsverhalten 143

II.8.2.2 Hypothese 2: Produktionsmengenpolitik 145 
II.8.2.3 Hypothese 3: Preispolitik 147

II.8.2.4 Hypothese 4: Forschungs- und Entwicklungspolitik 149

1I.8.2.5 Hypothese 5: Werbestrategie 151

II.8.2.6 Hypothese 6: Wirtschaftlicher Erfolg. 153

9. Kapitel Auswahl der statistischen Testverfahren 155

II.9.1 Wilcoxon-Rangsummen-Test................................... 156

II.9.2 Kolmogorov-Smirnov-Anpassungstest....................... 159

10. Kapitel Ergebnisse der empirischen Untersuchung 162

II.10.1 Hypothese 1: Strategisches Investitionsverhalten 163

II.10.2 Hypothese 2: Produktionsmengenpolitik. 168

II.10.3 Hypothese 3: Preispolitik 171

II.10.4 Hypothese 4: Forschungs- und Entwicklungspolitik ...... 175

II.10.5 Hypothese 5: Werbestrategie.................................. 177

II.10.6 Hypothese 6: Wirtschaftlicher Erfolg .......................... 180

Teil III Die Bedeutung der Untersuchungsergebnisse für die Konkurrenzanalyse 185

11. Kapitel Allgemeingültigkeit der Untersuchungsergebnisse 186

III.11.1 Umfang und Struktur des Teilnehmerfeldes. 186

III.11.2 Werthaltigkeit der Entscheidungsdaten 190

III.11.3 Fokussierung auf Kostenführer 193

III.11.4 Informationsgleichheit 194

III.11.5 Detaillierungsgrad der Information 195

12. Kapitel Interpretation der Untersuchungsergebnisse 197

13. Kapitel Kostenbetrachtung von Wettbewerbern als ein fester Bestandteil der Konkurrenzanalyse 200 
Christoph Heinen - 978-3-631-75315-6

Downloaded from PubFactory at 01/11/2019 06:18:34AM

via free access 


\section{Abbildungsverzeichnis}

Abbildung 1: Der strategische Planungsprozess nach SCHREYöGG............. 13

Abbildung 2: Der strategische Planungsprozess nach WeLGE/AL-LAHAM .... 14

Abbildung 3: Die fünf Wettbewerbskräfte nach PORTER................................. 20

Abbildung 4: Die Konkurrenzanalyse im strategischen Planungsprozess ... 22

Abbildung 5: Der Prozess der strategischen Unternehmensanalyse ........... 23

Abbildung 6: Die Ebenen des Strategiespektrums ...................................... 27

Abbildung 7: Das Strategiekonstrukt nach organisatorischen Ebenen ........ 28

Abbildung 8: Die drei generellen Wettbewerbsstrategien ............................. 29

Abbildung 9: Das Stärken-/Schwächen-Profil nach HAMMER ........................ 43

Abbildung 10: Die Checkliste zur Konkurrenzanalyse nach HOFfMANN ......... 45

Abbildung 11: Ein Fragenkatalog zur Ermittlung von Kostenvorteilen ........... 47

Abbildung 12: Das System zur Konkurrenzanalyse nach PORTER ................. 53

Abbildung 13: Das Reaktionsprofil des Konkurrenten ................................. 55

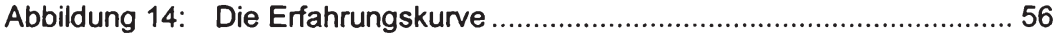

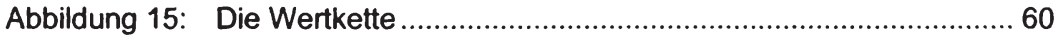

Abbildung 16: Der Wertkettenvergleich in der Konkurrenzanalyse ................61 61

Abbildung 17: Die Portfolio-Analyse von Wettbewerbern ................................. 69

Abbildung 18: Das betriebliche Informationssystem .................................... 73

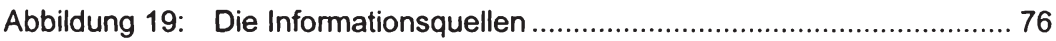

Abbildung 20: Die Informationquellen zur Kostenbetrachtung in der Konkurrenzanalyse 78

Abbildung 21: Der Daten- und Informationsfluss in der Konkurrenzanalyse.. 82

Abbildung 22: Der Entscheidungsprozess zum experimentellen Einsatz eines Unternehmensplanspiels in der vorliegenden Arbeit ... 101

Abbildung 23: Der MARGA-Marktforschungsbericht 123

Abbildung 24: Der MARGA-Jahresbericht 
Abbildung 25: Die Erfahrungskurve in der MARGA-Support Software ........ 126

Abbildung 26: Das BCG-Portfolio in der MARGA-Support Software .......... 127

Abbildung 27: Die Kosteninformation für Teams der Untersuchungsmenge 134

Abbildung 28: Die produktspezifische Deckungsbeitragsrechnung ............ 138

Abbildung 29: Das $\alpha / 2$ - und das $(1-\alpha / 2)$ - Quantil der Standardnormalverteilung ................................................................. 159

Abbildung 30: Die Branchenzugehörigkeit der MARGA-Teilnehmer ........... 188

Abbildung 31: Die Ausbildung der MARGA-Teilnehmer .......................... 188

Abbildung 32: Die Studienrichtung der BOARD-Teilnehmer...................... 189 


\section{Tabellenverzeichnis}

Tabelle 1: Die Klassifizierung von Strategiearten nach KREIKEBAUM .............. 26

Tabelle 2: Die Bestimmung relativer Kosten mit Hilfe der Wertkette ...............63

Tabelle 3: Die Auswahl des Unternehmensplanspiels MARGA ................. 116

Tabelle 4: Die Aufteilung der MARGA- und der BOARD-Teams ................. 131

Tabelle 5: Die Maschinenbelegung der MARGA-Produkte ........................ 136

Tabelle 6: Hypothese 1: Die zugelassenen Datensätze ............................ 144

Tabelle 7: Hypothese 2: Die zugelassenen Datensätze........................... 147

Tabelle 8: Hypothese 3: Die zugelassenen Datensätze ............................ 149

Tabelle 9: Hypothese 4: Die zugelassenen Datensätze ........................... 150

Tabelle 10: Hypothese 5: Die zugelassenen Datensätze ......................... 152

Tabelle 11: Hypothese 6: Die zugelassenen Datensätze .......................... 154

Tabelle 12: MARGA Hypothese 1, Produkt 1, Berechnung der Ränge .......... 164

Tabelle 13: MARGA Hypothese 1, Produkt 1, Teststatistik .......................... 165

Tabelle 14: THE BOARD Hypothese 1, Produkt 1, Berechnung der Ränge .. 166

Tabelle 15: THE BOARD Hypothese 1, Produkt 1, Teststatistik ................... 166

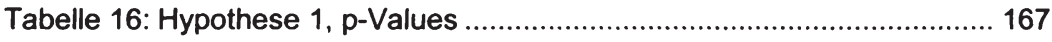

Tabelle 17: MARGA Hypothese 2, Produkt 1, Teststatistik ........................ 169

Tabelle 18: Hypothese 2, p-Values ................................................. 170

Tabelle 19: MARGA Hypothese 3, Produkt 1, Markt 1, Teststatistik ............. 172

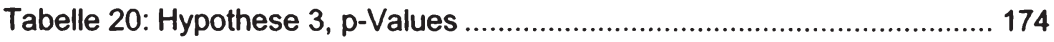

Tabelle 21: MARGA Hypothese 4, Produkt 1, Teststatistik ........................ 176

Tabelle 22: Hypothese 4, p-Values ..................................................... 176

Tabelle 23: MARGA Hypothese 5, Produkt 1, Markt 1, Teststatistik ............. 178

Tabelle 24: Hypothese 5, p-Values ................................................... 179

Tabelle 25: MARGA Hypothese 6, Produkt 1, Berechnung der Ränge ......... 181

Tabelle 26: MARGA Hypothese 6, Produkt 1, Teststatistik 


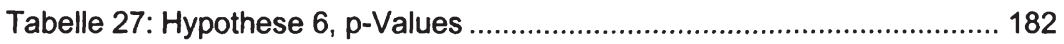

Tabelle 28: Die auswertbaren Antworten der Teilnehmerbefragung .............. 190

Tabelle 29: Die Wahrnehmung der Kosteninformationen............................... 191

Tabelle 30: Die Bedeutung der Kosteninformationen für die strategische

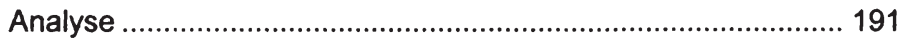

Tabelle 31: Die Bedeutung der Kosteninformationen für Entscheidungen ..... 191

Tabelle 32: Die Beschaffbarkeit von Kosteninformationen .............................. 192

Tabelle 33: Die Informationen über die Ursachen von Kostenvorteilen........... 192 


\section{Abkürzungsverzeichnis}

\begin{tabular}{|c|c|}
\hline Abs. & Absatz \\
\hline Abl. & Ablauf \\
\hline ähnl. & ähnlich \\
\hline allg. & allgemein \\
\hline Aufl. & Auflage \\
\hline a.o. & außerordentlich \\
\hline asymptot. & asymptotisch \\
\hline betriebl. & betrieblich \\
\hline bspw. & beispielsweise \\
\hline bzw. & beziehungsweise \\
\hline ca. & circa \\
\hline d.h. & das heißt \\
\hline ed. & Edition \\
\hline einbeh. & einbehalten \\
\hline et al. & et alii \\
\hline etc. & et cetera \\
\hline evtl. & eventuell \\
\hline f. & folgende Seite \\
\hline F\&E & Forschung und Entwicklung \\
\hline ff. & folgende Seiten \\
\hline filg. & fällig \\
\hline ggf. & gegebenenfalls \\
\hline gem. & gemäß \\
\hline GuV & Gewinn- und Verlustrechnung \\
\hline HGB & Handelsgesetzbuch \\
\hline HWPlan & Handwörterbuch der Planung \\
\hline HBR & Harvard Business Review \\
\hline IAS & International Accounting Standards \\
\hline i.d.R. & in der Regel \\
\hline insb. & insbesondere \\
\hline J. & Jahr \\
\hline Jg. & Jahrgang \\
\hline JoM & Journal of Marketing \\
\hline
\end{tabular}


Lief. u. Leist. Lieferungen und Leistungen

M

Markt

Mio.

Millionen

Naturwiss.

Naturwissenschaften

$\mathrm{Nr}$.

Nummer

0.

oder

öffentl.

öffentlich

o.V.

ohne Verfasser

Prod.

Produkt

S.

Seite

SGE

Strategische Geschäftseinheit

SMJ

Strategic Management Journal

s.o.

siehe oben

sonst.

sonstige

strat.

strategisch

Restlfz.

Restlaufzeit

u.a.

unter anderem

US-GAAP

Generally Accepted Accounting Principles in den USA

u.U.

unter Umständen

vgl.

vergleiche

Vol.

Volume (Band)

Wirtschaftswiss.

Wirtschaftswissenschaften

WiSt.

Wirtschaftswissenschaftliches Studium

Z.B.

zum Beispiel

ZfB

Zeitschrift für Betriebswirtschaft

$\mathrm{ZfbF}$

Zeitschrift für betriebswirtschaftliche Forschung

$\mathrm{ZfO}$

Zeitschrift Führung und Organisation 


\section{Problemstellung, Zielsetzung und Aufbau der Arbeit}

Das heutige wirtschaftliche Umfeld ist in vielen Branchen durch eine hohe und tendenziell eher zunehmende Wettbewerbsintensität gekennzeichnet. Gesättigte Märkte, globaler Wettbewerb und verkürzte Produktlebenszyklen durch rasanten technischen Fortschritt sind nur einige Stichworte, die in diesem Zusammenhang oft genannt werden.

Die Antworten, welche die Unternehmen auf den steigenden Wettbewerbsdruck finden, hängen von der individuellen Situation ab und sind damit vielfältig. Die Möglichkeiten reichen vom aktiven Kostenmanagement, über die strategische Neupositionierung bis hin zu Unternehmenszusammenschlüssen und haben das gleiche Ziel, die Wettbewerbsfähigkeit und damit die langfristige Existenz des jeweiligen Unternehmens im kompetitiven Umfeld zu sichern.

Da es keine pauschalen Ansätze oder Konzepte gibt, Wettbewerbsfähigkeit zu erlangen bzw. zu sichern, ist die Grundvoraussetzung für die Bewältigung dieser Aufgabe eine detaillierte Analyse der vorliegenden Wettbewerbssituation. Diese ist im Allgemeinen Teil eines systematischen Prozesses, in dem Ziele definiert, das Wettbewerbsumfeld analysiert, Entscheidungen abgewogen, gefält und entsprechende Maßnahmen ergriffen und kontrolliert werden. Innerhalb der Analysephase erlangt die Konkurrenzanalyse bei steigender Wettbewerbsintensität eine zunehmende Bedeutung.

Die Inhalte der Konkurrenzanalyse entsprechen den Merkmalen von Wettbewerbsunternehmen, die als Grundlage für bestimmte Entscheidungen relevant sind. Diese zu erfassenden und zu verarbeitenden Konkurrenzinformationen werden in zahlreichen Veröffentlichungen im Einzelnen aufgeführt und erläutert. Dabei lässt sich im Schrifttum eine unterschiedliche Bewertung darüber feststellen, inwiefern Kosten von Wettbewerbsunternehmen in der Konkurrenzanalyse zu berücksichtigen sind: Während einige Autoren Kosten konkurrierender Unternehmen nicht berücksichtigen, erlangen sie bei anderen eine wichtige grundlegende Bedeutung für strategische Entscheidungen. Es ist die Zielsetzung der vorliegenden Arbeit, diese Kontroverse zu beleuchten und Aussagen über die Berücksichtigung von Kosten in der Konkurrenzanalyse zu entwickeln. 
Wenn Kosten von Wettbewerbern erfasst und verarbeitet werden, lassen sich daraus eigene und fremde Stärken bzw. Schwächen auf der Kostenebene ableiten. Stellt sich im Rahmen der vorliegenden Arbeit heraus, dass diese Kenntnisse für strategische Entscheidungen relevant sind und dass durch sie die Entscheidungsqualität positiv beeinflusst werden kann, leitet sich daraus die Forderung ab, Kosten von Konkurrenzunternehmen systematisch zu erfassen und im weiteren Entscheidungsprozess entsprechend zu berücksichtigen.

Zur Bearbeitung dieser Problemstellung bietet sich ein dreiteiliger Aufbau an: Der Teil I bildet das theoretische Fundament der Arbeit und führt darauf aufbauend in den zu untersuchenden Themenkomplex, der Kostenanalyse von Wettbewerbern, ein. Dazu wird im 1. Kapitel der Prozess der strategischen Planung umrissen und die Konkurrenzanalyse darin eingeordnet. Nachdem im 2. Kapitel die Konkurrenzanalyse definiert und von verwandten Ansätzen abgegrenzt wird, stehen im 3. Kapitel die Inhalte und die Instrumente der Konkurrenzanalyse im Mittelpunkt. Der Informationsgewinnung, die im 4. Kapitel dargestellt wird, kommt dabei eine wesentliche und damit kritische Bedeutung zu, was im Besonderen für die Kostenanalyse von Wettbewerbern gilt. Die im Schriftum festzustellende Kontroverse über die Berücksichtigung von Kosten in der Konkurrenzanalyse aufgreifend, fokussiert das 5. Kapitel schließlich die Problemstellung der vorliegenden Arbeit. Zu diesem Zweck wird eine Grundhypothese formuliert, die den Ausgangspunkt für die weiterführende Untersuchung darstellt.

Im Teil II wird eine realitätsbezogene Untersuchung der aufgeworfenen Problemstellung durchgeführt. Dies erfolgt mit Hilfe einer empirischen Studie, der auf der Basis eines Unternehmensplanspiels tatsächliche Entscheidungen von Wirtschaftssubjekten zu Grunde liegen. Die Verwendbarkeit dieses Instrumentariums im Rahmen der experimentellen Wirtschaftsforschung wird im 6. Kapitel thematisiert und für den vorliegenden Fall begründet. Die Auswahl eines für diese Studie passenden Planspiels wird anschließend anhand verschiedener Kriterien im 7. Kapitel getroffen. Sie fällt auf das General Management Planspiel MARGA, das im weiteren Verlauf dieses Kapitels vorgestellt wird. Im Versuchsaufbau sind das einzusetzende Instrumentarium und die zu untersuchenden Inhalte miteinander zu verbinden. Dementsprechend werden im 8. Kapitel einerseits, ausgehend von der Grundhypothese, sechs zu über- 
prüfende Einzelhypothesen aufgestellt. Andererseits erfolgt hier die Anpassung des Intrumentariums und der Versuchsanordnung an die inhaltlichen Erfordernisse der Untersuchung. Die zum Einsatz kommenden mathematisch-statistischen Testverfahren werden im 9. Kapitel erläutert. Deren Anwendung und die Darstellung der Testergebnisse für jede der sechs Hypothesen im 10. Kapitel schließen den Teil II ab.

Die Interpretation und die Bewertung der Untersuchungsergebnisse, sowie die daraus resultierenden Folgerungen für die Konkurrenzanalyse sind die zentralen Elemente im Teil III. Zuvor ist im 11. Kapitel die allgemein gültige Aussagefähigkeit der Untersuchungsergebnisse zu diskutieren, die die Grundlage für eine Übertragbarkeit auf die Unternehmenspraxis darstellt. Im 12. Kapitel werden die Ergebnisse der mathematisch-statistischen Testverfahren interpretiert. Die abschließende Bewertung der im Schriftum festgestellten Kontroverse über die unterschiedliche Berücksichtigung von Kosten in der Konkurrenzanalyse erfolgt im 13. Kapitel. Daraus wird eine entsprechende Forderung über die Erfassung und Verarbeitung kostenbezogener Wettbewerbsinformationen im Rahmen der Konkurrenzanalyse abgeleitet. 
Christoph Heinen - 978-3-631-75315-6

Downloaded from PubFactory at 01/11/2019 06:18:34AM

via free access 


\section{Teil I Die Konkurrenzanalyse als Teil der strate- gischen Planung}

Im Teil I wird die vorliegende Studie theoretisch fundiert. Erst nachdem eine Einordnung der Konkurrenzanalyse in das Themengebiet der strategischen Unternehmensplanung erfolgt ist, kann auf dieser Grundlage die empirische Untersuchung im Teil II ausführlich erläutert werden.

Zu diesem Zweck werden im 1. Kapitel Merkmale, unterschiedliche Ansätze und Prozessabläufe der strategischen Unternehmensplanung dargestellt und die Konkurrenzanalyse in diesen Gesamtzusammenhang eingeordnet. Eine Definition und Abgrenzung der Konkurrenzanalyse zu verwandten Begriffen erfolgt im 2. Kapitel. Die Instrumente, mit deren Hilfe konkurrenzanalytische Betrachtungen durchgeführt werden können, und die Inhalte, die durch die jeweiligen Instrumente erfassbar sind, werden im 3. Kapitel erläutert. Das 4. Kapitel beschäftigt sich mit der Informationsbeschaffung in der Konkurrenzanalyse und geht dabei gesondert auf Informationsquellen ein, die für die Kostenbetrachtung in der Konkurrenzanalyse von Bedeutung sind. Die unterschiedliche Wertigkeit, die der Kostenbetrachtung im Rahmen der Konkurrenzanalyse im Schrifttum zukommt, ist Mittelpunkt des 5. Kapitels. Dieses schließt mit der Formulierung einer Grundhypothese ab, die die zu untersuchende Problematik zusammenfasst und die den Ausgangspunkt für die weiteren Untersuchungen bildet. 


\section{Kapitel Strategischer Planungsprozess und Einordnung der Konkurrenzanalyse}

Das Themengebiet der strategischen Unternehmensplanung, synonym wird auch der Begriff "strategische Planung" verwendet, findet nach wie vor in der Lehre und in der Unternehmenspraxis breite Beachtung. ${ }^{1}$ In der Literatur werden unterschiedliche Begriffe, Ansätze und Prozessabläufe vertreten, von denen die wesentlichen im Folgenden vorgestellt werden.

Im ersten Abschnitt des 1. Kapitels werden unterschiedliche Definitionen der strategischen Unternehmensplanung dargestellt, wobei die Hauptmerkmale zusammenfassend und abschließend aufgezeigt werden. Die Grundzüge des präskriptiv-synoptischen Prozessansatzes der strategischen Planung im Vergleich zum deskriptiv-inkrementalen Ansatz stehen im Mittelpunkt des zweiten Teils. Nachdem der präskriptiv-synoptische Prozessansatz als Grundlage für die wieteren Überlegungen herausgestellt worden ist, werden verschiedene Ablaufund Prozessmodelle der strategischen Planung vorgestellt, die diesem Ansatz zuzurechnen sind. Das Modell von WELGE/AL-LAHAM wird anschließend als Basis für die ausführliche Darstellung des strategischen Planungsprozesses verwendet. Die Konkurrenzanalyse, als wesentlicher Bestandteil dieses Prozesses, wird so in den großen Zusammenhang der strategischen Unternehmensplanung eingebettet.

\section{I.1.1 Definitionen und Merkmale der strategischen Unternehmens- planung}

Vor den ausführlichen Erläuterungen zur strategischen Unternehmensplanung soll an dieser Stelle zunächst der Begriff „Strategie“ definiert und eingeordnet werden.

1 Die Bedeutung des Themengebietes für die Unternehmenspraxis spiegelt sich in den inhaltlichen Schwerpunkten der Personalentwicklung von Unternehmen wider. In einer 1998 von Voss im Rahmen einer wissenschaftlichen Untersuchung durchgeführten branchenübergreifenden Expertenbefragung unter leitenden Personalmanagern in Deutschland konnten inhaltliche Trends im Management Development festgestellt werden. Dabei wird dem Themengebiet ${ }_{n}$ Change Management" unter insgesamt achtzehn inhaltlichen Schwerpunkten der am stärksten steigende Bedarf zugesprochen, gefolgt vom Thema "Strategisches Management". Vgl. VosS, 1999; VoSS/HÄRING/WELGE, 2000, S. $4 \mathrm{ff}$. 
Ausgehend von den militärischen Wurzeln des Strategiebegriffes geben WELGE/ AL-LAHAM einen ausführlichen Überblick über Strategiedefinitionen im deutschen und angloamerikanischen Sprachraum. ${ }^{2}$ Die Vielzahl der unterschiedlichen Sichtweisen berücksichtigend, stellen WELGE/AL-LAHAM folgende Definition auf, die auch dieser Arbeit zu Grunde liegt: Die Strategie beinhaltet „die grundsätzliche, langfristige Verhaltensweise (Maßnahmenkombination) der Unternehmung und relevanter Teilbereiche gegenüber ihrer Umwelt zur Verwirklichung der langfristigen Ziele"3. Dieser enge Strategiebegriff fokussiert demnach die Maßnahmen, die für die Erreichung bestimmter Ziele durchgeführt werden.

Wesentlich breiter als der Strategiebegriff ist die Definition der strategischen Unternehmungsplanung zu sehen. Im Mittelpunkt stehen dabei nach Auffassung des Arbeitskreises "Langfristige Unternehmensplanung" der SCHMALENBACHGESELLSCHAFT die Analyse von Erfolgsquellen und die Entwicklung langfristig angelegter Konzepte zur Zukunftssicherung der Unternehmung. ${ }^{4}$ Es gelte, strukturelle, technische, wirtschaftliche und politische Wandlungen zu erkennen, um Tätigkeitsfelder bzw. Geschäftsfelder, Elemente und Struktur der Unternehmung für künftige Perioden zielorientiert bestimmen zu können.

Für WIESELHUBER bedeutet die strategische Planung "die zukunftsorientierte Weiterentwicklung des Gesamtunternehmens unter Berücksichtigung der planungsrelevanten Umweltfaktoren. Mittels kreativer Leistungen sollen Strategien konzipiert werden, die zum geplanten Wandel und zur Kontinuität des Unternehmens führen." 5

KREIKEBAUM definiert die strategische Unternehmensplanung als einen präskriptiven Prozess, in dem „eine rationale Analyse der gegenwärtigen Situation und der zukünftigen Möglichkeiten und Gefahren zur Formulierung von Absichten, Strategien, Maßnahmen und Zielen führt. Absichten, Strategien, Maßnahmen und Ziele geben an, wie das Unternehmen unter bestmöglicher Aus-

\footnotetext{
2 Vgl. Welge/AL-LAhAM, 1999, S. 12 ff.; Welge/AL-LAhAM, 1992, S. $165 \mathrm{ff}$.

3 WELGE/AL-LAHAM, 1999, S. 19.

$4 \mathrm{Vgl}$. SCHMALENBACHGESELLSCHAFt, 1977, S. $1 \mathrm{ff}$.

5 WIESELHUBER, 1983, S. 56.
} 
nutzung der vorhandenen Ressourcen die durch die Umwelt bedingten Chancen wahrnimmt und die Bedrohungen abwehrt."6

Die drei vorgestellten Definitionen der strategischen Unternehmungsplanung weisen bestimmte Charakteristika auf, die für die weiteren Ausführungen relevant sind:

- das übergeordnete Ziel ist die nachhaltige Sicherung der Überlebensfähigkeit des Gesamtunternehmens,

- der langfristige Zukunftsbezug, der die Planung auf unvollkommenen Informationen basieren lässt,

- die Analyse der planungsrelevanten Umwelt,

- der Gestaltungscharakter, der sich in der Formulierung von Zielen, Strategien und Handlungsalternativen äußert,

- der "Leitfadencharakter", der dem Management und den Mitarbeitern hilft, die täglichen operativen Entscheidungen konform zur strategischen Ausrichtung des Gesamtunternehmens zu fällen,

- die Prozessbezogenheit der strategischen Planung.

\section{I.1.2 Präskriptiv-synoptischer und deskriptiv-inkrementaler Prozess- ansatz der strategischen Planung}

Die oben genannten Charakteristika werden im Prozess der strategischen Planung strukturiert und systematisiert. Das im Rahmen dieser Arbeit gewählte Prozessmodell entspricht dem präskriptiv-synoptischen Prozessansatz. Dieser gibt in normativer Weise Gestaltungsempfehlungen für eine möglichst hohe Effizienz der strategischen Planung. ${ }^{7}$ Der Prozess ist systematisch und entfaltet sich in "geordneten Schrittfolgen"8.

Im Gegensatz dazu steht der deskriptiv-inkrementale Prozessansatz, der bei WELGE/AL-LAHAM zusammenfassend dargestellt ist. ${ }^{9}$ Die in der Unternehmenspraxis beobachteten Abläufe des strategischen Planungsprozesses beschrei-

\footnotetext{
6 KREIKEBAUM, 1997, S. 21.

7 Vgl. WELGE/AL-LAHAM, 1992, S. 35.

8 SCHREYÖGG, 1984, S. 133.

9 Vgl. WELGE/AL-LAHAM, 1992, S. $35 \mathrm{ff}$.
} 
bend, basiert dieser Ansatz auf empirischen Untersuchungen. Das im präskriptiv-synoptischen Ansatz vorgeschriebene phasenweise Durchlaufen des Planungsprozesses wird dabei empirisch nicht belegt. ${ }^{10}$ Vielmehr ist das reale Vorgehen wesentlich unstrukturierter, als es theoretische Modelle vorgeben. SCHREYÖGG ${ }^{11}$ stellt einige Eigenschaften des deskriptiv-inkrementalen Ansatzes vor, die letztlich auf LINDBLOM ${ }^{12}$ zurückgehen:

- Es sollen nur Ziel- und Mittelalternativen geprüft werden, die dem Planer vertraut sind und die auf seinen Erfahrungen, nicht auf Theorien, basieren. Damit "orientiert man sich am Ausgangspunkt des Problemfeldes"13 und lehnt sich am Status Quo an. Eine Beschränkung der Handlungsalternativen nimmt man dabei in Kauf.

- Es soll kein systematischer Gesamtplan eines angestrebten Wunschzustandes, sondern nur kleine Veränderungsschritte entworfen werden. Diese können bei sich ändernden Rahmenbedingungen schneller revidiert werden, wodurch eine größere Flexibilität gewährleistet werden kann.

Auf diesen Ansätzen bauen u.a. MINTZBERG und QUINN auf, die diesen Überlegungen verhaltenswissenschaftliche Elemente hinzufügen. Strategien seien "individuelle Problemlösungen"14, die durch Lernprozesse der betroffenen Mitarbeiter entstehen und wachsen und demnach nicht zentral geplant sind. Nur wenn sie von anderen Mitarbeitern als sinnvolle Problemlösungen anerkannt werden, setzen sie sich durch. Erst in dieser Phase der Strategieentstehung hält MiNTZBERG es für sinnvoll, den Entwicklungsprozess gezielt zu steuern. Für QUINN ist es die Aufgabe des Managements, Vorschläge, Impulse und Strategieformulierungen zu ordnen, zu prüfen und durch eine Zuteilung von Ressourcen zu unterstützen. ${ }^{15}$

Der deskriptiv-inkrementale Prozessansatz der strategischen Planung ist damit im Gegensatz zum präskriptiv-synoptischen Ansatz nicht vollständig planbar, wodurch sich "eine Reihe von Konsequenzen für Inhalte und Methoden der

\footnotetext{
10 Vgl. MintzBerg/Waters, 1990, S. 1 ff.; PetTigrew, 1990, S. 6 ff.

$11 \mathrm{Vgl}$. SCHREYÖGG, 1984, S. $222 \mathrm{f}$.

12 Vgl. LINDBLOM, 1965, S. $130 \mathrm{ff}$.; LINDBLOM, 1969, S. $41 \mathrm{ff}$.

13 WELGE/AL-LAHAM, 1992, S. 37.

14 MintZBerg, 1989, S. 215.

15 Vgl. QUINN, 1988, S. 103.
} 
strategischen Planungslehre ergeben"16 würden. Es sei verfüht, aufgrund einiger Studien eine neue Sichtweise der strategischen Planung zu vertreten, so WeLGe/AL-LAHAM. Außerdem führt SChreyögg kritisch an, dass aus der bloßen Beschreibung erfolgreicher strategischer Veränderungen, die nach dem deskriptiv-inkrementalen Prozessansatz vollzogen wurden, nicht gefolgert werden kann, dass der gleiche Erfolg nicht ebenso gut nach dem präskriptiv-synoptischen Ansatz hätte bewältigt werden können. ${ }^{17}$

SCHREYÖGG sieht in der Einrichtung formalisierter Systeme und Verfahrensweisen zur strategischen Planung, also im präskriptiv-synoptischen Ansatz, diverse Vorteile:18

1. Kontrollierte Entwicklung von Zielen und systematische Auswahl geeigneter Mittel zur Verminderung des Risikos von Fehlentscheidungen,

2. Breitere Analyse aller strategischen Handlungsmöglichkeiten,

3. Frühzeitigere Identifikation strategischer Chancen und Bedrohungen und damit Erhaltung von Handlungsspielräumen,

4. Ausrichtung der operativen und administrativen Einzelentscheidungen an der Gesamtplanung und dadurch Vermeidung widersprüchlicher Einzelschritte,

5. Rationalisierung der Informationsgewinnung, d.h. Berücksichtigung aller relevanten Bereiche, Vermeidung von Doppelarbeit und Vereinheitlichung von Verfahren,

6. Veranlassung des Managements zur kontinuierlichen Zukunftsplanung.

Aufgrund dieser Argumente liegt auch dieser Arbeit das präskriptiv-synoptische Planungsmodell zu Grunde.

\subsubsection{Ablauf- und Prozessmodelle der strategischen Planung}

Im Folgenden werden die Prozessmodelle von GÄLWEILER, WIESELHUBER, SCHREYÖGG und WELGE/AL-LAHAM vorgestellt, die alle dem präskriptiv-synoptischen Ansatz zuzurechnen sind. Aufgrund seiner allgemeinen Gültigkeit wird

16 WELGE/AL-LAHAM, 1992, S. $42 \mathrm{f}$.

17 Vgl. SCHREYÖGG, 1984, S. 243.

$18 \mathrm{Vgl}$. SCHREYÖGG, 1984 , S. 80 f. 
der Ansatz GÄLWEILERS einführend behandelt. Im Gegensatz dazu steht das differenziertere Modell von WIESELHUBER. Der auf diversen Autoren basierende Prozessansatz von SCHREYÖGG rundet die Darstellung unterschiedlicher Modelle im Rahmen dieser Arbeit ab. Grundlage der weiteren Ausführungen wird das Prozessmodell der strategischen Planung von WELGE/AL-LAHAM sein, das für den weiteren Verlauf dieser Untersuchung als das geeignetste Modell anzusehen ist, da es die strategische Analyse sehr detailliert berücksichtigt.

GÄLWEILER versteht unter einem Planungsprozess "das gesamte sachbedingte und zeitbedingte Gefüge einer Menge von Planungsschritten, die zur vollständigen Lösung eines Planungsproblems durchzuarbeiten sind"19. Obwohl die Planungsschritte letztlich von der jeweiligen Aufgabe abhängen, gibt es doch eine allgemein gültige Ablaufstruktur des Planungsprozesses, die mit einer Analyse der Ist-Situation beginnt. GÄLWEILER unterscheidet vier Grundphasen:20

1. Analyse- und Diagnosephase,

2. konkrete Zielformulierung,

3. Strategien-Auswahl und

4. Ausführungsplanung.

Denen geht eine intuitive Vorphase voraus, in der grobe, unstrukturierte Zielund Problemlösungsvorstellungen erdacht werden. Diese Vorphase, die nicht zu den vier Grundphasen gehört, ist weniger durch eine strukturierte Vorgehensweise, als vielmehr durch kreative Vielfalt gekennzeichnet. ${ }^{21}$

Die vier Grundphasen stellen eine Ablaufstruktur dar, die menschlichem Handeln vorausgeht. GäLWEILER beschränkt sich dabei nicht auf wirtschaftliche, sondern bezieht sich auf Planungsprobleme aller Art. Gerade wegen seiner Allgemeingültigkeit ist dieser Ansatz für die vorliegende Studie nicht die geeignete Grundlage. Andererseits wird gerade dadurch ein Rahmen für ein allgemeines Verständnis des Planungsprozesses geschaffen, der den weiteren Ausführungen zu Grunde liegt.

\footnotetext{
19 GÄLWEILER, 1986, S. 185.

$20 \mathrm{VgI}$. GÄLWEILER, 1986, S. $186 \mathrm{ff}$.

21 Vgl. GÄLWEILER, 1986, S. 213.
} 
WIESELHUBER vertritt ein differenzierteres und spezielleres Prozessmodell der strategischen Planung, das auf acht Schritten beruht und - im Gegensatz zu GÄLWEILER - über die reine Planung hinausgeht und zusätzlich die Realisation und die Kontrolle des Geplanten berücksichtigt:22

1. Informationserhebung,

2. Interpretation,

3. Formulierung der Ziele,

4. Entwicklung alternativer Strategien,

5. Bewertung und Auswahl alternativer Strategien,

6. Optimierung der strategischen Pläne,

7. Realisation,

8. Kontrolle.

WIESELHUBER sieht in der strategischen Planung eine Folge von Informationsund Entscheidungsprozessen. Durch die Strukturierung der strategischen Planung reduziert sich der hohe Komplexitätsgrad, wodurch die Transparenz der Planungsaufgabe steigt.

Im Gegensatz zu GÄLWEILER geht WIESELHUBER speziell auf wirtschaftliche Planungsprozesse ein. Sein Ansatz ist für das Grundverständnis der strategischen Unternehmungsplanung in dieser Untersuchung jedoch nicht ausreichend, weil dieser zu wenig detailliert auf den thematischen Schwerpunkt dieser Arbeit, die strategische Analyse, eingeht.

In Anlehnung an verschiedene Autoren ${ }^{23}$ greift SCHREYÖGG bei der Darstellung seines Schemas des strategischen Planungsprozesses auf eine breite Literaturgrundlage zurück. Die Grundzüge mehrerer Modelle werden zusammengefasst und in Abbildung 1 dargestellt:24

SCHREYÖGG sieht zwei "Super-Prozesse“, die das Konzept der strategischen Planung konstituieren, nämlich die Strategieformulierung und die Strategieimplementierung. „Während also der Prozess der Strategieformulierung darauf abstellt herauszufinden, welchen Weg die Unternehmung in Anbetracht ihrer

$22 \mathrm{Vgl.} \mathrm{WIESELHUBER,} \mathrm{1983,} \mathrm{S.} 57 \mathrm{ff}$.

23 Vgl. Steiner, 1969, S. 33; HOFER/SCHENDEL, 1978, S. 47; GLUeCK, 1980, S. 7; STEINER/ MINER, 1982, S. $15 \mathrm{f}$.

24 Vgl. SCHREYÖGG, 1984, S. $84 \mathrm{f}$. 
Ressourcen und zu erwartenden Umweltsituation am besten einschlagen soll, geht es bei der Strategieimplementation darum, die Voraussetzungen für die Erfolgswirksamkeit der gewählten Strategie zu schaffen."25

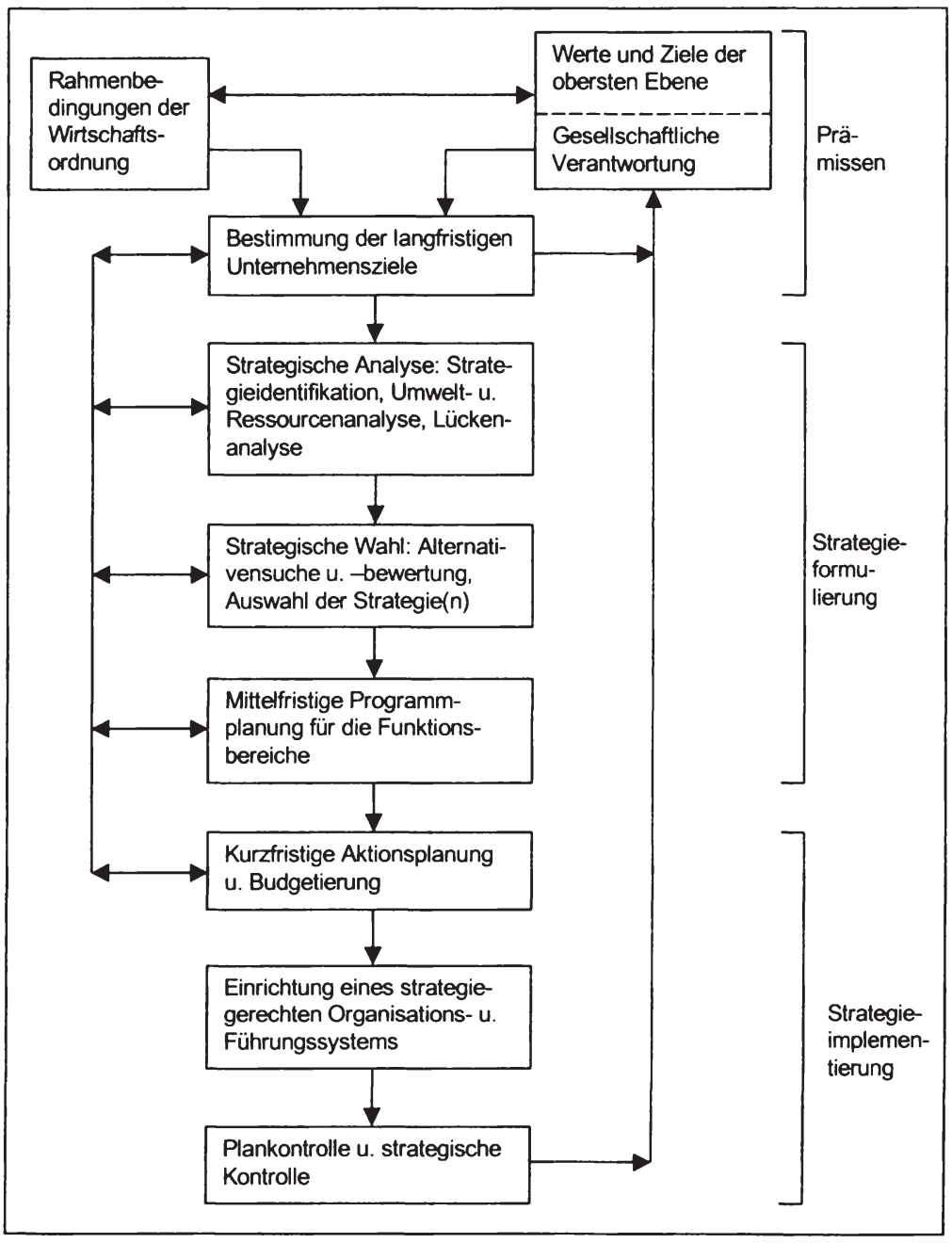

Abbildung 1: Der strategische Planungsprozess nach SCHREYögG ${ }^{26}$

25 SCHREYÖGG, 1984, S. 84.

$26 \mathrm{Vgl}$. SCHREYÖGG, 1984, S. 85. 


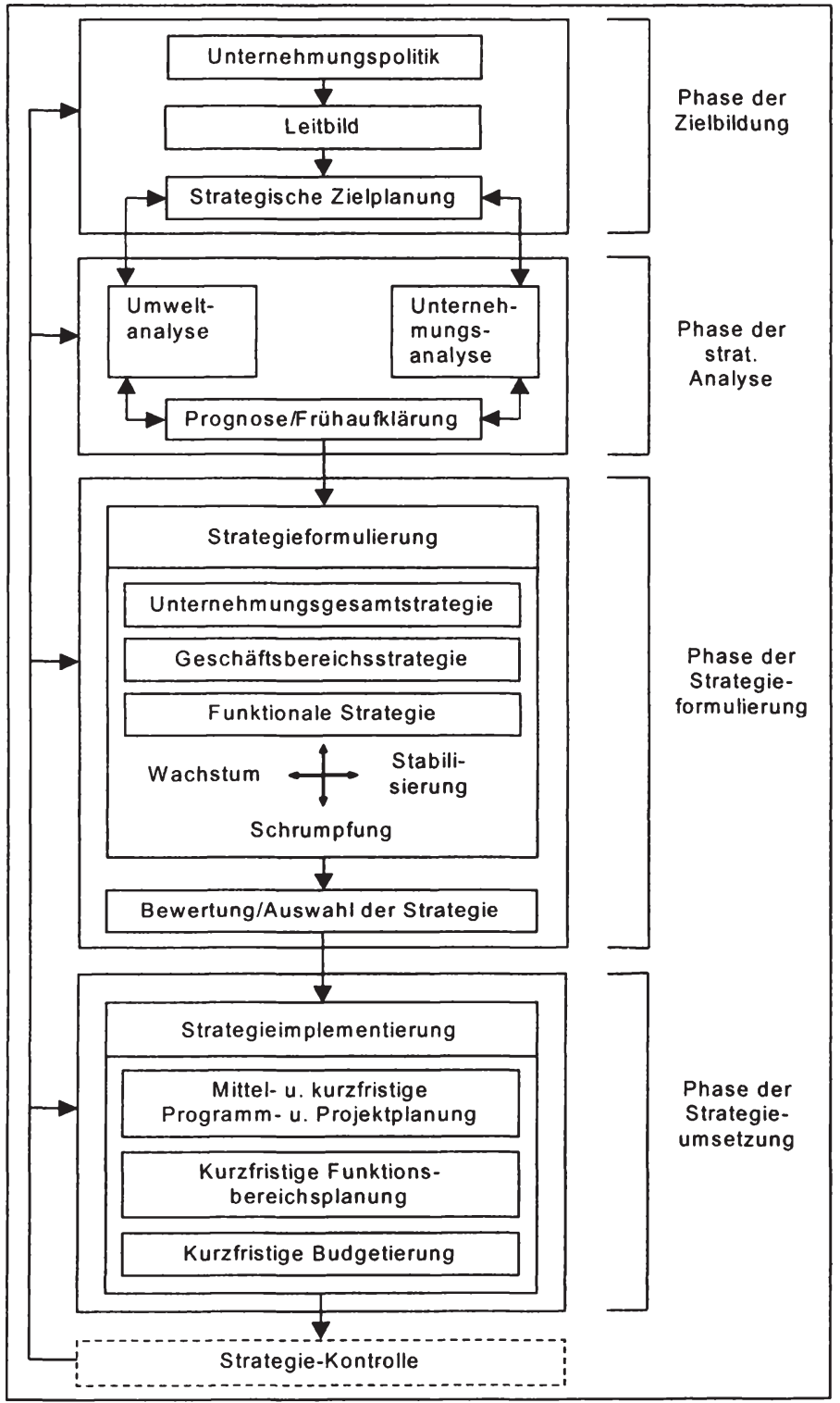

Abbildung 2: Der strategische Planungsprozess nach WELGE/AL-LAHAM ${ }^{27}$

27 Vgl. Welge/Al-LAHAM, 1999, S. 96. 
Die strategische Analyse erhält bei SCHREYÖGG und bei den genannten Autoren, auf die sich SCHREYÖGG bezieht, nur eine untergeordnete Rolle, als Teil des „Super-Prozesses“ Strategieformulierung. Bei WELGE/AL-LAHAM hingegen wird der Strategieformulierung eine eigenständige Phase der strategischen Analyse vorangestellt. Insgesamt gliedern WELGE/AL-LAHAM den strategischen Planungsprozess in die vier Phasen Zielbildung, strategische Analyse, Strategieformulierung und Strategieumsetzung. ${ }^{28}$

Das Ablaufmodell ist in Abbildung 2 dargestellt. ${ }^{29}$

WELGE/AL-LAHAM weisen ausdrücklich darauf hin, dass diese Prozessschritte keine strikte Abfolge von Phasen sind, sondern ein iterativer Prozess, den viele Rückkopplungen und Überlappungen kennzeichnen. Gleiches gilt für das Modell von SCHREYöGG, wie in Abbildung 1 zu sehen ist.

Allen hier vorgestellten Modellen ist die Analysephase im Anfangsstadium des Planungsprozesses gemeinsam, wobei WELGE/AL-LAHAM die strategische Analyse als eigenständige Phase behandeln. Abgeleitet von den Unternehmenszielen wird, differenziert nach Umwelt- und Unternehmensanalyse, eine Prognose bzw. Frühaufklärung durchgeführt, die die Basis für alle weiteren Schritte der Strategieformulierung und -umsetzung ist. Diese Zusammenhänge werden ausführlich bei WELGE/AL-LAHAM erörtert.

Da das Thema dieser Arbeit vorwiegend im analytischen Bereich anzusiedeln ist, wird im Folgenden das Prozessmodel der strategischen Planung nach WELGE/AL-LAHAM zu Grunde gelegt. Zunächst werden die vier Phasen der strategischen Planung erläutert. Die Aspekte, die das Kernthema dieser Studie, die Wettbewerbs- bzw. Konkurrenzanalyse, berühren, werden ausführlich diskutiert. Alle Randthemen dieser Arbeit werden in den großen Kontext eingeordnet, wobei sich die Darstellung auf die wesentlichen Zusammenhänge beschränken wird.

$28 \mathrm{Vgl}$. WELGE/AL-LAHAM, 1999, S. $96 \mathrm{f}$.

${ }^{29} \mathrm{Vgl}$. Welge/AL-LaHAM, 1999, S. 96. 


\section{I.1.3.1 Phase der Zielbildung}

Zur Erreichung von Zielen werden im Planungsprozess Strategien erarbeitet. Noch bevor diese Ziele festgelegt werden, muss jedoch der Zweck der unternehmerischen Tätigkeit definiert sein. Daraus ergeben sich fundamentale Grundsätze des Unternehmens, die die Position tangieren, die das Unternehmen gegenüber unternehmensinternen und -externen Interessengruppen einnimmt und die das spätere Handeln, auf strategischer, wie auf operativer Ebene maßgeblich beeinflusst. Im Leitbild werden u.a. der Unternehmenszweck sowie die allgemein gültigen Unternehmensgrundsätze zusammengefasst, die letztlich die Voraussetzung für eine in sich schlüssige strategische Planung bilden. Erst nachdem diese grundlegenden Zusammenhänge geklärt sind, können Ziele und Strategien erarbeitet werden.

Ein Instrument zur systematischen Ermittlung von Unternehmenszielen ist die Stakeholder Analyse. ${ }^{30}$ Sie wird eingesetzt zur Bestimmung und Bewertung des Anspruchs von unternehmensinternen und -externen Interessengruppen an einem Unternehmen. Die Stakeholder Analyse verdeutlicht die Erwartungen, die verschiedene Interessengruppen an das Unternehmen stellen. Diese Erwartungen, gewichtet mit dem Einfluss der Gruppen, beeinflussen die strategischen Ziele, die im Rahmen der Unternehmensplanung erreicht werden sollen. SCHOLZ schlägt hierfür ein dreistufiges Verfahren vor: ${ }^{31}$

1. die Auflistung möglicher Stakeholder,

2. die Charakterisierung der Interessengruppen nach Ziel- und Machtstrukturen, sowie nach eingegangenem Risiko und

3. die Bestimmung der Relevanz der einzelnen Stakeholder durch ihre Zielhöhe, ihr Machtpotential und durch ihr eingegangenes Risiko.

Die Stakeholder Analyse fordert, Ansprüche relevanter Interessengruppen der Unternehmung bei der Zielformulierung zu berücksichtigen. WELGE/AL-LAHAM stellen weitere Instrumente vor, wie bspw. das PIMS-Modell oder die Kostener-

$30 \mathrm{Vgl}$. WELGE/AL-LAHAM, 1999, S. 165 ff., sowie die dort angegebene Literatur.

$31 \mathrm{Vgl}$. SCHOLZ, 1987, S. $27 \mathrm{ff}$. 
fahrungskurve, die in dieser Phase des Planungsprozesses eingesetzt werden können und die den Stakeholder-Ansatz ergänzen. ${ }^{32}$ Sie fokussieren

1. die empirische Relevanz und

2. die Beziehungen zwischen ausgewählten strategischen Zielen. ${ }^{33}$

Alle drei genannten Instrumente zusammen ermöglichen ein systematisches Vorgehen und gewährleisten die Aufstellung eines in sich schlüssigen Zielsystems. Die Zielformulierung ist ein multipersonaler und kreativer Prozess, auf dessen steuernde Funktion hier nur hingewiesen wird. ${ }^{34}$

\section{I.1.3.2 Phase der strategischen Analyse}

Die Bedeutung der strategischen Analysephase wird in der Literatur sehr hoch eingeschätzt, da hier die Informationen gesammelt und interpretiert werden, die die Grundlage für die Ausarbeitung und Formulierung einer Strategie bilden. ${ }^{35}$ Man unterscheidet zwischen der Umwelt- und der Unternehmensanalyse. ${ }^{36}$

\section{Umweltanalyse}

Bei der Umweltanalyse wird das betriebliche Umfeld eines Unternehmens betrachtet. Auf diese externen Rahmenbedingungen hat das Unternehmen nur einen sehr begrenzten Einfluss. Die Aufgabe der Umweltanalyse ist es, sich bietende Chancen, aber auch zu meidende Risiken, zu identifizieren. Allgemeine Entwicklungen und Trends gilt es frühzeitig in die strategischen Überlegungen einzubeziehen.

\footnotetext{
$32 \mathrm{Vgl}$. WeLGe/AL-LAHAM, 1999, S. $119 \mathrm{ff}$.

33 Das Konzept der Kostenerfahrungskurve wird im 3. Kapitel, Abschnitt 1.3.5, ausführlich dargestellt. Eine detaillierte Darstellung des PIMS Modells findet sich bei: WELGE/AL-LAHAM, 1999, S. $147 \mathrm{ff}$. und bei: BuZELL/GALE, 1989, S. $65 \mathrm{ff}$.

34 Ausführlich: Vgl. WELGE, 1985, S. $164 \mathrm{ff}$.

35 Vgl. GÄLWEILER, 1986, S. 194; KREIKEBAUM, 1997, S. 40.

36 Vgl. Welge/AL-LAHAM, 1999, S. 183.
} 
Erfahrungsgemäß herrscht in der Analysephase ein Informationsüberfluss. Das Herausfinden, Interpretieren und Strukturieren der problemrelevanten Informationen ist daher der wesentliche Tätigkeitsinhalt in dieser Phase. ${ }^{37}$

Zur Strukturierung werden die betrieblichen Umweltbedingungen unterschieden in ein unternehmensspezifisches Wettbewerbsumfeld und in eine globale Umwelt. 38 WELGE/AL-LAHAM verstehen, basierend auf der klassischen Konzeption von FARMER/RICHMANN ${ }^{39}$, unter der globalen Umwelt alle generellen Einflüsse, zu denen das politisch-rechtliche, das sozio-kulturelle, das technologische und das ökonomische Umfeld zählen.

Der Analyse der Wettbewerbsumwelt, im weiteren Sinne dem Kern dieser Arbeit, kommt nach Auffassung PORTERS eine besondere Bedeutung zu. ${ }^{40}$ So besteht die Formulierung einer Wettbewerbsstrategie im Wesentlichen darin, ein Unternehmen in Bezug zu seiner Umwelt zu setzen. Dabei spielen die Kräfte innerhalb einer Branche, in der ein Unternehmen agiert, in der Regel eine wesentlich wichtigere Rolle, als die äußeren Einflüsse.

Unter einer Branche verstehen HAX/MAJLUF eine Gruppe von Unternehmen, die „Produkte oder Dienstleistungen anbieten, welche beinahe Ersatzprodukte füreinander sind"41, d.h. Produkte, die aus Sicht eines Käufers annähernd dieselbe Funktion erfüllen. Die Branchen- und Wettbewerbsanalyse ist ein geordneter Prozess, in dem das relevante Branchenumfeld definiert und das Verhalten der bedeutendsten Wettbewerber bestimmt und charakterisiert wird.

Die Analyse der Wettbewerbsumwelt wird in drei Ebenen eingeteilt, die im Folgenden dargestellt werden:42

1. Analyse der Branchenstruktur,

2. Analyse einer strategischen Gruppe innerhalb einer Branche und

3. Konkurrenzanalyse.

37 Vgl. GÄLWEILER, 1986, S. $218 \mathrm{f}$.

38 Vgl. Welge/AL-LAham, 1999, S. 184; ähnlich: Hilu/Jones, 1989, S. 59 ff.; PEARCE/ Robinson, 1988, S. 99 ff.; THOMAS, 1988, S. 95 ff.

$39 \mathrm{Vgl}$. FARMER/RICHMANN, 1965.

40 Vgl. PORTER, 1999 (a), S. 33.

41 HAX/MAJLUF, 1991, S. 280.

42 Vgl. WELGE/AL-LAHAM, 1992, S. 92. 


\section{ad 1.) Analyse der Branchenstruktur}

Die Analyse der Branchenstruktur nach PORTER bezieht sich auf die Branche als Ganzes. ${ }^{43}$ Der Blickwinkel richtet sich nicht auf einzelne Unternehmen, sondern auf die charakteristischen Eigenschaften und Zusammenhänge, die die Ausprägung des Wettbewerbs einer Branche - und damit die Rentabilität der Einzelunternehmen - bestimmen. Die Wettbewerbssituation innerhalb einer Branche wird auf fünf grundlegende Kräfte zurückgeführt. ${ }^{44}$ Die an PORTER angelehnte Abbildung 3 fasst die wirkenden Kräfte und - stichwortartig - zusätzlich die Determinanten dieser Kräfte zusammen.

HINTERHUBER modifiziert PORTERS Ansatz im Wesentlichen durch Hinzufügen zweier Wettbewerbskräfte, die im kontinentaleuropäischen Wirtschaftsraum traditionell bedeutender sind, als im angelsächsischen Raum: den Eingriff des Staates und die Verhandlungsstärke und das Verhalten der Arbeitnehmer und inrer Organisationen. ${ }^{45}$

ad 2.) Analyse einer strategischen Gruppe innerhalb einer Branche

In der brancheninternen Strukturanalyse werden die einzelnen Unternehmen einer Branche aufgrund ihres strategischen Verhaltens bestimmten Gruppen zugeordnet. Innerhalb dieser strategischen Gruppen verfolgen alle Unternehmen dieselbe oder eine ähnliche Strategie. PORTER klassifiziert das Verhalten von strategischen Gruppen in Dimensionen. Beispiele dafür sind u.a. eine Spezialisierung, die Produktqualität, ein Technologievorsprung oder eine bestimmte Preispolitik. ${ }^{46}$

Auch Unternehmen, die bspw. im Vertrieb oder in der Produktion durch Investitionen in kostensenkende Anlagen und Ausrüstungen einen Kostenvorsprung anstreben, besetzen nach PORTER eine eigene strategische Position, die bei der Einteilung in strategische Gruppen berücksichtigt werden sollte. Bei dieser Zuordnung setzt PORTER implizit voraus, dass die Information über die angestrebte Kostenposition der betreffenden Unternehmen in der Branche bekannt ist.

\footnotetext{
43 Vgl. PORTER, 1999 (a), S. 180.

44 Vgl. PORTER, 1999 (a), S. $33 \mathrm{ff}$.

45 Vgl. HINTERHUBER, 1996 (a), S. 121.

$46 \mathrm{Vgl}$. PORTER, 1999 (a), S. $181 \mathrm{ff}$.
} 


\section{Eintrittsbarrieren \\ Economies of scale \\ Unternehmenseigene \\ Produktionsunterschiede \\ Markenidentität \\ Umstellungskosten \\ Kapitalbedarf \\ Zugang zur Distribution \\ Absolute Kostenvorteile \\ Unternehmensinterne Lernkurve \\ Zugang zu erforderlichen Inputs \\ Unternehmenseigene kosten- \\ günstige Produktgestaltung \\ Staatliche Politik}

Zu erwartende Vergeltungsmaßnahmen
Determinanten der Rivalität

Branchenwachstum

Fix- (oder Lager-) Kosten/Wertschöpfung

Phasen der Überkapazität

Produktunterschiede

Markenidentität

Umstellungskosten

Konzentration u. Gleichgewicht

Komplexe Informationslage

Heterogene Konkurrenten

Strategische Unternehmens-

Neue interessen

Anbieter Austrittsbarrieren

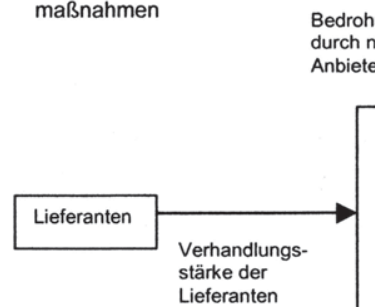

Determinanten der

Lieferantenmacht

Differenzierung der Inputs

Umstellungskosten der

Lieferanten und Unternehmen

der Branche

Ersatz-Inputs

Lieferantenkonzentration

Bedeutung des Auftrags-

volumens für Lieferanten

Kosten im Verhältnis zu den

Gesamtumsätzen der Branche

Einfluß der Inputs auf Kosten

oder Differenzierung

Gefahr der Vorwärtsintegration

im Vergleich zur Gefahr der

Rückwärtsintegration durch

Unternehmen der Branche

Determinanten der

Substitutionsgefahr

Relative Preisleistung der

Ersatzprodukte

Umstellungskosten

Substitutionseignung der

Abnehmer

Determinanten der

Abnehmerstärke

Abnehmerkonzentration gegen

Unternehmenskonzentration

Abnehmervolumen

Umstellungskosten der Abnehmer im Vergleich zu denen des Unternehmens

Informationsstand der Abnehmer

Fähigkeit zur Rückwärtsintegration

Ersatzprodukte

Durchhaltevermögen

Preisempfindlichkeit

Preis/Gesamtumsätze

Produktunterschiede

Markenidentität

Einfluß auf Qualität/Leistung

Abnehmergewinne

Anreize der Entscheidungsträger

Abbildung 3: Die fünf Wettbewerbskräfte nach PORTER ${ }^{47}$

47 Vgl. Welge/AL-LAHAM, 1999, S. 194, in Anlehnung an: PoRTER, 1999 (a), S. 34. 
Für das einzelne Unternehmen wird der Wechsel von einer strategischen Gruppe zu einer anderen durch Eintrittsbarrieren, bzw. durch Mobilitätsbarrieren behindert. Die Stärke der Mobilitätsbarrieren hängt von der angestrebten strategischen Gruppe ab, der sich der Konkurrent anschließen will. ${ }^{48}$ Je stärker Eintritts- und Mobilitätsbarrieren wirken, desto mehr Schutz bieten sie vor branchen- und gruppenfremden Konkurrenten, die in eine strategische Gruppe drängen. Als Hauptursachen für Mobilitätsbarrieren gelten Betriebsgrößenersparnisse, Produktdifferenzierung, Umstellungskosten, Kostenvorteile, Zugang zu Vertriebskanälen, hoher Kapitalbedarf oder staatliche Maßnahmen.

Das Ziel der brancheninternen Strukturanalyse ist die differenzierte Einschätzung der Wettbewerbsintensität und damit des Gewinnpotentials in einer Branche..$^{49}$ Die Höhe der Mobilitätsbarrieren liefert dazu den wichtigsten erklärenden Faktor. Der Grad der Rivalität zwischen unterschiedlichen strategischen Gruppen einer Branche hat erheblichen Einfluss auf die Rentabilität. Je mehr Gruppen sich gebildet haben, je geringer die Unterschiede zwischen diesen Gruppen sind und je niedriger die Mobilitätsbarrieren sind, desto höher ist die zu erwartende Rivalität.

Die brancheninterne Strukturanalyse gibt damit Hinweise auf die strategischen Optionen:50 Schaffung einer neuen strategischen Gruppe, Wechsel in eine günstigere Gruppe, Festigung der bestehenden Position einer Gruppe, z.B. durch Aufbau weiterer Mobilitätsbarrieren, Stärkung der eigenen Position innerhalb der Gruppe, Wechsel in eine neue Gruppe und Stärkung deren struktureller Position.

\section{ad 3.) Konkurrenzanalyse}

In diesem Abschnitt wird die Einordnung der Konkurrenzanalyse in den strategischen Planungsprozess dargestellt. Grundlegende Informationen und das im Rahmen dieser Arbeit verwandte Verständnis der Konkurrenzanalyse findet sich im 2. Kapitel. Die Inhalte und Instrumente werden im 3. Kapitel behandelt.

\footnotetext{
48 Vgl. PORTER, 1999 (a), S. $187 \mathrm{ff}$.

$49 \mathrm{Vgl}$. WELGE/AL-LAHAM, 1992, S. 102.

$50 \mathrm{Vgl}$. PORTER, 1999 (a), S. 208.
} 
Die Konkurrenzanalyse ist Teil der strategischen Analysephase, in der Informationen als Entscheidungsgrundlage im Rahmen der Strategieformulierung gesammelt werden. Neben der Branchenstrukturanalyse und der Analyse der strategischen Gruppen liefert die Konkurrenzanalyse den dritten Blickwinkel auf das Wettbewerbsumfeld eines Unternehmens. Sie stellt damit eine Spezifizierung der Umweltanalyse im Hinblick auf die wichtigsten Konkurrenten des Unternehmens dar. ${ }^{51}$ Abbildung 4 ordnet die Konkurrenzanalyse graphisch in die Struktur des Planungsprozesses ein:

\section{Der Prozess der strategischen Planung}

1. Phase der Zielbildung

2. Phase der strategischen Analyse

3. Phase der Strategieformulierung

4. Phase der Strategieumsetzung

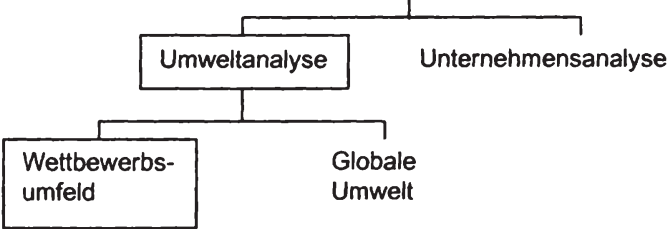

Branchenstrukturanalyse

Analyse strategischer Gruppen

Konkurrenzanalyse

Abbildung 4: Die Konkurrenzanalyse im strategischen Planungsprozess

Das Ziel des externen Analysebereiches ist es, die Chancen und Risiken zu identifizieren, die das Unternehmen von außen beeinflussen. Deren Kenntnis wird aus strategischer Sicht als hoch eingeschätzt. WELGE/AL-LAHAM schließen die Analyse der Wettbewerbsumwelt und der globalen Umwelt mit einem Chancen-/Risiken-Profil ab, das die Ergebnisse in grafischer Form darstellt. 52

51 Vgl. KREIKEBAUM, 1997, S. 121.

$52 \mathrm{Vgl}$. WeLge/AL-LAHAM, 1999, S. $229 \mathrm{f}$. 


\section{Unternehmensanalyse}

Neben der Umweltanalyse, in der Chancen und Risiken des betrieblichen Umfeldes fokussiert werden, steht die Unternehmensanalyse. "Ihr Ziel ist es, ein möglichst objektives Bild der gegenwärtigen und zukünftigen Stärken und Schwächen der Unternehmung aufzuzeigen. "53 Die dafür notwendigen Informationen lassen sich teilweise im eigenen Haus beschaffen. Auf die Schwierigkeiten dabei macht GÄLWEILER aufmerksam. ${ }^{54}$

Das klassische Informationsinstrument ist das betriebliche Rechnungswesen des Unternehmens. Es bietet eine Vielzahl unterschiedlicher Analysemöglichkeiten, die jedoch bei langfristig orientierten strategischen Planungsproblemen, bei denen besonders zukunftsgerichtete, qualitative Daten benötigt werden, nur begrenzt aussagefähig sind, da sie größtenteils vergangenheitsorientiert und nach innen gerichtet sind. .5

Folgenden, in Abbildung 5 dargestellten Prozess der strategischen Unternehmensanalyse schlagen WELGE/AL-LAHAM vor: 56

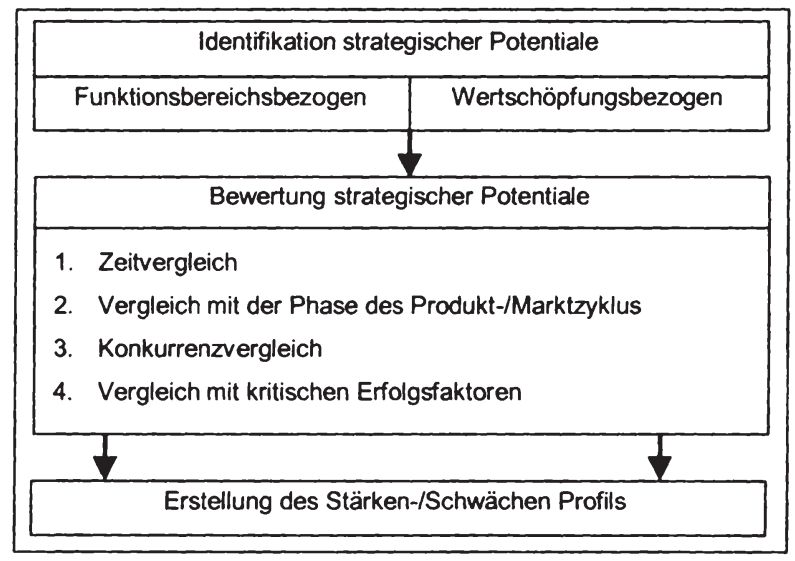

Abbildung 5: Der Prozess der strategischen Unternehmensanalyse ${ }^{57}$

\footnotetext{
53 Welge/AL-LAHAM, 1999, S. 231, in Anlehnung an: HaHN, 1989, S. 2075.

$54 \mathrm{Vgl}$. GÄLWEILER, 1986, S. $202 \mathrm{ff}$.

$55 \mathrm{Vgl.} \mathrm{WELGE/AL-LAHAM,} \mathrm{1999,} \mathrm{S.} 231$.

$56 \mathrm{Vgl}$. WELGE/AL-LAHAM, 1992, S. $109 \mathrm{ff}$.

57 Vgl. WeLge/AL-LAHAM, 1992, S. 110.
} 
Bei der funktionsbereichsbezogenen Identifikation der strategischen Potentiale werden mit Hilfe quantitativer und qualitativer Informationen die Stärken und Schwächen einzelner Unternehmensbereiche gesucht. Die wertschöpfungsbezogene Identifikation der strategischen Potentiale basiert im Wesentlichen auf dem von PORTER entwickelten Konzept der Wertkette. ${ }^{58}$ Ein Unternehmen erlangt nach PORTER einen Wettbewerbsvorteil, indem es die strategisch wichtigen Aktivitäten billiger oder besser als seine Konkurrenten erledigt. Das Konzept der Wertkette dient dazu, strategisch relevante Tätigkeiten zu erkennen, um sie gesondert betrachten, analysieren und bewerten zu können. ${ }^{59}$

Zur Bewertung strategischer Potentiale eines Unternehmens benötigt man Beurteilungsmaßstäbe.60 Während ULRICH drei Kriterien aufzeigt, den Zeitvergleich, den Konkurrenzvergleich und den Soll-Ist-Vergleich, führen WELGE/ALLAHAM vier Möglichkeiten auf, die in Abbildung 5 erwähnt sind. Auf den Konkurrenzvergleich im Rahmen der Unternehmensanalyse, den beide Autoren als Beurteilungsmaßstab für die Ableitung eigener Stärken und Schwächen besonders hervorheben, wird im Folgenden kurz eingegangen.

Stärken oder Schwächen lassen sich oft erst im Vergleich mit Wettbewerbern und anderen Organisationen erkennen. ${ }^{61}$ Der Blick muss daher bei der strategischen Analyse des eigenen Unternehmens nicht nur nach innen, sondern auch nach außen gerichtet sein. KREIKEBAUM sieht die Konkurrenzanalyse daher sogar als eine Voraussetzung der Stärken-/Schwächen-Analyse an ${ }^{62}$, wodurch die Unternehmungsanalyse die Umweltanalyse voraussetzt63. Die wettbewerbsbezogene Relativierung eigener Potentiale bezeichnen WELGE/AL-LAHAM gar als den "wichtigsten Aufgabenbereich der Unternehmungsanalyse"64. Hierbei soll versucht werden, eigene Wertaktivitäten, im Sinne des Wertketten-Konzeptes, mit denen der Wettbewerber zu vergleichen, um so Hinweise zu erhalten, durch welche Schwerpunkte bei bestimmten Wertaktivitäten ein Wettbe-

\footnotetext{
58 Das Wertketten-Konzept und die Möglichkeiten, die die Wertketten-Betrachtung im Rahmen der Konkurrenzanalyse bietet, werden ausführlich im 3. Kapitel, Abschnitt I.3.6, erläutert.

59 Vgl. PORTER, 1999 (b), S. $67 \mathrm{ff}$.

60 VgI. ULRICH, 1990, S. 62.

61 Vgl. GÄLWEILER, 1986, S. 202.

62 Vgl. KREIKEBAUM, 1997, S. 121.

63 Vgl. HAMMER, 1995, S. 40.

64 Welge/AL-Laham, 1992, S. 122.
} 
werbsvorteil erreicht werden kann. Dabei soll auf "grobe Schätzungen“65 zurückgegriffen werden, falls quantitative Informationen über die Wertaktivitäten der Wettbewerber nicht ermittelbar sind.

Wie schon bei der Umweltanalyse können die Ergebnisse der Unternehmensanalyse in einem übersichtlichen Stärken-/Schwächen-Profil dargestellt werden. ${ }^{66}$ Beide großen Analysebereiche gilt es nun zusammenzuführen, um Stärken des Unternehmens mit Chancen zu verbinden, die das externe Umfeld bietet. Diese Gesamtsichtweise wird Chancen-/Gefahrenanalyse genannt. ${ }^{67}$

Die Ergebnisse der Analyse sind das "tragende Fundament"68 aller nachfolgenden Schritte, d.h. die Erkenntnisse, aber auch die Versäumnisse, haben einen direkten Einfluss auf die Folgeschritte und damit letztlich auf die Qualität der Planung. Der Konkurrenzanalyse kommt dabei innerhalb dieser Analysephase eine herausragende Rolle zu.

Wie im Prozessablauf der strategischen Planung deutlich wurde, wird nach der Umwelt- und Unternehmensanalyse die Prognose und strategische Frühaufklärung durchgeführt. Obwohl zukünftig zu erwartende Veränderungen mit in die bisherigen Überlegungen eingeschlossen wurden, wird an dieser Stelle detailliert darauf eingegangen. Der Grund für dieses Vorgehen ist in der maßgebenden Bedeutung zukünftiger Entwicklungen für die strategische Planung zu sehen. Prognosen sind, so WILD, ,... die zweifellos wohl wichtigste Informationsart, die im Zuge der Planung gewonnen und verwendet wird..."69. Dabei liegt nach TÖPFER ein Schwergewicht bei marktorientierten Vorhersagen.70 Hier helfen Verfahren, wie die Extrapolation, Regressionen, die GAP-Analyse oder die Szenario-Technik, Entwicklungen frühzeitig zu erkennen, die bei der langfristig orientierten strategischen Planung von großer Wichtigkeit sind. Auf eine ausführliche Systematisierung der Prognoseinstrumente und der strategischen Frühaufklärungsverfahren wird im Rahmen dieser Arbeit verzichtet. ${ }^{71}$

\footnotetext{
65 WELGE/AL-LAHAM, 1999, S. 275.

$66 \mathrm{Vgl}$. WELGE/AL-LAHAM, 1999, S. $284 \mathrm{ff}$.

67 Vgl. Welge/AL-LAHAM, 1999, S. 284; HAMmer, 1995, S. 42; KREILKAMP, 1987, S. 241.

68 GÄLWEILER, 1986, S. 194.

69 WILD, 1982, S. 87.

$70 \mathrm{Vgl}$. TÖPFER, 1976, S. 189.

71 Vgl. dazu: Welge/AL-LAHAM, 1999, S. $287 \mathrm{ff}$.
} 


\section{I.1.3.3 Phase der Strategieformulierung}

Die Phase der Strategieformulierung bezeichnen WELGE/AL-LAHAM als den „Kernbereich des strategischen Managementprozesses"72. An dieser Stelle werden Strategien - im Sinne von Maßnahmen - erarbeitet, die zur Erreichung bestimmter Ziele in Frage kommen.

Dazu leiten WELGE/AL-LAHAM aus Vorüberlegungen anderer Autoren ${ }^{73}$ folgende Prinzipien als Grundlage für die Strategieformulierung ab:74 Aufbau von Stärken, Vermeidung von Schwächen, Konzentration der Kräfte, Optimierung der Ressourcenbasis und Ausnutzung bzw. Aufbau von Synergiepotentialen.

\begin{tabular}{|l|l|}
\hline $\begin{array}{l}\text { Unterscheidungskri- } \\
\text { terium/Gegenstand }\end{array}$ & Bezeichnung \\
\hline $\begin{array}{l}\text { Organisatorischer } \\
\text { Geltungsbereich }\end{array}$ & $\begin{array}{l}\text { Unternehmensgesamtstrategien } \\
\text { Geschäftsbereichsstrategien } \\
\text { Funktionsbereichsstrategien }\end{array}$ \\
\hline Funktionsbereich & $\begin{array}{l}\text { Absatzstrategien } \\
\text { Produktionsstrategien } \\
\text { F\&E - Strategien } \\
\text { Investitionsstrategien } \\
\text { Finanzierungsstrategien } \\
\text { Personalstrategien }\end{array}$ \\
\hline $\begin{array}{l}\text { Entwicklungsrichtung//2n } \\
\text { Mitteleinsatz }\end{array}$ & $\begin{array}{l}\text { Wachstumsstrategien (Investieren) } \\
\text { Stabilisierungsstrategien (Halten) } \\
\text { Schrumpfungsstrategien (Desinvestieren) }\end{array}$ \\
\hline Marktverhalten & $\begin{array}{l}\text { Angriffsstrategien } \\
\text { Verteidigungsstrategien }\end{array}$ \\
\hline Produkte/Märkte & $\begin{array}{l}\text { Marktdurchdringungsstrategien } \\
\text { Marktentwicklungsstrategien } \\
\text { Produktentwicklungsstrategien } \\
\text { Diversifikationsstrategien }\end{array}$ \\
\hline $\begin{array}{l}\text { Wettbewerbsvorteile/M } \\
\text { arktabdeckung }\end{array}$ & $\begin{array}{l}\text { Strategien der Kostenführerschaft } \\
\text { Differenzierungsstrategien } \\
\text { Konzentrationsstrategien (auf Kostenführer- } \\
\text { schaft oder Produktdifferenzierung) }\end{array}$ \\
\hline
\end{tabular}

Tabelle 1: Die Klassifizierung von Strategiearten nach KREIKEBAUM ${ }^{75}$

72 WELGE/AL-LAHAM, 1999, S. 311.

73 Vgl. PÜMPIN, 1980, S. 15 ff.; HINTERHUBer, 1990, S. 108; HeNtZE et al., 1993, S. 144 ff.; HAMMER, 1991, S. $116 \mathrm{f}$.

74 Vgl. Welge/AL-LAHAM, 1999, S. 311.

$75 \mathrm{Vgl}$. KREIKEBAUM, 1997, S. 58. 
KREIKEBAUM führt unterschiedliche Strategiearten auf, deren Klassifizierung in Tabelle 1 dargestellt ist.

In Anlehnung an ANSOFF verbindet KREIKEBAUM unterschiedliche Strategiearten miteinander, indem bspw. eine Produkt-/Markt-Strategie mit einer funktionalen Strategie wie einer bestimmten F\&E-Strategie kombiniert wird. So kann eine Diversifikation die Entwicklung neuer Produkte und Verfahren erfordern. ${ }^{76}$

WELGE/AL-LAHAM differenzieren das Strategiespektrum hingegen nach der geplanten Entwicklungsrichtung des Leistungsprogrammes der Unternehmung und nach dem organisatorischen Geltungsbereich einer Strategie. ${ }^{77}$ Abbildung 6 spiegelt diese Aufteilung wider:

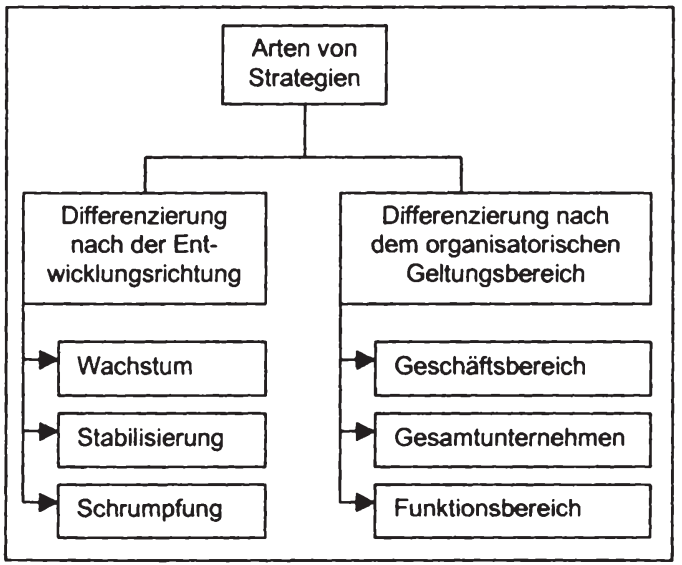

Abbildung 6: Die Ebenen des Strategiespektrums ${ }^{78}$

Die Differenzierung der Strategien nach organisatorischen Geltungsbereichen ist abhängig von der Komplexität der Organisationsstruktur des Unternehmens. Dabei bringen unterschiedliche Organisationsebenen auch unterschiedliche Planungs- und Strategieebenen mit sich, die aufeinander abgestimmt und miteinander verwoben sind. Abbildung 7 stellt die Differenzierung des Strategiekonstruktes nach organisatorischen Ebenen dar:

${ }^{76} \mathrm{Vgl}$. ANSOFF, 1957, S. 114.

$77 \mathrm{Vgl}$. WELGE/AL-LAHAM, 1999, S. $320 \mathrm{ff}$.

78 Vgl. Welge/AL-LAHAM, 1999, S. 321. 


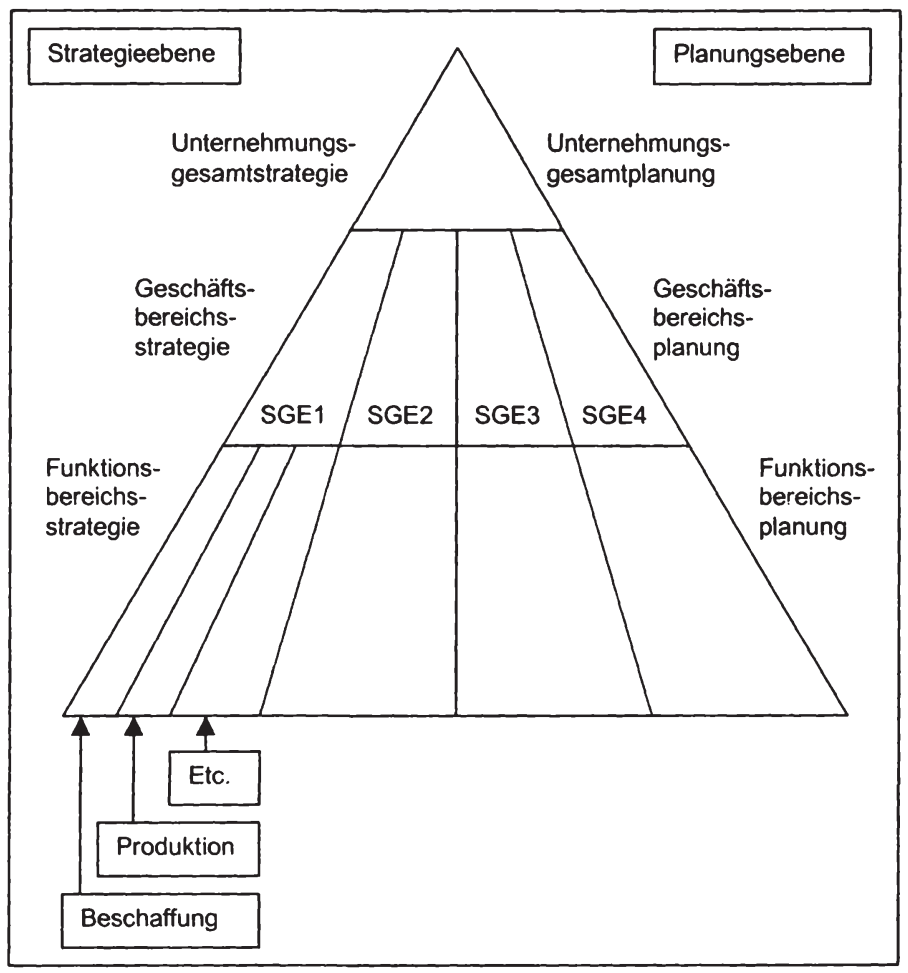

Abbildung 7: Das Strategiekonstrukt nach organisatorischen Ebenen ${ }^{79}$

Bei der Planung der drei Ebenen, Gesamtunternehmen, Geschäftsbereich und Funktionsbereich, kommen unterschiedliche Instrumente zum Einsatz. Auf Gesamtunternehmensebene gilt es, eine Strategie für die Gesamtheit der unterschiedlichen Geschäftseinheiten zu erarbeiten. Auf der Suche nach einem ausbalancierten Verhältnis zwischen wachsenden, schrumpfenden und gewinnbringenden Geschäftsbereichen ${ }^{80}$ werden Portfolio-Analysen eingesetzt, auf die im 3. Kapitel, Abschnitt I.3.7, noch ausführlich eingegangen wird.

Auf Geschäftsbereichsebene stehen marktnahe Strategien im Vordergrund. PORTER sieht drei generelle Wettbewerbsstrategien von Geschäftsbereichen:

79 Vgl. WELGE/AL-LAHAM, 1999, S. 323.

$80 \mathrm{Vgl}$. WELGe/AL-LAHAM, 1999, S. $322 \mathrm{ff}$. 


\begin{tabular}{|c|c|c|}
\hline $\begin{array}{c}\text { Strategischer } \\
\text { Vorteil } \\
\text { Strategisches } \\
\text { Zielobjekt }\end{array}$ & $\begin{array}{c}\text { Singularität aus der } \\
\text { Sicht des Käufers }\end{array}$ & Kostenvorsprung \\
\hline Branchenweit & Differenzierung & $\begin{array}{c}\text { Umfassende } \\
\text { Kostenführerschaft }\end{array}$ \\
\hline \begin{tabular}{|c|} 
Beschränkung \\
auf ein Segment
\end{tabular} & Konzentration auf Schwerpunkte \\
\hline
\end{tabular}

Abbildung 8: Die drei generellen Wettbewerbsstrategien ${ }^{81}$

Die Strategie der Differenzierung zeichnet sich durch Einzigartigkeit und Unnachahmbarkeit des Produktes bzw. der Leistung aus. ${ }^{82}$ „Die strategische Grundkonzeption der Differenzierung besteht darin, in einem Marktsegment durch Produkt- und/oder Verfahrensinnovationen sowie durch Standortanpassungen eine monopolähnliche Stellung einzunehmen."83

Beim Strategietyp der Konzentration auf Schwerpunkte werden Marktnischen fokussiert, auf die das gesamte Unternehmen ausgerichtet wird. Bei diesen Marktnischen handelt es sich bspw. um bestimmte Abnehmergruppen, bestimmte Teile des Produktionsprogramms oder um einen geographisch abgegrenzten Markt. ${ }^{84}$

Der Strategie der Kostenführerschaft kommt im Rahmen dieser Arbeit eine wesentliche Bedeutung zu, weil die Operationalisierung dieses Strategietyps u.a. in die Formulierung der Hypothesen einfließt. Die Kostenführerstrategie wird an dieser Stelle im Rahmen der Strategieformulierung behandelt, um die Position im Gesamtprozess der strategischen Unternehmensplanung zu verdeutlichen. Sie wird im Folgenden ausführlicher dargestellt.

\footnotetext{
$81 \mathrm{Vgl.} \mathrm{PORTER,} 1999$ (a), S. 75.

82 Vgl. PORTER, 1999 (a), S. 73 f.

83 HINTERHUBER, 1996 (a), S. 180.

$84 \mathrm{Vgl}$. PORTER, 1999 (a), $75 \mathrm{ff}$.
} 
Die Minimierung der Kosten ist der Leitfaden der Kostenführerschaft. Einer Strategiewahl in dieser Richtung geht eine Kostenanalyse voraus ${ }^{85}$, bei der nicht nur die Kosten des eigenen Unternehmens zu betrachten sind, sondern bei der die eigene Kostensituation mit der der wichtigsten Konkurrenten verglichen wird.

PORTER schlägt dafür das Konzept der Wertkette vor. Durch eine detaillierte Betrachtungsweise, die durch die Aufteilung des Gesamtunternehmens in einzelne Aktivitäten erreicht wird, lassen sich strategische Schwerpunktaktivitäten, aber auch Kosten von Wettbewerbern abschätzen. ${ }^{86}$

Die Strategie der Kostenführerschaft ist eng verbunden mit dem Ansatz der Kostenerfahrungskurve. Dieser belegt empirisch den kostensenkenden Effekt einer Massenstrategie, wobei hohe kumulierte Produktionszahlen zu sinkenden Kosten führen, was für eine erfolgreiche Kostenführerschaft eine notwendige Voraussetzung ist. ${ }^{87}$

Gemäß dem Konzept der Erfahrungskurve begünstigt ein hoher Marktanteil die Entwicklung eines Kostenvorsprungs gegenüber Wettbewerbern. ${ }^{88}$ Eine damit einhergehende Ausweitung der Produktionsmenge, die eine Betriebsvergrößerung, bspw. einen Kapazitätsaufbau ${ }^{89}$ voraussetzen kann, führt oft zu einer Fixkostendegression, die letztlich die Stückkosten weiter senkt. Hinzu kommen bei einem hohen Marktanteile oft weitere Einsparmöglichkeiten, die sich Z.B. im Einkauf eröffnen. Mit der expansiven Produktionspolitik geht ein aggressives Marketing einher, das es ermöglichen soll, die erhöhten Mengen abzusetzen. Insofern ist die Entscheidung für eine Strategie der Kostenführerschaft mit diversen Folgeentscheidungen verbunden, durch die diese erst realisiert bzw. langfristig gehalten werden kann.

\footnotetext{
$85 \mathrm{Vgl}$. WeLGE/AL-LAHAM, 1999, S. 377.

86 Auf das Wertketten-Konzept und auf die Möglichkeiten, die die Wertketten-Betrachtung im Rahmen der Konkurrenzanalyse eröffnet, wird ausführlich im 3. Kapitel, Abschnitt I.3.6, eingegangen.

87 Das Konzept der Erfahrungskurve wird detailliert im 3. Kapitel, Abschnitt 1.3.5, dargestellt. $88 \mathrm{Vgl}$. PORTER, 1999 (a), S. 72.

89 Vgl. PORTER, 1999 (a), S. 71.
} 
Eine Kostenführung stärkt die eigene Position gegenüber Lieferanten aufgrund eines größeren Spielraumes bezüglich der Input-Preise. Gegenüber mächtigen Abnehmern ist man gegen stark sinkende Verkaufspreise geschützt, da man sich immer in einer günstigeren Position als die Konkurrenten befindet. Dies gilt ebenso für die Rivalität innerhalb der Branche. Niedrige Kosten erschweren potentiellen Konkurrenten den Markteintritt und Ersatzprodukten, sich zu etablieren. Damit verbessert die Kostenführerstrategie die eigene Position gegenüber allen fünf Wettbewerbskräften, die bereits erläutert wurden. ${ }^{90}$

STEIN weist darauf hin, dass als Voraussetzung für eine Kostenführerstrategie der Preis auf dem betreffenden Markt ein relevantes Wettbewerbsinstrument sein muss. ${ }^{91}$ Eine günstige Kostenposition eröffnet damit Spielräume bei der Preisgestaltung, die auf preissensiblen Märkten von größerer Bedeutung sind als auf preisunsensiblen Märkten.

Folgende Risiken und Nachteile einer Kostenführerstrategie zählen WELGE/AL-

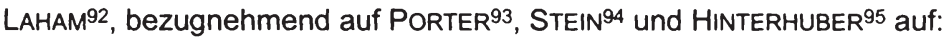

- mangelnde Flexibilität und Anpassungsfähigkeit an veränderte Marktverhältnisse,

- Gefährdung des Kostenvorsprungs durch raschen technologischen Fortschritt und

- tendenzielle Innovationsfeindlichkeit.

Trotz dieser Risiken ist die Kostenführung für viele Unternehmen eine Strategie, bei der die oben genannten Vorteile überwiegen, zumal viele Unternehmen im heutigen globalisierten Wirtschaftssystem zum Erreichen einer günstigen Kostenposition gezwungen sind.

Die drei vorgestellten Strategietypen, Differenzierung, Konzentration auf Schwerpunkte und die Kostenführerschaft, sind - im Bereich der Strategieformulierung - den Geschäftsbereichsstrategien zuzuordnen. Im Sinne der

\footnotetext{
$90 \mathrm{Vgl.} \mathrm{PORTER,} 1999$ (a), S. $71 \mathrm{f}$.

$91 \mathrm{Vgl.STEIN}, 1988$, S. $404 \mathrm{f}$.

$92 \mathrm{Vgl}$. WELGE/AL-LAHAM, 1999, S. $381 \mathrm{f}$.

93 Vgl. PORTER, 1999 (a), S. 83 f.

$94 \mathrm{Vgl}$. STEIN, 1988, S. $406 \mathrm{ff}$.

$95 \mathrm{Vgl}$. HINTERHUBER, 1990, S. $98 \mathrm{f}$.
} 
Strategiedifferenzierung nach den organisatorischen Ebenen, Gesamtunternehmen, Geschäftsbereiche und Funktionalbereiche ${ }^{96}$, geben die funktionalen Strategien die Konsequenzen für die Funktionsbereiche wider und stellen die konkrete Interpretation der Gesamtunternehmens- und der Geschäftsbereichsstrategien dar. Sie fungieren als Schnittstelle zwischen Strategie und operativer Umsetzung, wobei innen dabei eine Koordinations- und Harmonisierungsfunktion zukommt. ${ }^{97}$

Damit ist die Darstellung der Strategiearten, differenziert nach organisatorischen Geltungsbereichen, abgeschlossen. Strategiearten lassen sich darüber hinaus nach der Entwicklungsrichtung Wachstum oder Schrumpfung differenzieren, worauf jedoch im Rahmen dieser Arbeit nicht weiter eingegangen werden soll. $98 \mathrm{Im}$ letzten Schritt vor der Umsetzung wird die erarbeitete Strategie auf bestimmte Kriterien hin untersucht und bewertet. Hier fließen alle vorhandenen Informationen zusammen, bevor man sich für die Umsetzung einer strategischen Alternative entscheidet. ${ }^{99}$

\subsubsection{Phase der Strategieumsetzung}

In der Phase der Strategieimplementierung werden die strategischen Pläne in konkretes, strategiegeleitetes Handeln der Unternehmungsmitglieder umgesetzt. ${ }^{100}$ KoLKS definiert die Strategieimplementierung als sachorientierte Umsetzung und verhaltensorientierte Durchsetzung strategischer Maßnahmenprogramme. ${ }^{101}$

An dieser Stelle erfolgt die Verzahnung der strategischen mit der operativen Planung, die durch eine kurzfristige, bereichs- oder abteilungsbezogene Maßnahmen-, Termin- und Budgetplanung gekennzeichnet ist. ${ }^{102}$

\footnotetext{
$96 \mathrm{Vgl}$. Abbildung 7.

97 Vgl. Welge/AL-LAHAM, 1999, S. 401, in Anlehnung an: Hinterhuber, 1996 (b), S. 5 ff.; Hentze et al., 1993, S. 160 f.; PÜMPIN, 1980, S. 50 f.

$98 \mathrm{Zu}$ diesem Thema weiterführend: vgl. WELGE/AL-LAHAM, 1999, S. $432 \mathrm{ff}$.

$99 \mathrm{Vgl}$. WELGE/AL-LAHAM, 1999, S. $484 \mathrm{ff}$.

100 Vgl. NUTT, 1989, S. 145; JOHNSON/SCHOLES, 1988, S. 15.

101 Vgl. KOLKS, 1990, S. 79.

102 Vgl. WeLge/AL-LAHAM, 1999, S. 526.
} 
Die operative Planung im Rahmen der Strategieimplementierung unterteilt sich in folgende Bereiche: 103

1. Mittelfristige Programm- und Projektplanung

2. Operative Funktionsbereichsplanung

- Operative Beschaffungsplanung

- Operative Produktionsplanung

- Operative Planung der Forschung und Entwicklung

- Operative Marketingplanung

3. Budgetierung

Alle operativen Maßnahmen zur Umsetzung einer Strategie sind eng aufeinander abzustimmen und zu koordinieren. Auf die unterschiedlichen Bereiche der Strategieimplementierung soll im Rahmen dieser Untersuchung nicht näher eingegangen werden. 104

Damit ist die Darstellung der vier Phasen des strategischen Planungsprozesses, Zielbildung, strategische Analyse, Strategieformulierung und Strategieimplementierung, abgeschlossen. Die Konkurrenzanalyse wurde dabei als ein fundamentaler Bestandteil des strategischen Planungsprozesses hervorgehoben, da sie wesentlicher Teil der Analysephase ist, die wiederum die Basis für die eigentliche Strategieformulierung bildet.

103 Vgl. WeLGe/AL-LAHAM, 1999, S. $547 \mathrm{ff}$.

104 Zu diesem Thema weiterführend: vgl. WELGE/ALLLAHAM, 1999, S. $523 \mathrm{ff}$. 


\section{Kapitel Definition und Abgrenzung der Konkurrenzanalyse}

Nach der Einordnung in den Gesamtzusammenhang des strategischen Planungsprozesses werden im 2. Kapitel die Konkurrenzanalyse sowie einige grundlegende Begriffe definiert und im Weiteren von verwandten Ansätzen abgegrenzt.

\subsubsection{Definition der Konkurrenzanalyse}

Konkurrenz im ökonomischen Sinne ist der gegenseitige Wettbewerb zwischen Anbietern oder zwischen Nachfragern auf einem Markt. Nachfragewettbewerb entsteht, weil Interessenten gleiche Güter- oder Dienstleistungskombinationen verlangen, deren Angebot begrenzt ist. Wettbewerb zwischen Anbietern kommt dadurch zustande, dass mehrere Konkurrenzunternehmen nach dem gleichen Ziel streben, nämlich nach dem Absatz von Produkten oder Leistungen, die auf den selben Bedarf abzielen. ${ }^{105}$ Wettbewerb bezieht sich ausschließlich auf Vorgänge, die sich auf einer Marktseite, d.h. auf der Angebots- oder der Nachfrageseite abspielen, während ein Markt die Interaktion zwischen beiden Marktseiten umfasst. ${ }^{106}$

HOFFMANN unterscheidet drei Konkurrenzklassen:107

\section{Ressourcen Konkurrenz:}

Ressourcen, die nicht in beliebiger Menge verfügbar sind, werden von mehreren Unternehmen benötigt, was oft zu Verteilungskämpfen, bzw. zu höheren Preisen für diese Ressourcen führt.

\section{Vertikale Konkurrenz:}

Jedes Unternehmen ist in einer bestimmten Weise im Wertschöpfungsprozess vom Rohstoff bis zum fertigen Produkt positioniert. Es bezieht Produkte oder Leistungen von der vorgelagerten Lieferantenseite und gibt Produkte oder Leistungen an nachgelagerte Stufen ab. Hier kann es zum Ver-

105 Vgl. ILLIG, 1980, S. 5, in Anlehnung an: RöPER, 1952, S. 11; GEIST, 1954, S. 5 f.; SCHNEIDER, 1985, S. $497 \mathrm{f}$.

106 Vgl. SCHNEIDER, 1985, S. 470.

107 Vgl. HOFFMANN, 1986, S. $186 \mathrm{ff}$. 
teilungswettbewerb um Aktivitäten innerhalb des Wertschöpfungsprozesses kommen, denn aufgrund der Aktivitäten werden letztlich die im Gesamtprozess zur Verfügung stehenden finanziellen Mittel verteilt.

\section{Horizontale Konkurrenz:}

Unternehmen der gleichen Wertschöpfungsebene stehen bezüglich ihrer gleichen Abnehmer in Konkurrenz zueinander. Die horizontale Konkurrenz bezieht sich damit nicht nur auf gesamte Unternehmen, sondern auch auf einzelne Funktionsbereiche eines Unternehmens, die mit externen Anbietern konkurrieren.

Im Rahmen dieser Arbeit steht der Wettbewerb zwischen Unternehmen auf der Angebotsseite im Mittelpunkt der Betrachtung, die um eine begrenzte Nachfrage konkurrieren.

Als Konkurrenzanalyse, synonym wird auch der Begriff „Konkurrentenanalyse“ verwendet, wird „die Analyse aller Daten der Konkurrenzunternehmen bezeichnet, die für eigene Entscheidungen im Rahmen der strategischen Unternehmensplanung von Bedeutung sind“108.

LINK unterscheidet in Anlehnung an SAMMON und FISCHER ${ }^{109}$ zwischen einer strategischen und einer operativen Konkurrenzanalyse. ${ }^{110}$ Während man die strategische Konkurrenzanalyse zur Erstellung einer Wettbewerbsstrategie benötigt, wird unter der operativen Konkurrenzanalyse die Beschaffung aller wettbewerbsrelevanten Informationen verstanden, die bei der Umsetzung einer Strategie benötigt werden.

Im Mittelpunkt der Betrachtung stehen jeweils die stärksten Konkurrenten, wobei ausdrücklich davor gewarnt wird, kleine aber flexible und wachstumsstarke Unternehmen in der Konkurrenzanalyse zu vernachlässigen. ${ }^{111}$ HAX/MAJLUF sprechen daher von den relevantesten Konkurrenten. ${ }^{112}$ Ein relevanter Konkurrent ist ein Mitbewerber, der eine oder mehrere der folgenden Bedingungen erfüllt:

\footnotetext{
108 KREIKEBAUM, 1997, S. 121.

109 Vgl. SAMMON, 1985, S. $111 \mathrm{ff.;} \mathrm{FISCHER,} \mathrm{1986,} \mathrm{S.} 103$.

110 Vgl. LINK, 1988, S. $34 \mathrm{ff}$.

111 Vgl. GÄlWEILER, 1986, S. 370; HOFFMANN, 1986, S. 191; KREIKEBAUM, 1997, S. 122.

$112 \mathrm{Vgl}$. HAX/MAJLUF, 1991, S. $338 \mathrm{f}$.
} 
- Hinsichtlich des Marktes

- besitzt er einen hohen Marktanteil;

- hat er ein fundiertes Marktwachstum erlebt;

- erwirtschaftet er gemessen am Branchendurchschnitt hohe Gewinnspannen;

- $\quad$ hat er eine aggressive Wettbewerbshaltung gezeigt;

- ist er für die Wettbewerbsmaßnahmen, die das eigene Unternehmen zeigt, überaus verletzlich.

- Hinsichtlich der Funktionen

- hat er die günstigste Kostenstruktur;

- hat er die modernste technische Basis;

- hat er das aggressivste Marketing;

- bietet er die höchste Produktqualität;

- verfügt er über das Höchstmaß an vertikaler Integration;

- verfügt er über den höchsten Grad an Kapazitätsausnutzung.

Neben den bedeutenden existierenden Wettbewerbern fordert PORTER die Analyse potentieller Wettbewerber. ${ }^{113}$ Hierzu zählt er:

- Unternehmen außerhalb der Branche, die Eintrittsbarrieren leicht überspringen können,

- Unternehmen, für die der Eintritt in die Branche offensichtlich von Interesse sein würde,

- Unternehmen, für die die Teilnahme am Wettbewerb in der Branche eine Erweiterung ihrer Unternehmensstrategie darstellt und

- Abnehmer oder Zulieferer, die vielleicht rückwärts oder vorwärts integrieren wollen.

Darüber hinaus empfiehlt PORTER, sich mit möglichen zukünftigen Fusionen und Beteiligungen zu beschäftigen, da auf diese Weise bisher schwache Wettbewerber plötzlich deutlich an Stärke zunehmen können.

Das Ziel der Konkurrenzanalyse besteht nach PORTER in der Herausarbeitung

113 Vgl. PORTER, 1999 (a), S. 89. 
1. der Inhalte und Erfolgschancen der voraussichtlichen strategischen Schritte eines jeden Wettbewerbers,

2. der zu erwartenden Reaktion jedes Wettbewerbers auf das Bündel strategischer Schritte, die andere Unternehmen initiieren könnten,

3. der wahrscheinlichen Reaktion jedes Wettbewerbers auf die Vielzahl der möglichen Veränderungen der Branche und des weiteren Umfeldes. ${ }^{114}$

Ausgehend von der Zielsetzung, die strategischen Schritte der Konkurrenten prognostizieren zu können, entwickelt PORTER ein System zur Konkurrenzanalyse, das eine begründete Vorhersage des Reaktionsprofils des Konkurrenten erlauben soll. Dieses Konzept wird im 3. Kapitel, Abschnitt 1.3.4, zusammen mit weiteren Inhalten und Instrumenten der Konkurrenzanalyse vorgestellt.

\subsubsection{Abgrenzung der Konkurrenzanalyse von verwandten analytischen Ansätzen}

Im Folgenden ist die Konkurrenzanalyse von den Inhalten des Benchmarking und der Marktforschung abzugrenzen.

In der Konkurrenzanalyse werden Informationen über Wettbewerber beschafft und verarbeitet. Sie ist neben der Betrachtung der Branchenstruktur und der darin vertretenen strategischen Gruppen das dritte Instrument, mit dessen Hilfe das Wettbewerbsumfeld eines Unternehmens analysiert werden kann. Innerhalb des strategischen Planungsprozesses erhalten diese Informationen eine grundlegende Bedeutung, weil auf ihrer Basis Entscheidungen gefällt werden. ${ }^{115}$

Während die Konkurrenzanalyse ausschließlich relevante Wettbewerbsunternehmen fokussiert, werden beim Benchmarking, je nach inhaltlicher Ausrichtung und betrachtetem Gegenstand, eigene Leistungen oder Abläufe systematisch und in einem partnerschaftlichen, detaillierten Analyseprozess mit denen anderer Unternehmen verglichen. Das Ziel ist es, vorbildliche Problemlösungen bei den beteiligten Referenzuntemehmen zu finden und sie auf die 
eigenen Gegebenheiten zu übertragen, um nachhaltige Verbesserungen zu erreichen. Die eigenen Leistungen oder Abläufe gilt es, nach dem Vorbild herausragender und exzellenter Praktiken zu optimieren. ${ }^{116}$

FRANZ/KAJÜTER zählen unterschiedliche Formen des Benchmarking auf. ${ }^{117}$ Sie unterscheiden zwischen dem Gegenstand einer Benchmarking-Analyse, bspw. Produkten, Prozessen und Methoden betrieblicher Funktionsbereiche, und der inhaltlichen Ausrichtung der Untersuchung, bspw. den Kosten-, Qualitäts-, Zeitund Kundenzufriedenheits-Benchmarks.

Darüber hinaus können die Vergleichspartner unterschieden werden:118

- Internes Benchmarking, bei dem die Vergleichspartner, bspw. mehrere Filialen, Werke oder Niederlassungen, aus dem gleichen Unternehmen stammen.

- Wettbewerbsorientiertes Benchmarking, bei dem direkte Konkurrenten in festgelegten Bereichen im Rahmen eines Benchmarking-Projektes zusammenarbeiten.

- Best-Practice Benchmarking, das das "Messen am Besten“ in den Mittelpunkt stellt, wobei unabhängig von der Branchenzugehörigkeit bestmögliche Problemlösungen oder Verbesserungen gesucht werden.

Benchmarking ist damit vielschichtiger und weiter gefasst als die reine Konkurrenzanalyse. ${ }^{119}$ Letztere kann jedoch nicht isoliert betrachtet werden, sondern ist im Kontext des strategischen Planungsprozesses zu sehen. ${ }^{120}$ In diesem stellt sie ein Analyseinstrument dar, das speziell Wettbewerbsunternehmen fokussiert und daher, im Gegensatz zum partnerschaftlich angelegten Benchmarking, einen eindeutig kompetitiven Charakter aufweist.

Auch beim Benchmarking erkennt man durch den Vergleich mit anderen Unternehmen relative Stärken und Schwächen. Allerdings lassen sich eigene Fähigkeiten, Prozessabläufe oder gar Kostenstrukturen im partnerschaftlichen Rahmen des Benchmarking normalerweise nur begrenzt mit denen direkter Wettbe-

$116 \mathrm{Vgl.} \mathrm{FRANZ/KAJÜTER,} 2000$ (a), S. 126.

$117 \mathrm{Vgl}$. FRANZ/KAJÜTER, 2000 (a), S. 126.

118 Vgl. FRANZ/KAJÜTER, 2000 (a), S. 126 und ähnlich: THOMMEN/ACHLEITNER, 1998, S. 837.

$119 \mathrm{Vgl}$. KAJÜTER, 2000, S. $120 \mathrm{ff}$.

120 Vgl. 1. Kapitel, Abschnitt I.1.3. 
werber vergleichen. Oft sind nur außenstehende, branchenfremde Unternehmen dazu bereit, interne Informationen im Kreis der Benchmarking-Partner detailliert offen zu legen, speziell dann, wenn es sich um kostenrelevante Angaben handelt. Werden jedoch solche Informationen zur strategischen Entscheidungsfindung benötigt, lassen sie sich meist eher durch eine entsprechende Konkurrenzanalyse als durch ein Benchmarking-Projekt beschaffen.

Die Marktforschung, in Abgrenzung zur Konkurrenzanalyse, dient der Erhebung von Marktdaten, die die Voraussetzung für das Treffen von Entscheidungen auf Beschaffungs- und auf Absatzmärkten sind. ${ }^{121}$ Ähnlich definiert BöHLER die Marktforschung als „systematische Sammlung, Aufbereitung, Analyse und Interpretation von Daten über Märkte und Marktbeeinflussungsmöglichkeiten zum Zweck der Informationsgewinnung für Marketing-Entscheidungen"122. KOTLER sieht die Marktforschung als Verbindung zwischen der Marketingabteilung eines Unternehmens und den Verbrauchern, den Kunden und der Öffentlichkeit. ${ }^{123}$ Weiter liefert die Marktforschung Informationen, die benötigt werden, „um

- Marketingchancen und Marketingprobleme zu erkennen,

- Marketingaktionen zu entwerfen, zu verfeinern und auf ihre Wirksamkeit zu überprüfen,

- die Wirkung des Marketings zu messen,

- das Marketinggeschehen zu verstehen"124.

Während die Konkurrenzanalyse im Bereich der strategischen Planung anzusiedeln ist, wird die Marktforschung eher dem Marketingbereich zugerechnet und ist damit nicht Gegenstand dieser Ausarbeitung.

Nach der begrifflichen Klärung und der Abgrenzung der Konkurrenzanalyse von verwandten analytischen Ansätzen, werden im 3. Kapitel die Inhalte und Instrumente der Konkurrenzanalyse vorgestellt.

\footnotetext{
$121 \mathrm{Vgl}$. NIESCHLAG/DICHTL/HÖRSCHGEN, 1991, S. 607; WÖHE, 1996, S. $608 \mathrm{ff}$.

122 BÖHLER, 1992, S. 17.

$123 \mathrm{Vgl}$. KOTLER, U.a., 1999, S. 238.

124 KOTLER, U.a., 1999, S. 238.
} 


\section{Kapitel Inhalte und Instrumente der Konkurrenzanalyse}

Eine wesentliche Komponente des marktwirtschaftlichen Systems ist der Wettbewerb zwischen mehreren Unternehmen einer Branche. Mit Hilfe der Konkurrenzanalyse werden das Leistungsangebot und die geschäftlichen Aktivitäten der Wettbewerber analysiert. Wie im 1. Kapitel dargelegt, benötigt man diese Kenntnisse, um darauf aufbauend eine eigene Unternehmensstrategie entwickeln zu können und um damit eine Fehlallokation der eigenen Ressourcen verhindern zu können. ${ }^{125}$

Unternehmen sind durch eine Fülle, auch von außen sichtbarer Merkmale charakterisiert. In der Konkurrenzanalyse werden alle Daten von Wettbewerbern betrachtet, die für eigene Entscheidungen im Rahmen der strategischen Unternehmensplanung von Bedeutung sind. ${ }^{126}$ Hierzu stehen eine Reihe von Instrumenten zur Verfügung, die jeweils bestimmte Merkmale von Wettbewerbern fokussieren. Dabei orientiert man sich häufig an internen Analysefeldern, mit denen nicht nur das eigene Unternehmen, sondern auch Konkurrenzunternehmen betrachtet werden.

Die vorgestellten Instrumente der Konkurrenzanalyse erfassen teilweise völlig unterschiedliche Inhalte, d.h. Merkmale der Wettbewerber. Alleinstehend sind daher viele Instrumente nicht in jeder Situation ausreichend, um ein genügend umfassendes Bild von Wettbewerbern zeichnen zu können. Vielmehr sind in Abhängigkeit von den individuellen Informationsbedürfnissen bestimmte Instrumente der Konkurrenzanalyse zu kombinieren.

Im Folgenden werden Instrumente und Inhalte der Konkurrenzanalyse dargestellt, wobei sich die Betrachtung darauf beschränkt, das Wesentliche herauszustellen, das im weiteren Verlauf der Untersuchung von Bedeutung ist. Dabei wird die Betrachtung von Kosten in der Konkurrenzanalyse auch durch die Auswahl der erwähnten Beispiele herausgestellt, falls es das jeweilige Instrument ermöglicht.

$125 \mathrm{Vgl}$. KREIKEBAuM, 1997, S. $121 \mathrm{f}$.

126 Vgl. KREIKEBAUM, 1997, S. 121. 


\subsubsection{Stärken-/Schwächen-Analyse und Konkurrenzanalyse}

Die Konkurrenzanalyse ist eine Voraussetzung für die Durchführung der Stärken-/Schwächen-Analyse, da hier eigene Unternehmensmerkmale u.a. an denen der Wettbewerber gemessen werden. ${ }^{127}$

Inhaltlich gleichen sich häufig beide Bereiche, weshalb die Stärken-/Schwächen-Analyse an dieser Stelle auch erwähnt werden soll. Denn prinzipiell sollen diejenigen Daten über die Konkurrenten erhoben werden, die auch Gegenstand der Potentialanalyse oder der Stärken-/Schwächen-Analyse innerhalb des eigenen Unternehmens sind. ${ }^{128}$ Die Stärken-/Schwächen-Analyse dient somit oft als Orientierung für die Inhalte der Konkurrenzanalyse.

Ein Beispiel für dieses Vorgehen liefert SCHREYÖGG129 in Anlehnung an HOFER/ SCHENDEL ${ }^{130}$. Bei der Ressourcenanalyse wird eine Funktions-/RessourcenMatrix erstellt, in der für die betrieblichen Funktionsbereiche F\&E, Produktion, Marketing, Finanzwirtschaft und Management folgende fünf Arten von Ressourcen erfasst werden:

1. Finanzielle Ressourcen: Cashflow, Kreditwürdigkeit, etc.. Die finanziellen Ressourcen werden als Basisressourcen betrachtet, weil sie in die übrigen Ressourcen transformiert werden können.

2. Physische Ressourcen: Gebäude, Anlagen, etc..

3. Humanressourcen: Facharbeiter, Führungskräfte, etc..

4. Organisatorische Ressourcen: Informationssysteme, Integrationsabteilungen, etc..

5. Technologische Ressourcen: Qualitätsstandard, F\&E-Know-how, etc..

Sind für jeden Funktionsbereich die genannten fünf Ressourcenarten erfasst und zugeordnet, werden diese den spezifischen Anforderungen des Marktes gegenübergestellt. Das Ziel ist es, Stärken und Schwächen in Bezug auf bestimmte Anforderungen des Marktes zu identifizieren.

\footnotetext{
127 Vgl. KREIKEBAUM, 1997, S. 121.

128 Vgl. KREIKEBAuM, 1997, S. 122; GÄLWEILER, 1986, S. 371.

$129 \mathrm{Vgl}$. SCHREYÖGG, 1984, S. $111 \mathrm{ff}$.

$130 \mathrm{Vgl}$. HOFER/SCHENDEL, 1978, S. $144 \mathrm{f}$.
} 
Im dritten Schritt dieses Schemas werden die ermittelten eigenen Stärken und Schwächen, die aus der oben dargestellten Gegenüberstellung von marktspezifischen Ressourcenanforderungen und der unternehmensspezifischen Ressourcenausstattung resultieren, mit denen der stärksten Konkurrenten verglichen. Hier ist es das Ziel, unter Berücksichtigung von Marktanforderungen Bereiche zu identifizieren, in denen man Wettbewerbsvorteile besitzt, auf denen Strategien aufgebaut werden können. ${ }^{131}$

Ein prinzipiell ähnliches Vorgehen wählt HAMMER ${ }^{132}$. In einem Stärken-/Schwächen-Profil werden die eigenen zu analysierenden Unternehmensmerkmale bewertet und anschließend mit denen eines oder mehrerer Hauptkonkurrenten verglichen. Bei der Ermittlung der zu erfassenden Merkmale orientiert sich HAMMER an zwei Fragestellungen, die unternehmensspezifisch zu bedenken sind:

1. Welche Einflussgrößen bestimmen jetzt oder in absehbarer Zukunft den Erfolg einer Unternehmung in einem bestimmten Sektor?

2. Was sind die Faktoren in einem Sektor, an denen man ansetzen muss, um seine zukünftige Wettbewerbsposition zu verbessern?

Global und in einem generellen Kontext nennt HAMMER die Faktoren, die einem repräsentativen Querschnitt in der Literatur entsprechen und die in Abbildung 9 dargestellt sind.

Bezüglich der Kostensituation betrachtet HAMMER die Produktions-, Vertriebsund Verwaltungsgemeinkosten, die Beschaffungskosten, Kosten für die Lagerhaltung, Personalkosten und Rationalisierungsmöglichkeiten. HAMMER zählt damit zu den Autoren, die Kosten von Wettbewerbern im Rahmen der Konkurrenzanalyse berücksichtigen, wie noch im 5 . Kapitel zu zeigen sein wird.

131 SCHREYögG gibt weitere Quellen an: Vgl. CHRISTENSEN/ANDREWS/BOWER, 1973, S. 237; TOURANGEAU, 1981, S. 85 ff.; GRANT/KING, 1982, S. 40 ff.; GLUECK, 1980, S. 154 ff. $132 \mathrm{Vgl}$. HAMMER, 1995, S. $40 \mathrm{ff}$. 


\begin{tabular}{|c|c|c|c|c|c|c|c|c|c|c|c|}
\hline \multirow{2}{*}{ Erfolgsfaktoren } & \multirow[t]{2}{*}{ Bewertung } & \multicolumn{3}{|c|}{ Schlecht } & \multicolumn{4}{|c|}{ Mittel } & \multicolumn{3}{|c|}{ Gut } \\
\hline & & 1 & 2 & 3 & 4 & 5 & 6 & 7 & 8 & 9 & 10 \\
\hline \multicolumn{2}{|c|}{$\begin{array}{l}\text { Produktions- u. Verkaufsprogramm } \\
\text { (Sortiment, Preis, Qualität, etc.) }\end{array}$} & & Q & & & & Q & & & & \\
\hline \multicolumn{2}{|c|}{$\begin{array}{l}\text { Produktionspotential (Modernität der } \\
\text { Verfahren u. Anlagen, Kapazität, etc.) }\end{array}$} & & & & & & b & & & & \\
\hline \multicolumn{2}{|c|}{$\begin{array}{l}\text { Vertriebspotential (Organisation, } \\
\text { Personal, Kapazität, etc.) }\end{array}$} & & & & & & & & & & \\
\hline \multicolumn{2}{|c|}{$\begin{array}{l}\text { Forschung und Entwicklung (Budget, } \\
\text { Ausstattung, Personal, etc.) }\end{array}$} & & $\alpha$ & & & & & & & & \\
\hline \multicolumn{2}{|c|}{$\begin{array}{l}\text { Einkaufspotential (Lieferantenstruktur, } \\
\text { Organisation d. Einkaufs, etc.) }\end{array}$} & & & & & & & & & & \\
\hline \multicolumn{2}{|c|}{$\begin{array}{l}\text { Personal (Qualifikation, Motivation, } \\
\text { Bedarfsdeckung, etc.) }\end{array}$} & & & & & & & & & & \\
\hline \multicolumn{2}{|c|}{$\begin{array}{l}\text { Standort (Infrastruktur, Marktnähe, } \\
\text { Erweiterungsmöglichkeiten, etc.) }\end{array}$} & & & & & & Q & & & & \\
\hline \multicolumn{2}{|c|}{ Kostensituation (siehe Ausführungen) } & & & & & & & 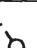 & & & \\
\hline \multicolumn{12}{|c|}{$\begin{array}{l}\text { Finanzielles Potential (Gesellschafts- } \\
\text { form, Selbstfinanzierungskraft, etc.) }\end{array}$} \\
\hline \multicolumn{12}{|c|}{$\begin{array}{l}\text { Führungssystem (Planung, Betriebs- } \\
\text { klima, Organisation, etc.) }\end{array}$} \\
\hline \multicolumn{2}{|c|}{$\begin{array}{l}\text { Produktivität (ungenutzte Potentiale, } \\
\text { Input-Output-Relation, etc.) }\end{array}$} & & & & & & & ○ & & & \\
\hline $\begin{array}{l}\text { Eigenes Unternehmen: } \\
\text { Konkurrenzunternehmen: }\end{array}$ & - & & & & & & & & & & \\
\hline
\end{tabular}

Abbildung 9: Das Stärken-/Schwächen-Profil nach HAMMER ${ }^{133}$

\subsubsection{Checklisten in der Konkurrenzanalyse}

Der Schritt vom oben beschriebenen Stärken-/Schwächen-Profil zu einer Vielzahl unterschiedlicher Checklisten, Kataloge und Arbeitsblättern liegt nahe. Diese, in der Praxis häufig verwendeten Listen, können den individuellen Informationsbedürfnissen eines Unternehmens angepasst werden. ${ }^{134}$ 
Als wesentlich erachtet GäLWEILER folgende Informationen, die im Rahmen einer Checkliste erfasst werden sollten: ${ }^{135}$ den Umsatz im Inland und im Ausland, die Anzahl der Beschäftigten, die vorhandenen Kapazitäten, die Marktanteile, die Programmbreite, die Standortvorteile und die Eigenschaften der Konkurrenzprodukte positiver oder negativer Art.

Weitaus detaillierter ist die von HOFFMANN vorgeschlagene Checkliste:136

\begin{tabular}{|l|l|}
\hline Management & Marketing \\
\hline - Führungskräfte & Verkaufsmannschaft \\
- Ziele und Prioritäten & - Fähigkeiten \\
- Wertvorstellungen & - Größe \\
- Entlohnungssysteme & - Art \\
Entscheidungsfindung & - Standorte \\
- Ort & Verteilernetz \\
- Art & Marktforschung \\
- Geschwindigkeit & - Qualität \\
Planung & - Struktur \\
- Arten & Service- und Verkaufsstrategie \\
- Engagement & Werbung \\
- Zeithorizont & - Qualität \\
Mitarbeiter & - Art \\
- Betriebszugehörigkeit & Mitarbeiter \\
- Fluktuation & - Führungskräfte/Fähigkeiten \\
- Erfahrung & - Fluktuation \\
- Beförderungspolitik & Finanzielle Mittel \\
Organisation & - Gesamt \\
- Zentralisation & - Kontinuität \\
- Funktionen & - \% des Umsatzes \\
- Nutzung der Stäbe & - Entlohnungssystem \\
\hline
\end{tabular}

$135 \mathrm{Vgl}$. GÄLWEILER, 1986, S. $370 \mathrm{ff}$.

136 Vgl. HoffmanN, 1986, S. $202 \mathrm{f}$. 


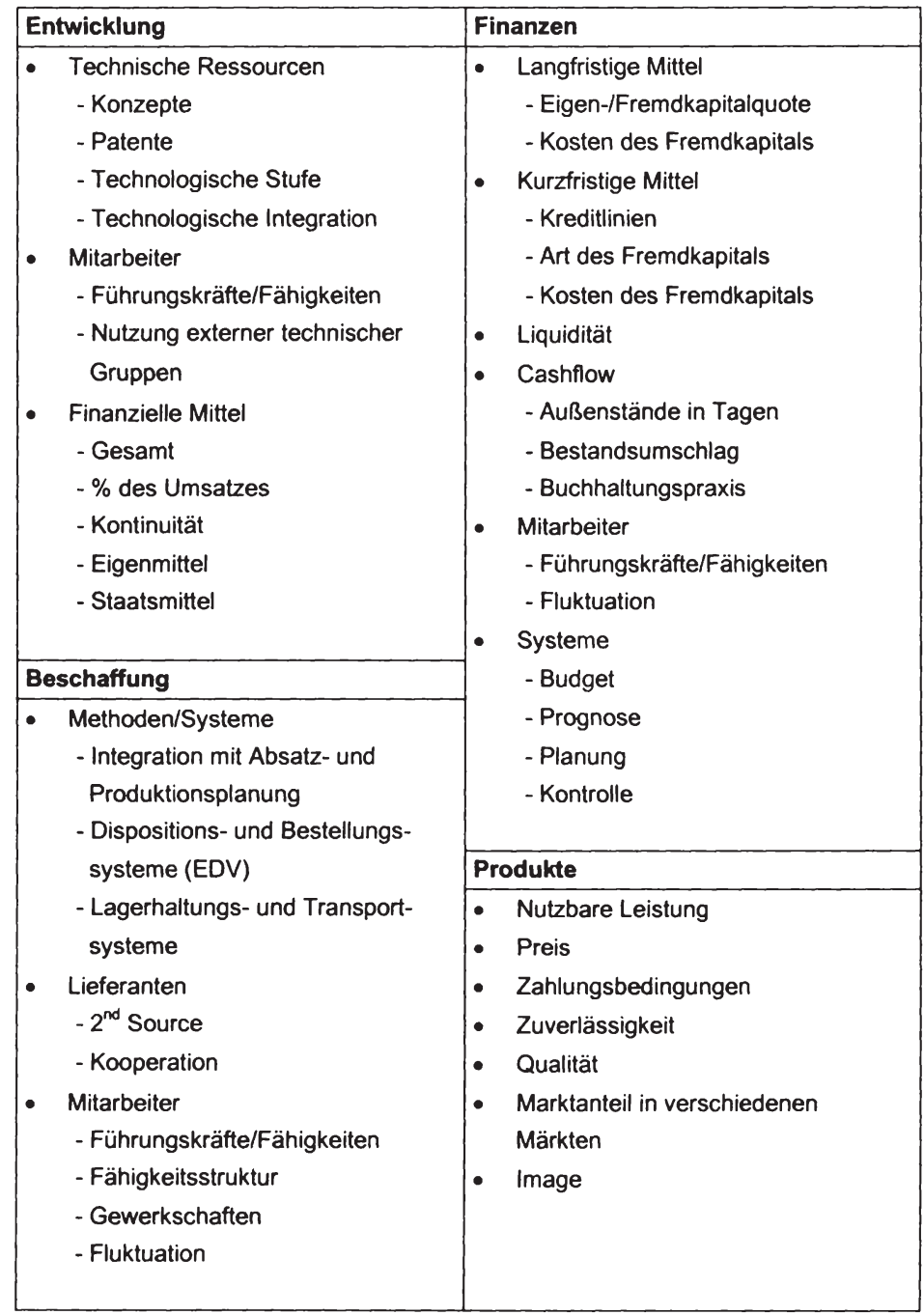

Abbildung 10: Die Checkliste zur Konkurrenzanalyse nach HOFFMANN ${ }^{137}$

Die in Abbildung 10 dargestellte Checkliste von HoffmanN bildet "wichtige Elemente einer Stärken-/Schwächen-Analyse der Konkurrenz"138 ab. Hierbei wer- 
den die zu erfassenden Inhalte in sechs Bereiche unterteilt: Management, Marketing, Entwicklung, Beschaffung, Produkte und Finanzen.

Die von HOFFMANN vorgeschlagene Checkliste sei hier exemplarisch nicht nur wegen ihres Detaillierungsgrades erwähnt, sondern auch weil sie trotz aller Ausführlichkeit keine Aussage zu Kosteninformationen von Konkurrenzunternehmen macht. ${ }^{139}$

Checklisten lassen sich produkt- oder marktspezifisch sehr detailliert bestimmten Einzelfällen anpassen. Einen spezifischen „Variablenkatalog zur Charakterisierung von Konkurrenzunternehmen"140 zeigt LINK, auf den an dieser Stelle nur hingewiesen werden soll. Bei den dort berücksichtigten Merkmalen steht der Absatzbereich im Mittelpunkt der Betrachtung. Gegliedert nach den Unternehmensfunktionen Allgemeine Firmenvariablen, Ertrags- und finanzwirtschaftliche Variablen, Variablen aus F\&E, Beschaffung und Produktion, sowie Variablen aus dem Absatzbereich, wird ein breites Spektrum analysiert, wobei der Absatzbereich sehr detailliert behandelt wird.

Einige Checklisten bestehen vollständig oder zu einem Großteil aus komplexen Fragestellungen, die über die Analyse einzelner Stichworte weit hinaus gehen. Die Stärke dieser Fragenkataloge besteht darin, Hintergründe zu beleuchten, Begründungen zu suchen und Zusammenhänge zu hinterfragen. Der Fragenkatalog von LINK wird in Abbildung 11 exemplarisch dargestellt141, da dort die Ermittlung von Kostenvorteilen im Rahmen der Konkurrenzanalyse schwerpunktmäßig untersucht wird, worauf im weiteren Verlauf dieser Arbeit an verschiedenen Stellen noch detaillierter eingegangen wird. ${ }^{142}$

Checklisten werden in der unternehmerischen Praxis häufig angewandt. Sie sind flexibel und können einfach auf die individuellen Informationsbedürfnisse eines Unternehmens angepasst werden, da sie nicht zwingend ein komplexes und zusammenhängendes System abbilden, wie dies bspw. bei einer Wert-

139 Auf den Themenbereich der Kostenbetrachtung in der Konkurrenzanalyse wird im 5. Kapitel genauer eingegangen.

140 LINK, 1988, S. 86.

141 Vgl. LINK, 1988, S. 128.

142 Zwei weitere Fragenkataloge, auf die an dieser Stelle nur hingewiesen werden soll, finden sich bei GROSS, 1974 und bei RASCHE, 1978. 
ketten- oder Portfolio-Analyse der Fall ist. ${ }^{143}$ Man kann sich bei Checklisten auf bestimmte Merkmale der Wettbewerber konzentrieren und durch gezielte Fragestellungen einzelne Hintergründe und Zusammenhänge analysieren.

\section{Fragen zur Ermittlung von Kostenvorteilen}

- Handelt es sich um eine Branche, in der niedrige Kosten eine maßgebende Wettbewerbsfähigkeitskomponente darstellen?

- Stellt die Betriebsgrößendegression eine wesentliche Barriere für neue Wettbewerber dar?

- Hat der Anbieter eine günstige Position auf der Erfahrungskurve?

- Verfügt der Anbieter über mengenbezogene Marktführerschaften?

- Kommen Vorteile bei der Beschaffung von Roh-, Hilfs- und Betriebsstoffen zum Tragen?

- Hat der Anbieter Standortvorteile?

- Verfügt das Unternehmen über Kostenvorteile bei der Fertigung?

- Gibt es Kostenvorteile im Fracht- und Lagerwesen?

- Sind die Lagerhaltungskosten im Wettbewerbsvergleich niedrig?

- Hat das Unternehmen im Branchenvergleich niedrige Blockkosten?

- Weist das Unternehmen bei der Kapitalbeschaffung Vorteile auf?

- Findet eine Konzentration auf Großserien und Produkte mit hohen Standardisierungsmöglichkeiten statt?

- Hat das Unternehmen im Branchenvergleich niedrige Finanzierungskosten?

Abbildung 11: Ein Fragenkatalog zur Ermittlung von Kostenvorteilen ${ }^{144}$

\subsubsection{Bilanzanalyse im Rahmen der Konkurrenzanalyse}

Als Bilanzanalyse, synonym wird der Begriff Jahresabschlussanalyse verwendet, bezeichnet COENENBERG „Verfahren der Informationsgewinnung und -auswertung, mit deren Hilfe aus den Angaben des Jahresabschlusses - Bilanz, Gewinn- und Verlustrechnung, Kapitalflussrechnung und Anhang - und des Lageberichtes Erkenntnisse über die Finanz- und Ertragslage der Unternehmung gewonnen werden“145. Große Kapitalgesellschaften sind verpflichtet, einen

143 Die Wertkette und die Portfolio-Analyse werden in diesem Kapitel ausführlich in den Abschnitten 1.3.6 und 1.3.7 dargestellt.

$144 \mathrm{Vgl}$. LINK, 1988, S. 128.

145 COENENBERG, 2000, S. 873. 
Jahresabschluss, bestehend aus Bilanz, Gewinn- und Verlustrechnung, Anhang und Lagebericht zu erstellen. ${ }^{146}$ Der Jahresabschluss muss gemäß $\S 264$ Abs. 2 HGB „unter Beachtung der Grundsätze ordnungsgemäßer Buchführung ein den tatsächlichen Verhältnissen entsprechendes Bild der Vermögens-, Finanzund Ertragslage"147 vermitteln.

Die Bilanzanalyse ist mit ihrem zur Verfügung stehenden Zahlenmaterial „eine der verbreitetsten Informationsquellen“, um „eine gewisse Kenntnis der Wettbewerbsposition verschiedener Unternehmen in einer Branche zu erlangen"148.

Den Stellenwert, den die Jahresabschlussanalyse von Wettbewerbsunternehmen im Rahmen der Konkurrenzanalyse einnimmt, behandelt ausführlich GRÜTTER-SeTtele. ${ }^{149}$ Demnach bewertet eine Mehrzahl der betrachteten Autoren ${ }^{150}$ "den Jahresabschluss als potentielle Entscheidungsgrundlage für die Konkretisierung einer Unternehmensstrategie"151. Diese Aussage wird von GRÜTTER-SETTELE empirisch bestätigt. ${ }^{152}$

Sehr detailliert behandelt COENENBERG die Inhalte der Jahresabschlussanalyse. ${ }^{153}$ Bei der Finanzwirtschaftlichen Bilanzanalyse unterscheidet er drei wesentliche Bereiche:

1. Investitionsanalyse, d.h. die Struktur des Vermögens

- Verhältnis von Anlage- zu Umlaufvermögen

- Umsatzrelationen

- Umschlagskoeffizienten

- Kennzahlen zur Untersuchung der Investitions- und Abschreibungspolitik

2. Finanzierungsanalyse, d.h. die Kapitalstruktur

- Verschuldungsgrad

- Weitere Kennzahlen zur Kapitalstruktur

$146 \S 267$ Abs. 1 bis 3 HGB.

$147 \S 264$ Abs. 2 HGB.

148 HAX/MAJLUF, 1991, S. 286.

$149 \mathrm{Vgl}$. GRÜTtTER-SetTELE, 1999, S. $10 \mathrm{ff}$.

150 Vgl. Coenenberg, 2000, S. 875 f.; Weber, 1987, S. 105 f.; BIeg/KubMaul, 1996, S. 17 ff.; HÖFLICH, 1982, S. $108 \mathrm{ff}$.

151 GRÜTTER-SETTELE, 1999, S. 16.

152 Vgl. GRÜTTER-SETTELE, 1999, S. $193 \mathrm{ff.}$

153 Vgl. COENENBERG, 2000, S. 871 bis 1049. 
3. Liquiditätsanalyse, d.h. der Zusammenhang zwischen Investitionen und Finanzierung
a) Liquiditätsanalyse aufgrund von Bestandsgrößen
- Fristenkongruenz
- Kurz-, mittel- und langfristige Liquiditätskennzahlen
b) Liquiditätsanalyse aufgrund von Stromgrößen
- Umsatzüberschusskennzahlen, d.h. Cashflow-Analysen
- Kapitalflussrechnungen
c) Liquiditätsanalyse durch kombinierte Darstellung von Bestands- und Stromgrößen

Bei der Erfolgswirtschaftlichen Bilanzanalyse steht die Ertragskraft im Fokus, wobei COENENBERG vier Analysefelder unterscheidet:

1. Ergebnisanalyse

a) Betragsmäßige Ergebnisanalyse

- Auswertung von Informationen des Anhangs

- Geschätzter Steuerbilanzgewinn als Indikator der Ertragskraft

- Cashflow als Indikator der Ertragskraft

b) Strukturelle Ergebnisanalyse

- Ergebnisquellenanalyse

- Analyse der Aufwands- und Ertragsstruktur

2. Rentabilitätsanalyse

3. Wertschöpfungsanalyse

4. Break-even-Analyse

COENENBERG berücksichtigt bei der Jahresabschlussanalyse die Interessen aller Adressaten des Jahresabschlusses, also auch die von Wettbewerbsunternehmen. Dabei unterscheidet er insgesamt zwei Adressatengruppen: ${ }^{154}$

1. Adressaten, die durch ein vertraglich fixiertes Schuldner-Gläubiger-Verhältnis mit dem Unternehmen verbunden sind und die sich besonders für die Vermögens- und Finanzlage interessieren (Gläubiger, Lieferanten, Kunden, Arbeitnehmer, etc.) und 
2. Adressaten, die größeres Interesse an der Ertragskraft des Unternehmens haben (Aktionäre, Gewerkschaften, Konkurrenten, etc.).

„Der Konkurrenz dienen Ertragskraft-Daten als Vergleichsmaßstab für die Beurteilung der eigenen Ertragslage. So können bspw. Informationen gewonnen werden, ob das Produktionsprogramm der Konkurrenz einen höheren Ertrag abwirft als das eigene, oder ob ein Preiskampf Aussicht auf Erfolg verspricht." ${ }^{155}$

Im Gegensatz zu COENENBERG ordnen HAX/MAJLUF ihren Beitrag zur Bilanzanalyse, der einen deutlich geringeren Detaillierungsgrad aufweist, dem Bereich Branchen- und Wettbewerbsanalyse zu. Sie nennen zwei Verfahren, um Bilanzzahlen verschiedener Wettbewerber vergleichbar zu machen: den Vergleich der Bilanzstruktur und die Kennzahlenanalyse. ${ }^{156}$ Beim Vergleich der Bilanzstruktur mehrerer Unternehmen können die unterschiedlichen absoluten Bilanzzahlen auf einen einheitlichen Wert - in der Regel 100 - normiert werden, wodurch man jedoch bereits Kennzahlen erhält, die die prozentuale Aufteilung aller Bilanzpositionen zeigen. Beim Kennzahlenvergleich, der "verbreitetsten Form der

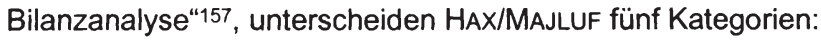

1. Liquiditätskennzahlen,

2. Kapitalstruktur-Kennzahlen,

3. Rentabilitätskennzahlen,

4. Umschlagkennzahlen,

5. Kennzahlen zur Aktienanalyse.

Neben diesen fünf Kategorien von Finanzkennzahlen werden oft branchenspezifische Leistungsmerkmale aus dem Jahresabschluss für die Konkurrenzanalyse verwendet. Beispiele dafür sind: ${ }^{158}$

- die F\&E-Aufwendungen in Prozent vom Umsatz, evtl. aufschlussreich bei Hochtechnologie-Unternehmen mit kurzen Produkt-Lebenszyklen,

- das Verhältnis der Anlageinvestitionen zum Umsatz, evtl. interessant bei Branchen mit hoher Kapitalintensität,

155 Coenenberg, 2000 , S. 876.

$156 \mathrm{Vgl}$. HAX/MAJLUF, 1991, S. 286.

157 HAX/MAJLUF, 1991, S. 286.

158 Vgl. HAX/MAJLuF, 1991, S. 291. 
- das Unternehmenswachstum in Relation zum Branchenwachstum, evtl. als Indikator für Veränderungen des relativen Marktanteils einzelner Wettbewerber.

Zur Beurteilung der Daten - gleich ob absolute oder relative Bilanzzahlen, Kennzahlen oder branchenspezifische Leistungsmerkmale - werden Vergleichsmaßstäbe benötigt. ${ }^{159}$ Für HAX/MAJLUF sind die Querschnittsanalyse und die Zeitreihenanalyse die beiden wichtigsten Auswertungsmethoden der Bilanzanalyse. Bei der Querschnittsanalyse wird die Abweichung aller Unternehmen einer Branche von einer Bezugsgröße betrachtet, wofür gewöhnlich der arithmetische Mittelwert oder der Medianwert der Branche herangezogen werden. Bei der Zeitreihenanalyse steht die zeitliche Entwicklung eines Analysemerkmals im Vordergrund. „Der Zeitvergleich hat den Vorteil, dass die Wirkung bilanzpolitischer Maßnahmen, die eine Verschiebung oder Vorwegnahme von Ergebnissen bewirken, langfristig wieder aufgehoben werden."160 Andererseits besteht beim Zeitvergleich die Gefahr, eine in der Vergangenheit sichtbare Tendenz in die Zukunft zu extrapolieren, was wegen zunehmender Marktdynamik und sich ständig ändernder Umfeldbedingungen als unsicher zu bezeichnen ist. ${ }^{161}$

Der große Informationsgehalt des Jahresabschlusses für die Konkurrenzanalyse darf nicht über einige Grenzen der Jahresabschlussanalyse hinweg täuschen, die es trotz allem Nutzen zu bedenken gilt:

1. Während sich die Strategische Planung mit der zukünftigen Unternehmensentwicklung befasst, basiert der Jahresabschluss auf Zahlen der Vergangenheit, die nicht unbedingt extrapolierbar sind. Hinzu kommt, dass der Jahresabschluss erst einige Monate nach dem Bilanzstichtag verfügbar ist.

2. Der Jahresabschluss bildet - dargestellt in Geldeinheiten - vor allem die finanziellen Transaktionen eines Unternehmens ab. ${ }^{162}$ Qualitative Gesichtspunkte, die für eine Unternehmensbeurteilung bedeutend sein können, werden auf den Anhang und den Lagebericht beschränkt. Nicht monetär

$159 \mathrm{Vgl}$. HAX/MAJLUF, 1991, S. $291 \mathrm{ff}$.

160 COENENBERG, 2000, S. 893.

161 Vgl. Coenenberg, 2000, S. 876 f., ähnlich: GRütter-SetTele, 1999, S. 23 f., in Anlehnung an: ANSOFF, 1987, S. $12 \mathrm{f}$.

$162 \mathrm{Vgl}$. im Folgenden: CoenenBerg, 2000, S. 877. 
quantifizierbare Angaben, wie bspw. die Qualität des Managements, technisches Know-how, Entwicklungsprojekte oder die Marktstellung fehlen. Da der Jahresabschluss nur realisierte Aktivitäten abbildet, bleiben potentielle oder nicht zustande gekommene Transaktionen im Verborgenen, es sei denn, sie sind so wesentlich für die zukünftige Unternehmensentwicklung, dass sie im Lagebericht erwähnt werden.

3. Der Ausweis der Vermögens-, Finanz- und Ertragslage ist durch das Vorsichtsprinzip beeinflusst. Hinzu kommen diverse Maßnahmen, die durch Bilanzierungs- und Bewertungswahlrechte den Informationsgehalt des Jahresabschlusses reduzieren. ${ }^{163}$ Der bilanzpolitische Spielraum wird durch die Vorschriften des HGB, der IAS oder der US-GAAP definiert, worauf COENENBERG ausführlich eingeht. ${ }^{164}$

Als alleiniges Instrument der Konkurrenzanalyse ist die Bilanzanalyse damit in vielen Fällen nicht ausreichend. Hinzu kommt, dass viele kleine und mittlere Unternehmen nicht der Veröffentlichungspflicht unterliegen. Im Zusammenspiel mit anderen in diesem Abschnitt dargestellten Instrumenten stellt die Bilanzanalyse jedoch eine deutliche Erweiterung der Informationsbasis dar, wenn dieses Zahlenmaterial zu beschaffen ist.

\section{I.3.4 System zur Konkurrentenanlyse nach PORTER}

Während Kriterienkataloge und die Jahresabschlussanalyse ein eher vergangenheits- und gegenwartsbezogenes Bild von Konkurrenzunternehmen zeichnen, erlaubt das System zur Konkurrentenanalyse nach PORTER „eine begründete Vorhersage des Reaktionsprofils des Konkurrenten"165, d.h. die Prognose zukünftiger strategischer Aktionen und Reaktionen von Wettbewerbern.

Wie Abbildung $12 \mathrm{zu}$ entnehmen ist, bestimmen vier $\mathrm{zu}$ diagnostizierende Elemente das zukünftige Verhalten eines Konkurrenten: ${ }^{166}$

163 SCHNEIDER spricht in diesem Zusammenhang von Jahresabschlüssen als einer Mischung aus Dichtung und Wahrheit, vgl. SCHNEIDER, 1989, S. 633.

$164 \mathrm{Vgl}$. COENENBERG, 2000, S. $877 \mathrm{ff}$.

165 PORTER, 1999 (a), S. 87.

$166 \mathrm{Vgl}$. im Folgenden: PORTER, 1999 (a), S. $87 \mathrm{ff}$. 
Was den Konkurrenten motiviert

Ziele für die Zukunft

Auf allen Managementebenen und für verschiedene Gebiete
Wie sich der Konkurrent verhält und verhalten kann

Gegenwärtige Strategie Wie der Konkurrent zur Zeit den Wettbewerb führt

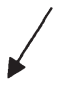

Reaktionsprofil des Konkurrenten

Ist der Konkurrent mit seiner gegenwärtigen Situation zufrieden?

Welche voraussichtlichen Schritte oder strategische

Veränderungen wird der Konkurrent vornehmen?

Wo ist der Konkurrent verwundbar?

Was wird die größte und wirkungsvollste Reaktion des Konkurrenten hervorrufen?

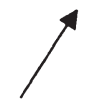

Annahmen

Über sich selbst und über die Branche
Fähigkeiten

Stärken und Schwächen

Abbildung 12: Das System zur Konkurrenzanalyse nach PORTER ${ }^{167}$

\section{Ziele für die Zukunft:}

Stellt man die Ziele von Wettbewerbern und die Art, wie sie diese verfolgen, der gegenwärtigen Position gegenüber, lassen sich daraus Rückschlüsse ableiten, ob und inwieweit ein Konkurrenzunternehmen mit den gegenwärtigen Verhältnissen zufrieden ist. Daraus lässt sich die Wahrscheinlichkeit für einen Strategiewechsel und die Intensität abschätzen, mit der dieser Wettbewerber auf äußere Einflüsse oder auf Maßnahmen anderer Unternehmen reagieren wird. Neben finanziellen Zielen umfasst diese Diagnose qualitative Faktoren, wie z.B. die Markt- oder Technologieführerschaft.

\section{Annahmen:}

Wie bei der Betrachtung zukünftiger Ziele fördert auch die Analyse der Annahmen, die ein Konkurrent über seine eigene Situation, die seiner Branche und die der Unternehmen in der Branche hat, ein Verständnis für sein Verhalten. Erkennt man dabei falsche Annahmen, wie bspw. eine falsche 
Einschätzung von Markt- oder Technologietrends oder eine überschätzte Kostenposition lassen sich daraus entsprechende Vorteile für das eigene Unternehmen generieren.

\section{Gegenwärtige Strategien:}

Während die Ziele und Annahmen die Motivation der Konkurrenten fokussieren, stehen bei den Strategien und den Fähigkeiten die Fragen im Vordergrund: wie verhält sich der Konkurrent und wie kann er sich verhalten. Die gegenwärtige Strategie des Konkurrenten entspricht seiner Kombination der Produkt-Markt-Aktionsparameter, die die Regeln des Wettbewerbs beeinflussen. ${ }^{168}$

\section{Fähigkeiten:}

Die Fähigkeit, strategische Schritte zu ergreifen, wird durch die Stärken und Schwächen eines Wettbewerbers beeinflusst. Bei der Stärken-/SchwächenAnalyse nutzt PORTER Checklisten und ergänzt diese durch zusätzliche verbindende Fragen. ${ }^{169}$ Inhaltlich geht PORTER u.a. ausführlich auf Kosten von Konkurrenzunternehmen ein. In diesem Zusammenhang betrachtet er bspw. die relative Gesamtkostensituation, d.h.

- mit anderen Geschäftseinheiten aufgeteilte Kosten bzw. gemeinsam betriebene Aktivitäten,

- Bereiche, in denen der Wettbewerber Größenvorteile oder andere Faktoren hervorbringt, die für seine Kostenposition entscheidend sind. 170

Zusätzlich stellt PORTER u.a. folgende Fragen, die die Anpassungsfähigkeit einzelner Unternehmen analysieren: ${ }^{171}$

- Welches Verhältnis besteht zwischen fixen und variablen Kosten?

- Wie hoch sind die Kosten ungenutzter Kapazitäten?

Die Synthese der vier Elemente ergibt das Reaktionsprofil des betrachteten Konkurrenten, das in Abbildung 13 dargestellt ist. Dabei werden nicht nur mögliche offensive Schritte des Wettbewerbers oder des eigenen Unternehmens

\footnotetext{
$168 \mathrm{Vgl.} \mathrm{WELGE/AL-LAHAM,} \mathrm{1999,} \mathrm{S.} 227$.

169 Vgl. PORTER, 1999 (a), S. $105 \mathrm{ff}$.

170 Vgl. PORTER, 1999 (a), S. 106.

$171 \mathrm{Vgl}$. PORTER, 1999 (a), S. $108 \mathrm{f}$.
} 
analysiert, sondern auch die Verwundbarkeiten und mögliche Abwehr- oder Vergeltungsmaßnahmen.

\begin{tabular}{|l|l|l|l|}
\cline { 2 - 3 } \multicolumn{1}{c|}{} & $\begin{array}{l}\text { Verwundbarkeit } \\
\text { des Konkurrenten } \\
\text { durch das Ereig- } \\
\text { nis }\end{array}$ & $\begin{array}{l}\text { Ausmaß, in dem } \\
\text { das Ereignis den } \\
\text { Konkurrenten zur } \\
\text { Vergeltung pro- } \\
\text { vozieren wird }\end{array}$ & $\begin{array}{l}\text { Wirksamkeit der } \\
\text { Vergeltungsmaß- } \\
\text { nahmen des } \\
\text { Konkurrenten }\end{array}$ \\
\hline $\begin{array}{l}\text { Mögl. strategische } \\
\text { Schritte des eige- } \\
\text { nen Unternehmens }\end{array}$ & $\begin{array}{l}\text { Alle Alternativen aufzählen, z.B.: } \\
- \text { Produktprogramm erweitern } \\
- \text { Qualität und Service verbessern }\end{array}$ \\
\hline $\begin{array}{l}\text { Denkbare Verände- } \\
\text { rungen des Um- } \\
\text { feldes }\end{array}$ & $\begin{array}{l}\text { Alle Veränderungen aufzenken u. Kostenwettbewerb führen } \\
- \text { Bedeutende Rohstoffverteuerungen }\end{array}$ \\
\hline
\end{tabular}

Abbildung 13: Das Reaktionsprofil des Konkurrenten ${ }^{172}$

PORTERS System zur Konkurrentenanalyse bereichert die bisher dargestellten Ansätze um die Motivationsfaktoren von Wettbewerbern. Durch die Berücksichtigung der Ziele und Annahmen versetzt man sich in die Lage des Konkurrenten und betrachtet die Situation aus seinem Blickwinkel. Mögliche zukünftige Aktionen und Reaktionen lassen sich auf diese Weise antizipieren. Kombiniert mit der gegenwärtigen strategischen Ausrichtung und den Fähigkeiten des Wettbewerbers können mit Hilfe dieser Methodik komplexe Szenarien entwickelt werden, die die Zukunft "planbarer" machen. Die Ungewissheit der strategischen Unternehmensplanung, die sich mit dieser Zukunft beschäftigt, wird dadurch reduziert.

\subsubsection{Erfahrungskurve als Instrument der Konkurrenzanalyse}

Die Grundlagen des Konzeptes der Erfahrungskurve, synonym wird der Begriff Kostenerfahrungskurve verwendet, gehen zurück auf Beobachtungen des Fliegers und Flugzeugproduzenten WHRIGHT im Jahre 1925, die später in der Mitte

172 Vgl. PORTER, 1999 (a), S. 112. 
der 60er-Jahre von der BOSTON CONSULTING GROUP in umfangreichen empirischen Studien weitergeführt wurden. ${ }^{173}$ Die zentrale Aussage besteht darin, dass "die auf den Wertschöpfungsanteil bezogenen und in konstanten, d.h. inflationsbereinigten Geldeinheiten ausgedrückten Stückkosten eines Produktes potentiell um 20 bis 30 Prozent zurückgehen, wenn die kumulierten Produktionsmengen verdoppelt werden ${ }^{\text {"1 }}{ }^{74}$.

Abbildung 14 stellt diesen Zusammenhang graphisch dar:

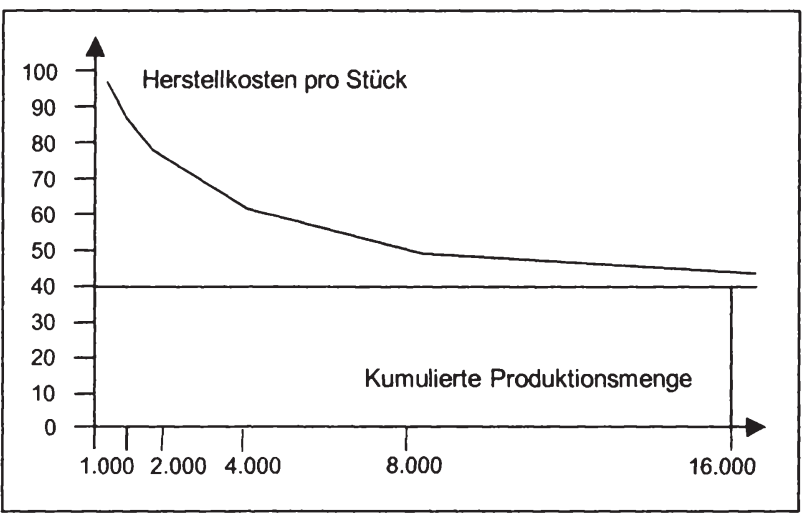

Abbildung 14: Die Erfahrungskurve

WELGE/AL-LAHAM zählen in Anlehnung an diverse Autoren ${ }^{175}$ folgende Ursachen des Erfahrungskurveneffektes auf: 176

1. Kostendegression durch zunehmende Betriebsgröße, wobei sich diese auf die Produktionsmenge eines Zeitraumes und nicht auf die bisherige kumulierte Gesamtproduktionsmenge eines Unternehmens bezieht,

2. Rationalisierung durch stetig verbesserte Verfahren und Arbeitsabläufe in allen Unternehmensbereichen,

3. Steigerung der Leistungsfähigkeit durch stetigen technischen Fortschritt.

$173 \mathrm{Vgl}$. WELGE/AL-LAHAM, 1999, S. $157 \mathrm{f}$.

174 WeLge/AL-LAHAM, 1999, S. 157, in Anlehnung an: HeNDERSON, 1984, S. 19.

175 Vgl. Hedley, 1997, S. 327 ff.; KREIKEBAUM, 1997, S. 98 ff.; HAMMER, 1995, S. 146 f.; HaXI MAJluf, 1991, S. 127 ff.; KREILKAMP, 1987, S. 337; HeNDERSON, 1984, S. 26 f.; GRIMM, 1983, S. $97 \mathrm{ff}$.; WACKER, 1980, S. $60 \mathrm{ff}$.

176 Vgl. WELGE/AL-LAHAM, 1999, S. $158 \mathrm{ff}$. 
Die Erkenntnisse aus dem Konzept der Erfahrungskurve finden Berücksichtigung bei der Auswahl der generellen Wettbewerbsstrategie. Die bereits erläuterte Strategie der Kostenführerschaft'177 basiert zu wesentlichen Teilen auf dem Erfahrungskurveneffekt, wofür das Erreichen einer beherrschenden Marktposition von besonderer Bedeutung ist. Dabei hat das Unternehmen mit dem größeren Marktanteil auf einem gegebenen, abgegrenzten Markt Kostenvorteile nur aufgrund seiner größeren kumulierten Produktionsmenge. ${ }^{178}$

Neben der Anwendung bei der Strategieformulierung wird die Erfahrungskurve „zur Kostenplanung, d.h. zur Kostenvorgabe und -steuerung“179 verwendet. Dabei setzt man die Erfahrungskurve üblicherweise ein, um Transparenz in die Dynamik der Kostenstruktur des eigenen Unternehmens zu bringen.

Obwohl sich die Erfahrungskurve nicht nur auf das eigene, sondern auch auf Konkurrenzunternehmen anwenden lässt, wird dieses Konzept im Allgemeinen nicht in Verbindung mit der Konkurrenzanalyse betrachtet. Die Erfahrungskurve stellt jedoch ein wichtiges Instrument dar, um „die Frage der wettbewerbsmäßigen Kostenstruktur formal“"180 anzugehen. So kann die Erfahrungskurve „wichtige Informationen über Markt- und Wettbewerbsverhältnisse liefern. Wenn es z.B. möglich ist, sekundärstatistisch den Erfahrungseffekt einer Branche abzuschätzen, so können, verfolgt man zugleich die Ausbringungsmengen der Konkurrenz, wichtige Einblicke in die Kostenhöhe und -struktur der Konkurrenz gewonnen werden."181

Die Konkurrenten-Erfahrungskurve, KREIKEBAUM bezeichnet sie als "Cross sectional experience-curve"182, kann für alle Wettbewerber identisch sein. Dies setzt voraus, dass die Kosten der ersten Einheit, sichtbar im Ursprung der Erfahrungskurve auf der Ordinate, und die Elastizität der Stückkosten, d.h. die Steigung der Erfahrungskurve, gleich sind. ${ }^{183}$ Unter der Annahme, dass sich die Absatzmengen und die kumulierten Produktionsmengen parallel entwickelt haben und das Marktvolumen und die Absatzmengen der einzelnen Anbieter

\footnotetext{
177 Siehe 1. Kapitel, Abschnitt I.1.3.3.

$178 \mathrm{Vgl}$. WELGE/AL-LAHAM, 1999, S. 160.

179 Welge/AL-LAHAM, 1999, S. 160.

180 HAX/MAULUF, 1991, S. 133.

181 Welge/AL-Laham, 1999, S. 160; PICOT, 1981, S. 563.

182 KREIKEBAUM, 1997, S. 106.

183 Vgl. KREIKEBAUM, 1997, S. 106.
} 
abschätzbar sind, ergeben sich Kostenabstände zwischen den Anbietern. ${ }^{184}$ Auf die strategische Bedeutung dieser Information wird im späteren Verlauf dieser Untersuchung ausführlich eingegangen.

Diverse Faktoren können zu einer Ungleichheit führen, so dass die Kostensituation bei alleiniger Betrachtung der Marktanteile falsch widergegeben wird:

- Unterschiedliche Zeitpunkte des Markteintritts führen aufgrund verschiedener Eintrittsbedingungen zu ungleichen Kosten der ersten Einheit.

- Die Wertschöpfungskette bestehen aus mehreren Phasen, z.B. F\&E, Beschaffung, Fertigung und Vertrieb. Bei jeder einzelnen Phase kommt es zu individuellen Erfahrungseffekten. Bspw. kann ein Anbieter im Konzernverbund seine F\&E-Aktivitäten konzentrieren, während ein anderes Unternehmen durch den Vertrieb mehrerer verwandter Produkte Vorteile im Sinne der Erfahrungskurve im Vertrieb besitzt. Durch diese Unterschiede in der Wertschöpfungskette kann die Elastizität, d.h. die Steigung der Erfahrungskurve bei Unternehmen der gleichen Branche differieren. ${ }^{185}$

- Außergewöhnlich schneller technischer Fortschritt oder signifikante Kapazitätserweiterungen verändern sprunghaft vorhandene Erfahrungseffekte und müssen damit in die Überlegungen einbezogen werden. ${ }^{186}$ Gleiches gilt für bedeutende Produkt- und Verfahrensinnovationen. Erschwerend kommt hinzu, dass die genannten Veränderungen in den Unternehmen zu unterschiedlichen Zeitpunkten durchgeführt werden.

Eine Kostenprognose von Konkurrenten unter Verwendung der Erfahrungskurve setzt demnach eine einzelfallspezifische Analyse der Kostenstruktur und deren Entwicklung voraus. Alle Einflussfaktoren sind zu identifizieren, damit durch eine sorgfältige Kausalanalyse der Kostendegressionseffekt - und damit die Kostenposition der einzelnen Anbieter - nachvollzogen werden kann.

KREIKEBAUM kritisiert die theoretische Fundierung des Erfahrungskurvenkonzeptes. ${ }^{187}$ Ein Effekt der Kostendegression lässt sich auf viele Einflussfaktoren zurückführen. Die kumulierte Produktionsmenge als „erklärende Variable“ reicht

$184 \mathrm{Vgl.} \mathrm{WELGE/AL-LAHAM,} \mathrm{1999,} \mathrm{S.} 160$.

$185 \mathrm{Vgl}$. HAX/MAJLUF, 1991, S. $145 \mathrm{ff}$.

$186 \mathrm{Vgl.} \mathrm{HAX/MAJLUF,} \mathrm{1991,} \mathrm{S.} 143$.

187 Vgl. KREIKEBAUM, 1997, S. 107 f.; LANGE, 1984, S. 238; DAY/MONTGOMERY, 1983, S. $46 \mathrm{ff.}$ 
alleine nicht aus, diesen Effekt theoretisch zu erklären. Da die verschiedenen Einflüsse und deren Wirkungen auf den Erfahrungskurveneffekt eng miteinander verknüpft sind und sich gleichgerichtet entwickeln, lassen sie sich entsprechend schwierig getrennt voneinander untersuchen. Aus Anwendungsgesichtspunkten wäre eine Trennung der verschiedenen Einflüsse wünschenswert, da je nach Bedeutung der einzelnen Einflussfaktoren unterschiedliche Maßnahmen erforderlich sind. ${ }^{188}$

In der Praxis sieht HENDERSON folgende Schwierigkeiten: 189

- Interne Kostendaten im benötigten Detaillierungsgrad sind Geschäftsgeheimnisse und daher oft nur schwer zugänglich. ${ }^{190}$

- Kostengegenüberstellungen müssen auf der Basis einheitlicher Kostenrechnungsverfahren in den analysierten Unternehmen erfolgen, was nicht zwangsweise gewährleistet werden kann.

- Eine Änderung der Kostenerfassung und -zurechnung im Periodenverlauf

Trotz aller Schwierigkeiten „ist nicht zu leugnen, dass die Erfahrungskurve für die Strategieplanung bedeutende Einsichten liefert"191, wobei ihr Gebrauch die Beachtung spezifischer Richtlinien erfordert.

\section{I.3.6 Wertkette in der Konkurrenzanalyse}

Das von PORTER entwickelte Konzept der Wertkette ist eine systematische Methode und ein „analytisches Instrument“192, mit dem ein Unternehmen in strategisch relevante Tätigkeiten gegliedert werden kann. ${ }^{193}$ Die Segmentierung in einzelne sog. Wertaktivitäten ermöglicht es, Kernkompetenzen, auszulagernde Tätigkeiten, aber auch, was hier von besonderem Interesse ist, spezifische Wettbewerbsvorteile im Vergleich zu Konkurrenten zu finden. ${ }^{194}$ Wertaktivitäten

\footnotetext{
188 Vgl. GHEMAWAT, 1985, S. $35 \mathrm{f}$.

189 Vgl. HENDERSON, 1984, S. 26.

190 Diese Thematik wird im 4. Kapitel ausführlich behandelt.

191 HAX/MAJLUF, 1991, S. 142.

192 PORTER, 1999 (b), S. 63.

193 Eine detaillierte Beschreibung des Aufbaus der Wertkette findet sich bei PORTER, 1992, S. $63 \mathrm{ff}$.

$194 \mathrm{Vgl}$. HINTERHUBER, 1996 (a), S. $179 \mathrm{f}$.
} 
werden oft mit Hilfe der Wertkette dargestellt und sind physisch und technologisch unterscheidbare Aktivitäten, die von einem Unternehmen zur Produktoder Leistungserstellung ausgeführt werden. ${ }^{195}$

Jedes Unternehmen einer Branche weist eine individuelle Wertkette auf, was auf Unterschiede in der Geschichte, den Strategien und den Erfolgen der jeweiligen Wettbewerber zurückzuführen ist. Indem es strategisch wichtige Aktivitäten „billiger oder besser"196 als seine Konkurrenten durchführen kann, erlangt ein Unternehmen Wettbewerbsvorteile.

\begin{tabular}{|c|c|c|c|c|}
\hline \multicolumn{5}{|c|}{ Unternehmungsinfrastruktur } \\
\hline \multicolumn{5}{|c|}{ Personalpolitik } \\
\hline \multicolumn{5}{|c|}{ Technologieentwicklung } \\
\hline \multicolumn{5}{|c|}{ Beschaffungspolitik } \\
\hline $\begin{array}{l}\text { Forschung } \\
\text { und } \\
\text { Entwicklung }\end{array}$ & $\begin{array}{l}\text { Beschaffungs- } \\
\text { logistik }\end{array}$ & Produktion & Design & $\begin{array}{l}\text { Marketing/ } \\
\text { Vertrieb }\end{array}$ \\
\hline $\begin{array}{l}\text { - Kooperative } \\
\text { F\&E } \\
\text { - Auftrags- } \\
\text { F\&E } \\
\text { - Kooperation } \\
\text { mit wiss. } \\
\text { Instituten } \\
\text { - Lizenzen } \\
\text { - Patente } \\
\text { - Know-how }\end{array}$ & $\begin{array}{l}\text { - Zentraler } \\
\text { Einkauf } \\
\text { - De-Vertika- } \\
\text { lisierung } \\
\text { - Integration } \\
\text { - Lieferanten- } \\
\text { koordination }\end{array}$ & $\begin{array}{l}\text { - Eigenfertigung } \\
\text { vs. Fremd- } \\
\text { bezug } \\
\text { - Kapazitäten } \\
\text { - Standorte } \\
\text { - Automati- } \\
\text { sierung } \\
\text { - Standardi- } \\
\text { sierung } \\
\text { - Durchlauf- } \\
\text { zeiten } \\
\text { - Liefertreue } \\
\text { - Qualitäts- } \\
\text { sicherung }\end{array}$ & $\begin{array}{l}\text { - Funktion } \\
\text { - Ästhetik } \\
\text { - Qualität }\end{array}$ & $\begin{array}{l}\text { - Marketing- } \\
\text { Mix } \\
\text { - Messe- } \\
\text { präsenz } \\
\text { - Garantie } \\
\text { - Kunden- } \\
\text { dienst } \\
\text { - Schnellig- } \\
\text { keit } \\
\text { - Organisation }\end{array}$ \\
\hline$L$ & 1 & 1 & $\perp$ & - \\
\hline
\end{tabular}

Abbildung 15: Die Wertkette 197

195 Vgl. PORTER, 1999 (b), S. 68.

196 PORTER, 1999 (b), S. 63.

197 Vgl. HINTERHUBER, 1996 (a), S. 182, in Anlehnung an: PORTER, 1999 (b), S. 66. 
In Abbildung 15 stellt HINTERHUBER die Wertkette in Anlehnung an PORTER dar. Im unteren Teil dieser Abbildung weist HINTERHUBER auf den Konkurrentenvergleich hin und nennt einige konkrete Ansatzpunkte, die dabei in Betracht gezogen werden können.

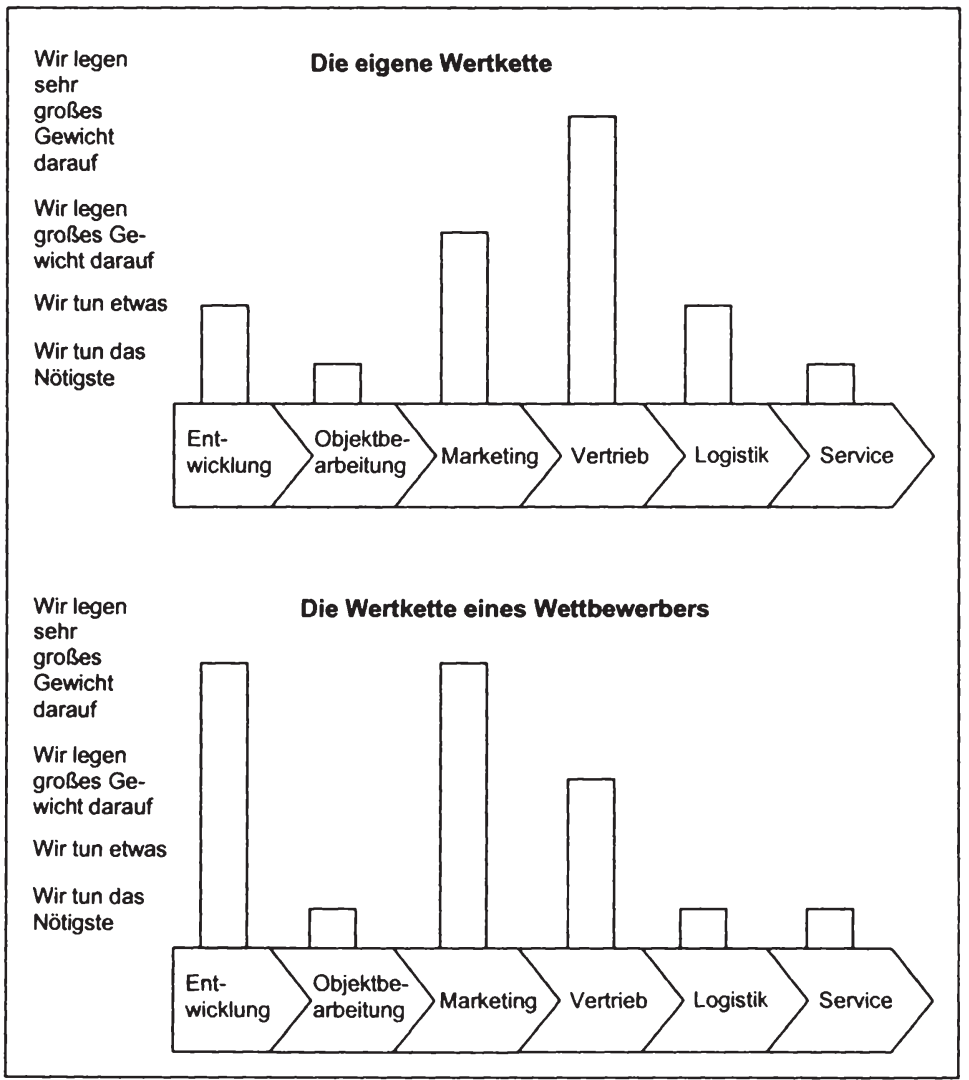

Abbildung 16: Der Wertkettenvergleich in der Konkurrenzanalyse ${ }^{198}$

Der Vergleich der eigenen Wertkette mit denen von Konkurrenten liefert Hinweise darüber, „durch welche Schwerpunkte bei den Wertaktivitäten ein Wettbewerbsvorteil erreicht werden kann"199. Die Rekonstruktion der Wertketten von Konkurrenzunternehmen unterscheidet sich nicht von der Vorgehensweise bei

198 Vgl. ESSER, 1989, S. 203.

199 WELGE/AL-LAHAM, 1992, S. 125, in Anlehnung an: ESSER, 1989, S. $205 \mathrm{ff}$. 
der Erstellung der eigenen Wertkette. Dabei soll auf grobe Schätzungen zurückgegriffen werden, falls quantitative Informationen über die Wertaktivitäten der Wettbewerber nicht ermittelbar sind. 200

ESSER zeigt in Abbildung 16 ein Beispiel für den Vergleich von Wertketten im Rahmen einer konkurrenzanalytischen Betrachtung. Dabei werden nur Schwerpunkte, d.h. Wertaktivitäten untersucht, die in einer bestimmten Branche oder in einer individuellen Situation von Interesse sind. Außerdem wird die Wertigkeit abgeschätzt, die der jeweiligen Aktivität im eigenen und im Konkurrenzunternehmen zukommt. Diese Wertigkeit wird, wie in Abbildung 16 zu sehen ist, nur in vier grobe Stufen eingeteilt. In vielen Fällen wird diese grobe Einteilung von Branchenkennern ohne detaillierte quantitative Informationen vorgenommen werden können, so dass sich ein Gesamtbild von wichtigen Wertaktivitäten und deren ungefähre Bedeutung im jeweiligen Unternehmen ergibt.

Nicht nur die Kenntnis von Schwerpunkt-Aktivitäten und deren unternehmensinterne Bedeutung kann das Ergebnis einer Wertkettenanalyse sein. Durch die Zerlegung in einzelne Wertaktivitäten wird eine komplexe Organisation für konkurrierende Unternehmen - auch bezüglich der Kostensituation - transparenter. Aus diesem Grund bezeichnet PORTER die Wertkette als das „Grundinstrument zur Bestimmung der relativen Kosten der Konkurrenten“201.

Ein Beispiel aus der Möbelbranche, in dem besonders die Kostenlage analysiert wird, stellt ESSER in Tabelle 2 dar.

Obwohl sich die Kosten von Wettbewerbern oft nur annäherungsweise bestimmen lassen, erhält man doch Kostendifferenzen zwischen den Wettbewerbern, die „ein Bild der relativen Kostenposition eines Konkurrenten"202 erkennen lassen.

$200 \mathrm{VgI.} \mathrm{WELGE/AL-LAHAM,} \mathrm{1999,} \mathrm{S.} 275$.

201 PORTER, 1999 (b), S. 142.

202 PORTER, 1999 (b), S. 142. 


\begin{tabular}{|l|l|l|l|l|l|l|l|}
\cline { 2 - 7 } \multicolumn{1}{c|}{} & $\begin{array}{l}\text { Roh- } \\
\text { material }\end{array}$ & $\begin{array}{l}\text { Her- } \\
\text { stellung }\end{array}$ & Montage & $\begin{array}{l}\text { Trans- } \\
\text { port }\end{array}$ & $\begin{array}{l}\text { Show- } \\
\text { room }\end{array}$ & $\begin{array}{l}\text { Liefer- } \\
\text { zeit }\end{array}$ & $\begin{array}{l}\text { Anlie- } \\
\text { ferung }\end{array}$ \\
\hline $\begin{array}{l}\text { Herkömm- } \\
\text { liche Mö- } \\
\text { belan- } \\
\text { bieter }\end{array}$ & $\begin{array}{l}\text { Je nach } \\
\text { Material: } \\
\text { geringe } \\
\text { bis hohe } \\
\text { Kosten }\end{array}$ & $\begin{array}{l}\text { Kleine } \\
\text { Mengen: } \\
\text { hohe } \\
\text { Kosten }\end{array}$ & $\begin{array}{l}\text { Arbeits- } \\
\text { intensiv: } \\
\text { hohe } \\
\text { Kosten }\end{array}$ & $\begin{array}{l}\text { Luft: } \\
\text { hohe } \\
\text { Kosten }\end{array}$ & $\begin{array}{l}\text { Zentrale } \\
\text { Lage: } \\
\text { hohe } \\
\text { Kosten }\end{array}$ & $\begin{array}{l}\text { Kleines } \\
\text { Lager: } \\
\text { lang }\end{array}$ & $\begin{array}{l}\text { Luft: } \\
\text { Hohe } \\
\text { Kosten }\end{array}$ \\
\hline $\begin{array}{l}\text { IKEA } \\
\text { Keringe }\end{array}$ & $\begin{array}{l}\text { Große } \\
\text { Mengen: } \\
\text { geringe } \\
\text { Kosten }\end{array}$ & $\begin{array}{l}\text { Durch } \\
\text { Kunden: } \\
\text { keine } \\
\text { Kosten }\end{array}$ & $\begin{array}{l}\text { Zerleg- } \\
\text { bar: } \\
\text { geringe } \\
\text { Kosten }\end{array}$ & $\begin{array}{l}\text { Außer- } \\
\text { halb: } \\
\text { geringe } \\
\text { Kosten }\end{array}$ & $\begin{array}{l}\text { Großes } \\
\text { Lager: } \\
\text { kurz }\end{array}$ & $\begin{array}{l}\text { Durch } \\
\text { Kunden: } \\
\text { keine } \\
\text { Kosten }\end{array}$ \\
\hline
\end{tabular}

Tabelle 2: Die Bestimmung relativer Kosten mit Hilfe der Wertkette ${ }^{203}$

Steht die Kostenanalyse im Mittelpunkt der Betrachtung, sind drei Grundsätze bei der Aufgliederung in einzelne Wertaktivitäten zu berücksichtigen:204

1. Höhe und Wachstum der Kosten einer Aktivität,

2. Kostenverhalten einer Aktivität und

3. Unterschiede bei der Ausführung der Aktivität zwischen den Konkurrenten.

Zur Analyse der Kostenstruktur werden den Wertaktivitäten „Betriebskosten“ und „Anlagegüter" zugeordnet.205 Das Ziel ist es, einen Überblick über die Kostenverteilung im Unternehmen nach Wertaktivitäten zur Aufhellung strategischer Kostenfragen zu erhalten. „Ein Kostenvorsprung ergibt sich, wenn das Unternehmen bei der Durchführung seiner Wertaktivitäten niedrigere Gesamtkosten als seine Konkurrenten erreicht." ${ }^{206}$ Das führt zu der Frage nach dem Verhalten und den Antriebskräften der Kosten.

Unter Kostenantriebskräften versteht PORTER eine Reihe „kostenwirksamer Strukturfaktoren"207, die das Kostenverhalten einer Wertaktivität - und damit die Kostenposition eines Unternehmens - beeinflussen. Sie sind die strukturbedingten Ursachen der Kosten einer Aktivität.

203 Vgl. ESSER, 1994, S. 137.

204 Vgl. PORTER, 1999 (b), S. 100.

205 Ausführlicher vgl. PORTER, 1999 (b), S. $101 \mathrm{ff}$.

206 PORTER, 1999 (b), S. 99.

207 PORTER, 1999 (b), S. 106. 
PORTER erläutert die einzelnen Kostenantriebskräfte ausführlich, die jedoch in diesem Rahmen nur exemplarisch wiedergegeben werden sollen:208

- Eine betriebsgrößenbedingte Kostendegression ergibt sich bspw., „wenn ein Unternehmen mit größerem Geschäftsvolumen Tätigkeiten anders oder rationeller ausführen oder den Kostenaufwand für immaterielle Anlagewerte, wie Werbung, F\&E, über ein größeres Absatzvolumen amortisieren kann“209.

- Lernvorgänge im Sinne von Know-how- und Verfahrensverbesserungen können die Kosten einer Aktivität mehr oder weniger günstig beeinflussen. Gefördert wird dieser Prozess durch Lieferanten, Unternehmensberater oder bspw. durch ein Reverse Engineering. ${ }^{210}$

- Strukturell kostenbeeinflussend wirkt sich der Standort aus, denn das Lohnniveau, Kosten für Rohstoffe und Energie oder die Steuersätze sind regional verschieden.

Die Kosten einer Aktivität werden meist durch ein Zusammenspiel mehrerer Kostenantriebskräfte hervorgerufen, die sich gegenseitig verstärken oder neutralisieren.

„Die relative Kostenposition eines Unternehmens ist eine Funktion der Zusammensetzung seiner Wertkette im Vergleich zu der der Konkurrenz und seiner relativen Position in Bezug auf die Kostenantriebskräfte jeder Wertaktivität." 211

Den Zusammenhang zwischen der Wertkette, den Kostenantriebskräften und der Kostenposition nutzt PORTER für die Bestimmung der relativen Kosten der Konkurrenten. ${ }^{212}$ Dabei geht es darum, deren Kosten annäherungsweise zu schätzen, was ausreicht, um daraus Rückschlüsse auf die eigene strategische Position ziehen zu können.

Die Wertkette ist, wie gezeigt wurde, ein Instrument, mit dem eine Kostenbetrachtung von Wettbewerbern im Rahmen der Konkurrenzanalyse durchgeführt werden kann. Durch die Aufteilung eines Unternehmens in mehrere strategisch

\footnotetext{
208 Ausführlicher vgl. PORTER, 1999 (b), S. $106 \mathrm{ff}$.

209 PORTER, 1999 (b), S. 107.

210 Vgl. PORTER, 1999 (b), S. $110 \mathrm{f}$.

211 PORTER, 1999 (b), S. 141.

212 Vgl. PORTER, 1999 (b), S. $142 \mathrm{f}$.
} 
bedeutsame Aktivitäten wird eine komplexe Organisation übersichtlicher, durchsichtiger, und Schwerpunkte sind leichter erkennbar. Bei der Kostenanalyse ermöglicht die größere Transparenz die Suche nach den Kostenantriebskräften einzelner Aktivitäten, die in der Summe die Gesamtkostenposition des eigenen wie des Konkurrenzunternehmens bestimmen.

\subsubsection{Portfolio-Analyse in der Konkurrenzanalyse}

Die Portfolio-Analyse ist vor allem ein Instrument der strategischen Analyse und nur in zweiter Linie ein Instrument zur Formulierung von Strategien. ${ }^{213}$ Allerdings fließen die Ergebnisse der Analyse in die Strategieformulierung, so dass man meist folgende Zielsetzungen mit der Portfolio-Analyse verfolgt:214

- Über den Mittelzufluss und die Ressourcenverteilung sollen die Geschäftsbereiche koordiniert und strategisch gesteuert werden.

- Durch ein Gleichgewicht zwischen mittelbindenden und mittelfreisetzenden sowie risikoreichen und risikoarmen Geschäftsbereichen soll ein interner Finanz- und Risikoausgleich erfolgen.

- Die Analyse hilft bei der Portfolio-Optimierung, d.h. Stärkung der Kerngeschäfte, Rückzug aus Randgeschäften und Aufbau von Neugeschäften, die den Kerngeschäften verwandt sind. ${ }^{215}$

Das Konzept der Portfolio-Analyse basiert auf einer „Verdichtung und Selektion unternehmungsbezogener Erfolgsfaktoren auf einer Unternehmungsdimension und umweltbezogener Erfolgsfaktoren auf einer Umweltdimension“216.

Die Portfolio-Analyse lehnt sich damit an die bereits erläuterte Umwelt- und Unternehmensanalyse an, die Chancen und Risiken sowie Stärken und Schwächen identifiziert. Die Portfolio-Analyse geht darüber hinaus und liefert zusätzliche Erkenntnisse, die im Rahmen der Konkurrenzanalyse von Interesse sein können und ist damit die höchste Verdichtungsstufe im Konkurrenzvergleich. ${ }^{217}$

\footnotetext{
213 Vgl. HAMMER, 1995, S. $183 \mathrm{f}$.

$214 \mathrm{Vgl}$. Welge/AL-LAHAM, 1999, S. 324.

215 Vgl. TiMmermanN, 1988, S. 99.

216 HAMMER, 1995, S. 179.

217 Vgl. HoffmanN, 1986, S. $201 \mathrm{ff}$.
} 
Unter einem Portfolio versteht HINTERHUBER "die Gesamtheit der Produkte und/oder Dienstleistungen, mit denen sich die Unternehmung zu einem gegebenen Zeitpunkt auf dem Markt präsentiert"218. Strategische Geschäftseinheiten eines Unternehmens, synonym wird der Begriff Geschäftsbereich verwendet, können in einer Portfolio-Matrix dargestellt und analysiert werden. Unter einer Strategischen Geschäftseinheit wird ein eigenständiges Aktivitätenfeld der Unternehmung im Sinne einer Produkt-Markt-Technologie-Kombination verstanden, das als Ganzes Gegenstand strategischer Entscheidungen ist. ${ }^{219}$ Die Visualisierung der segmentierten Unternehmungsaktivitäten ergibt ein Bild, das für das Gesamtunternehmen

- die gegenwärtige und zukünftige Erfolgsträchtigkeit einzelner Geschäftsbereiche darstellt,

- auf gegenwärtige oder zukünftige finanzielle (Un-) Gleichgewichte hinweist,

- Stärken und Schwächen einzelner Geschäftsbereiche gegenüber Konkurrenten aufdeckt und

- Hinweise für die Formulierung strategischer Ziele liefert, indem Normstrategien vorgeschlagen werden. 220

Im Hinblick auf ihre hohe konkurrenzanalytische Aussagekraft wird im Folgenden die Marktattraktivitäts-Geschäftsfeldstärken-Matrix ausführlich dargestellt, die in den 70er-Jahren von der General ELECTRIC COMPANY und der Beratungsgesellschaft MCKINSEY \& COMPANY entwickelt wurde.

Dieser Portfolio-Ansatz, synonym wird in der Literatur auch die Bezeichnung Branchenattraktivität-Wettbewerbsstärke-Matrix verwendet, besteht aus einem Raster, dessen Ordinate die Marktattraktivität darstellt. Auf der Abszisse werden die Wettbewerbsstärke, bzw. die relativen Wettbewerbsvorteile der Unternehmung abgetragen, wie in Abbildung $17 \mathrm{zu}$ sehen sein wird. 221

Die Markt- bzw. Branchenattraktivität, als Umweltdimension, spiegelt sich in den Gewinn- und Wachstumsperspektiven der entsprechenden Branche wider. Sie

218 HINTERHUBER, 1996 (a), S. 148.

$219 \mathrm{Vgl}$. GÄLWEILER, 1979, S. 259.

$220 \mathrm{Vgl}$. HAMMER, 1995, S. 179.

221 Vgl. Welge/AL-LAHAM, 1999, S. 343 ff.; HAX/MAJLuF, 1991, S. $180 \mathrm{ff}$. 
können anhand von vier Hauptkriterien, die vom einzelnen Unternehmen nicht beeinflussbar sind, beurteilt werden:222

1. Marktwachstum und Marktgröße,

2. Marktqualität, gemeint ist die Gewinnstabilität der Branche,

3. Versorgung mit Energie, Rohstoffen, Komponenten, Halbfertigfabrikaten und

4. Umweltsituation im Sinne von Unternehmungsumfeld.

Die Geschäftsfeld- bzw. Wettbewerbsstärke stellt die Unternehmungsdimension dar. Die Wettbewerbsstärke wird bestimmt durch den Vergleich mit dem stärksten Konkurrenzunternehmen, wodurch sich relative Wettbewerbsvorteile ergeben. HINTERHUBER nennt dafür u.a. folgende Kriterien:223

1. Die relative Marktposition, die aus folgenden fünf Kräften resultiert, die gegenwarts- oder zukunftsbezogen betrachtet werden können:

a) Absoluter und relativer Marktanteil zum stärksten Konkurrenten und seine Entwicklung innerhalb des Planungshorizontes,

b) Rentabilität: Deckungsbeiträge, Umsatzrendite und Kapitalumschlag,

c) Risiko, d.h. der Grad der Etabliertheit am Markt, Kosten- und Preisentwicklung, Vorschriftenänderungen,

d) Marketingpotential: Image der Unternehmung und daraus resultierende Abnehmerbeziehungen, Preisvorteil aufgrund von Qualität, Lieferzeiten, Service, usw., Kontrollierbarkeit der Vertriebswege, etc.,

e) Art der Quellen von Wettbewerbsvorteilen: Differenzierung und/oder Kostenführerschaft.

2. Das relative Produktionspotential, das HINTERHUBER ansieht als eine „Kombination aus Prozesswirtschaftlichkeit, Stand der Hardware, Standort und Anzahl der Fertigungsbetriebe sowie Zugang zu Rohstoffen, Komponenten und Vormaterialien“224. Folgende Aspekte sind dabei zu berücksichtigen:

a) Prozesswirtschaftlichkeit: Kostenvorteile gegenüber Konkurrenten aufgrund moderner Produktionsverfahren, Produktionsbedingungen, Kapazitätsausnutzung, Größe der Produktionseinheiten, Einbindung in strategische Netzwerke, etc..

222 Vgl. HiNTERHUBER, 1996 (a), S. 150.

$223 \mathrm{Vgl}$. HINTERHUBER, 1996 (a), S. $151 \mathrm{ff}$.

224 HINTERHUBER, 1996 (a), S. 152. 
b) Hardware: Standortvorteile, Steigerungspotential der Produktivität, etc..

c) Zugang zu Rohstoffen, Energieträgern und Halbfabrikaten: Kostensituation der Energie- und Rohstoffversorgung im Vergleich zu den wichtigsten Konkurrenten, etc..

3. Das relative Forschungs- und Entwicklungspotential als eine „Kombination aus Produktpalette, Produktqualität, Innovationsrate, Anwendungstechnik und technischer Assistenz"225. Das relative Forschungs- und Entwicklungspotential beschreibt das technische Leistungspotential der Unternehmung.

4. Die relative Qualifikation der Führungskräfte und Mitarbeiter, bewertet durch die Professionalität, die Urteilsfähigkeit, den Einsatz und die Kultur der Mitarbeiter, das Innovationsklima, die Qualität der Führungssysteme, etc..

5. Die Kernkompetenzen der Unternehmung.

Mit Hilfe der genannten Kriterien lassen sich Geschäftsbereiche auf der Achse der Wettbewerbsstärke in Relation zu Konkurrenzunternehmen darstellen.

Neben der Analyse eigener Strategischer Geschäftseinheiten im Vergleich zu Wettbewerbern bietet die Portfolio-Methodik die Möglichkeit, Gesamtportfolios führender Wettbewerber darzustellen, um die strategischen Möglichkeiten abzuschätzen und das zweckmäßigste Wettbewerbsverhalten festzulegen.226 Abbildung 17 zeigt ein beispielhaftes Schema der Konkurrenzanalyse mit Hilfe der Portfolio-Analyse nach MCKINSEY \& COMPANY.

In Kombination mit dem bereits erläuterten System zur Konkurrenzanalyse von PORTER entwickelt HINTERHUBER ein "qualitatives Bild"227 der Konkurrenzsituation, das es ermöglicht, wahrscheinliche zukünftige strategische Züge von Wettbewerbern zu erkennen, sofern diese der „ökonomischen Logik"228 folgen.229 Durch das bessere Verständnis der strategischen Situation von Wettbewerbern ergänzt die Portfolio-Methodik damit die Bestimmung des Reaktionsprofils von Konkurrenten, wie in Abbildung $17 \mathrm{zu}$ sehen ist.

\footnotetext{
225 HINTERHUBER, 1996 (a), S. 152.

226 Vgl. HINTERHUBER, 1996 (a), S. 171.

227 HINTERHUBER, 1996 (a), S. 171.

228 HINTERHUBER, 1996 (a), S. 173.

229 Ähnlich: HAX/MAJluf, 1991, S. 39.
} 


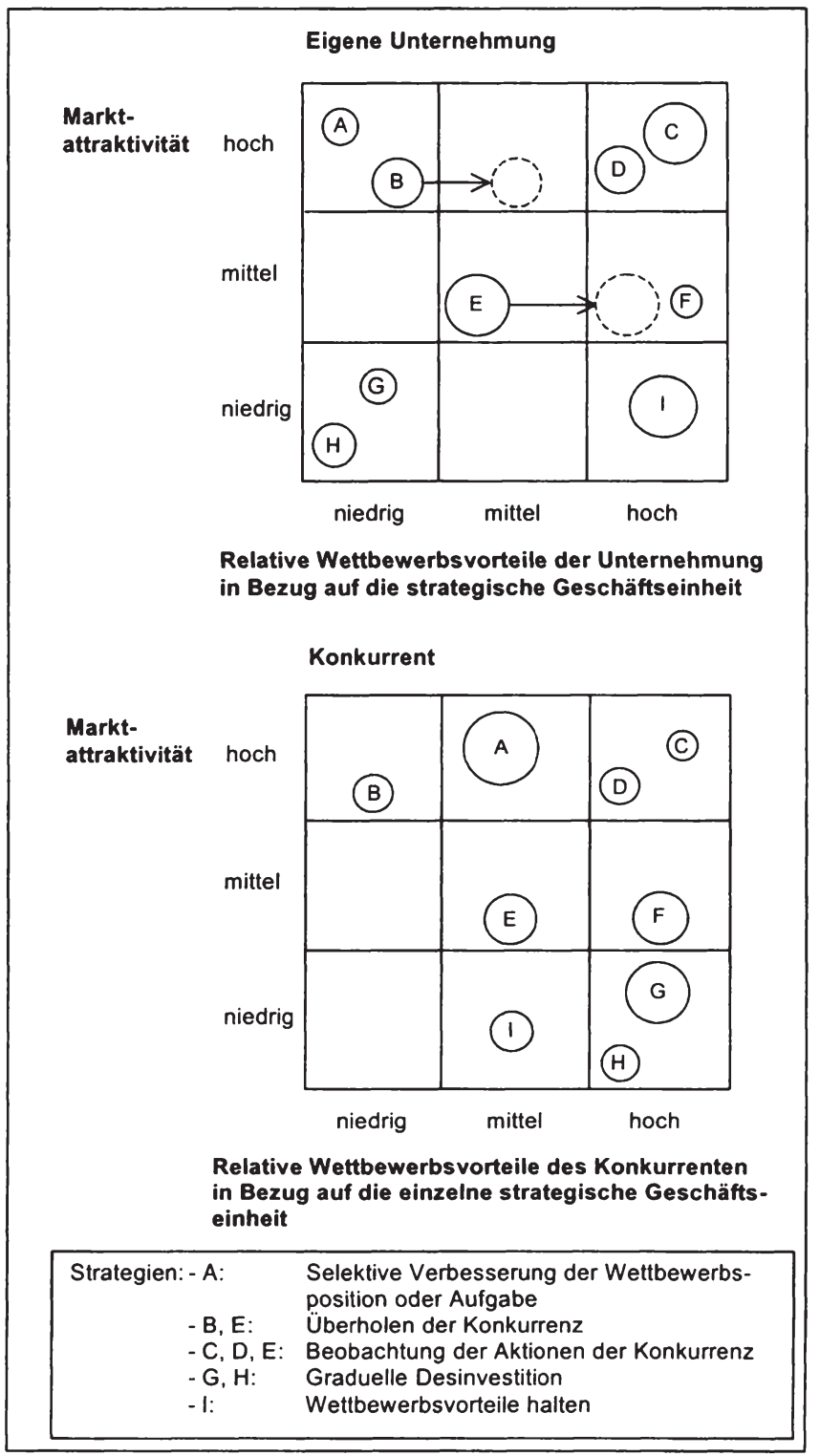

Abbildung 17: Die Portfolio-Analyse von Wettbewerbern ${ }^{230}$ 
Der Portfolio-Ansatz wurde in diesem Abschnitt als strategisches AnalyseInstrument vorgestellt.231 Obwohl die Portfolio-Analyse in der Literatur nicht als Instrument der Konkurrenzanalyse ausgewiesen wird, hat sich gezeigt, dass die Informationen, die eine Portfolio-Betrachtung liefert, sehr wohl für diesen Zweck verwendet werden können.

In diesem 3. Kapitel wurde erläutert, welche Inhalte mit Hilfe bestimmter Instrumente erfassbar sind und welcher Nutzen damit für die Konkurrenzanalyse erzielt werden kann. Bei einigen Instrumenten wurden bereits Beispiele angesprochen, inwiefern Kosten von Konkurrenzunternehmen betrachtet werden können. Die Beschaffung dieser Informationen wird im folgenden 4. Kapitel genauer untersucht.

231 Die im Rahmen der vorliegenden Untersuchung nicht erörterten Aspekte des Portfolio-Ansatzes, wie bspw. die eigentliche Erstellung einer Portfolio-Matrix, die Positionierung der Geschäftsbereiche und die Ableitung bestimmter Normstrategien, werden in der einschlägigen Literatur detailliert vorgestellt. Eine ausführliche Darstellung findet sich bspw. bei: HINTERHUBER, 1996 (a), S. 154 ff. und bei: WELGE/AL-LAHAM, 1999, S. $324 \mathrm{ff}$. 


\section{Kapitel Informationsgewinnung in der Konkurrenzanalyse}

Informationen über Wettbewerber sind der „Rohstoff“ jeder Konkurrenzanalyse. Die Gewinnung von Informationen über Konkurrenzunternehmen stellt damit eine grundlegende Aufgabe dar, ohne die das Wettbewerbsumfeld eines Unternehmens im Rahmen der strategischen Planung nur unvollständig abgebildet und in die strategischen Überlegungen einbezogen würde. In diesem Kapitel sollen, nach der grundlegenden Klärungen des Begriffs "Information" und der Darstellung des betrieblichen Informationssystems, im zweiten Abschnitt die für die Konkurrenzanalyse relevanten Informationsquellen aufgezeigt werden. Im dritten Abschnitt steht speziell die Gewinnung von Kosteninformationen von Wettbewerbsunternehmen im Vordergrund.

\subsubsection{Begriff der Information und Darstellung des betrieblichen Informationssystems}

Dem Begriff der Information kommt in der vorliegenden Arbeit, besonders aber im 4. Kapitel, eine herausragende Bedeutung zu. Der im Allgemeinen in der betriebswirtschaftlichen Literatur verwendete Informationsbegriff geht auf WITTMANN zurück, der Information als „zweckorientiertes Wissen“232 bezeichnet. Zweckorientiert ist Wissen dann, wenn es für ein bestimmtes Handeln oder zur Zielerreichung benötigt wird. Das „Ausgangsmaterial“ von Informationen sind Daten. Diese entsprechen erfassten, aber unverarbeiteten Nachrichten und müssen aufgaben- oder verwendungsorientiert sein, damit sie zu Informationen werden.

Den Terminus Wissen begrenzt WITTMANN nicht nur auf den strengen Sinn der Gewissheit, sondern erweitert inn um das wahrscheinliche Wissen. Daraus folgt, dass zweckorientiertes Wissen nicht nur vergangenheitsgerichtet sein muss, sondern auch ungewiss und somit zukunftsbezogen sein kann. Das Gleiche gilt folglich für die Information. ${ }^{233}$

Wenn Informationen für eine klar definierbare Bestimmung benötigt werden, besteht ein Bedarf an Informationen. Um diesen zu ermitteln, muss die Frage

232 WitTMANN, 1956, S. 14.

233 Vgl. WittManN, 1956, S. $13 \mathrm{ff}$. 
beantwortet werden, welches Wissen bzw. welche Informationen für diesen bestimmten Zweck notwendig oder relevant sind, wobei der objektive und der subjektive Informationsbedarf divergieren können.234 Dazu stellt HEINEN allgemein fest, dass Informationen dann relevant sind, „wenn sie aus der Sicht eines Entscheidungsträgers zur Erfassung und Lösung eines Problems beitragen"235.

Da sehr unterschiedliche Informationen in einem Unternehmen regelmäßig beschafft werden, ist ein Konkurrenzinformationssystem als ein Teil eines gesamten betrieblichen Informationssystems zu sehen. Ein Konkurrenzinformationssystem umfasst „die Summe aller Aktivitäten, die der Erhebung, Aufbereitung, Speicherung und Verteilung von Konkurrenzinformationen dienen" ${ }^{236}$. Ein funktionsfähiges betriebliches Informationssystem ist eine grundlegende Voraussetzung für eine umfassende Informationsversorgung der einzelnen Funktionsbereiche des Unternehmens. ${ }^{237}$ Innerhalb des betrieblichen Informationssystems kann zwischen unternehmensinternen und -externen Informationen unterschieden werden, so dass man die in Abbildung 18 gezeigte Strukturierung vornehmen kann.

Während einzelne Bereiche des betrieblichen Informationssystems nur von einem Teil der Mitarbeiter betreut werden, wie bspw. das Rechnungswesen oder der Personalbereich, sollte die Beschaffung von Konkurrenzinformationen auf den Beobachtungen möglichst vieler Mitarbeiter basieren.238 Eine große Anzahl an Beobachtern aus unterschiedlichen Funktionsbereichen des Unternehmens mit einem entsprechend heterogenen Bildungs- und Erfahrungshintergrund gewährleistet eine stabile Grundlage der Informationsgewinnung, da der Informationsfluss über eine entsprechend große Anzahl an Kanälen läuft. Zusätzlich wird eine differenzierte Betrachtung ermöglicht, die aus unterschiedlichen Blickwinkeln erfolgen kann. Voraussetzung dafür ist jedoch eine entsprechende Motivation der Mitarbeiter, Konkurrenzinformationen zu liefern.

\footnotetext{
$234 \mathrm{Vgl}$. HEINEN, 1991, S. 275 f.

235 HEINEN, 1983, S. 1040.

236 LINK, 1988, S. $136 \mathrm{f}$.

$237 \mathrm{Vgl}$. LINK, 1988, S. 136. HOFFMANN und NAGEL stufen ein funktionsfähiges Informationssystem sogar als einen wesentlichen Erfolgsfaktor eines Unternehmens ein. Vgl. HOFFMANN, 1986, S. 835; NAGEL, 1986, S. 55 und S. 141.
}

$238 \mathrm{Vgl}$. FISCHER, 1986, S. 114. 


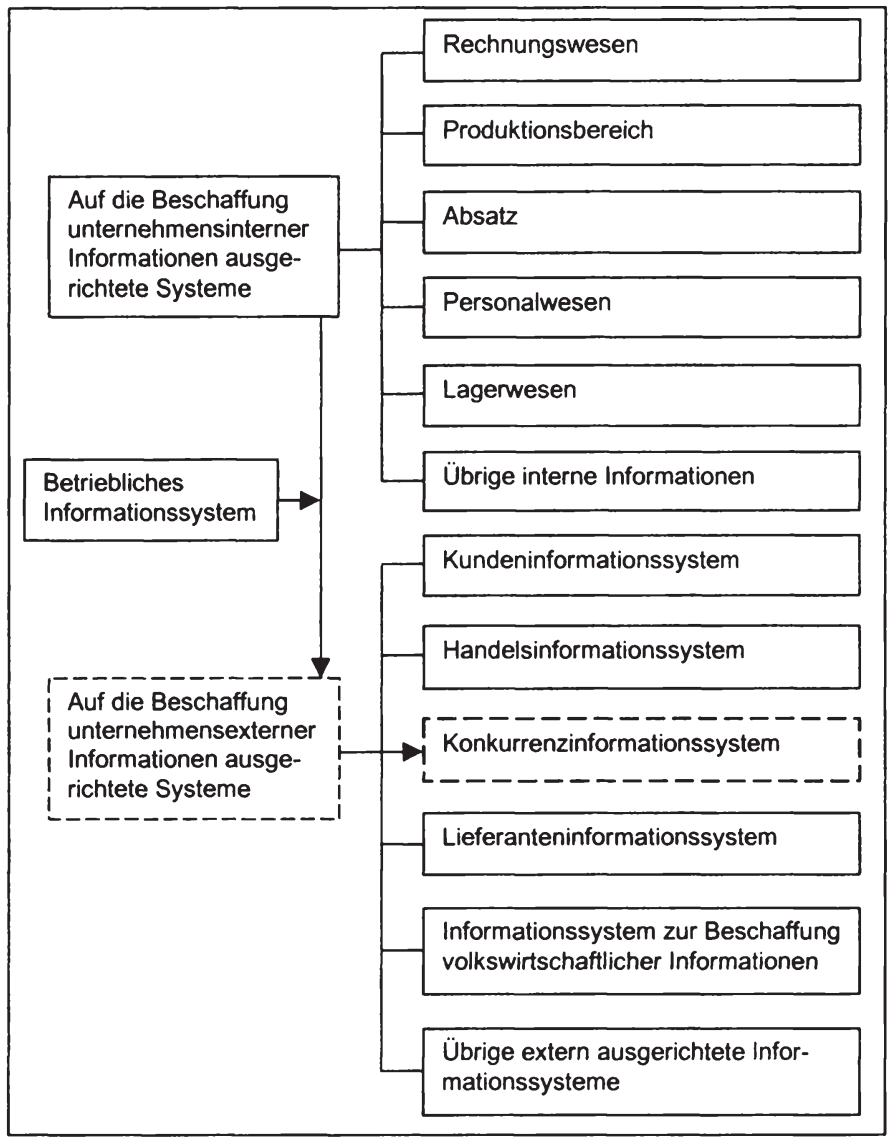

Abbildung 18: Das betriebliche Informationssystem 239

Die Vielzahl und die Komplexität der für eine umfassende Konkurrenzanalyse notwendigen Informationen erfordern eine systematische und institutionalisierte Informationsbeschaffung, damit unternehmenspolitische Entscheidungen nicht auf der Basis zufällig vorhandener Daten und Meinungen gefällt werden. ${ }^{240} \mathrm{Da}$ viele Informationen nicht beliebig zur Verfügung stehen und jederzeit abrufbereit sind, sondern - ähnlich einem Mosaik - aus diversen unterschiedlichen 
Quellen bruchstückhaft zu einem Gesamtbild zusammengefügt werden, ist die Informationsgewinnung kontinuierlich und über einen längeren Zeitraum anzulegen.241 Darüber hinaus müssen eindeutige Zuständigkeiten geschaffen werden, die den Prozess des Sammelns und der Bündelung der Einzelinformationen organisatorisch regeln.

\section{I.4.2 Informationsquellen in der Konkurrenzanalyse}

Man unterscheidet zwischen Primär- und Sekundärquellen, die jeweils zu verschiedenen Arten der Durchführung der Informationsbeschaffung führen.242 Die Primärforschung basiert auf neuem, konkret für einen bestimmten Zweck, bzw. für eine benötigte Erkenntnis erhobenem Datenmaterial. Dagegen stützt sich die Sekundärforschung ausschließlich auf vorhandene Daten, die zu einem früheren Zeitpunkt, oft für einen anderen Zweck erfasst wurden und die einer Zweit- oder Drittauswertung unterzogen werden.243 Gegenüber der Primärforschung, deren Vorteile besonders in der Aktualität und in der zielgenauen Informationsgewinnung liegen, ist die Sekundärforschung meist kostengünstiger und schneller, da das Datenmaterial bereits vorliegt und nicht erst erhoben werden muss. Demnach bietet es sich im Rahmen der Konkurrenzanalyse an, in einem ersten Schritt auf Sekundärmaterial zurückzugreifen, um danach zielgerichtet Primärquellen als Ergänzung oder Überprüfung zu nutzen. ${ }^{244}$

Informationen und deren Quellen müssen bestimmte Anforderungen erfüllen, die im Folgenden erläutert werden. ${ }^{245}$

1. Verfügbarkeit: Konkurrenzbezogenes Datenmaterial ist die Basis und damit die notwendige Voraussetzung jeder Konkurrenzanalyse. Es sollte möglichst regelmäßig über die jeweilige Quelle bezogen werden können. Damit steigt die Vergleichbarkeit der Daten, sowie deren Zuverlässigkeit.

2. Zuverlässigkeit: Die Qualität des Datenmaterials muss gewährleistet sein. Dies entspricht der Forderung nach Authentizität, also Echtheit und Glaub-

\footnotetext{
241 Vgl. PORTER, 1999 (a), S. $114 \mathrm{ff}$.

242 Vgl. Berekoven/ECKERT/EllenRieder, 1996, S. 42 und S. 49; LinK, 1988, S. $140 \mathrm{f}$.

243 Vgl. ROGGE, 1981, S. 49.

244 Vgl. JOAS, 1990, S. 158 ff.; LINK, 1988, S. $140 \mathrm{ff}$.

245 In Anlehnung an: LINK, 1988, S. $141 \mathrm{ff}$.
} 
würdigkeit der gewonnenen Daten. Informationsquellen sollten möglichst überprüfbar und nachvollziehbar sein. Da falsche Informationen eine Gefahr darstellen, ist die Zuverlässigkeit einer Quelle von hoher Bedeutung.

3. Kostengünstigkeit: Die Kosten der Informationsgewinnung und der Nutzen, den diese Informationen aufweisen, müssen in einem ökonomisch sinnvollen Verhältnis zueinander stehen. Sekundärdaten sind im Allgemeinen kostengünstiger, als die Erhebung von Primärdaten.

4. Aktualität: Die Informationsquelle muss Wettbewerbsinformationen zeitnah, d.h. bei aktuellen Problemstellungen rechtzeitig zur Verfügung stellen. Sekundärmaterial hat diesbezüglich den Vorteil, dass es bereits vorhanden ist und nur einer neuen Auswertung zugeführt werden muss, hat jedoch gleichzeitig den Nachteil, dass es auf älteren Daten basiert als Primärmaterial. Gerade Sekundärdaten sind folglich auf ihre Aktualität hin zu überprüfen.

Unter Beachtung des Anforderungskataloges kann ein Unternehmen für die Konkurrenzanalyse auf zahlreiche Informationsquellen zugreifen. Abbildung 19 gliedert eine Auswahl wichtiger Quellen nach Primär- und Sekundärquellen, sowie nach unternehmensinternen und -externen Informationsquellen.

Die Vielzahl genannter Informationsquellen ermöglicht eine Betrachtung der relevanten Wettbewerber ${ }^{246}$ aus vielen unterschiedlichen Perspektiven, so dass sich durch eine systematische Analyse ein Gesamtbild ergibt. Die für die Konkurrenzanalyse tatsächlich im Einzelfall verwendbaren Quellen sind abhängig von den spezifischen Branchenverhältnissen. Allgemein können jedoch die Mitglieder der Geschäfts- und Vertriebsleitung, der Außendienst, Veröffentlichungen der Wettbewerber, die Kunden, Presseberichte, ehemalige Mitarbeiter von Wettbewerbern und die Konkurrenzprodukte selber als wesentliche Quellen von Wettbewerbsinformationen hervorgehoben werden. ${ }^{247}$

246 Der Begriff der „relevanten Wettbewerber" wird von HAX/MAJLUF verwendet und im 2. Kapitel der vorliegenden Arbeit erläutert. Vgl. HAX/MAJLUF, 1991, S. $338 \mathrm{f}$.

247 in Anlehnung an: LINK, 1988, S. 148. 


\begin{tabular}{|c|c|c|}
\hline & Primärquellen & Sekundärquellen \\
\hline $\begin{array}{l}\text { Unternehmens- } \\
\text { interne Quellen }\end{array}$ & $\begin{array}{l}\text { - } \quad \text { Marktforschungsabteilung } \\
\text { - } \quad \text { Geschäftsleitung, Vertriebs- } \\
\text { leitung } \\
\text { - } \quad \text { Frühere Mitarbeiter von Kon- } \\
\text { kurrenzfirmen o. Unter- } \\
\text { nehmen mit Einblick in } \\
\text { Branchenverhältnisse } \\
\text { - Einkauf } \\
\text { - F\&E } \\
\text { - Produktmanagement } \\
\text { - Personalabteilung } \\
\text { - } \quad \text { Finanz- u. Rechnungswesen } \\
\text { - Produktion } \\
\text { etc. }\end{array}$ & $\begin{array}{l}\text { - Konkurrenzanalysen } \\
\text { - } \text { Außendienstberichte } \\
\text { - Kranchenstudien } \\
\text { - Marktanalysen } \\
\text { etc. }\end{array}$ \\
\hline $\begin{array}{l}\text { Unternehmens- } \\
\text { externe Quellen }\end{array}$ & 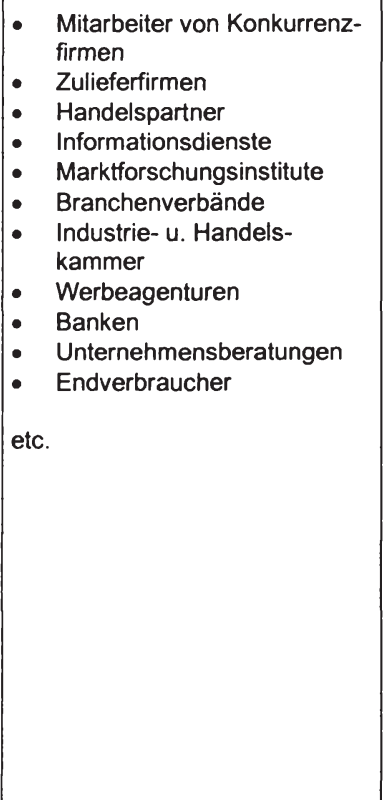 & $\begin{array}{l}\text { Tagespresse, d.h. Firmen- } \\
\text { berichte, Inserate, Stellenan- } \\
\text { zeigen } \\
\text { - } \text { Fach- u. Wirtschaftspresse } \\
\text { Konkurrenzpublikationen, } \\
\text { d.h. Hauszeitschriften, Ge- } \\
\text { schäftsberichte, Aktionärs- } \\
\text { briefe, Gebrauchsanwei- } \\
\text { sungen, Folder, Preislisten, } \\
\text { Prospekte, etc. } \\
\text { - Konkurrenzprodukte } \\
\text { Hochschulen, d.h. Vorträge, } \\
\text { Diplomarbeiten, Dissertatio- } \\
\text { nen } \\
\text { Messe- u. Ausstellungs- } \\
\text { kataloge } \\
\text { Bank- u. Börsenpublikatio- } \\
\text { nen } \\
\text { Veröffentlichungen von } \\
\text { Kammern u. Verbänden } \\
\text { Marktforschungsberichte } \\
\text { Bundesanzeiger } \\
\text { - Handelsgerichtliche Eintra- } \\
\text { gungen } \\
\text { Patente } \\
\text { etc. }\end{array}$ \\
\hline
\end{tabular}

Abbildung 19: Die Informationsquellen 248

248 Illegale Methoden werden nicht berücksichtigt. In Anlehnung an: LINK, 1988, S. 147. 


\section{I.4.3 Gewinnung von Kosteninformationen von Wettbewerbsunter- nehmen}

Nach den Ausführungen zu allgemein in der Konkurrenzanalyse verwendeten Informationsquellen im vorherigen Abschnitt, steht nun speziell die Gewinnung von Kosteninformationen von Wettbewerbsunternehmen im Mittelpunkt. Es werden Informationsquellen, die für eine Kostenbetrachtung einzubeziehen sind, ausgewählt und bewertet. Weiterführend werden die für die Kostenanalyse prädestinierten und bereits erläuterten Instrumente der Wertkettenanalyse und der Erfahrungskurve 249 in die Überlegungen einbezogen und in ein Modell integriert, das den Daten- und Informationsfluss, sowie den Einsatz von Instrumenten in der Konkurrenzanalyse beschreibt. Abschließend ist zu beurteilen, ob und wie detailliert Kosteninformationen von Konkurrenzunternehmen erfasst werden können.

Von den im vorherigen Abschnitt dargestellten Informationsquellen haben nicht alle im Zuge einer Kostenbetrachtung die gleiche Aussagekraft. Vielmehr ist der diesbezügliche Informationsgehalt der genannten Quellen sehr unterschiedlich und oft branchenabhängig. Abbildung 20 stellt eine im Allgemeinen gültige Auswahl von Quellen für die Beschaffung von Kosteninformationen dar. Die genannten Informationsquellen werden nach ihrer Bedeutung für diesen Zweck von LINK bewertet. ${ }^{250}$ Darüber hinaus nimmt der Autor eine Einschätzung vor, die teilweise von der Bedeutung abweicht, die LINK den einzelnen Informationsquellen zuweist. Differierende Bewertungen werden anschließend erläutert und begründet.

Eine herausragende Bedeutung bei der Gewinnung von Kosteninformationen von Wettbewerbern erhalten bei beiden Autoren die Quellen "Produktion" und „Konkurrenzprodukte“. Kostenrelevante Erfahrungen, die man im eigenen Unternehmen bspw. zu unterschiedlichen Produktionsverfahren gesammelt hat, können teilweise auf die Konkurrenten übertragen werden. Als Beispiel seien hier das absolute oder das stückbezogene Kostenniveau, die Kostenstruktur, aufgeteilt in fixe und variable Kosten, oder die Kostendynamik in Abhängigkeit von der Ausbringungsmenge genannt. In Verbindung mit einer genauen Ana-

249 Vgl. 3. Kapitel, Abschnitt I.3.5 und I.3.6.

250 In Anlehnung an: LINK, 1988, S. $148 \mathrm{ff}$. 
lyse der Konkurrenzprodukte, bspw. durch ein „Reverse Engineering“, bei dem das Konkurrenzprodukt vollständig zerlegt, geprüft und wieder zusammengefügt wird, können aus der Materialbeschaffenheit oder aus technischen und konstruktiven Zusammenhängen zuverlässige Rückschlüsse auf die Kosten gezogen werden.

\begin{tabular}{|l|c|c|}
\hline Informationsquelle & $\begin{array}{l}\text { Bedeutung } \\
\text { nach LiNK }\end{array}$ & $\begin{array}{l}\text { Einschätzung } \\
\text { des Autors }\end{array}$ \\
\hline Produktion & ++ & ++ \\
\hline Konkurrenzprodukte & ++ & ++ \\
\hline Geschäfts- und Vertriebsleitung & + & ++ \\
\hline Frühere Mitarbeiter von Wettbewerbern & + & 0 \\
\hline Produktmanagement & + & ++ \\
\hline Zulieferer & + & + \\
\hline Fach- und Wirtschaftspresse & + & + \\
\hline Konkurrenzpublikationen & + & + \\
\hline Bank- und Börsenpublikationen & + & + \\
\hline Bundesanzeiger & + & + \\
\hline Außendienst, Kundendienst & - & 0 \\
\hline Kunden & - & - \\
\hline Marktforschungsinstitute & - & - \\
\hline Auskunfteien & - & - \\
\hline Werbeagenturen & - & - \\
\hline Tagespresse & - & - \\
\hline++ sehr große Bedeutung \\
große Bedeutung
\end{tabular}

Abbildung 20: Die Informationquellen zur Kostenbetrachtung in der Konkurrenzanalyse

Abweichend von LINK werden die Geschäfts- und Vertriebsleitung, sowie das Produktmanagement ebenfalls zu den Informationsquellen mit einer sehr groBen Bedeutung für die Gewinnung von Konkurrenzkosteninformationen gezählt. Gewöhnlich erhält dieser Personenkreis durch seine umfangreichen Kontakte auf „beruflich-offizieller" aber auch auf persönlicher Ebene viele Brancheninformationen, die für eine Kostenbetrachtung einzelner Konkurrenten außerordentlich hilfreich sein können. Diese Informationen kommen dabei im Gespräch nicht nur von Dritten, sondern - bspw. im Rahmen eines Erfahrungsaustau- 
sches oder aus anderen Gründen - oft auch direkt von Mitarbeitern des Wettbewerbsunternehmens selbst. 251

Dabei geht es nicht um die Weitergabe direkter Kosten- oder Kalkulationsinformationen, die im Normalfall ein gut behütetes Geschäftsgeheimnis bleiben. Vielmehr sind Branchenkenner oft in der Lage, bereits auf der Basis allgemeiner, technischer oder organisatorischer Hinweise, wie bspw. Informationen über den technischen Stand bzw. die Beschaffenheit vorhandener oder geplanter Produktionsanlagen oder die ungefähre Anzahl der in einem bestimmten Bereich oder mit einem Projekt beschäftigten Mitarbeiter, Rückschlüsse auf die Effizienz und auf die Kosten fremder Produktionsabläufe im Vergleich zum eigenen Unternehmen zu ziehen. Dabei spielen die bereits genannten Erfahrungen im eigenen Produktions- und Produktbereich eine wichtige Rolle als Vergleichsmaßstab.

Früheren Mitarbeitern von Wettbewerbern wird für die Kostenanalyse in Abweichung zu LINK eine mittlere Bedeutung beigemessen. Zwar können sie oft viele Hinweise zu internen Strukturen, Prozessabläufen und Projekten des Konkurrenten geben, was entsprechende Rückschlüsse auf Kosten ermöglichen sollte, doch beziehen sich diese Informationen meist nur auf ein Unternehmen. Hinzu kommt, dass in der heutigen schnelllebigen Zeit, die vom Wandel und von permanenter Umorganisation geprägt ist, dieses Wissen schnell veraltern kann und damit seinen Informationswert verliert. Abgesehen davon beinhalten viele Arbeitsverträge von solchen Mitarbeitern, die Zugang zu sog. „sensiblen“ Informationen haben, eine Klausel, die Ihnen die Weitergabe dieser Informationen verbietet.

Der Außen- und Kundendienst wird mit einer mittleren Bedeutung für die Kostenbetrachtung von Konkurrenten bewertet, weil diese Mitarbeiter häufigen und unmittelbaren Kundenkontakt haben und daraus wertvolle Konkurrenzinformationen gewinnen können. Allerdings wird diese Möglichkeit nicht in allen Branchen gleichermaßen gegeben sein und von diversen Faktoren abhängen, wie bspw. der Frage, ob man beim Kunden Konkurrenzprodukte antrifft und im offenen Gespräch Informationen erhält, evtl. sogar von anderen Außen- und Kundendienstmitarbeitern, die ebenfalls vor Ort sind. 
Die Kostenbetrachtung von Wettbewerbsunternehmen im Rahmen der Konkurrenzanalyse sollte auf einer breiten Informationsbasis ruhen, d.h. mehrere Informationsquellen einbeziehen. Da der Informationsfluss wichtiger Quellen meist von Menschen und deren Know-how, sowie von deren beruflichen und persönlichen Kontakten abhängt, reduziert eine breite Informationsbasis die Abhängigkeit von einzelnen Personen bzw. Quellen. Darüber hinaus können gewonnene Informationen in einen Gesamtzusammenhang gestellt werden, wodurch Fehlinformationen leichter als solche zu erkennen sind. Es kommt zu einem "Mosaikeffekt", bei dem durch das Zusammenfügen vieler Einzelinformationen, die allein stehend geringere Aussagekraft besitzen, ein hinreichend genaues Bild entsteht.

Ein Instrument, mit dessen Hilfe zumindest eine Aussage über die relative Kostensituation von Wettbewerbern abgeleitet werden kann, ist die bereits vorgestellte Wertkettenanalyse. ${ }^{252}$ Je mehr allgemeine, technische oder organisatorische Informationen über Wettbewerbsunternehmen aus unterschiedlichen Quellen in diese Betrachtung einbezogen werden, desto detaillierter ist die Rekonstruktion der Wertketten von Konkurrenten möglich, wobei vorhandene Informationslücken durch „Schätzungen“253 ergänzt werden können. Auf diese Weise lässt sich für die relevanten Wettbewerber ein Gesamtbild herleiten, das die Wertaktivitäten, deren ungefähre Bedeutung für das jeweiligen Unternehmen ${ }^{254}$, sowie die Kostenantriebskräfte ${ }^{255}$ der Aktivitäten enthält.

Die Erfahrungskurve ist ein weiteres Instrument, mit dem die Kostensituation von Wettbewerbern untersucht werden kann. ${ }^{256} \mathrm{Ihr}$ Einsatz für die Kostenbetrachtung im Rahmen der Konkurrenzanalyse ist im Gegensatz zur Wertkette an diverse Voraussetzungen gebunden und unterliegt einigen Einschränkungen, die bereits an angegebener Stelle erläutert wurden und die es zu berücksichtigen gilt. Werden diese Richtlinien jedoch beachtet, ist es mit Hilfe mathematisch-statistischer Verfahren möglich, den Erfahrungskurveneffekt in Abhängigkeit von der Ausbringungsmenge abzuschätzen. Dabei können $\mathrm{Er}$ kenntnisse hilfreich sein, die auf der Basis von Daten des eigenen Unter-

\footnotetext{
252 Vgl. 3. Kapitel, Abschnitt I.3.6.

253 Welge/AL-LAHAM, 1999, S. 275; PORTER, 1999 (b), S. 103.

254 Vgl. 3. Kapitel, Abschnitt I.3.6 und vgl. ESSER, 1989, S. 203.

255 Vgl. 3. Kapitel, Abschnitt I.3.6 und vgl. PORTER, 1999 (b), S. $106 \mathrm{ff}$.

256 Vgl. 3. Kapitel, Abschnitt I.3.5.
} 
nehmens gewonnen wurden und die anschließend unter Beachtung individueller Besonderheiten auf einzelne Wettbewerber übertragen werden. Dieses vergleichende Vorgehen muss nicht gesamtunternehmensweit erfolgen, sondern kann sich auch auf bestimmte Unternehmensbereiche beschränken. Auf diese Weise hergeleitete Kosteninformationen können so mit Erkenntnissen aus der Wertkettenanalyse gespiegelt werden oder können diese teilweise ergänzen.

Obwohl gerade Kosten- und Kalkulationsinformationen meist nach außen, aber oft auch nach innen, äußerst vertraulich behandelt werden, ist generell eine zunehmend offene Informationspolitik von Untemehmen zu beobachten. ${ }^{257}$ „Bedingt durch rechtliche Verpflichtungen, aber auch hervorgerufen durch den Ruf und das Image des Unternehmens, tendieren die meisten Firmen heute zu größerer Publizitätsfreudigkeit"258. Diese Offenheit bezieht sich weniger auf die sog. „sensiblen“, sondern eher auf regelmäßig angebotene allgemeine, aber auch bereichs- und funktionsspezifische Unternehmensinformationen, die teilweise für eine kostenorientierte Analyse genutzt werden können. So kann angenommen werden, „dass der Großteil aller wünschenswerten Informationen über die Konkurrenz in der einen oder anderen Form zur Verfügung stehen“259.

Nur das Zusammenfügen vieler einzelner Wettbewerbsinformationen über einen längeren Zeitraum hinweg führt, wie dargestellt, zu einem möglichst exakten, detaillierten und verifizierten „Kostenbild“ der relevanten Konkurrenten. Da im Rahmen einer institutionalisierten Konkurrenzanalyse gewöhnlich die größte Vielfalt an Wettbewerbsinformationen zur Verfügung steht, sollte eine ausführliche Kostenanalyse von Wettbewerbern - falls sie denn vom Entscheidungsträger in einer hohen Ausführlichkeit benötigt wird - dort organisch angesiedelt sein. Hinzu kommt, dass alle Kosteninformationen im Zuge des strategischen Planungsprozesses nach der erfolgten Analysephase, zu der auch die Konkurrenzanalyse gehört, systematisch weiterverarbeitet und in unternehmerische Entscheidungen umgesetzt werden. ${ }^{260}$

257 Vgl. GÄLWEILER, 1986, S. 371 und S. 384.

258 KREIKEBAUM, 1997, S. 121.

259 KREIKEBAUM, 1997, S. 121.

260 Zum Prozess der strategischen Planung vgl. 1. Kapitel. 
Auf der Grundlage diverser Informationsquellen und mit Hilfe verschiedener Instrumente kann eine gewisse Transparenz der Kostensituation erreicht werden, die Branchenkenner in der Lage versetzen sollte, durch eine vergleichende Analyse mehrerer Wettbewerber relative Kostenpositionen herauszufinden. ${ }^{261}$ Darüber hinaus ist es durchaus realistisch, dass detailliertere Kosteninformationen, wie bspw. Kostenvor- oder -nachteile in bestimmten Unternehmens- oder Produktionsbereichen abgeleitet werden können. Diese werden sich teilweise sogar recht genau quantifizieren lassen, so dass abschließend davon ausgegangen werden kann, dass - abhängig vom Einzelfall - Kosteninformationen von Wettbewerbsunternehmen beschafft werden können.

Zusammenfassend wird der Daten- und Informationsfluss, sowie der Einsatz von Instrumenten in der Konkurrenzanalyse in Abbildung 21 dargestellt:

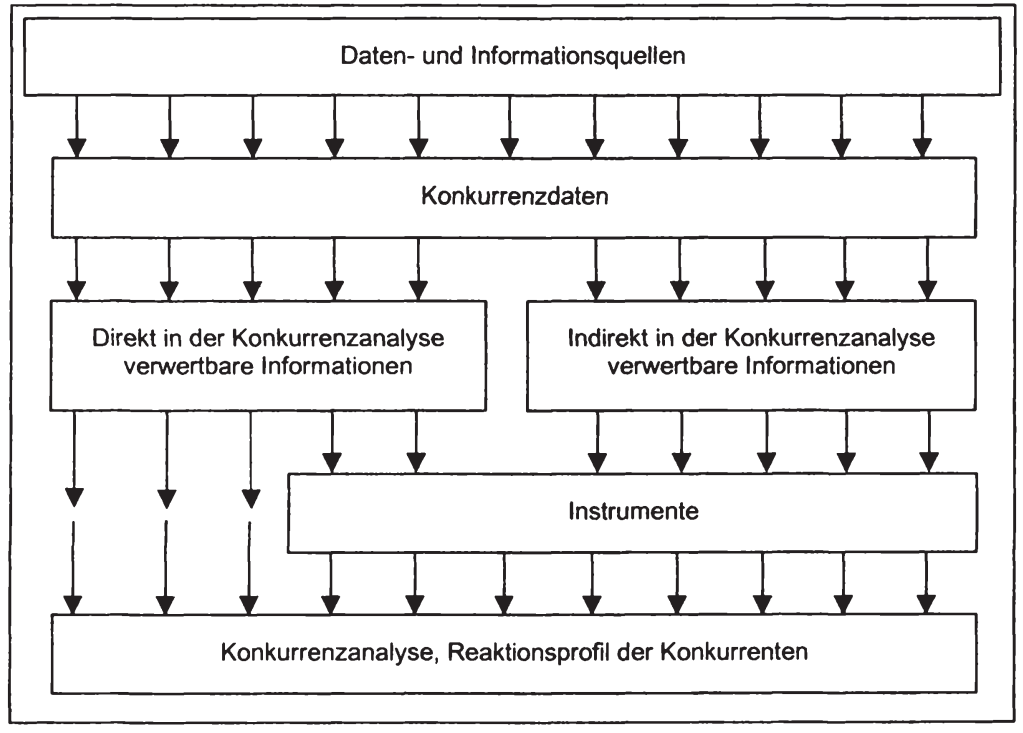

Abbildung 21: Der Daten- und Informationsfluss in der Konkurrenzanalyse

Aus einer Vielzahl von Quellen werden Daten erhoben, die direkt oder indirekt in der Konkurrenzanalyse verwertbar sind. Wegen ihrer Zweckorientierung können diese Daten als Informationen bezeichnet werden. Ein Großteil der Kon- 
kurrenzinformationen wird mit Hilfe von Instrumenten erfasst, zusammengeführt oder aufbereitet. Nur ein Teil der Informationen kann direkt über die Konkurrenzanalyse verarbeitet und in strategische Überlegungen einbezogen werden, wie bspw. die Marktpreise für benötigte Produktionsfaktoren. Ebenso sind Absatz- und Marktanteilsinformationen von Wettbewerbern direkt verwertbar, da sie einen Hinweis auf die Größe oder auf die Bedeutung eines Unternehmens liefern. Werden diese Informationen darüber hinaus auf ihre Auswirkungen hinsichtlich des Erfahrungskurveneffektes untersucht, können ferner, wie bereits erläutert, Kosteninformationen abgeleitet werden. Ähnliches gilt für viele technische und organisatorische Informationen, über die mit Hilfe der Wertkettenanalyse ebenfalls eine Kostenanalyse von Wettbewerbsunternehmen durchgeführt werden kann. Alle Konkurrenzinformationen zusammen ergeben ein Gesamtbild der relevanten Wettbewerber, das je nach individuellem Informationsbedarf von Entscheidungsträgern bis hin zu einem Reaktionsprofil, in Anlehnung an PORTER, verfeinert werden kann. 


\section{Kapitel Kostenbetrachtungen in der Konkurrenzanalyse}

Der Betrachtung von Kosten im Rahmen der Konkurrenzanalyse wird im Schrifttum eine unterschiedliche Bedeutung und damit eine variierende strategische Relevanz beigemessen, wie im 1. Abschnitt dieses Kapitels dargelegt wird. Ein Ziel der vorliegenden Arbeit ist es, einen Beitrag zur Auflösung dieser Kontroverse zu leisten. Zu diesem Zweck wird im 2. Abschnitt eine Grundhypothese formuliert, die die Basis für die im Teil II dieser Arbeit durchzuführende Untersuchung bildet. Zuvor werden nachfolgend einige begriffliche Erläuterungen vorgenommen.

Kosten lassen sich allgemein definieren als bewerteter Güter- und Leistungsverzehr, der für die betriebliche Leistungserstellung eines Unternehmens benötigt wird. ${ }^{262}$ Kosten sind die grundlegende Rechengröße der Kosten- und Leistungsrechnung, die dem internen Rechnungswesen zugeordnet ist ${ }^{263}$ und die vorwiegend - allerdings nicht nur - Informationen für die unternehmensinterne Verwendung liefert, wie bspw. für die Wirtschaftlichkeits- und Erfolgskontrolle oder zur Unterstützung unternehmerischer Entscheidungen. ${ }^{264}$ Das Rechnungswesen, welches sich u.a. in ein internes und in ein externes Rechnungswesen gliedern lässt, wird als ein System zur quantitativen Ermittlung, Aufbereitung und Darstellung von wirtschaftlichen Zuständen zu einem bestimmten Zeitpunkt und von wirtschaftlichen Abläufen während eines Zeitraumes verstanden. 265

Kosten sind unmittelbar ergebniswirksam, denn durch Saldierung aller Kosten und Leistungen eines Zeitraumes ergibt sich das Periodenergebnis. Kosten haben folglich einen direkten Einfluss auf die Erreichung von Erfolgs- oder Renditezielen eines Unternehmens und damit auch auf dessen gewöhnlich übergeordnetes Ziel, der langfristigen Sicherung der Unternehmensexistenz.

262 Vgl. COENENBERG, 1999, S. 39. Eine kurze Übersicht über unterschiedliche betriebswirtschaftliche Kostenbegriffe, sowie die Abgrenzung gegenüber anderen Grundbegriffen des Rechnungswesens finden sich bei KÜPPER, 1998, S. $458 \mathrm{ff}$.

263 Vgl. Kussmaul, 2000, S. 59 ff.; TROBMANn, 1999, S. $307 \mathrm{ff}$.

$264 \mathrm{Zu}$ den Aufgaben der Kosten- und Leistungsrechnung vgl. COENENBERG, 1999, S. 37 f.; KUSSMAUL, 2000, S. $59 \mathrm{f}$.

265 Vgl. BUSSE VON COLBE, 1998, S. $599 \mathrm{ff}$. 
Weiterhin beeinflussen Kosten das Verhalten von Unternehmen innerhalb ihres Wettbewerbsumfeldes. Niedrige Kosten bilden neben der Differenzierung die beiden „Grundtypen von Wettbewerbsvorteilen“266. Deren Nutzung erfolgt durch die bereits erläuterten generellen Wettbewerbsstrategien Kostenführerschaft, Differenzierung und Konzentration auf Schwerpunkte. ${ }^{267}$ Durch die Höhe von Kosten, aber bspw. auch durch deren Anpassungsfähigkeit an unterschiedliche Beschäftigungsgrade, die in der Struktur, aufgeteilt in fixe und variable Kostenanteile, zum Ausdruck kommen kann, ergeben sich Wettbewerbsvor- oder -nachteile auf der Kostenebene. Die bewusste Beeinflussung von Kosten durch produkt-, prozess- oder ressourcenorientierte Maßnahmen im Kostenmanagement ist damit von besonderer Wichtigkeit. ${ }^{268}$ Werden Wettbewerbsvorteile auf der Kostenebene genutzt oder Nachteile durch eine entsprechende strategische Positionierung gemindert, äußert sich dies in strategischen Entscheidungen.

Kosten erlangen somit eine strategische Bedeutung: Sie tragen unmittelbar zur Erreichung wesentlicher Unternehmensziele bei, sie bilden teilweise die Grundlage von Wettbewerbsvor- oder -nachteilen und beeinflussen folglich die eigene, aber entsprechend auch die Strategie von Wettbewerbern. Während die wichtige Bedeutung der Kostenanalyse im Rahmen der meist unternehmensintern orientierten Kostenrechnung seit langem ein fester Bestandteil der allgemeinen betriebswirtschaftlichen Erkenntnisse ist, gibt es eine unterschiedliche Bewertung im Schrifttum über die Bedeutung einer Kostenanalyse von Wettbewerbsunternehmen. Diese Kontroverse wird im folgenden Abschnitt dargestellt.

\subsubsection{Unterschiedliche Bedeutung von Kosteninformationen in der Konkurrenzanalyse im Schrifttum}

Kosten sind, wie zuvor erläutert, von strategischer Bedeutung. Über die eigenen Kosten informiert die Kostenrechnung, während die Kostenanalyse von Wettbewerbsunternehmen im Rahmen einer institutionalisierten und systematisch angelegten Konkurrenzanalyse erfolgen kann. ${ }^{269}$

266 PORTER, 1999 (b), S. 37.

267 Vgl. 1. Kapitel, Abschnitt I.1.3.3.

268 Vgl. FRANZ/KAJÜter, 1997, S. 8; Franz/KAJÜter, 2000 (a), S. $103 \mathrm{ff}$.

269 Vgl. 4. Kapitel, Abschnitt I.4.2. 
Die Berücksichtigung von Kosteninformationen von Wettbewerbern im Rahmen der Konkurrenzanalyse findet in der Literatur jedoch unterschiedliche Beachtung. In einem Spektrum, das den Intensitätsgrad der Kostenbetrachtung in der Konkurrenzanalyse zu Grunde legt, lassen sich drei Kategorien feststellen:

1. Zur Betrachtung von Kosten im Rahmen der Konkurrenzanalyse werden vom jeweiligen Autor keinerlei Aussagen gemacht. Kosteninformationen über Wettbewerber fließen damit nicht in die Konkurrenzanalyse und in den Prozess der strategischen Planung ein.

2. Kosteninformationen von Konkurrenzunternehmen werden als ein Bestandteil der Konkurrenzanalyse angesehen.

3. Kosteninformationen von Wettbewerbern sind ein wesentlicher Bestandteil der Konkurrenzanalyse und beeinflussen die Strategieformulierung im Rahmen der strategischen Unternehmensplanung.

Die Abgrenzung zwischen der ersten und der zweiten Kategorie lässt sich eindeutig bestimmen. Die zweite und dritte Kategorie hingegen sind durch einen fließenden Übergang charakterisiert, der sich durch die Intensität, mit der Kosteninformationen von Wettbewerbern über die Konkurrenzanalyse in den Strategieprozess einbezogen werden, auszeichnet.

ad 1.) Keine Aussage zu Kosteninformationen im Rahmen der Konkurrenzanalyse

Wettbewerbsunternehmen betrachtet ULRICH ausgehend von der Stärken-I Schwächen-Analyse des eigenen Unternehmens. ${ }^{270}$ Als Beurteilungsmaßstab wählt ULRICH u.a. den Konkurrenzvergleich und untersucht einzelne Merkmale aus den Bereichen Ergebnisse, Leistungspotential, Strategien und Unternehmungsführung, die er in einem Stärken-/Schwächen-Profil abbildet ${ }^{271}$. ULRICH macht in diesem Zusammenhang keine Aussage zu Kostenbetrachtungen im Rahmen der Wettbewerbsanalyse und wird daher der ersten Kategorie zugeordnet.

270 Vgl. ULRICH, 1990, S. $62 \mathrm{ff}$.

271 Das Stärken-/Schwächen-Profil in der Konkurrenzanalyse wird im 3. Kapitel, Abschnitt I.3.1, ausführlich dargestellt. 
Zu den Autoren, die Kosteninformationen in ihrer Darstellung der Konkurrenzanalyse nicht erwähnen, gehört HOFFMANN, dessen Checkliste zur Konkurrenzanalyse im 3. Kapitel, Abschnitt 1.3.2, dargestellt ist. ${ }^{272}$ Sie zeigt „wichtige Elemente einer Stärken-/Schwächen-Analyse der Konkurrenz"273, wobei je nach Aufgabenstellung einzelne Elemente wegzulassen seien, um den Arbeitsumfang in Grenzen zu halten. Da HofFMANN an keiner Stelle seiner ausführlichen Darstellung Kosteninformationen von Konkurrenzunternehmen erwähnt, fallen seine Ausführungen in die erste Kategorie.

Die Beobachtung und Analyse von Konkurrenzunternehmen stehen im Mittelpunkt des Fragenkataloges von GRoss, der "Ansatzpunkte für eine gezielte Abhebung von der Konkurrenz und die Erreichung einer starken Marktstellung"274 aufzeigen will. GRoss stellt in seiner Checkliste hintergründige Fragen zu vielen Bereichen des Wettbewerbs, wobei die Kosten von Konkurrenzunternehmungen keine Berücksichtigung finden. Er wird damit ebenfalls der ersten Autorengruppe zugeordnet.

ad 2.) Kosteninformationen von Konkurrenzunternehmen sind ein Bestandteil der Konkurrenzanalyse

HAMMER, dessen konkurrenzanalytischer Ansatz im 3. Kapitel, Abschnitt 1.3.1, detailliert vorgestellt wird, betrachtet die „Kostensituation“275 des eigenen Unternehmens im Vergleich zu Konkurrenzunternehmen. Zur Analyse der Kostensituation untersucht HAMMER die Produktions-, Vertriebs- und Verwaltungsgemeinkosten, die Beschaffungskosten, Kosten für die Lagerhaltung, Personalkosten und Rationalisierungsmöglichkeiten. ${ }^{276}$ In seinen weiteren Ausführungen geht HAMMER nicht auf strategiebeeinflussende Folgerungen aus der Kenntnis der Kostensituation ein, weshalb er der zweiten Autorengruppe zugerechnet wird.

\footnotetext{
272 Vgl. HOFFMANN, 1986, S. $202 \mathrm{f}$.

273 HOFFMANN, 1986, S. 201.

274 GROSS, 1974, S. 1.

275 HAMMER, 1995, S. 40.

276 Vgl. HAMMER, 1995, S. 41.
} 
GÄLWEILER schließt in seinem „Arbeitsblatt zur Konkurrenzanalyse“ ebenfalls die „Kostensituation“277 ein. Zusätzlich betrachtet er darin „Kostenvorteile“, die jedoch nicht genauer erläutert werden. Kosteninformationen von Konkurrenzunternehmen zählen bei GÄLWEILER nicht zu den wesentlichen Inhalten einer Konkurrenzanalyse. ${ }^{278} \mathrm{Im}$ weiteren Verlauf seiner Ausführungen geht GÄLWEILER auf "Ergebnis- und Kosten-Analysen“ ein, deren Schwerpunkt „die bessere Kenntnis der im operativen Geschäft ständig ergebniswirksamen technischökonomischen Einflussgrößen aller Art"279 seien. GÄLWEILER nennt hierbei u.a. die „Kostenstruktur-Analyse im Vergleich mit Konkurrenzunternehmen“280, wobei er nicht weiter auf diesen Begriff eingeht. Zusammenfassend kann gesagt werden, dass GÄLWEILER Kosteninformationen von Wettbewerbern in der Konkurrenzanalyse berücksichtigt, sie jedoch nicht eindeutig als strategierelevant bezeichnet. Seine Beiträge werden daher der zweiten Kategorie zugeordnet.

Berücksichtigung finden Kosteninformationen von Wettbewerbsunternehmen auch in der von HINTERHUBER dargestellten Konkurrenzanalyse. ${ }^{281}$ In einem "Gliederungsschema der Umweltanalyse und -prognose“282 betrachtet HINTERHUBER die „Kostensituation" der Unternehmung. Dazu analysiert er die Standorte mit Bezug auf die Energie- und Rohstoffversorgung, die Arbeitskräfte, die Absatzmärkte, etc.. Darüber hinaus wird die relative Effizienz des Produktionsund Distributionsapparates im Sinne der Erfahrungsökonomie mit Blick auf die Kosten der Wettbewerber untersucht. Da HINTERHUBER nicht eindeutig auf eine strategierelevante Bedeutung von Kosteninformationen von Wettbewerbsunternehmen eingeht, wird er ebenfalls der zweiten Kategorie zugeordnet.

ad 3.) Kosteninformationen über Wettbewerbsunternehmen als ein wesentlicher Bestandteil der Konkurrenzanalyse mit strategischer Relevanz

Zu den Autoren, die dieser Kategorie zuzuordnen sind, zählt PORTER, dessen System zur Konkurrenzanalyse ausführlich im 3. Kapitel, Abschnitt 1.3.4, dargestellt wird. Darin werden u.a. die Fähigkeiten der Wettbewerber untersucht,

277 GÄLWEILER, 1986, S. 372.

278 Vgl. GÄLWEILER, 1986, S. 370 f. und vgl. 3. Kapitel, Abschnitt I.3.2.

279 GÄLWEILER, 1986, S. 385.

280 GÄLWEILER, 1986, S. 385.

$281 \mathrm{Vgl}$. HINTERHUBER, 1996 (a), S. $116 \mathrm{ff}$.

282 HINTERHUBER, 1996 (a), S. $118 \mathrm{f}$. 
strategische Schritte zu ergreifen, wofür deren Stärken und Schwächen ausschlaggebend sind.283 PORTER geht bei der Bestimmung von Stärken und Schwächen ausführlich in Form von Checklisten und mit Hilfe vertiefender Fraugen auf die Kosten von Konkurrenzunternehmen ein. ${ }^{284}$ Das Grundinstrument zur Ermittlung von Kosteninformationen von Konkurrenten ist die WertkettenAnalyse, mit der PORTER die relative Kostenposition eines Konkurrenten ermittelt. 285 Führt ein Unternehmen strategisch relevante Aktivitäten kostengünstiger aus als andere Anbieter, entstehen Wettbewerbsvorteile, die strategisch nutzbar sind.286 PORTER geht ausführlich auf die strategischen Möglichkeiten ein, die sich durch eine günstige relative Kostenposition eröffnen ${ }^{287}$ und wird damit eindeutig der dritten Kategorie zugerechnet.

Auch LINK beschäftigt sich mit der strategischen Bedeutung von Kosteninformationen in der Konkurrenzanalyse.288 Neben der Berücksichtigung von „Kostenstrukturen“ im bereits unter Abschnitt I.3.2 im 3. Kapitel erwähnten „Variablenkatalog zur Charakterisierung von Konkurrenzunternehmen“289 stellt LINK einen an gleicher Stelle vorgestellten Fragenkatalog auf, der zur Ermittlung von Kostenvorteilen dient. Diese beeinflussen die Wettbewerbsfähigkeit, die jedoch auch von anderen Faktoren, wie z.B. von der Innovationsbereitschaft, der Kompetenz oder der Mitarbeitermotivation bestimmt wird. Unter der Voraussetzung, dass auch diese Faktoren in die Konkurrenzanalyse einfließen, kann die Betrachtung von Kostenvorteilen „Zu interessanten Aufschlüssen“290 führen. Bezugnehmend auf PORTER verweist LINK in seinen Ausführungen auf die Strategie der Kostenführerschaft.

Unter dem Oberbegriff „Strategic Management Accounting“ beschreibt COENENBERG eine strategieorientierte Kostenrechnung, die interne und externe Informationen erfasst und verarbeitet, welche für die Lösung von strategischen Pro-

\footnotetext{
283 Vgl. PORTER, 1999 (a), S. $105 \mathrm{ff.}$

284 Die Kostenanalyse wird detailliert im 3. Kapitel, Abschnitt 1.3.4, behandelt.

285 Vgl. PORTER, 1999 (b), S. 142 f. Die Wertkettenanalyse wird außerdem im 3. Kapitel, Abschnitt I.3.6, dargestellt.

286 Vgl. PORTER, 1999 (b), S. 63.

287 Vgl. PORTER, 1999 (b), S. 38 ff. und S. 97 ff.

288 Vgl. LiNK, 1988, S. $123 \mathrm{ff}$.

289 LINK, 1988, S. 87.

290 LINK, 1988, S. 128.
} 
blemsituationen relevant sind.291 Von einer strategieorientierten Kostenrechnung sind u.a. relative Kosten und Preise, sowie Absatzmengen, Marktanteile, etc. zu ermitteln und im Vergleich zu Konkurrenzunternehmen zu präsentieren. Durch den Einbezug wettbewerbsorientierter Informationen wird „eine systematische Untersuchung der eigenen Wertschöpfungsaktivitäten im Hinblick auf mögliche Wettbewerbsvorteile"292 ermöglicht. Die Aussagen COENENBERGS berücksichtigen Kosteninformationen von Konkurrenzunternehmen und beziehen diese in die Überlegungen zur Auswahl strategischer Handlungsalternativen ein. 293

Zusammenfassend wird deutlich, dass die Berücksichtigung von Kosteninformationen von Wettbewerbern im Rahmen der Konkurrenzanalyse in der Literatur nicht einheitlich erfolgt. Während einige Autoren in ihren Ausführungen zur Konkurrenzanalyse Kosteninformationen über Wettbewerber gar nicht erfassen, erlangen diese Informationen bei anderen Autoren eine strategierelevante Bedeutung.

\section{I.5.2 Untersuchung der Bedeutung von Kosteninformationen in der Konkurrenzanalyse}

Nachdem im vorherigen Abschnitt die unterschiedliche Bedeutung von Kosteninformationen von Wettbewerbern in der Konkurrenzanalyse dargestellt worden ist, wird im noch folgenden Teil II der vorliegenden Arbeit eine entsprechende Untersuchung zu dieser Problematik durchgeführt. Dazu wird in diesem Abschnitt eine Grundhypothese erarbeitet, die einerseits die Fragestellung konkretisiert und die andererseits den Ausgangspunkt für die anschließende Untersuchung bildet.

291 Vgl. COENENBERG, 1999, S. 42, in Anlehnung an: BROMWICH, 1990, S. 27 ff.; Simmonds, 1989, S. $264 \mathrm{ff}$.

292 COENENBERG, 1999, S. 42.

293 Basierend auf den Ausführungen von PORTER, 1999 (b), S. 166 f. und STEINMANN/GUTHUNZ/HASELBERG, 1992, S. 1459 schlagen WELGE/AMSHOFF eine Vorgehensweise für eine

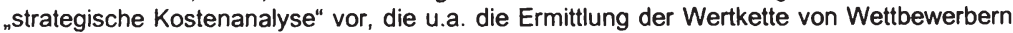
mit dem Ziel vorsieht, relative Kostenpositionen und die Quelle von Kostenunterschieden festzustellen, um darauf aufbauend strategische Entscheidungen zu fällen. Wegen der Ähnlichkeit zu den Ausführungen COENENBERGS, wird an dieser Stelle nur auf den Ansatz von WELGE/AMSHOFF verwiesen. Vgl. WELGE/AMSHOFF, 1997, S. $59 \mathrm{ff}$. 
Auf der Basis der bisherigen, im Teil I eher theoretisch geprägten Ausführungen, lässt sich besonders ein Vorteil der Kostenbetrachtung in der Konkurrenzanalyse herausstellen: Die genaue Kenntnis eigener und fremder Wettbewerbsvorteile und -nachteile auf der Kostenebene. Erst dieses Wissen ermöglicht es, Stärken und Schwächen, die u.a. relativ zu den Wettbewerbern zu sehen sind, festzustellen.294 So können eigene Stärken auf der Kostenseite bewusst eingesetzt und genutzt werden. Andererseits erlaubt es die Kenntnis entsprechender fremder Stärken und Schwächen, sein eigenes Verhalten im Wettbewerb darauf einzustellen. Darüber hinaus kann dieses Wissen dazu beitragen, Entscheidungen von Wettbewerbern zu antizipieren. ${ }^{295}$ Szenarien, die Aktionen und Reaktionen von Wettbewerbern prognostizieren, können so genauer auf ihre Eintrittswahrscheinlichkeit hin überprüft werden. Strategische Entscheidungen, die auf einer sicheren Erkenntnis - nicht auf einer wagen Vermutung - über die eigenen Stärken und Schwächen auf Kostenebene beruhen, sind demnach unter geringerer Unsicherheit zu fällen.

Klassifiziert man Unternehmen nach ihrer verfolgten generellen Wettbewerbsstrategie Kostenführerschaft, Differenzierung und Konzentration auf Schwerpunkte $^{296}$ und überträgt darauf die oben angestellten Überlegungen, ist die Bedeutung der Kosteninformationen über Wettbewerber für Kostenführer größer, als für Unternehmen, die eine der beiden anderen Wettbewerbsstrategien verfolgen. Weder bei der Einzigartigkeit eines Produktes bzw. einer Leistung im Falle der Differenzierung, noch bei der Besetzung einer Marktnische, die bei der Konzentration auf Schwerpunkte erfolgt, sind die Entscheidungsträger derart auf die Kenntnis von Stärken und Schwächen auf der Kostenebene angewiesen, wie bei der Kostenführerschaft, bei der die Minimierung der Kosten im Vordergrund steht.

Die Kenntnis - nicht die Vermutung - von Stärken oder Schwächen auf der Kostenebene haben für Kostenführer eine größere Wichtigkeit, weil ihre gesamte strategische Ausrichtung darauf basiert, einen Kostenvorsprung im Vergleich zu den Wettbewerbern zu erlangen. Diese Kenntnis brancheninterner

294 Zur Ermittlung von Stärken und Schwächen siehe 1. Kapitel, Abschnitt 1.1.3.2.

295 Diesbezüglich sei auf die Ausführungen zum System der Konkurrenzanalyse nach PORTER im 3. Kapitel, Abschnitt 1.3.4, verwiesen.

296 Basierend auf PORTER, 1999 (a), S. 75 werden im 1. Kapitel, Abschnitt 1.1.3.3, die generellen Wettbewerbsstrategien im Rahmen der Strategieformulierung dargestellt. 
Kostenvor- und -nachteile bedeutet in diesem Fall insofern eine Zunahme an Planungs- und Entscheidungssicherheit.

Die Grundsatzentscheidung für eine Kostenführerstrategie ist mit diversen Folgeentscheidungen verbunden. ${ }^{297}$ Der Zwang zur Kostenminimierung führt im Bereich der variablen Kosten, dem Erfahrungskurvenkonzept folgend, zu einer Massenstrategie. Mit dieser gehen zusätzlich eine Fixkostendegression je Stück, sowie evtl. Einsparungsmöglichkeiten auf den Beschaffungsmärkten wegen der gestiegenen Einkaufsmacht einher, so dass auf mehreren Seiten Kosteneinsparungen erreicht werden können. Wird die Massenstrategie vom Kostenführer eingesetzt, was nicht zwingend der Fall sein muss, wofür jedoch viele Argumente sprechen, ist diese mit einer Betriebsvergrößerung bzw. mit einem Aufbau von Produktionskapazitäten verbunden. Die in Folge dessen notwendige Steigerung des Marktanteils kann durch ein aggressives Marketing, insbesondere mit Preissenkungen erreicht werden, die von einem Kostenführer aufgrund seiner günstigen Kostenposition effektiver eingesetzt werden können, als von Wettbewerbern, die zu höheren Kosten produzieren. Gerade in dieser Situation ist es nützlich zu wissen, welcher Wettbewerber aufgrund seiner Kostensituation niedrige Preise dauerhaft mithalten kann. In den Bereichen F\&E, Service, Vertrieb und Werbung ist eine Kostenführerstrategie gewöhnlich mit einer strikten Kostenkontrolle verbunden, wobei durchaus auch auf diesem Weg eine aggressive Marketingpolitik zur Steigerung des Marktanteils gewählt werden kann.

Wenn all diese Folgeentscheidungen, die mit einer Kostenführerstrategie einher gehen, von der Unternehmensführung zu fällen sind und die Kenntnis der Stärken und Schwächen auf der Kostenebene zu einer Zunahme an Planungsund Entscheidungssicherheit führt, dann stellt sich die Frage, ob dies bestimmte strategische Entscheidungen und damit letztlich den Unternehmenserfolg beeinflusst. Aus dieser Überlegung abgeleitet soll folgende Grundhypothese formuliert werden: Die Kenntnis der Stärken und Schwächen auf der Kostenebene, welche durch eine Kostenbetrachtung in der Konkurrenzanalyse abgeleitet werden können, führt insbesondere bei Kostenführern zu einer Zunahme an

297 Siehe dazu die grundlegenden Ausführungen im 1. Kapitel, Abschnitt 1.1.3.3.

Christoph Heinen - 978-3-631-75315-6 
Planungs- und Entscheidungssicherheit und beeinflusst damit strategische Entscheidungen und den wirtschaftlichen Unternehmenserfolg.

Im anschließenden Teil II der vorliegenden Arbeit soll diese Grundhypothese mit Hilfe einer empirischen Untersuchung überprüft werden. Falls sich die geschilderten Zusammenhänge bestätigen, führt dies im Teil III zu der Forderung, die Kostenbetrachtung als festen Bestandteil der Konkurrenzanalyse anzusehen. 
Christoph Heinen - 978-3-631-75315-6

Downloaded from PubFactory at 01/11/2019 06:18:34AM

via free access 


\section{Teil II Eine empirische Untersuchung zur Bedeutung von Kosteninformationen in der Konkurrenz- analyse}

Im Rahmen dieser Arbeit wird untersucht, ob die Kenntnis von Stärken und Schwächen auf der Kostenebene, die durch eine Kostenbetrachtung in der Konkurrenzanalyse abgeleitet werden können, strategische Entscheidungen und damit den wirtschaftlichen Unternehmenserfolg beeinflusst.

Nach den grundlegenden, eher theoretisch geprägten Ausführungen im Teil I steht im Teil II eine empirische Studie im Mittelpunkt, mit deren Hilfe die Bedeutung von Kosteninformationen in der Konkurrenzanalyse betrachtet und bewertet werden soll. Es ist das Ziel der Studie, Aussagen zu diesen Fragestellungen zu entwickeln, wobei ein möglichst enger Bezug zur Realität, d.h. zur Unternehmenspraxis geschaffen werden soll. Dies wird durch den Einsatz eines Unternehmensplanspiels erreicht.

Die Vorgehensweise im Teil II lässt sich folgendermaßen skizzieren: In einem ersten Schritt, im 6. Kapitel, wird der experimentelle Einsatz von Unternehmensplanspielen im Rahmen wissenschaftlicher Studien erörtert. Dabei wird die Verwendung eines Planspiels auch für die vorliegende Untersuchung empfohlen. Das 7. Kapitel beschäftigt sich mit der Auswahl eines geeigneten Simulationsmodells. Zu diesem Zweck wird zuerst ein Katalog relevanter Selektionskriterien aufgestellt. Anschließend wird die Auswahl des General Management Planspiels MARGA begründet und das Simulationsmodell in diesem Zusammenhang gleichzeitig vorgestellt und erläutert. Nach der Herleitung der inhaltlichen Problematik im Teil I und der anschließenden Auswahl des entsprechenden Untersuchungsinstruments, des Planspiels MARGA, bildet der im 8. Kapitel dargestellte Versuchsaufbau die Verbindung zwischen Inhalt und Instrument. Dabei werden das Instrumentarium und die Versuchsanordnung an die inhaltlichen Erfordernisse der Untersuchung angepasst. Anschließend erfolgt die Formulierung der Hypothesen, die im weiteren Verlauf der Ausführungen den Ansatzpunkt für die mathematisch-statistischen Verfahren darstellen. Die zum Einsatz kommenden Verfahren werden im 9. Kapitel 
vorgestellt. Gegenstand des 10. Kapitels sind die Anwendung der statistischen Verfahren auf das erfasste Datenmaterial und die hypothesenweise Darstellung der Untersuchungsergebnisse.

Im darauf folgenden Teil III stehen die Interpretation und die Bewertung der Untersuchungsergebnisse und die daraus abzuleitenden Forderungen für die Konkurrenzanalyse im Mittelpunkt. 


\section{Kapitel Experimentelle Wirtschaftsforschung anhand von Unternehmensplanspielen}

Zur Untersuchung der bereits erläuterten Zusammenhänge reichen theoretische Überlegungen, die im Teil I angestellt werden, nicht aus. Sie können - und müssen - jedoch die Basis für eine realitätsbezogene Analyse der Fragestellungen sein. Zur Herstellung des geforderten Realitätsbezuges sind tatsächliche Entscheidungen von Wirtschaftssubjekten einzubeziehen. Dafür stehen mehrere Instrumente zur Auswahl:

- Die Untersuchung realer Unternehmen bspw. mit Hilfe persönlich geführter Interviews, Befragungen mittels postalisch versendeter Fragebögen oder die Analyse öffentlich zugänglicher Informationen.

- Die Entwicklung einer Fallstudie, die einer Anzahl von Probanden eine konstruierte Situation vorgibt. Die dabei zu beobachtenden Entscheidungen werden anschließend ausgewertet. Dieses Vorgehen ist mit einem vereinfachten Laborexperiment vergleichbar, bei dem Entscheidungen von Probanden, die sich in einer spezifischen Entscheidungssituation befinden, beobachtet werden.

- Die Verwendung eines Unternehmensplanspiels. Dabei befinden sich Teilnehmer - ausgehend von einer vorgegebenen Situation - in der Rolle der Unternehmensleitung und haben diverse unternehmerische Entscheidungen innerhalb eines simulierten Geschäftszeitraumes für ihr Unternehmen zu fällen. Die Entscheidungen und deren Auswirkungen werden erfasst und hinterher ausgewertet.

Die Untersuchung der aufgeworfenen Fragestellungen am Beispiel realer Unternehmen lässt insbesondere für die vorliegende Studie einige Schwierigkeiten erwarten:

- Jedes betrachtete reale Unternehmen befindet sich in einer individuellen Situation mit einer eigenen Geschichte, eigenen Stärken und Schwächen, die das Entscheidungsverhalten des Managements beeinflussen. Die Beobachtung des Verhaltens ermöglicht damit nur eine Analyse von Einzelfällen, eine empirische Vergleichbarkeit der Einzelfälle lässt sich jedoch nicht zwingend ableiten. 
- Das für die vorliegende Studie zu beschaffende Datenmaterial ist in der Regel nicht offen zugänglich und wird von den Unternehmen teilweise als "sensibel“, d.h. als „nicht für Außenstehende bestimmt" eingestuft. Insofern wäre die Erhebung der Daten abhängig von der Bereitschaft zur Offenlegung interner strategischer Entscheidungsprozesse.

- Ein auch nur geringer Unterschied in der Bereitschaft zur Offenheit einzelner Unternehmen, der zu erwarten wäre, würde zu qualitativen Unterschieden im Datenmaterial führen, wodurch die Vergleichbarkeit nicht mehr gegeben sein würde.

- Es kann nicht ausgeschlossen, sondern muss vielmehr als wahrscheinlich angesehen werden, dass eine Analyse in unterschiedlichen Branchen zu jeweils anderen Untersuchungsergebnissen führen würde. Eine Vergleichbarkeit der Daten und eine Allgemeingültigkeit der daraus resultierenden Ergebnisse sind auf diese Weise nicht zu erreichen.

Die genannten Schwierigkeiten, die letztlich daraus resultieren, dass die durchzuführende Studie auf Daten basiert, die von den Unternehmen als "sensibel“ eingestuft werden, würden zu einer Betrachtung von Einzelfällen führen. Die allgemeine Aussagekraft der Studie wäre dadurch stark eingeschränkt, so dass eine Datenerhebung auf der Basis real existierender Unternehmen nicht in Enwägung gezogen wird.

Die Entwicklung und Anwendung einer Fallstudie, die zur Durchführung eines vereinfachten Laborexperimentes führen würde, stellt eine weitere Möglichkeit dar, die Problemstellung dieser Arbeit auf der Grundlage realer Entscheidungen von Wirtschaftssubjekten zu untersuchen und damit den gewünschten Realitätsbezug zu erreichen.

In Bezug auf die Problemstellung ist bei der Anwendung dieses Instrumentes ein besonders kritischer Aspekte zu beachten: Die Problemstellung setzt bei der Berücksichtigung von Kosten in der Konkurrenzanalyse an. Daraus wird die Kenntnis von Wettbewerbsstärken und -schwächen auf der Kostenebene abgeleitet. Diese Kenntnis beeinflusst, so die Grundannahme, strategische Entscheidungen und den wirtschaftlichen Unternehmenserfolg. Die Komplexität dieser Zusammenhänge, die auf einer dynamischen Wettbewerbssituation eines längeren Zeitraum aufbaut, lässt sich in einer Fallstudie nur unzureichend abbilden. Aus diesem Grund wird auch diese Möglichkeit verworfen 
Ein weiteres Instrument zur Schaffung des notwendigen Realitätsbezuges ist die experimentelle Verwendung eines Unternehmensplanspiels.

Unternehmensplanspiele 298 sind „modellhafte Abbildungen von Unternehmen oder Teilbereichen davon“. Sie „simulieren abstrahiert, aber dennoch realitätsnah, Gesamtabläufe und Zielkonflikte in einem Unternehmen"299.

Unternehmensplanspiele werden seit $1957^{300}$ in der Aus- und Weiterbildung, aber auch in der experimentellen Wirtschaftsforschung eingesetzt. ${ }^{301}$ Zur experimentellen Wirtschaftsforschung werden im Rahmen der vorliegenden Studie - SAUERMANN/SELTEN folgend 302 - solche Experimente gerechnet, die das ökonomische Verhalten von Versuchspersonen beobachten.

Die Modellsituation auch eines komplexen Unternehmensplanspiels ist gegenüber der Wirklichkeit stark vereinfacht, so dass nicht in jedem Fall ein direkter Rückschluss auf das tatsächliche Verhalten von Wirtschaftssubjekten in der Realität gezogen werden kann. Die Versuchsanordnung ist jedoch derart gestaltbar, dass gerade durch die Abstraktion Einflüsse und deren Wirkung getrennt von fremden Einflussfaktoren zu beobachten sind. Die Aufgabe der experimentellen Wirtschaftsforschung besteht nun darin, aufgrund der gewonnenen Ergebnisse „die Theorie menschlichen Entscheidungsverhaltens deutlicher herauszuarbeiten“303. Danach kann in einem weiteren Schritt die Theorie auf die Wirklichkeit angewendet werden.

Bezogen auf die vorliegende Untersuchung bietet der Einsatz einer Unternehmenssimulation zur Datenerhebung einige Vorteile:

- Im Gegensatz zur Realität lassen sich bei einem Unternehmensplanspiel für alle simulierten Unternehmen identische Rahmenbedingungen in Bezug auf die Ausgangssituation, die Umfeldentwicklung und den Informationsstand

\footnotetext{
298 Synonym werden im Folgenden auch die Begriffe Unternehmenssimulation, Unternehmensspiel, Planspiel oder Management Game verwendet.

299 HögSDAL, 1996, S. 12.

300 Das erste Unternehmensspiel "Top Management Decision Simulation" ist in einer Publikation der American Management Association von RICCIARDI/CRAFT und anderen im Jahre 1957 beschrieben worden. Vgl. SAUERMANN/SELTEN, 1967, S. 6.

$301 \mathrm{Vgl}$. SAUERMANN/Selten, 1967, S. 6.

$302 \mathrm{Vgl}$. SaUermann/SeLTEN, 1967, S. 8.

303 SAUERMANN, 1970, S. 4.
} 
schaffen. Hinzu kommt die Branchenunabhängigkeit, da alle simulierten Unternehmen in der gleichen, mehr oder weniger genau definierten Branche tätig sind. Durch die Verwendung einer Unternehmenssimulation kann demnach eine Vergleichbarkeit des Datenmaterials erreicht werden.

- Die inhaltlichen Schwerpunkte, die Versuchsanordnung und der Ablauf sind im Vorfeld detailliert planbar, so dass gezielt Ursache-Wirkungs-Zusammenhänge ohne Störgrößen beobachtet werden können.

- Anders als in der Realität haben simulierte Unternehmen je nach Versuchsanordnung keine Möglichkeit, „sensible“ Informationen zurückzuhalten. In einem kontrollierten Experiment lässt sich sicherstellen, dass der Zugriff auf alle untersuchungsrelevanten Daten vollständig gegeben ist.

- Ein Unternehmensplanspiel ist in der Lage, eine dynamische Wettbewerbssituation über einen längeren Zeitraum zu simulieren. Den Teilnehmern wird dadurch ein derart komplexes Wettbewerbsumfeld vorgegeben, dass die annähernde Abbildung der zu untersuchenden Problemstellung möglich wird.

- Statistisch auswertbares Datenmaterial lässt sich im vorliegenden Fall aus Gründen, die bereits dargestellt wurden, nur begrenzt auf der Basis von real existierenden Unternehmen generieren. Bei Verwendung eines Unternehmensplanspiels erhält man vergleichbares Datenmaterial, das nach gleichen Rahmenbedingungen und in einer genügend großen Quantität ermittelt werden kann.

Durch den experimentellen Einsatz einer Unternehmenssimulation im Rahmen der vorliegenden Studie können die geschilderten Schwierigkeiten bei der Erhebung von Datenmaterial aus realen Unternehmen vermieden werden. Dies bedeutet keinen Realitätsverlust, da weiterhin reale Entscheidungen und ihre Auswirkungen analysiert werden. Gerade durch die abstrahierte Modellsituation können fremde Einflüsse ausgeschlossen und die Vergleichbarkeit und statistische Auswertbarkeit des Datenmaterials gewährleistet werden.

Zusammengefasst und auf der Basis der zuvor erläuterten Argumente lässt sich die Systematik des Entscheidungsprozesses schematisch in Abbildung 22 folgendermaßen darstellen: 


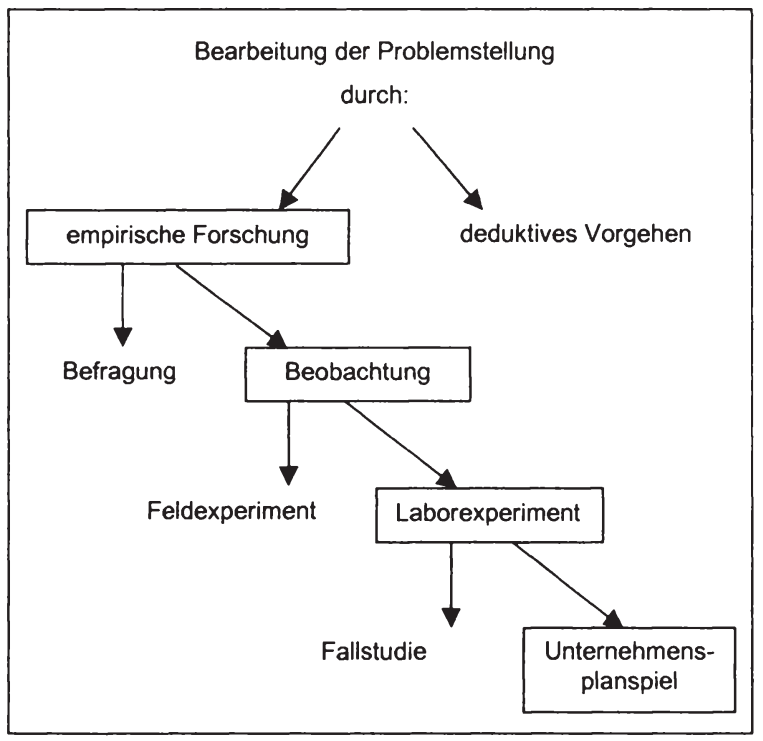

Abbildung 22: Der Entscheidungsprozess zum experimentellen Einsatz eines Unternehmensplanspiels in der vorliegenden Arbeit

Unter Abwägung der genannten Argumente sollen die zu betrachtenden Zusammenhänge mit Hilfe eines Unternehmensplanspiels untersucht werden. 


\section{Kapitel General Management Planspiel MARGA}

Die realitätsbezogene Überprüfung der im Teil I hergeleiteten Fragestellung soll, wie im 6. Kapitel erläutert wurde, anhand eines Unternehmensplanspiels durchgeführt werden. Einerseits verlangt es die Komplexität der Problemstellung, andererseits erlaubt es die Vielzahl der existierenden Unternehmenssimulationen, bei der Auswahl eines Planspiels strenge Kriterien anzulegen, die im Folgenden aufgestellt werden. Die Auswahl des General Management Planspiels MARGA wird im zweiten Abschnitt dieses Kapitels begründet, wobei in diesem Zuge MARGA ausführlich vorgestellt wird. Im dritten Abschnitt wird die intensive Wettbewerbssituation erläutert, in der sich die Planspiel-Unternehmen befinden und die dazu führt, dass bei MARGA der Konkurrenzanalyse ein großer Stellenwert zukommt. Aus diesem Grund und wegen der zentralen Bedeutung der Konkurrenzanalyse für die durchzuführende empirische Studie werden die Informationsquellen und die Instrumente, die im Rahmen der Konkurrenzanalyse bei MARGA einsetzbar sind, im vierten Abschnitt dargestellt.

\section{II.7.1 Auswahlkriterien für den experimentellen Planspieleinsatz im Rahmen der vorliegenden Studie}

Das auszuwählende Planspiel dient in dieser Arbeit als Hilfsmittel zur realitätsnahen Überprüfung der zu untersuchenden Fragestellungen. Inhaltlich stehen dabei die im 5. Kapitel dargestellte Problematik und die dort hergeleitete Grundhypothese im Mittelpunkt, nach der die Kenntnis der Wettbewerbsstärken und schwächen auf Kostenebene strategische Entscheidungen und damit den wirtschaftlichen Unternehmenserfolg beeinflusst.

Unternehmensplanspiele, die im 6. Kapitel als modellhafte Abbildungen von Unternehmen definiert wurden, lassen sich anhand einiger Merkmale unterschiedlich klassifizieren, wobei hier folgende Merkmale bei der Auswahl des Planspiels relevant sein werden: 304

304 Für weitere Klassifizierungsmerkmale vgl. HöGSDAL, 1996, S. 15. 


\section{Inhaltliche Ausrichtung:}

Planspiele werden in unterschiedlichen Disziplinen, wie bspw. im militärischen Bereich, in den Naturwissenschaften oder den Wirtschaftswissenschaften eingesetzt. Es liegt wegen des inhaltlichen Schwerpunktes der Fragestellung nahe, ein betriebswirtschaftliches Planspiel, genauer ein Unternehmensplanspiel auszuwählen.

Neben funktionsspezifischen Unternehmensplanspielen, die bspw. den Marketing- oder Logistik-Bereich eines Unternehmens fokussieren, bilden Allgemeine Planspiele, die auch General Management Planspiele genannt werden, das gesamte Entscheidungsgefüge eines Unternehmens ab. ${ }^{305} \mathrm{Da}$ bei haben die Planspiel-Teilnehmer ein Gesamtunternehmen zu leiten, bei dem alle Unternehmensbereiche eng miteinander verzahnt sind. Entscheidungen haben damit meist Auswirkungen auf andere Bereiche des Unternehmens. Weil die aufgeworfenen Fragestellungen die Gesamtunternehmensebene betreffen, ist für die Untersuchung ein General Management Planspiel auszuwählen.

Um auszuschließen, dass einzelne Versuchspersonen aufgrund vorhandener Branchenkenntnisse Vorteile gegenüber anderen Teilnehmern erlangen, sollte ein branchenneutrales Unternehmensplanspiel verwendet werden. ${ }^{306}$ Produkte und Märkte sind dort abstrakt beschrieben und nicht auf eine konkrete Wettbewerbssituation übertragbar.

\section{Komplexität:}

Die Komplexität eines Planspiels ist abhängig von der Anzahl der Entscheidungen, die in einer Periode gefällt werden und vom Vernetzungsgrad dieser Entscheidungen. Während die Anzahl der Entscheidungen leicht festzustellen ist - das Unternehmensplanspiel "Multi-Management-Simulation" weist 208 Entscheidungen je Periode auf 307 - liegen kaum Informationen über den Verknüftungsgrad vor. Da alle Entscheidungen, die je Periode zu fällen sind, in die Simulation einfließen und miteinander verknüpft

$305 \mathrm{Vgl.} \mathrm{GRAF,} \mathrm{1992,} \mathrm{S.} 134$.

306 Vgl. GRÜTtER-SETTELE, 1999, S. 78.

307 Vgl. GRAF, 1992, S. $101 \mathrm{ff}$. 
sind, kann davon ausgegangen werden, dass die Anzahl der Entscheidungen ein aussagekräftiges Merkmal der Komplexität darstellt.

Die komplexen Zusammenhänge, die in der Grundhypothese formuliert sind und die im Rahmen der vorliegenden Studie untersucht werden sollen, lassen sich nur durch einen entsprechend hohen Komplexitätsgrad im Unternehmensplanspiel abbilden. ${ }^{308}$ Dem entgegen steht die sinkende Motivation der Planspielteilnehmer, sich mit viel Zeitaufwand in hochkomplexe Simulationsmodelle einzuarbeiten. ${ }^{309}$ Es ist folglich ein Unternehmensplanspiel auszuwählen, das unter Berücksichtigung des Zielkonfliktes zwischen Komplexität und Motivation aussagekräftige Untersuchungsergebnisse erwarten lässt. Auf der Grundlage dieser Vorüberlegungen wird im Rahmen der Untersuchung der Einsatz einer Unternehmenssimulation mit 70 bis 90 Entscheidungen je Periode als sinnvoll erachtet. ${ }^{310}$

\section{Konkurrenzsituation:}

Grundsätzlich können Unternehmensplanspiele in zwei Konkurrenzsituationen eingeteilt werden. Bei einer starren oder statischen Konkurrenzsituation spielt ein Planspiel-Unternehmen in einem vorher festgelegten Wettbewerbsszenario gegen Konkurrenten, die von einer Software über alle Perioden hinweg simuliert werden. Versuchspersonen treten in Form einer Mensch-Maschine Interaktion gegen ein starres Modell an. Dies hat zur Folge, dass es bestimmte, vorher feststehende Entscheidungen gibt, die über den Spielverlauf hinweg zu einem „besten“ Spielergebnis, bspw. zur Gewinnmaximierung führen. Dieser "optimale Entscheidungsmix" wird in dem Moment fixiert, in dem die Planspielleitung das Wettbewerbsszenario und das Verhalten der simulierten Konkurrenten festlegt. Ein Unternehmensplanspiel mit statischer Konkurrenzsituation wird daher immer von dem Team gewonnen, das dem „optimalen Entscheidungsmix“ am nächsten kommt. Simulationsmodelle mit statischer Konkurrenzsituation stellen damit für die Teilnehmer letztlich ein rein mathematisches Optimierungsproblem dar.

308 Ähnlich: GRÜtTER-SETTELE, 1999, S. 77.

309 Diese Einschätzung beruht auf der beruflichen Erfahrung des Autors.

310 Diese Einschätzung beruht auf der beruflichen Erfahrung des Autors. Ähnlich: GRÜTTERSeTtele, 1999, S. $79 \mathrm{f}$. 
Eine dynamische Konkurrenzsituation wird in Simulationsmodellen abgebildet, bei denen konkurrierende Unternehmen durch natürliche Personen gesteuert werden. Die Planspiel-Unternehmen stehen dabei in einer direkten Wettbewerbssituation zueinander und beeinflussen sich gegenseitig. Gegenspieler sind nicht mehr simulierte Konkurrenten, deren Verhalten im Vorfeld von der Planspielleitung definiert worden ist, sondern andere Teilnehmer mit eigenen individuellen Strategien. Die Gewinnmaximierung stellt damit kein vorher festgelegtes mathematisches Optimierungsproblem dar, sondern hängt davon $a b$, welches Verhalten die Wettbewerber zeigen und wie das einzelne Unternehmen in diesem dynamischen Umfeld agiert und reagiert.

In der vorliegenden Studie, in der u.a. menschliches Entscheidungsverhalten in Abhängigkeit von bestimmten Informationen untersucht wird, liegt daher die Verwendung eines Planspiels mit dynamischer Konkurrenzsituation nahe. ${ }^{311}$ Unternehmenssimulationen mit starrer Konkurrenzsituation kommen demnach nicht in Betracht.

\section{Zeithorizont:}

Die Auswirkungen strategischer Entscheidungen sind langfristig, was im Rahmen dieser Untersuchung eine entsprechende Anforderung an das Unternehmensplanspiel stellt: Es sollten mindestens vier bis fünf aufeinander aufbauende Perioden simuliert werden. Das Simulationsmodell muss dabei so beschaffen sein, dass die Auswirkungen strategischer Entscheidungen, die in der Mitte des zeitlichen Ablaufs getroffen werden, noch vor dem Ende des Planspiels sichtbar werden. ${ }^{312}$

\section{Einsatzform:}

Unternehmensplanspiele werden im Rahmen von Seminaren oder als Fernplanspiel eingesetzt. Ein Fernplanspiel wird, im Gegensatz zum Seminarplanspiel, über eine beliebige räumliche Distanz durchgeführt. Dabei erhalten die Teilnehmer alle notwendigen Unterlagen per Post oder auf elektronischem Wege zugeschickt. Die Teilnehmer arbeiten sich selbstständig in das Regelwerk des Planspiels ein und senden die Entscheidungen zu vor-

311 Ähnlich: GrütTER-SetTele, 1999, S. 77.

312 Diese Einschätzung beruht auf der beruflichen Erfahrung des Autors. 
gegebenen Zeitpunkten per e-mail, Fax oder Brief an die Planspielleitung. Diese startet nach Erhalt der Daten die Simulation der Märkte und sendet daraufhin die Ergebnisse an die Teilnehmer zurück.

Im Rahmen einer empirischen Untersuchung hat diese Einsatzform einen entscheidenden Vorteil: Nur durch ein Fernplanspiel können die Entscheidungen von mehreren hundert Unternehmen mit insgesamt mehreren tausend Teilnehmern gleichzeitig am selben Simulationsmodell und unter identischen Rahmenbedingungen untersucht werden. Dieser Umstand ist eine notwendige Voraussetzung für die Vergleichbarkeit der Entscheidungsdaten.

Darüber hinaus ist zu beachten, dass die Aussagekraft einer empirischen Untersuchung mit zunehmender Größe der Grundgesamtheit steigt. Erst wenn eine kritische Größe der Grundgesamtheit überschritten ist, lassen sich die erfassten Daten mit Hilfe mathematisch-statistischer Verfahren analysieren. Da eine empirische Untersuchung auf der Basis von Seminaren, in denen ein entsprechendes Planspiel zum Einsatz kommt, sehr kosten- und zeitintensiv is ${ }^{313}$, weist der Seminareinsatz für diese Untersuchung große Nachteile und Risiken auf, die durch den Einsatz eines Fernplanspiels vermieden werden können.

Einen weiteren Aspekt stellt die Kontaktmöglichkeit der konkurrierenden Teilnehmer dar. Im Seminar können alle Wettbewerber unkontrollierbar miteinander kommunizieren. Absprachen, die das Verhalten aller oder einzelner Teams beeinflussen, sind damit nicht auszuschließen. Dem gegenüber sind die Kommunikationsmöglichkeiten im Fernplanspiel von der Planspielleitung vollständig steuerbar. Die Planspielleitung bestimmt, zu welchem Zeitpunkt welche Planspiel-Unternehmen welche Informationen erhalten. Für die vorliegende Untersuchung ist eine einheitliche Informationsbasis der teilnehmenden Teams von entscheidender Bedeutung, wie in den späteren Ausführungen noch zu erläutern ist. Daher spricht auch dieser Aspekt gegen die Verwendung eines Seminar- und für den Einsatz eines Fernplanspiels.

313 Vgl. GRÜTTER-SETTELE, 1999, S. 81. 
Die jeweilige Einsatzform setzt bestimmte technische und organisatorische Eigenschaften des Planspiels voraus, so dass Fernplanspiele normalerweise auch für den Seminareinsatz geeignet sind, jedoch nur wenige Seminarplanspiele als Fernplanspiel eingesetzt werden können. Erschwerend kommt hinzu, dass nur einige wenige Fernplanspiele mehrere hundert Unternehmen gleichzeitig simulieren können.

\section{Motivation der Teilnehmer:}

Der erfolgreiche Einsatz von Unternehmenssimulationen im Rahmen von empirischen Studien ist an die Leistungsbereitschaft der Versuchspersonen gebunden. Die Grundvoraussetzung für persönliches Engagement der Teilnehmer ist die Akzeptanz des Planspiels, die maßgeblich von einigen Faktoren abhängt:

- Das Planspiel muss technisch und organisatorisch zuverlässig funktionieren.

- Die inhaltlichen Zusammenhänge eines Planspiels müssen korrekt dargestellt und simuliert sein. Ökonomisch unsinnige Entscheidungen der Teilnehmer müssen zu einem Misserfolg führen.

- Die Marktsimulation mit all ihren einzelnen Ergebnissen muss für die Teilnehmer nachvollziehbar und in sich schlüssig sein.

- Das auszuwählende Planspiel darf in den Augen der Teilnehmer kein Glücksspiel sein, bei dem zufällige Entwicklungen den eigenen Erfolg beeinflussen.

- Die Einarbeitung in das Regelwerk des Planspiels darf nicht zu viel Zeit beanspruchen. Im Übrigen kann die empirische Studie erst dann beginnen, wenn alle Teilnehmer das Regelwerk beherrschen und überlegte Entscheidungen fällen können.

Diese Faktoren, die die Akzeptanz und die Leistungsbereitschaft der Teilnehmer bestimmen, scheinen selbstverständlich zu sein. In der Realität können jedoch selbst renommierte Planspielanbieter einige Faktoren, wie bspw. die Fehlerfreiheit, nur bis zu einem gewissen Grad optimieren und nie vollständig erfüllen. Hier haben erprobte Planspiele deutliche Vorteile gegenüber neu entwickelten Planspielen. 
Der aufgestellte Kriterienkatalog, mit dessen Hilfe Planspiele für den experimentellen Einsatz in empirischen Studien ausgewählt werden können, ist sicherlich über die vorliegende Untersuchung hinaus anwendbar. Die dabei angestellten Überlegungen sollen auch hier die Grundlage für die Auswahl eines geeigneten Planspiels sein.

\section{II.7.2 Auswahl des General Management Planspiels MARGA}

Die Auswahl eines geeigneten Planspiels für die vorliegende Untersuchung beschränkt sich auf den deutschen Planspielmarkt. Mit ca. 1.500 Planspielen ${ }^{314}$ wird hier bereits eine Vielfalt geboten, die eine sinnvolle Auswahl ermöglichen sollte. Die im vorherigen Abschnitt genannten Klassifizierungsmöglichkeiten von Planspielen und die daraus abgeleiteten Auswahlkriterien für den experimentellen Einsatz im Rahmen dieser empirischen Untersuchung schränken den Kreis der in Frage kommenden Simulationsmodelle stark ein. Folgende zwei Unternehmenssimulationen zählen am deutschen Planspielmarkt u.a. zu den etablierten Produkten und kommen in eine engere Auswahl:315

1. MARGA,

2. TOPSIM-General-Management.

In den folgenden Ausführungen werden die zwei Simulationen, die sich in einigen Aspekten voneinander unterscheiden, auf der Basis des zuvor aufgestellten Kriterienkataloges bewertet. Da zum Zeitpunkt dieser Studie, wie noch zu zeigen sein wird, ausschließlich das General Management Planspiel MARGA alle aufgestellten Auswahlkriterien erfüllt, wird dieses im Rahmen der Kriterienüberprüfung gleichzeitig ausführlicher vorgestellt, während TOPSIMGeneral-Management nur im Hinblick auf den Kriterienkatalog bewertet wird.

314 Vgl. ROHN, 1992.

315 In einer Vorauswahl zählt GRÜTTER-SETTELE insgesamt acht Unternehmenssimulationen auf, von denen jedoch sechs aus diversen Gründen, auf die an dieser Stelle nur verwiesen werden soll, ausscheiden. Letztlich entscheidet sich GRÜTTER-SETTELE ebenfalls für die Planspiele MARGA und TOPSIM-General-Management, die in eine engere Auswahl kommen. Vgl. GRÜTTER-SETTELE, 1999, S. $79 \mathrm{ff}$. 
Das General Management Planspiel MARGA 316 ist eine Initiative des USW, UNIVERSITÄTSSEMINAR DER WIRTSCHAFT, SCHLOSS GRACHT, und der VERLAGSGRUPPE HANDELSBLATT GMBH. MARGA wird seit 1971 überwiegend in der Personalentwicklung als ein Instrument der Aus- und Weiterbildung, aber auch zu Forschungszwecken ${ }^{317}$ eingesetzt. Während MARGA sich vornehmlich an Führungs- und Nachwuchskräfte aus Unternehmen wendet, führt das USW seit 1998 zusammen mit der MANNESMANN AG zusätzlich das Unternehmensplanspiel für Studenten THE BOARD durch. THE BOARD basiert auf dem selben Simulationsmodell wie MARGA. Beide Planspiele sind zum Zeitpunkt der Untersuchung völlig identisch, wobei aufgrund des Unterschiedes in der Zielgruppe eine getrennte Betrachtung erfolgt. ${ }^{318}$

\section{Inhaltliche Ausrichtung von MARGA:}

Das MARGA-Modell simuliert ein Gesamtunternehmen mit den Bereichen Marketing, Produktion, Einkauf, Logistik, Personal und Finanzen. ${ }^{319}$ Alle Unternehmensbereiche sind eng miteinander verbunden und von den Teilnehmern mit insgesamt 77 Einzelentscheidungen zu steuern. Es gilt, auf vier Märkten, Europa, Russland, den USA und Japan, drei Produkte anzubieten. Die Produkte sind nur abstrakt beschrieben, so dass das MARGA-Unternehmen keiner Branche zuzurechnen ist. Die Produkte weisen in der bei dieser Studie zum Einsatz kommenden Ausgangssituation sehr unterschiedliche Merkmale auf:

- Das Produkt 1 stellt ein Konsumgut dar, dessen Zielgruppe der Endverbraucher ist. Es erwirtschaftet pro Stück eine relativ geringe Gewinnmarge, wird jedoch in großen Stückzahlen produziert, so dass ein Umsatzanteil von $45 \%$ erzielt wird. Das Produkt 1 befindet sich in der Reifephase des Produktlebenszyklusses ${ }^{320}$ und ist damit das älteste der drei MARGA-Produkte.

316 Der geschützte Markenname MARGA steht für MARket GAme.

317 Vgl. Franzen, 1985; GRÜTTER-SetTEle, 1999.

318 Da THE BOARD von MARGA abgeleitet ist, wird der Einfachheit halber im Folgenden meist nur MARGA erwähnt, obwohl beide Planspiele gemeint sind.

319 Das Regelwerk des MARGA Simulationsmodells soll im Rahmen dieser Untersuchung aus Platzgründen nicht vollständig dargestellt werden. Es wird in diesem Zusammenhang auf das MARGA-Handbuch verwiesen. VgI. UNIVERSITÄTSSEMINAR DER WIRTSCHAFT, MARGAHandbuch 1999.

320 Das Konzept des Produktlebenszyklusses soll im Rahmen dieser Arbeit nicht näher dargestellt werden. Es wird auf die Ausführungen von WELGE/AL-LAHAM und auf die dort angeführte Literatur verwiesen. Vgl. WELGE/AL-LAHAM, 1999, S. $233 \mathrm{ff}$. 
- Das Produkt 2 ist eine neu entwickelte Dienstleistung, die sich im Produktlebenszyklus noch in der Einführungsphase befindet. Das Dienstleistungsprodukt ist sehr personalintensiv und weist eine hohe Gewinnmarge pro Einheit auf. Der Umsatzanteil liegt bei $10 \%$.

- Das Produkt 3 ist ein technisch hoch entwickeltes Investitionsgut. Es befindet sich bezogen auf den Produktlebenszyklus in der Wachstumsphase, ist jedoch recht konjunkturempfindlich, was es aufgrund der Umfelddaten von MARGA '99 auf einigen Märkten wirtschaftlich attraktiv und auf anderen Märkten unattraktiv macht. Der Umsatzanteil beträgt $45 \%$.

Die drei Produkte weisen diverse unterschiedliche Merkmale auf, was für die vorliegende Studie positiv bewertet wird, weil auf diese Weise ein breiter Durchschnitt verschiedener Branchen-, Produkt- und Marktbesonderheiten simuliert werden kann, was zu einer größeren Allgemeingültigkeit der Untersuchungsergebnisse führt.

Inhaltlich ist MARGA als betriebswirtschaftliches Planspiel einzuordnen, bei dem das gesamte Entscheidungsgefüge eines Unternehmens simuliert wird. MARGA wird daher auch als General Management Planspiel bezeichnet.

TOPSIM-General-Management simuliert - wie MARGA - ein gesamtes Unternehmen mit mehreren Bereichen und Abteilungen, die eng verzahnt sind und von den Teilnehmern ökonomisch sinnvoll aufeinander abgestimmt werden müssen. Im Unterschied zu MARGA, das branchenneutral ist, wird bei TOPSIM ein Kopiergerätehersteller abgebildet. Wie im Kriterienkatalog erläutert, wird für die vorliegende Untersuchung jedoch ein branchenneutrales Planspiel bevorzugt.

\section{Komplexität von MARGA:}

Mit 77 einzelnen Entscheidungen je Periode und einer vergleichsweise engen Verzahnung dieser Entscheidungen ist die Komplexität des MARGA-Modells als hoch zu bezeichnen. Besonders die starke Verflechtung der Variablen wird für eine empirische Untersuchung als problematisch gewertet, weil einige zu zeigende Ursache-Wirkungs-Zusammenhänge in der Ursprungsversion nicht zweifelsfrei abgeleitet werden können. Diese Problematik kann nur durch eine entsprechende Versuchsanordnung umgangen werden, die bspw. punktuell bestimmte Verflechtungen auflöst oder die Wirkung von Ursachen anhand von 
planspielunabhängigen Instrumenten misst. Auf diese Weise können trotz der engen Verzahnung der 77 MARGA-Entscheidungen zuverlässige Aussagen über die zu untersuchenden Zusammenhänge abgeleitet werden. ${ }^{321}$

In einer ausreichend intensiven Testphase haben alle Teams die Gelegenheit, das komplexe Regelwerk des Planspiels kennen zu lernen, so dass in der sich anschließenden Hauptrunde, in der das Datenmaterial erhoben werden soll, mit aussagekräftigen Untersuchungsergebnissen gerechnet werden kann.

Die Komplexität von TOPSIM ist mit ca. 70 Entscheidungen vergleichbar mit der von MARGA.

\section{Konkurrenzsituation bei MARGA:}

Die MARGA-Teilnehmer befinden sich in einer dynamischen Konkurrenzsituation. Es stehen ausschließlich mit Teilnehmern besetzte Teams im Wettbewerb zueinander, die in Gruppen zu jeweils vier Unternehmen eingeteilt sind. Jedes Unternehmen beeinflusst mit seinem Handeln nicht nur den eigenen, sondern auch den Erfolg der drei konkurrierenden Unternehmen. Bspw. führt ein aggressives Marketing dazu, dass das entsprechende Unternehmen seinen Marktanteil vergrößern kann, während die Wettbewerber Marktanteile verlieren. Mit seinem eigenen Verhalten provoziert man gleichzeitig eine entsprechende Reaktion der Konkurrenten, die in der Folge auf die Strategie des Ersten antworten.

Das MARGA-Simulationsmodell basiert damit in erster Linie auf menschlichen Entscheidungen, so dass MARGA zur Untersuchung der vorliegenden Fragestellung geeignet ist.

TOPSIM bietet ein gleichwertiges dynamisches Konkurrenzmodell und ist damit unter diesem Aspekt ebenfalls für die Untersuchung geeignet.

\section{Zeithorizont bei MARGA:}

MARGA wird über sechs, THE BOARD über fünf aufeinander folgende Perioden gespielt, wobei eine Periode einem Quartal entspricht. Nach jedem Quar-

321 Die Versuchsanordnung der vorliegenden empirischen Untersuchung wird im nachfolgenden 8. Kapitel ausführlich dargestellt. 
tal erhalten die Teilnehmer einen vollständigen Überblick über das interne und externe Rechnungswesen ihres Unternehmens, so dass in jeder Periode auf der Basis aktueller Informationen gesteuert werden kann. Das Simulationsmodell reagiert auf strategische Entscheidungen, die für die Umsetzung von Strategien relevant sind, mit maximal einer Periode Verzögerung. ${ }^{322}$ Die Auswirkungen einer Entscheidung sind daher spätestens in der Folgeperiode zu sehen. Damit wirken sich strategische Entscheidungen bis zum Ende des Planspiels bei MARGA noch in der 5 . und bei THE BOARD noch in der 4 . Periode aus. Der simulierte Zeithorizont reicht somit bei beiden Planspielen für eine Analyse strategischer Entscheidungen aus. Da der Zeithorizont bei MARGA und bei THE BOARD jedoch unterschiedlich ist, erfolgt die Betrachtung getrennt voneinander.

Das ebenfalls zur Auswahl stehende Planspiel TOPSIM-General-Management ist in Bezug auf die zeitliche Umsetzbarkeit strategischer Entscheidungen mit MARGA vergleichbar. Da bei TOPSIM jedoch wahlweise insgesamt acht Perioden simuliert werden können, was das MARGA-Modell nicht erlaubt, ergibt sich hier ein Vorteil, der für TOPSIM spricht, weil Unternehmensstrategien über einen längeren Zeitraum durchgeführt werden können. Der Einsatz von TOPSIM wäre demnach unter diesem Aspekt zu bevorzugen, wobei auch MARGA die grundsätzlichen Voraussetzungen erfültt.

\section{Einsatzform von MARGA:}

MARGA und THE BOARD werden als Fernplanspiel im Rahmen eines Wettbewerbs eingesetzt, der offen ausgeschrieben wird. Interessenten können sich nur als Team mit maximal sechs Teammitgliedern beteiligen, wobei eine durchschnittliche Teamgröße von über fünf Personen angenommen werden kann. ${ }^{323}$ Beide Planspiel-Wettbewerbe werden nur einmal jährlich durchgeführt und starten jeweils im Herbst.

322 Im Gegensatz zu strategischen Entscheidungen sind operative Entscheidungen zu sehen, die eher für die taktisch-kurzfristige Steuerung des Planspiel-Unternehmens eingesetzt werden.

323 Da die Teilnahmegebühr pro Team zu entrichten ist, melden sich meist sechsköpfige Teams an, um ein günstiges Preis-Leistungsverhältnis pro Kopf zu erzielen. Da dies nicht in allen Fällen gelingt, wird die durchschnittliche Teamgröße vom Verfasser auf der Basis von stichprobenartigen Befragungen auf über fünf, aber unter sechs geschätzt. 
Für die vorliegende Studie kamen die Fernplanspiel-Wettbewerbe MARGA '99 und THE BOARD '99 in Betracht, die beide im Herbst 1998 begannen und im Sommer 1999 endeten. An MARGA '99 haben sich insgesamt 284 Teams, d.h. ca. 1.500 Führungskräfte beteiligt. An THE BOARD '99 haben 472 Teams mit ca. 2.500 Studenten teilgenommen. Eine empirische Untersuchung auf der Basis eines Unternehmensplanspiels, mit einer Grundgesamtheit von insgesamt 756 Teams und ca. 4.000 beteiligten Personen, aufgeteilt in Führungskräfte und Studenten, wobei alle Planspiel-Unternehmen nach der selben Version des selben Simulationsmodells und unter identischen Rahmenbedingungen das Experiment durchlaufen, ist nur mit Hilfe eines Fernplanspiels durchführbar. MARGA ist auf dem deutschsprachigen Planspielmarkt seit 1971, dem Jahr der ersten Durchführung, marktführend bei Fernplanspielen mit dynamischer Konkurrenzsituation. Das Gleiche gilt für THE BOARD, das jedoch erstmalig 1998/ '99 für Studenten durchgeführt worden ist.

Im Gegensatz zu MARGA ist TOPSIM-General-Management ein überwiegend in Seminaren eingesetztes Planspiel. Zwar wurde diese Simulation zum Zeitpunkt der Untersuchung auch als Fernplanspiel eingesetzt, jedoch in einem deutlich geringeren Umfang, der in der Größenordnung mit den offen ausgeschriebenen Fernplanspiel-Wettbewerben MARGA und THE BOARD nicht vergleichbar ist.

\section{Motivation der Teilnehmer:}

Die im vorherigen Abschnitt II.7.1 aufgeführten Faktoren, die die grundsätzliche Leistungsbereitschaft der Versuchsteilnehmer beeinflussen, können beim MARGA-Modell als erfüllt angesehen werden. Das MARGA-Modell wird seit Beginn einer permanenten technischen, organisatorischen und inhaltlichen Überarbeitung unterzogen. Gepaart mit einer hohen Teilnehmerzahl im Fernplanspiel - seit 1971 kann die Zahl der Führungskräfte, die an MARGA teilgenommen haben auf deutlich über 50.000 geschätzt werden - führt dies zu einer Fehlerminimierung und $\mathrm{zu}$ einer maximalen Zuverlässigkeit. Diese Faktoren sind von grundlegender Bedeutung für die Akzeptanz des Planspiels, für die Leistungsbereitschaft der Teilnehmer und damit für die Aussagekraft der empirischen Untersuchung. 
Die Motivation der Versuchspersonen lässt sich durch extrinsische und intrinsische Faktoren beeinflussen. ${ }^{324}$ Intrinsische Motivation liegt dann vor, wenn die Motivation ihre Befriedigung in der Tätigkeit selbst findet, d.h. wenn die Teilnehmer eine Befriedigung von individuellen Bedürfnissen dadurch erreichen, dass sie am Planspiel teilnehmen. Der Wunsch, durch das Planspiel etwas zu lernen, ist hier als Beispiel zu nennen. Extrinsische Motivation erwächst aus Folgen oder Begleitumständen einer Tätigkeit, hier der Planspielteilnahme. Als Beispiel seien monetäre Anreize genannt. ${ }^{325}$ Sowohl bei MARGA, als auch bei THE BOARD sind diverse intrinsische und extrinsische Faktoren zu nennen.

Intrinsische Motivationsfaktoren:

- Das Bedürfnis, durch das Planspiel das eigene Wissen in vielen Themenbereichen, die bei MARGA und bei THE BOARD vermittelt werden, zu erweitern.

- Die Herausforderung, in einem Unternehmen eigene Strategien entwickeln und verwirklichen zu können.

- Der Reiz, sich im Rahmen eines offen ausgeschriebenen Wettbewerbs mit anderen Konkurrenten zu messen. Die Wettbewerbssituation bringt oft Dynamik und Teamgeist in die Gruppe.

Extrinsische Motivationsfaktoren:

- Die Honorierung guter Leistungen im Planspiel durch Vorgesetzte und Kollegen, was in erster Linie für MARGA gilt. Die den teilnehmenden Teams von Seiten ihres Unternehmens entgegengebrachte Anerkennung reicht von kleinen materiellen Zuwendungen, wie z.B. Buchpreisen, bis hin zu immateriellen Anreizen, wie einem positiven Vermerk in der Personalakte der Teilnehmer oder die Aufmerksamkeit der Unternehmensleitung auf engagierte und erfolgreiche Mitarbeiter.

- Die Berichterstattung über den Verlauf des Planspiel-Wettbewerbs in überregionalen Finanzzeitungen. ${ }^{326}$

- Der Wunsch, das dreitägige Finale in SCHLOSS GRACHT der besten acht Teams zu erreichen.

$324 \mathrm{Vgl}$. FRANZEN, 1985, S. $103 \mathrm{f}$. und S. $123 \mathrm{f}$.

325 Vgl. ROSENSTIEL, 1999, S. 179.

326 Vgl. HEINEN, 1999 (a), (b), (c), (d) und o.V., 1999. 
- Bei THE BOARD der Anreiz für Studenten, Kontakt zu einem potentiellen späteren Arbeitgeber, in diesem Fall dem MANNESMANN-Konzern, zu erlangen.

- Der attraktive finanzielle Anreiz für die Finalisten von THE BOARD in Form von Preisgeldern.

Die Motivation der Teilnehmer kann insgesamt als ausgesprochen hoch eingeschätzt werden, was sich bspw. darin äußert, dass sowohl MARGA als auch THE BOARD überwiegend in der persönlichen Freizeit der Teilnehmer gespielt werden.

TOPSIM-General-Management zählt in Deutschland zu den marktführenden Unternehmenssimulationen für den Seminareinsatz. Das Planspiel ist bezüglich der aufgestellten qualitativen Kriterien, die die Akzeptanz und damit die Leistungsbereitschaft der Teilnehmer in starkem Maße beeinflussen, mit MARGA vergleichbar.

Da MARGA und THE BOARD im Rahmen eines offenen Wettbewerbs durchgeführt werden, können einige der oben erwähnten intrinsischen und extrinsischen Motivationsfaktoren beim Seminareinsatz von TOPSIM nicht erreicht werden. Andererseits entsteht in Seminaren oftmals eine starke Dynamik, die sich positiv auf die Motivation der Teilnehmer auswirkt. 327 Folglich werden auch diesbezüglich TOPSIM und MARGA gleich bewertet.

Zusammengefasst und mit Hilfe eines Punktesystems, anhand dessen die Eignung von MARGA und TOPSIM für den Einsatz in der hier durchzuführenden empirischen Untersuchung bewertet wird, ergibt sich in Tabelle 3 ein Überblick. 328

327 Diese Einschätzung beruht auf der beruflichen Erfahrung des Autors als Dozent und Planspielleiter am USW, UNIVERSITÄTSSEMINAR DER WIRTSCHAFT, SCHLOSS GRACHT.

328 Für eine besonders gute Eignung werden fünf Punkte, für eine schlechte Eignung wird ein Punkt vergeben. Die Entscheidung fällt zu Gunsten des Planspiels aus, das insgesamt die meisten Punkte erhält. Der Bewertung liegen ausschließlich die aufgestellten Kriterien zu Grunde, die für die vorliegende Studie relevant sind. 


\begin{tabular}{|l|c|c|}
\hline Kriterien & MARGA & TOPSIM \\
\hline Inhaltliche Ausrichtung & 5 & 3 \\
\hline Komplexität & 5 & 5 \\
\hline Konkurrenzsituation & 5 & 5 \\
\hline Zeithorizont & 4 & 5 \\
\hline Einsatzform & 5 & 1 \\
\hline Motivation der Teilnehmer & 5 & 5 \\
\hline Summe der Punkte & 29 & 24 \\
\hline
\end{tabular}

Tabelle 3: Die Auswahl des Unternehmensplanspiels MARGA

Von 30 möglichen Punkten erhält MARGA 29 und TOPSIM 24 Punkte. Unter Berücksichtigung aller zuvor aufgestellten Kriterien, wird damit das General Management Planspiel MARGA für die vorliegende empirische Untersuchung ausgewählt, obwohl auch TOPSIM-General-Management allen inhaltlichen und qualitativen Ansprüchen gerecht wird. Ausschlaggebend für diese Entscheidung ist die Einsatzform der Simulation. Im Rahmen des für diese Studie durchzuführenden Laborexperimentes bringt der Einsatz in Seminaren, wie zuvor bereits dargelegt wurde, zu viele Nachteile mit sich, während der Fernplanspieleinsatz hier deutlich vorteilhafter ist.

Neben den bereits genannten Gründen sprechen in Anlehnung an GRÜTTERSETTELE zwei weitere für die Verwendung des MARGA-Fernplanspiels: ${ }^{329}$

- 1985 untersuchte FRANZEN mit Hilfe einer Vorgängerversion von MARGA den Informationsnutzen einer Voll-, bzw. einer Teilkostenrechnung. ${ }^{330}$ 1996/ '97 führte GRÜTTER-SETTELE auf der Basis des MARGA-Modells eine Studie zur Verhaltenswirkung von Informationen des externen Rechnungswesens durch. 331 Die dort gesammelten Erfahrungen bezüglich der methodischen Gestaltung und Strukturierung der Untersuchung können auch im vorliegenden Fall genutzt werden.

- Wie franzen und Grütter-Settele zuvor, leitet der Verfasser der vorliegenden Studie seit 1997 das Planspiel MARGA. Insofern konnte sichergestellt werden, dass der Versuchsaufbau vom Verfasser den Bedürfnissen

329 Vgl. GRÜTter-Settele, 1999, S. 82.

$330 \mathrm{Vgl.} \mathrm{FRANZEN,} 1985$.

331 Vgl. GRÜTtER-SETtEle, 1999. 
gerecht konzipiert wurde, die Versuchsdurchführung sorgfältig geprüft wurde und alle untersuchungsrelevanten Daten korrekt erfasst wurden.

Zusammenfassend ist zu sagen, dass ausschließlich das Unternehmensplanspiel MARGA zum Zeitpunkt der vorliegenden Untersuchung die aufgestellten Auswahlkriterien in diesem hohen Maße erfüllen kann.

\subsubsection{Wettbewerbsumfeld und strategisches Verhalten der Planspiel- Unternehmen bei MARGA und THE BOARD}

Die vorliegende Studie untersucht, inwiefern die Kenntnis der Wettbewerbsstärken und -schwächen auf der Kostenebene strategische Entscheidungen und damit den wirtschaftlichen Erfolg beeinflussen. Es liegt nahe, nach der Auswahl des Planspiels zunächst die Wettbewerbssituation zu analysieren, in der sich die Planspiel-Unternehmen bewegen. Nach diesem Schritt werden mögliche strategische Verhaltensweisen der Teams abgeleitet.

Alle 756 Planspiel-Unternehmen starten mit der gleichen Ausgangssituation. Je vier Teams werden einer Gruppe, bzw. einer Branche 332 zugeordnet und stehen in direktem Wettbewerb zueinander. Da alle konkurrierenden MARGA-Unternehmen anfangs in den gleichen Marktsegmenten, mit den gleichen Produkten und der gleichen Ressourcenausstattung aufeinander treffen, ist mit einer hohen Wettbewerbsintensität zu rechnen. Diese verschärft sich noch dadurch, dass die simulierte Nachfrageseite jederzeit vollständig über das Angebot der vier MARGA-Unternehmen informiert ist, so dass hier von vollkommenem Wettbewerb ausgegangen werden kann. ${ }^{333}$

Der Wettbewerb bei MARGA besteht darin, dass auf der Angebotsseite vier Unternehmen gleicher Wertschöpfungsebene um eine begrenzte Nachfrage konkurrieren, so dass hier von einer horizontalen Konkurrenzsituation gesprochen werden kann. ${ }^{334}$ Da keine weiteren potentiellen Wettbewerber simu-

332 Die Begriffe Gruppe und Branche werden in diesem Zusammenhang synonym verwendet, wobei eine Branche hier als Gruppe von vier MARGA-Unternehmen zu sehen ist, "die Produkte herstellen, die sich gegenseitig nahezu ersetzen können“. PORTER, 1999 (a), S. 35.

333 Vgl. GRÜTTER-SETTELE, 1999, S. 77.

334 Vgl. 2. Kapitel, Abschnitt I.2.1. 
liert werden, hat jedes Planspiel-Unternehmen drei relevante Konkurrenten. ${ }^{335}$ Zwischen den MARGA-Teams ist weiterhin eine Ressourcen Konkurrenz um die Ressource "Facharbeiter" festzustellen, deren Bedeutung im Planspiel jedoch als gering einzuschätzen ist ${ }^{336}$ und die in der durchzuführenden Untersuchung keine Rolle spielt ${ }^{337}$. Da die Wertschöpfungstiefe von den Unternehmen nicht beeinflussbar ist, liegt keine vertikale Konkurrenzsituation vor. ${ }^{338}$

Während nur vier Unternehmen das gesamte Angebot in der jeweiligen Branche darstellen, wird die Nachfrage von einer nicht näher quantifizierten großen Anzahl von Kunden repräsentiert. Die Unternehmen sind damit Teil eines Oligopols, wo das eigene Handeln den Erfolg der Konkurrenten beeinflusst und Gegenreaktionen hervorruft. Jede Entscheidung muss daher unter Berücksichtigung möglicher Konkurrenzreaktionen getroffen werden.

Die vier in Konkurrenz zueinander stehenden Teams sind anonymisiert, d.h. kein Team weiß, hinter welchem Planspiel-Unternehmen welches Team steht und zu welchem realen Unternehmen oder zu welcher Hochschule die Teilnehmer gehören. Da die Teams durch eine ausführliche und für alle identische Konkurrenzanalyse 339 über das Verhalten inrer Wettbewerber laufend informiert sind, können sie folglich nur über ihr Verhalten im Planspiel miteinander kommunizieren. So kann sich bspw. ein Unternehmen durch seine Preispolitik kooperativ zeigen, indem es keine aggressiven Preissenkungen vornimmt. Auf diese Weise kann sich ein stabiles Preisniveau mit entsprechend hohen Gewinnmargen etablieren. Verstößt einer der Wettbewerber gegen diese „stillschweigende Kooperation“, hat dieser den Vorteil eines erhöhten Marktanteils. Ziehen die Konkurrenten nach, was wahrscheinlich ist, drohen Preiskämpfe mit entsprechend sinkenden Margen.

335 Die Begriffe der "potentiellen Wettbewerber" und der "relevanten Konkurrenten" stammen von PORTER bzW. von HAX/MAJLUF und werden im 2. Kapitel, Abschnitt 1.2.1, erläutert.

336 Folgende Gründe sind zu nennen: 1. Gewöhnlich kommt es - gemäß der beruflichen Erfahrung des Autors - nur in wenigen Vierergruppen zu einer unbefriedigten Nachfrage nach Facharbeitern und damit zu einer Situation der Knappheit; 2. Die Teams können selber Facharbeiter ausbilden und sind so in der Lage, den Mangel zu umgehen.

$337 \mathrm{Vgl}$. 8. Kapitel, in dem der Versuchsaufbau beschrieben wird.

338 Vgl. 2. Kapitel, Abschnitt I.2.1.

339 Die Möglichkeiten, die den MARGA-Teams zur Verfügung stehen, um Informationen über die Wettbewerber zu erhalten, werden im Abschnitt II.7.4 ausführlich dargestellt. 
Die Möglichkeit der indirekten Kommunikation endet erst mit der Hauptrunde des Planspiel-Wettbewerbs, nämlich nach fünf Perioden bei THE BOARD und nach sechs Perioden bei MARGA, was allen Teams von vorne herein bekannt ist. Danach gibt es nur den Erfolg, sich für das Viertelfinale zu qualifizieren und dort auf drei neue Konkurrenten zu stoßen, oder den Misserfolg auszuscheiden. Für die Wettbewerbssituation im Planspiel hat dies die Konsequenz, dass die Teams bei MARGA und bei THE BOARD ein Spielfeld haben, auf dem sie anonym und völlig frei von äußeren Zwängen des realen Lebens, allerdings unter Beachtung aller Spielregeln, agieren können. Das Ziel dabei ist es, in seiner Vierergruppe erstplatziert zu sein. Es ist demnach davon auszugehen, dass jedes Team eigene Stärken bestmöglich einsetzt und Schwächen von Wettbewerbern zum eigenen Vorteil konsequent ausnutzt.

Zur Erreichung des Ziels, einen höheren kumulierten Gewinn über alle Perioden der Hauptrunde zu erwirtschaften, als die drei Konkurrenten, stehen den Unternehmen mehrere strategische Möglichkeiten zur Verfügung. In Anlehnung an die vorherigen, theoretischen Ausführungen lassen sich auch bei MARGA drei generelle Wettbewerbsstrategien betrachten, deren Realisierung jeweils bestimmte Maßnahmenbündel erfordern: 340

1. Die Kostenführerschaft, die unter Ausnutzung des Erfahrungskurven-Effektes, der auch im Planspiel simuliert wird, und von Skaleneffekten mit einer hohen Produktionsmenge einhergeht. Begleitend kommen ein im Vergleich zu den Wettbewerbern expansives Investitionsverhalten und ein aggressives Marketing hinzu. Dieses kann bei MARGA durch niedrige Preise, hohe Forschungs- und Entwicklungs-Budgets, sowie durch hohe Ausgaben für Werbung und Vertrieb realisiert werden.

2. Die Differenzierung, die bei MARGA durch eine hohe Produktqualität oder durch ein hohes Werbe- oder Vertriebs-Budget zu realisieren ist. Das Preisniveau liegt im Vergleich zu den drei Wettbewerbem eher im mittleren bis oberen Bereich, wodurch die Marktanteile und damit auch die Produktionsmenge gewöhnlich niedriger sind, als bei einer Kostenführerstrategie, bei der eine Erhöhung der Marktanteile angestrebt wird. 
3. Die Konzentration auf Schwerpunkte kann bei MARGA segmentweise auf Produkt- oder auf Marktebene erfolgen. Dabei ziehen sich die Unternehmen aus einem oder mehreren der zwölf Marktsegmente zurück und konzentrieren sich auf die wirtschaftlich attraktivsten Segmente.

Während die Kostenführerschaft und die Differenzierung aufgrund der Rahmendaten der Hauptrunde von MARGA '99 und von THE BOARD '99 durchaus erfolgversprechende Strategien darstellen, ist die Konzentration auf Schwerpunkte aus mehreren Gründen als problematisch anzusehen. Diese strategische Ausrichtung ist bei MARGA nur vorteilhaft, wenn

- die Deckungsbeiträge in einzelnen Marktsegmenten deutlich negativ sind und damit jede nicht verkaufte Produkteinheit den Gewinn erhöht, oder

- limitierte Kapazitäten die Unternehmensleitung zu einer Konzentration auf attraktive Marktsegmente zwingen.

Hinzu kommt, dass der Marktanteil des Unternehmens, das aus einem Marktsegment austritt, auf die verbleibenden Unternehmen aufgeteilt wird und diese damit wirtschaftlich gestärkt werden, solange sie im betreffenden Marktsegment noch einen positiven Deckungsbeitrag erwirtschaften, was in der Regel der Fall ist. Da alle MARGA-Unternehmen mit der identischen Ausgangssituation starten und daher weder auf einem Markt, noch bei einem Produkt Vorteile gegenüber anderen haben, ist bspw. die in der Realität oft vorhandene lokale Marktstärke bei MARGA nicht vorhanden. Die geringe Zahl an Marktaustritten in der vorliegenden empirischen Untersuchung zeigt an, dass die Teams diese Problematik erkannt haben.

Es stellt sich im Folgenden die Frage nach der Dramaturgie des Planspiel-Wettbewerbs, die natürlich das Entscheidungsverhalten der Teams beeinflusst. ${ }^{341}$

Nach der Testrunde, in der alle Teams über ca. sechs Wochen Gelegenheit haben, sich mit dem Regelwerk der Simulation, sowie mit dem MARGA-Unternehmen und seinen Stärken und Schwächen vertraut zu machen, beginnt die Hauptrunde. In der Regel fällen die Teams in der 1. Periode der Hauptrunde nur selten extreme Entscheidungen, weil man sich damit u.U. auf eine Strategie

341 Die im Folgenden erörterten Zusammenhänge beruhen auf den mehrjährigen beruflichen Erfahrungen des Autors mit dem Planspiel MARGA. 
festlegen würde, ohne zu wissen, wie sich die Konkurrenten positionieren. Auf diese Weise hat man in der 2. Periode die Möglichkeit, eine vorsichtig eingeschlagene Strategie entweder mit größerem Nachdruck zu verfolgen oder sie noch ohne große Nachteile - neu auf das jeweilige Verhalten der Wettbewerber auszurichten. In der 3. Periode hat erfahrungsgemäß die überwiegende Mehrzahl der Unternehmen die eigene strategische Ausrichtung gefunden, die nun konsequent umgesetzt wird. Die operativen Maßnahmen zur Realisierung einer Strategie, wie bspw. eine aggressive Preispolitik, erreichen in der 3 . und 4. Periode ihre deutlichste Ausprägung. Bei MARGA zieht sich diese Phase der Strategieverfolgung auch in die 5. Periode hinein, während bei THE BOARD die 5. Periode bereits die letzte der Hauptrunde ist. $\mathrm{Zu}$ vergleichen ist diese mit der 6. Periode bei MARGA. Zum Ende des Planspiels verlassen einige Unternehmen die eingeschlagene langfristige Strategie, um kurzfristige Optimierungen vorzunehmen. So versuchen bspw. viele Unternehmen durch eine aggressive Preispolitik eventuelle Lagerbestände zu reduzieren. Das Modell bestraft - in Form von hohen Kosten - ökonomisch unsinnige Entscheidungen, wie bspw. die Verschrottung aller Maschinen oder die Entlassung aller Mitarbeiter, so dass sich die völlige Auflösung der bisherigen Strategie und deren Ersatz durch eine ausschließlich vom Ende des Planspiels bestimmten Handlungsweise in keinem Fall einstellt. Trotzdem ist die jeweils letzte Periode im Rahmen einer empirischen Untersuchung nur begrenzt aussagefähig und wird daher bei der späteren Interpretation des Datenmaterials ausgeklammert. ${ }^{342}$

Zusammenfassend kann die Wettbewerbsintensität, die zwischen den Planspiel-Unternehmen herrscht, damit als sehr hoch eingeschätzt werden.

\section{II.7.4 Konkurrenzanalyse bei MARGA}

Unter den im vorherigen Abschnitt erläuterten intensiven Wettbewerbsbedingungen kommt der Konkurrenzanalyse eine wesentliche Bedeutung zu. Je weitgehender es einem Unternehmen gelingt, sich durch eine detaillierte Ana-

342 Dieser Nachteil trifft auf alle Unternehmensplanspiele zu, bei denen die Teilnehmer vor dem Ende des Planspiels die Anzahl der insgesamt simulierten Perioden kennen. Aus organisatorischen Gründen lässt sich dies weder bei MARGA bzw. bei THE BOARD, noch bei den anderen zur Auswahl stehenden Simulationsmodellen vermeiden, so dass die letzte Periode bei der Interpretation des Datenmaterials keine Berücksichtigung finden sollte. 
lyse in die Situation seiner drei relevanten Konkurrenten ${ }^{343}$ hineinzuversetzen, desto eher ist es möglich, deren zukünftige strategische Ausrichtung zu antizipieren. Ist eine Strategie frühzeitig erkennbar, zieht diese - mit einer gewissen Wahrscheinlichkeit - ein bestimmtes Verhalten des Wettbewerbers nach sich. Unter diesen Annahmen können bereits im Vorfeld Gegenmaßnahmen ergriffen werden.

Den MARGA-Unternehmen stehen regulär zwei Quellen zur Verfügung, mit deren Hilfe sie Informationen über ihre Konkurrenten gewinnen können: der Marktforschungsbericht und der Jahresbericht der MARGA-Unternehmen.

Der Marktforschungsbericht muss von den Teams in jeder Planspielperiode und in einem von vier Detaillierungsgraden explizit bestellt und dementsprechend bezahlt werden. Wie im 2. Kapitel, Abschnitt I.2.2, allgemein erläutert, erhalten die Teams von der Marktforschung insbesondere Marktdaten, die die Basis der Marketing-Entscheidungen bilden. Darüber hinaus enthält der Marktforschungsbericht u.a. Informationen über die Umsätze, die Werbe-, F\&E- und Vertriebsbudgets, sowie über die Jahresüberschüsse aller Wettbewerber. Exemplarisch ist in Abbildung 23 der Marktforschungsbericht einer MARGA-Vierergruppe für das Produkt 1 auf dem Markt 1 dargestellt. ${ }^{344}$

Der Jahresbericht der MARGA-Unternehmen wird den Teams automatisch, kostenlos und nur einmalig nach der 2. Periode zur Verfügung gestellt. Der Bericht enthält die Bilanzen sowie die Gewinn- und Verlustrechnungen aller vier Wettbewerber, die, wie im Planspiel simuliert, nach der 2. Periode die Jahresabschlusszahlen veröffentlichen. In Abbildung 24 wird der Jahresbericht der MARGA-Unternehmen exemplarisch dargestellt.

Neben diesen beiden genannten Quellen, dem Marktforschungsbericht und dem Jahresbericht, gibt es für die Planspiel-Unternehmen keine weitere Möglichkeit, Konkurrenzdatenmaterial zu erhalten. Aus beiden Berichten zusammen

343 Der von HAX/MAJLUF stammende Begriff der "relevanten Konkurrenten" wird im 2. Kapitel, Abschnitt 1.2.1, erläutert. Vgl. HAX/MAJLUF, 1991, S. $338 \mathrm{f}$.

344 Im Planspiel enthält der Marktforschungsbericht darüber hinaus Informationen zu den Produkten 2 und 3, sowie zu den Märkten 2, 3 und 4, die nach dem gleichen Schema strukturiert sind. Der unten stehenden Legende kann der Leser die Bedeutung der verwendeten Abkürzungen entnehmen. 


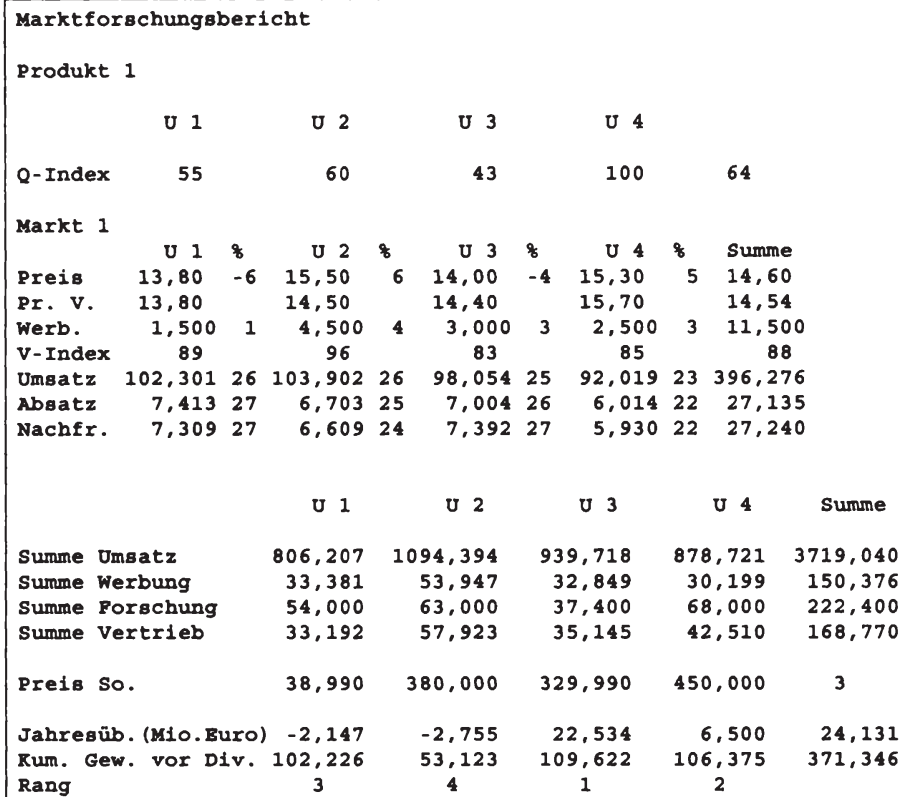

Legende:

U: $\quad$ Unternehmen 1 bis Unternehmen 4

Q-Index: Aufgerundeter Qualitäsindex je Produkt; Mafo 1: Rundung in 20er Stufen; Mafo 2: 10er Stufen; Mafo 3: 5er stufen; Mafo 4: ler stufen; Summe: Branchendurchschnittsqualität.

Pre1s: Preis Je Marktsegment; Markt 1: Euro; Markt 2-4: \$; 8 : Abweichung vom Branchendurchschnittspreis; Summe: Branchendurchschnittspreis.

Pr. V.: Prels der Vorperiode je Marktsegment; Markt 1: Buro; Markt 2-4: \$; Summe: Branchendurchschnittspreis der vorperiode

Werb.: Werbung Je Marktsegment; Markt 1: Mio. Euro; Markt 2-4: Mio. \$; 8: Antell an Omsatz der vorperiode.

V-Index: Aufgerundeter Vertriebsindex fe Markt; Summe: Branchendurchschnittsvertriebsindex; Mafo 1: Rundung in 20er Stufen; Mafo 2: 10er Stufen; Mafo 3: 5er Stufen; Mafo 4: 1er stufen.

Umsatz: Umsatz Je Marktsegment; Markt 1: Mio. Buro; Markt 2-4: Mio. \$; 8: Umsatzanteil je Unternehmen.

Absatz: Absatzmenge je Marktsegment in Mio. Stück; 8: Absatzanteil fe Unternehmen; Summe: Gesamtabsatzmenge.

Nachfrage:Nachfragemenge je Marktsegment in Mlo. St.; is: Nachfrageanteil je Unternehmen Summe: Gesamtnachfrage.

Preis So.: Nafo 3: Summe = Unternehmen, das den zuschlag erhalten hat; Mafo 4: Prelsangebote für die Sonderausschreibung.

Jahresüb.:Jahresüberschuss vor Dividende in Mio. Euro; wird in schritten von 3 kio. aufgerundet.

Kum. Gew.: Kumullerter Gewinn bis zur jetzigen Periode; wird in Schritten von 3 Mo. aufgerundet.

Abbildung 23: Der MARGA-Marktforschungsbericht 


\begin{tabular}{|c|c|c|c|c|}
\hline \multicolumn{5}{|l|}{ Jahresbericht der MARGA-Unternehmen } \\
\hline \multicolumn{5}{|l|}{ Bilanzen (Mio. Euro) } \\
\hline Aktiva & U 1 & U 2 & U 3 & U 4 \\
\hline & --- & -- & --- & -- \\
\hline Anlagevermögen & 384,150 & 544,750 & 488,350 & 513,350 \\
\hline Grundstück und Gebäude & 48,200 & 48,200 & 48,200 & 48,200 \\
\hline Technische Anlagen & 335,950 & 496,550 & 440,150 & 465,150 \\
\hline Umlaufvermögen & 763,305 & 1250,739 & 650,316 & 706,646 \\
\hline Vorräte & 349,116 & 531,501 & 238,413 & 114,418 \\
\hline Rohstoffe & 21,225 & 12,971 & 57,087 & 30,117 \\
\hline Fertigwaren & 327,891 & 518,531 & 181,327 & 84,301 \\
\hline Forderungen & 405,251 & 659,392 & 375,887 & 439,361 \\
\hline aus Warenlieferung Europa & 166,219 & 231,476 & 142,168 & 155,013 \\
\hline aus Warenlieferung Ausland & 236,884 & 425,160 & 233,719 & 284,347 \\
\hline Sonstige & 2,147 & 2,755 & 0,000 & 0,000 \\
\hline Wertpapiere & 0,000 & 0,000 & 36,015 & 0,000 \\
\hline Rasse+Guthaben & 8,939 & 59,845 & 0,000 & 152,868 \\
\hline Summe Aktiva & 1147,455 & 1795,489 & 1138,666 & 1219,996 \\
\hline \multicolumn{5}{|l|}{ Passiva } \\
\hline & $\ldots$ & -- & -- & $\ldots$ \\
\hline Eigenkapital & 710,477 & 680,772 & 724,786 & 716,425 \\
\hline Gezeichnetes Kapital & 400,000 & 400,000 & 400,000 & 400,000 \\
\hline Gewinnrücklagen & 312,624 & 283,527 & 302,253 & 309,925 \\
\hline Jahregüberschuss/Fehlbetrag & $-2,147$ & $-2,755$ & 22,534 & 6,500 \\
\hline Verbindlichkeiten & 436,979 & 1114,717 & 413,879 & 503,572 \\
\hline bei Rreditinstituten & 316,929 & 827,588 & 219,837 & 342,429 \\
\hline vor Abl. 1 J. fllg. & 6,214 & 16,873 & 8,807 & 6,714 \\
\hline Ausgleichskredit & 0,000 & 0,000 & 50,316 & 0,000 \\
\hline Restlfz. mehr als $5 \mathrm{~J}$. & 310,714 & 810,714 & 160,714 & 335,714 \\
\hline aus Lief. u. Leist. & 120,050 & 254,179 & 143,449 & 154,643 \\
\hline aus ausstehnden Wechseln & 0,000 & 32,950 & 28,060 & 0,000 \\
\hline aus steuern & 0,000 & 0,000 & 22,534 & 6,500 \\
\hline Summe Passiva & 1147,455 & 1795,489 & 1138,666 & 1219,997 \\
\hline \multicolumn{5}{|c|}{ Gewinn und Verlustrechnung (Mio.Buro) } \\
\hline Umsatzerlös & 802,176 & 1092,206 & 942,211 & 874,328 \\
\hline davon Umsatz Produkt 1 & 242,994 & 360,287 & 308,668 & 290,430 \\
\hline davon Umsatz Produkt 2 & 90,562 & 103,158 & 106,359 & 88,224 \\
\hline davon Umsatz Produkt 3 & 468,620 & 628,760 & 527,183 & 495,674 \\
\hline Herstellungskosten & 521,866 & 715,012 & 622,676 & 591,637 \\
\hline Bruttoergebnis vom Umsatz & 280,310 & 377,194 & 319,535 & 282,690 \\
\hline Vertriebskosten & 106,650 & 171,103 & 105,457 & 104,555 \\
\hline Allg. Verwaltungskosten & 52,089 & 54,093 & 46,946 & 24,781 \\
\hline Abschreibung Anlagevermögen & 27,600 & 35,000 & 29,400 & 24,400 \\
\hline Sonst. betriebl. Aufwendungen & 75,343 & 92,691 & 63,691 & 89,991 \\
\hline zinsen u. ähnl. Aufwendungen & 6,214 & 16,873 & 8,807 & 6,714 \\
\hline zingen für Finanzanlagen & 0,000 & 0,000 & 1,015 & 0,000 \\
\hline Liquidationserlöse & 0,280 & 0,000 & 0,000 & 0,000 \\
\hline Abschreibung auf Wertpapiere & 0,000 & 0,000 & 0,000 & 0,000 \\
\hline Operatives Brgebnis & $-4,294$ & $-5,511$ & 45,067 & 13,001 \\
\hline steuern & $-2,147$ & $-2,755$ & 22,534 & 6,500 \\
\hline Einbeh. Gewinn/Fehlbetrag & $-2,147$ & $-2,755$ & 13,520 & 3,900 \\
\hline
\end{tabular}

Abbildung 24: Der MARGA-Jahresbericht 
können jedoch eine Vielzahl direkt und indirekt verwertbarer Wettbewerbsinformationen abgeleitet werden.345 So sind die Angaben über die in den einzelnen Marktsegmenten von den Wettbewerbern eingesetzten Marketinginstrumente Preis, Werbung, Vertrieb und Produktqualität sowie über die jeweiligen Absatz- und Umsatzanteile sicherlich direkt im Rahmen einer Konkurrenzanalyse verwendbar. Ergänzend lassen sich indes einige der im 3. Kapitel genannten Instrumente einsetzen, wie in den weiteren Ausführungen dargestellt wird.

- Die Bilanzanalyse kann, wie zuvor erläutert, von den Teams nach der 2. Periode auf der Basis des Jahresberichts durchgeführt werden. ${ }^{346}$ Dieser verzichtet jedoch aus organisatorischen Gründen auf den Anhang und den Lagebericht. Gemäß der Ausführungen im 3. Kapitel, Abschnitt 1.3.3, können aus dem Jahresabschluss diverse finanz- und erfolgswirtschaftliche Informationen gewonnen werden. Darüber hinaus lassen sich strategisch-taktische Rückschlüsse auf Konkurrenzunternehmen ziehen. So deutet bspw. ein relativ hohes Anlagevermögen darauf hin, dass das betreffende Unternehmen maschinelle Kapazitäten aufgebaut hat. Bei einer entsprechenden Auslastung ist demnach davon auszugehen, dass aufgrund des gestiegenen Angebots ein Verdrängungswettbewerb erfolgt, der häufig durch aggressive Marketing-Politik gekennzeichnet ist.

- Die Erfahrungskurve ${ }^{347}$ ist im Planspiel integriert und wird von den Teams nach der 2. Periode quantitativ exakt für jedes der drei Produkte ermittelt. Mit Hilfe der im Lieferumfang von MARGA enthaltenen MARGA-Support Software haben die Teams die Möglichkeit, die kostenrelevanten Auswirkungen des Erfahrungskurveneffektes für das eigene Unternehmen zu prognostizieren. Damit steht auch Teilnehmern, die nicht kaufmännisch vorgebildet sind, ein Hilfsmittel zur Verfügung, das ihnen die praktische Relevanz dieses Effektes vermittelt. Abbildung 25 stellt den entsprechenden Bereich der Software dar.

345 Im 4. Kapitel, Abschnitt 1.4.2, werden der Daten- und Informationsfluss sowie der generelle Einsatz von Instrumenten in der Konkurrenzanalyse dargestellt, worauf hier Bezug genommen wird.

346 Damit ist gewährleistet, dass die Teams die in der Bilanzanalyse gewonnenen Erkenntnisse in Entscheidungen umsetzen und sich diese in den folgenden drei bzw. vier Perioden auswirken können. Vgl. 7. Kapitel, Abschnitt II.7.2.

347 Vgl. 3. Kapitel, Abschnitt I.3.5. 


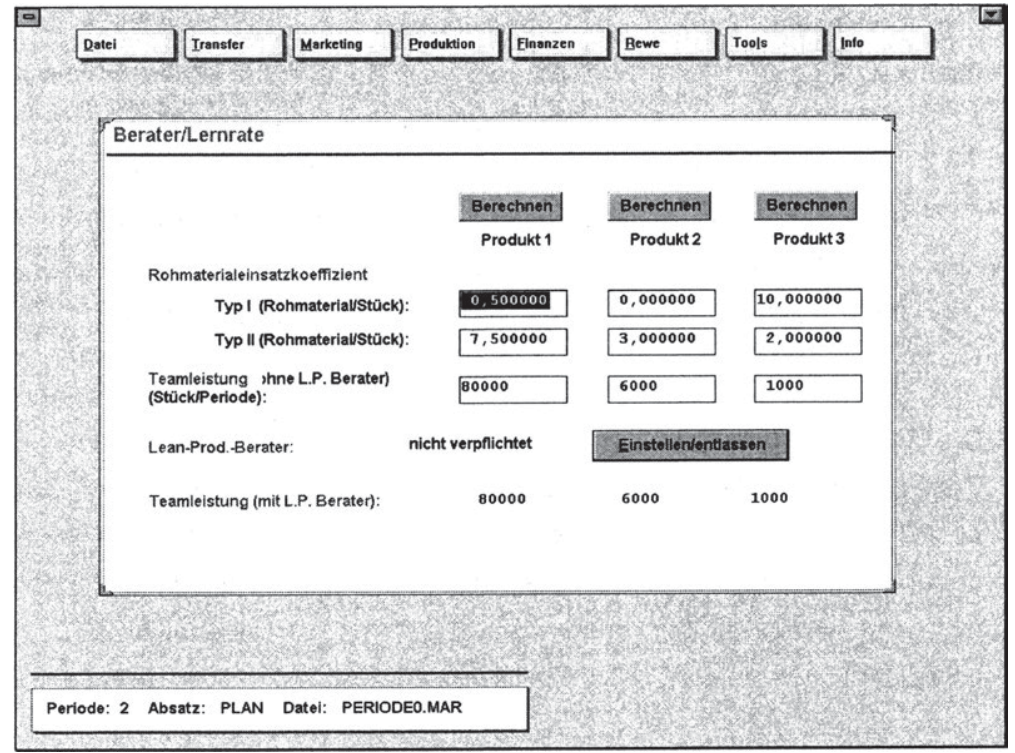

Abbildung 25: Die Erfahrungskurve in der MARGA-Support Software

Hat ein Unternehmen die eigenen Erfahrungskurveneffekte bestimmt, kennt es damit auch die der Wettbewerber, denn diese sind, ähnlich wie in der Realität, für alle Hersteller eines Produktes gleich. Diese Vergleichbarkeit gilt nur solange kein außergewöhnlicher technischer Fortschritt, deutliche Produktinnovationen oder strukturelle Veränderungen der Wertschöpfungskette erfolgen, was im Planspiel modellbedingt ausgeschlossen ist. Trotzdem können die Teams aus den im Marktforschungsbericht ausgewiesenen Absatzzahlen nur in sehr begrenztem Umfang Rückschlüsse auf die Herstellkosten der Konkurrenten ziehen. Die Herstellkosten setzen sich bei MARGA aus den Material- und Personalkosten zusammen, die auf vielen unterschiedlichen Kostenkombinationen basieren, welche wiederum aus der individuellen Einkaufs- und Personalpolitik der Unternehmen resultieren. ${ }^{348}$ Somit lässt sich aus den Absatzzahlen der Wettbewerber unter Verwendung des Erfahrungskurveneffektes nicht zuverlässig herausfinden, ob ein Unternehmen vergleichsweise hohe oder niedrige Herstellkosten aufweist. Dies 
kann nur unter der unwahrscheinlichen Annahme erfolgen, dass die Konkurrenten die gleiche Einkaufs- und Personalpolitik betreiben.

- Die Portfolio-Analyse 349 erfolgt im Planspiel MARGA mit Hilfe des von der Boston Consulting Group entwickelten Ansatzes, dem BCG-Portfolio. Wie in Abbildung 26 zu sehen ist, bildet die Abszisse dabei den relativen Marktanteil ab, während auf der Ordinate das Marktwachstum abgetragen wird. Das BCG-Portfolio steht jedem Team in der Planungssoftware, die im Lieferumfang des Planspiels enthalten ist, zur Verfügung. Damit sind alle Teams in der Lage, für jeden Wettbewerber eine umfassende Portfolio-Analyse durchzuführen, bei der alle Produkte und Märkte über den Zeitverlauf oder als Momentaufnahme betrachtet werden können.

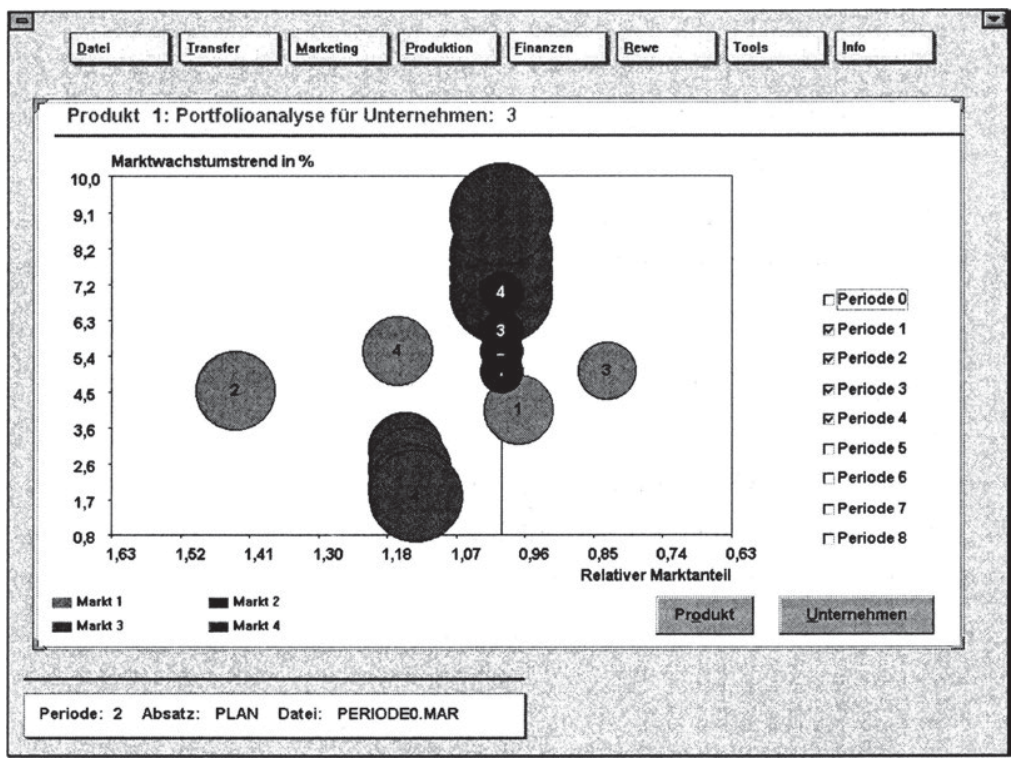

Abbildung 26: Das BCG-Portfolio in der MARGA-Support Software

- Die Wertkettenanalyse ${ }^{350}$ ist, im Gegensatz zu ihrer großen Bedeutung in der Praxis, bei MARGA nur begrenzt anwendbar, da alle Planspiel-Unternehmen mit der gleichen Ausgangssituation starten und während des Spiel-

349 Vgl. 3. Kapitel, Abschnitt 1.3.7.

$350 \mathrm{Vgl}$. 3. Kapitel, Abschnitt I.3.6. 
verlaufs nur geringe strukturelle Änderungen der Wertschöpfungskette vorgenommen werden können. ${ }^{351}$

- Bei der Stärken-/Schwächen-Analyse ${ }^{352}$ werden zunächst Unternehmensmerkmale gesucht, die für den zukünftigen wirtschaftlichen Erfolg bedeutsam sind. Diese Merkmale werden mit den relevanten Wettbewerbern verglichen, so dass sich ein Stärken-/Schwächen-Profil ergibt. Dieses Vorgehen ist auf die Planspielsituation übertragbar, wenn die Teams aus den beiden Quellen die entsprechenden Konkurrenzinformationen als Vergleichsgrundlage ableiten können. Wird bspw. der Produktqualität eine besondere Bedeutung beigemessen, kann diese auf der Grundlage des Marktforschungsberichtes für alle vier Unternehmen bestimmt und verglichen werden.

- Der Einsatz von Checklisten im Rahmen der Konkurrenzanalyse ${ }^{353}$ ist auch im Planspiel möglich und bietet den Teams den Vorteil, institutionalisiert, d.h. in regelmäßigen Abständen bestimmte Unternehmensmerkmale der Wettbewerber, die für wichtig gehalten werden, zu erfassen. Die Inhalte der verwendeten Checkliste sind den individuellen Informationsbedürfnissen der jeweiligen Entscheidungsträger anzupassen.

- In das System zur Konkurrenzanalyse von PORTER 354 fließen direkte aber auch indirekt aus den genannten Instrumenten abgeleitete Informationen, so dass damit in vielen Fällen ein Reaktionsprofil einzelner Wettbewerber abgeleitet werden kann. So sei auf das zuvor dargestellte Beispiel des erhöhten Anlagevermögens verwiesen, das durch eine Bilanzanalyse festzustellen ist. Über direkte Marketinginformationen, wie z.B. steigende Absatzzahlen oder eine aggressivere Marketingpolitik lässt sich die vermutete Verdrängungsstrategie verifizieren und auf einzelne Produkte einschränken. Das Kostensenkungspotential ist durch den Erfahrungskurveneffekt quantifizierbar, wodurch zusätzlich das Preissenkungspotential des Konkurrenten

351 Die Unternehmen haben bspw. die Möglichkeit, statt der Eigenfertigung von Produkten Handelsware zu beziehen. Dies ist allerdings nur in sehr begrenztem Umfang möglich, bspw. um kurzfristige Produktionsengpässe auszugleichen, nicht jedoch um eine langfristige Outsourcing-Strategie zu wählen.

352 Vgl. 3. Kapitel, Abschnitt I.3.1.

353 Vgl. 3. Kapitel, Abschnitt I.3.2.

354 Vgl. 3. Kapitel, Abschnitt I.3.4. 
im Falle eines Preiskriegs abgeschätzt werden kann. Die Portfolio-Analyse stellt grafisch auf der Grundlage von Zahlen des Marktforschungsberichts das Marktwachstum und den relativen Marktanteil dar und verdeutlicht die strategische Situation des betreffenden Unternehmens. Das Reaktionsprofil einzelner Wettbewerber kann auf diese Weise hergeleitet werden.

Allen Planspiel-Unternehmen stehen, wie gezeigt, zwei Datenquellen als Grundlage ihrer Wettbewerbsbetrachtung zur Verfügung, aus denen sich direkt und indirekt verwertbare Informationen ableiten lassen. Weiterhin können von den Teams verschiedene Instrumente eingesetzt werden, mit denen diese Basisinformationen aufbereitet werden können. Es ergibt sich demnach ein konkretes Bild einzelner Wettbewerber, das aus unterschiedlichen Blickwinkeln betrachtet und bis hin zu einem wahrscheinlichen Reaktionsprofil verfeinert werden kann. Auf diese Weise haben die Teams die Möglichkeit, durch eine entsprechend detaillierte Konkurrenzanalyse der im vorherigen Abschnitt festgestellten intensiven Wettbewerbssituation im Planspiel Rechnung zu tragen. 


\section{Kapitel Versuchsaufbau}

Der Versuchsaufbau der vorliegenden empirischen Studie hat sich einerseits an den zu untersuchenden inhaltlichen Zusammenhängen und andererseits an dem zur Verfügung stehenden Instrumentarium und seinen Möglichkeiten zu orientieren. Der Versuchsaufbau ist die Umsetzung der zu analysierenden Problematik, ausgedrückt in Hypothesen, in ein Experiment, das diese Hypothesen widerlegt oder bestätigt. $\mathrm{lhm}$ kommt damit eine sehr wichtige Aufgabe in der vorliegenden Arbeit zu, weil er das Bindeglied zwischen Inhalt und Instrument darstellt.

Während die im 5. Kapitel erörterte Kostenbetrachtung in der Konkurrenzanalyse den inhaltlichen Rahmen vorgibt, wird im 7. Kapitel das in dieser Studie zum Einsatz kommende Unternehmensplanspiel MARGA erläutert. In den nun folgenden Ausführungen werden beide Aspekte zu einem Versuchsaufbau zusammengeführt.

Der Ausgangspunkt ist die im 5. Kapitel, Abschnitt 1.5.2, hergeleitete Grundhypothese, die Folgendes besagt: Die Kostenbetrachtung von Wettbewerbern im Rahmen der Konkurrenzanalyse lässt die eigenen Stärken und Schwächen auf der Kostenebene erkennen. Die Kenntnis dieser Stärken und Schwächen führt zu einer Zunahme an Planungs- und Entscheidungssicherheit, die die strategischen Entscheidungen insbesondere von Kostenführern beeinflussen wird. Dieser Zusammenhang wird sich positiv auf den Unternehmenserfolg auswirken.

Unternehmen, die über die Kosteninformationen von Wettbewerbern verfügen, sollten damit andere strategische Entscheidungen fällen und damit letztlich erfolgreicher sein, als Unternehmen, die über diese Informationen nicht verfügen.

\section{II.8.1 Anpassung des Instrumentariums und der Versuchsanordnung an die inhaltlichen Erfordernisse der Untersuchung}

Im Folgenden ist das Instrument, dessen sich im Rahmen dieser Studie bedient wird, nämlich das Unternehmensplanspiel MARGA, daraufhin zu analysieren, inwiefern Wirkungszusammenhänge unzweifelhaft bestimmten Ursachen zuzu- 
ordnen sind und vorhandene Störgrößen ausgeschaltet werden können. Entsprechend sind das Instrument und die Versuchsanordnung zu gestalten.

Die empirische Untersuchung wird getrennt für die Planspiele MARGA und THE BOARD durchgeführt, weil

1. die Anzahl der simulierten Perioden unterschiedlich ist, d.h. bei MARGA sechs und bei THE BOARD fünf Perioden. Da in der jeweils letzten Spielperiode ein kurzfristiges und vom bevorstehenden Spielende geprägtes Entscheidungsverhalten der Teams zu enwarten ist, kann ab der 5. Periode keine unzweifelhafte Vergleichbarkeit beider Planspiele gewährleistet werden. 355 Und weil

2. die Teilnehmerstruktur, sowie deren Ausbildungsstand bzw. deren Erfahrungshintergrund unterschiedlich sind. Während MARGA in der betrieblichen Weiterbildung für Führungskräfte eingesetzt wird, nehmen an THE BOARD ausschließlich Studenten teil.

Alle teilnehmenden 284 MARGA-Teams und 472 THE BOARD-Teams werden nach dem Zufallsprinzip in Gruppen zu je vier Unternehmen aufgeteilt, wobei nur diese vier Konkurrenten im direkten Wettbewerb zueinander stehen. Sie haben keine direkten Kontaktmöglichkeit. Demnach gibt es 71 MARGA- und 118 THE BOARD-Gruppen mit jeweils vier Teams in jeder Gruppe.

Die 71 MARGA- und 118 THE BOARD-Gruppen werden einer Untersuchungsoder einer Kontrollmenge zugeteilt, so dass sich folgende Aufteilung ergibt:

\begin{tabular}{|l|c|c|}
\cline { 2 - 3 } \multicolumn{1}{c|}{} & Untersuchungsmenge & Kontrollmenge \\
\hline MARGA & Gruppe 1-36 & Gruppe 37-71 \\
\hline THE BOARD & Gruppe 1-60 & Gruppe 61-118 \\
\hline
\end{tabular}

Tabelle 4: Die Aufteilung der MARGA- und der BOARD-Teams

Alle Teams, die über ihre Gruppenzuteilung der Untersuchungsmenge zugeordnet werden, erhalten bestimmte Wettbewerbsinformationen. Die Teams, die der Kontrollmenge zugeteilt werden, erhalten diese Informationen nicht.

355 Das strategische Entscheidungsverhalten wird im 7. Kapitel, Abschnitt II.7.3, dargestellt. 
Innerhalb einer Vierergruppe und innerhalb der jeweiligen Untersuchungs- bzw. Kontrollmenge wird somit eine gleiche Informationsbasis geschaffen. Dabei muss aus inhaltlichen und didaktischen Gründen eine Ausnahme gemacht werden: Jedes Team sollte frei entscheiden können, welchen Marktforschungsbericht es in den jeweiligen Perioden ordert. ${ }^{356}$ Durch eine entsprechende Preisgestaltung seitens der Planspielleitung konnte die große Mehrzahl aller Teams in ihrer Entscheidung für den detaillierten Marktforschungsbericht "vier" beeinflusst werden. Wie sich während der Untersuchung jedoch herausstellte, gab es auch Teams, die in einer der sechs bzw. fünf Perioden nicht den Marktforschungsbericht "vier" geordert haben, sondern einen weniger detaillierten. Diese Teams wurden aufgrund des in diesem Fall vorhandenen unterschiedlichen Informationsstandes von der Untersuchung ausgeschlossen, obwohl der Informationsnachteil als relativ gering einzustufen ist.

Darüber hinaus ist ein weiteres Ausschlusskriterium zu beachten: Es besteht die Möglichkeit, dass ein Teams aus einem der vier Marktsegmente aussteigt, auf dem es das Produkt als Kostenführer anbietet. Auch in diesem Fall wird das betreffende Team für das vorliegende Experiment nicht zugelassen. ${ }^{357}$

Die empirische Untersuchung konzentriert sich auf die Betrachtung strategischer Entscheidungen von Kostenführern. ${ }^{358}$ Jedes MARGA-Unternehmen bietet insgesamt drei Produkte an, so dass es in jeder Vierergruppe drei Kostenführer gibt. Ausschließlich deren Entscheidungen werden für jedes der drei Produkte in einzelnen Datensätzen erfaßt und in der empirischen Untersuchung ausgewertet. Damit enthält jeder Datensatz nur Entscheidungsdaten eines Unternehmens, das sich in einer kostenführenden Position befindet.

Unter einem Kostenführer wird im Rahmen dieser Untersuchung das Unternehmen verstanden, das zu einem bestimmten Zeitpunkt, nämlich am Ende der 2. Periode, die geringsten variablen Produktionskosten je Stück eines Produktes 1, 2 oder 3 innerhalb seiner Vierergruppe aufweist. Demnach können in

356 Im 7. Kapitel, Abschnitt II.7.4, wird der Marktforschungsbericht ausführlich dargestellt.

357 Ein genauer Überblick über die Anzahl der letztlich gewerteten Teams erfolgt bei der Hypothesenformulierung in den nachfolgenden Abschnitten II.8.2.1 bis II.8.2.6.

358 Für Unternehmen, die eine Strategie der Kostenführung verfolgen, ist die Kenntnis von Stärken und Schwächen auf der Kostenebene von größter Wichtigkeit. Vgl. dazu 1. und 5. Kapitel, Abschnitt 1.1.3.3 und I.5.2. 
einer Vierergruppe drei verschiedene Unternehmen die Kostenführerschaft für je eines der drei Produkte inne haben.

Die variablen Kosten sind bei MARGA aus didaktischen Gründen ausschließlich durch die Material- und die Personalkosten bestimmt. Die Materialkosten leiten sich aus dem Einkauf zweier Rohstofftypen I und II ab, bei denen Mengenrabatte, Lager- und Finanzierungskosten zu beachten sind. Die Personalkosten sind abhängig von der Anzahl, der zur Produktion notwendigen Arbeitnehmer, der Höhe der Löhne und vom Sozialkostensatz. Die Produktionsmengen der Produkte 1, 2 und 3 und die davon abgeleitete Anzahl und Aufteilung der notwendigen Fach- und Hilfsarbeiter werden vom Team entsprechend der gewählten Strategie gesteuert. Die Löhne sind für alle Teams einheitlich und nicht veränderbar. Über den Sozialkostensatz können die Teilnehmer die Höhe der Sozialleistungen wählen, wobei sie damit den Krankenstand, die Fluktuation und damit wiederum die Personalkosten beeinflussen. ${ }^{359}$

Steht die Anzahl der benötigten Fach- und Hilfsarbeiter fest, können die MARGA-Unternehmen diesen Bedarf durch Einstellungen, Leiharbeiter, Überstunden oder Umschulungsmaßnahmen decken, wobei jede Maßnahme unterschiedliche Kosten verursacht. Die gesamten Material- und Personalkosten werden darüber hinaus durch den Erfahrungskurveneffekt 360 beeinflusst, der zu einer generellen Senkung dieser Kosten im Planspielverlauf in Abhängigkeit von der kumulierten Produktionsmenge der Produkte 1, 2 und 3 beiträgt. Ein Unternehmen hat keine Möglichkeit, bspw. durch Automatisierung, variable Kosten durch steigende Fixkosten zu substituieren und sich so zum Kostenführer im Sinne dieser Arbeit zu entwickeln. ${ }^{361}$

Es besteht die Möglichkeit, dass die Kostenführerschaft zwischen der 3. und 6 . Periode wechselt. In diesem Fall wäre damit zu rechnen, dass der ehemalige Kostenführer seine bis zu diesem Zeitpunkt durchgeführte Kostenführerstrate-

359 Bei einer Senkung der Sozialleistungen steigen der Krankenstand und die Fluktuation. Bei der Erhöhung des Sozialkostensatzes gilt der Umkehrschluss.

360 Die Erfahrungskurve wird im 3. Kapitel, Abschnitt 1.3.5, grundlegend dargestellt. Die Bedeutung der Erfahrungskurve als Instrument der Konkurrenzanalyse im Rahmen des Planspiels MARGA wird im 7. Kapitel, Abschnitt II.7.4, erläutert.

361 Diese relative "Stabilität" der Planspielsituation wird für die durchzuführende Untersuchung als vorteilhaft bewertet. Da die grundlegende Struktur des simulierten Unternehmens für alle Teams gleich bleibt und nur die strategische Ausrichtung variiert werden kann, sind die einzelnen Unternehmen und ihre gewählten Strategien eindeutiger zu vergleichen. 
gie aufgibt, obwohl der Grund dafür nicht in Verbindung steht mit den Zusammenhängen, die in der vorliegenden Studie untersucht werden sollen. Um diese Störgröße auszuschließen, erhalten die Teams der Untersuchungsmenge die Kosteninformation nur einmalig nach der 2. Periode. Damit wird erreicht, dass der nach der 2. Periode feststehende Kostenführer einen eventuellen Positionswechsel in der Kostenführung nicht erfährt und er daher unbeeinflusst eine gewählte Strategie fortsetzen kann, selbst wenn die Kostenführung wechseln sollte.

Alle Teams der Untersuchungsmenge erhalten am Ende der 2. Periode eine Information darüber, welches der vier Unternehmen einer Gruppe bei den jeweiligen Produkten 1, 2 und 3 die Kostenführung inne hat. Durch die Beschränkung rein auf die Information der Kostenführerschaft wird die Studie auf eine breitere Basis gestellt, weil davon auszugehen ist, dass diese Information im Rahmen der Konkurrenzanalyse in der Realität einfacher zu beschaffen ist, als detaillierte quantitative Kosteninformationen von Wettbewerbern. ${ }^{362}$ Insofern wird in dieser Studie unterstellt, dass ein Unternehmen, das über detailliertere Kosteninformationen von Wettbewerbern verfügt, ebenso den Kostenführer der Branche kennt. Der Umkehrschluss, dass nämlich ein Unternehmen, dem der

TICKER!! TICKER!! TICKER !!

Durch eine aufmerksame Konkurrenzanalyse ist es Ihnen gelungen folgende Informationen herauszufinden:

Kostenführer für das Produkt 1 ist:Unternehmen $X$ Kostenführer für das Produkt 2 ist:Unternehmen $Y$ Kostenführer für das Produkt 3 ist:Unternehmen $Z$

Kostenführer in diesem Sinne ist das Unternehmen, das nach der 2. Periode die niedrigsten variablen Produktionskosten je Stück gemäß Kostenbericht S. 8 aufweist. Diese Information erhalten Sie nur einmal!

Abbildung 27: Die Kosteninformation für Teams der Untersuchungsmenge

362 Die Beschaffung von Kosteninformationen in der Konkurrenzanalyse steht im Mittelpunkt des 4. Kapitels, Abschnitt I.4.2. 
Kostenführer der Branche bekannt ist, auch Detaillinformationen über Kosten von Konkurrenten besitzt, wird hier nicht zugelassen. Insofern stellt diese Verallgemeinerung eine Erweiterung der Studie dar.

Abbildung 27 zeigt die Information, die die Teams der Untersuchungsmenge am Ende der 2. Periode erhalten.Dabei werden $X, Y, Z$ durch die jeweiligen Unternehmensnummern 1, 2, 3 oder 4 ersetzt. Die Teams der Kontrollmenge erhalten diese Information nicht und haben auch keine Möglichkeit, diese zu beschaffen oder eigenständig aus verschiedenen Informationen schlusszufolgern.

Um sicherzustellen, dass die Kosteninformationen von jedem Wettbewerber trotz des mit dreizehn Seiten großen Informationsangebots ${ }^{363}$ am Ende der 2. Periode wahrgenommen werden, wird die auffällige Form der Tickermeldung gewählt. Die Gefahr eines zu hohen Aufmerksamkeitsgrades, der das Entscheidungsverhalten der Teams beeinflusst bzw. sogar verfälscht, wird als vernachlässigbar gering eingestuft, zumal es durchaus positiv zu bewerten ist, wenn Teams erst durch die Tickermeldung auf bestimmte Zusammenhänge gestoßen werden. Im Übrigen stellt die grundsätzliche Wahmehmung der zur Verfügung gestellten Kosteninformationen die Basis des gesamten Experiments dar und muss daher sichergestellt sein.

Durch diese Information erfahren die Kostenführer von ihrer relativen Wettbewerbsstärke auf der Kostenebene. Die Kenntnis inrer günstigen Kostenposition innerhalb ihrer Branche, also der Vierergruppe, können die Kostenführer für ihre weiteren Entscheidungen nutzen. Es liegt in dieser Situation nahe, sich als Kostenführer, der nun erstmals die sichere Erkenntnis seiner Stärke hat, für eine Kostenführerstrategie zu entscheiden. Durch die bereits dargestellte Strategie der Kostenführung ${ }^{364}$ nutzen die Kostenführer einerseits ihre Stärke gegenüber den Konkurrenten aus, indem sie bspw. eine aggressivere Preispolitik wählen können, als ihre Wettbewerber, die zu höheren Kosten produzieren. Andererseits können sie gleichzeitig durch ein aggressives Marketing ein starkes Mengenwachstum erreichen, was wiederum Kostensenkungspotentiale und damit eine weitere Stärkung der Kostenposition ermöglicht.

363 Alle Teams erhalten nach jeder Simulation die Ergebnisse der letzten Periode.

364 Vgl. 1. Kapitel, Abschnitt I.1.3.3, 5. Kapitel, Abschnitt I.5.2 und 7. Kapitel, Abschnitt II.7.3. 
Der Zeitpunkt der Information nach der 2. Periode leitet sich aus folgenden Überlegungen $\mathrm{ab}$ : Da alle teilnehmenden Teams mit der identischen Ausgangssituation starten, gibt es zu Beginn des Planspielwettbewerbs keine Kostenführer. Erst nach zwei Spielperioden - eine Periode wäre zu wenig ${ }^{365}$ haben sich aufgrund vieler vorheriger Entscheidungen individuelle variable Kostenstrukturen herausgebildet, auf deren Basis eine Kostenführerschaft abgeleitet und begründet werden kann. Hinzu kommt, dass die Unternehmen nach der 2. Periode erfahrungsgemäß strategisch noch nicht endgültig festgelegt sind. ${ }^{366}$ Eine Anpassung der generellen strategischen Ausrichtung ist noch ohne große Nachteile möglich. Nach zwei Perioden sind bei MARGA noch vier und bei THE BOARD noch drei Perioden zu simulieren, so dass sich strategische Entscheidungen noch vor dem Ende des Wettbewerbs auswirken können.

Die gesamte Untersuchung ist produktweise aufgebaut. Betrachtet werden strategische Entscheidungen und der wirtschaftliche Erfolg der Kostenführer der Produkte 1, 2 und 3 einer Vierergruppe. Jedes Produkt wird auf vier Märkten, Europa, Russland, den USA und Japan angeboten. Die Entscheidungen eines Kostenführers bezüglich der Preispolitik und der Werbung kann marktweise, d.h. je Produkt vier Mal analysiert werden. Andere Entscheidungen der jeweiligen Kostenführer, wie das Investitionsverhalten, das Bestimmen der Produktionsmengen und die Forschungs- und Entwicklungsstrategie können nur produktweise, d.h. einmal je Produkt, entschieden und untersucht werden.

Zwischen den drei Produkten und zwischen den vier Märkten bestehen keine Beziehungen oder Abhängigkeiten. Die Produktion der drei Produkte erfolgt auf Maschinen, wobei jedes der drei Produkte nur auf einem von drei Maschinentypen hergestellt wird, wie Tabelle 5 zu entnehmen ist.

\begin{tabular}{|c|c|c|c|}
\cline { 2 - 4 } \multicolumn{1}{c|}{} & Maschinentyp A & Maschinentyp B & Maschinentyp C \\
\hline Produkt 1 & $\mathrm{X}$ & & \\
\hline Produkt 2 & & $\mathrm{X}$ & \\
\hline Produkt 3 & & & $\mathrm{X}$ \\
\hline
\end{tabular}

Tabelle 5: Die Maschinenbelegung der MARGA-Produkte

365 Diese Einschätzung beruht auf der beruflichen Erfahrung des Autors.

366 Siehe dazu die Ausführungen zum Wettbewerbsumfeld und zum strategischen Verhalten der Planspiel-Unternehmen im vorherigen 7. Kapitel, Abschnitt II.7.3.

Christoph Heinen - 978-3-631-75315-6 
Die Maschinentypen sind unabhängig voneinander und einzeln zu beschaffen. Jedes Produkt kann auf jedem Markt separat angeboten werden. Dafür stehen allen Teams die gleichen Instrumente zur Verfügung. Auf den zwölf Marktsegmenten gelten für alle Teams gleiche volkswirtschaftliche Rahmendaten.

Der wirtschaftliche Erfolg entspricht bei MARGA den kumulierten Jahresüberschüssen bzw. -fehlbeträgen aller simulierten Perioden. Dabei kommt es nicht auf die absolute Höhe des Ergebnisses an, sondern nur darauf, einen höheren Jahresüberschuss bzw. einen niedrigeren Jahresfehlbetrag kumuliert über alle Perioden erwirtschaftet zu haben, als die drei Wettbewerber. Auf der Basis der kumulierten Jahresüberschüsse bzw. -fehlbeträge werden die Platzierungen 1 bis 4 vergeben. Damit wird einerseits erreicht, dass die Platzierung der Teams auf der Grundlage eines finanziellen Ergebnisses basiert, das sich aus vielen Einzelentscheidungen in den Bereichen Marketing. Produktion und Finanzen über alle Perioden ergibt. Andererseits bleibt die Platzierung damit unabhängig vom Preisniveau, das sich in jeder Vierergruppe unterschiedlich entwickelt und finanzielle Ergebnis stark positiv oder negativ beeinflusst. So kann sich ein einzelnes Team einem sinkenden Preisniveau, bzw. einem Preiskampf, in seiner Vierergruppe mit den daraus folgenden negativen Auswirkungen auf das absolute finanzielle Ergebnis nicht vollständig entziehen. Dieses bei MARGA seit vielen Jahren verwendete Verfahren ermöglicht es, den wirtschaftlichen Erfolg eines Planspiel-Unternehmens vergleichbar zu machen mit Unternehmen, die einer anderen Vierergruppe angehören, in der sich die Märkte im Spielverlauf völlig unterschiedlich entwickelt haben und in der andere Preis- und Gewinnniveaus vorherrschen.

Für die vorliegende empirische Untersuchung soll wegen der oben erläuterten Gründe ebenfalls die Platzierung als Indikator für den wirtschaftlichen Erfolg der Teams verwendet werden. Dabei ist zu beachten, dass das Gesamtergebnis auf Aufwendungen und Erträgen basiert, die im regulären MARGA-Rechnungswesen großteils nur allen drei Produkten gemeinsam zugeordnet werden können, wie bspw. die Overhead-Kosten. Da aber die vorliegende Untersuchung produktweise durchgeführt wird, muss auch der Erfolg produktweise, d.h. verursachungsgemäß ermittelt werden. Dabei dürfen nur Aufwendungen und Erträge des Kostenführers verrechnet werden, die eindeutig dem Produkt zuzuweisen sind, bei dem die Kostenführung besteht. Ausschließlich zu diesem Zweck wird 
eine produktspezifische Deckungsbeitragsrechnung entwickelt, mit deren Hilfe eine verursachungsgerechte Kosten- und Erlöszuweisung erfolgen kann:

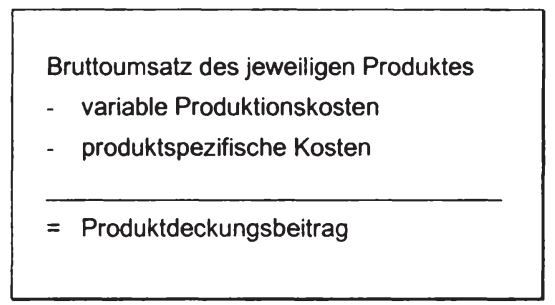

Abbildung 28: Die produktspezifische Deckungsbeitragsrechnung

Der Bruttoumsatz wird für das jeweilige Produkt, bei dem die Kostenführung besteht, über alle Märkte zum jeweils aktuellen Wechselkurs Euro/US-\$ ohne Abzug von Skonto und ohne Berücksichtigung von Sonderausschreibungen ${ }^{367}$ berechnet. Die variablen Produktionskosten ergeben sich aus dem, für die Produktion des jeweiligen Produktes notwendigen Material- und Personalbedarf. Die Materialkosten werden dabei nicht auf Basis einer sonst angebotenen Rabattstaffel für Rohmaterial, sondern zu einem einheitlichen Preis errechnet. Die Personalkosten ergeben sich aus dem Bedarf an Fach- und Hilfsarbeitern multipliziert mit dem für alle Teams geltenden Grundlohn. Sozialkostensätze, Leiharbeiter- und Überstundenzuschläge, Kosten für Einstellungen, Entlassungen, Urlaub oder Umschulungen werden für diesen Versuch ausgeklammert. So kann eine einheitliche Berechnungs- und Bewertungsbasis gewährleistet werden. Die produktspezifischen Kosten ergeben sich aus den produktbezogenen Aufwendungen für Forschung und Entwicklung, sowie für Werbung. Die Gemeinkosten höherer Ebenen werden nicht einbezogen, da keine Aussagen bezüglich der in der vorliegenden Studie zu untersuchenden Zusammenhänge daraus abgeleitet werden können und sie produktweise nicht eindeutig verursachungsgerecht zurechenbar sind.

367 Sonderausschreibungen sind in der MARGA-Terminologie Aufträge, die die Unternehmen bei MARGA zusätzlich zum Absatz der Produkte auf den vier Märkten annehmen können. Diese Aufträge werden öffentlich ausgeschrieben, wobei nur einer der vier Wettbewerber den Zuschlag erhält, nämlich der, der zum günstigsten Preis anbietet. Vgl. UNIVERSITÄTSSEMINAR DER WIRTSCHAFT, 1998, S. $20 \mathrm{f}$. 
Das mit Hilfe der oben beschriebenen Deckungsbeitragsrechnung ermittelte finanzielle Ergebnis des jeweiligen Produktes, bei dem die Kostenführung besteht, dient als Grundlage für die Ermittlung der Platzierungen 1 bis 4. Damit stellt sich das Vorgehen in dieser Untersuchung folgendermaßen dar:

1. In jeder Vierergruppe werden für alle Perioden die produktspezifischen Deckungsbeiträge der drei Produkte für alle vier Wettbewerber errechnet. Für jedes Produkt erhält man folglich vier Deckungsbeiträge je Periode.

2. Die vier Produktdeckungsbeiträge der vier Konkurrenten werden nach ihrer Höhe sortiert, wodurch sich die Platzierung der vier Unternehmen in Bezug auf das jeweils betrachtete Produkt ergibt. Das Unternehmen, das den höchsten Deckungsbeitrag erwirtschaftet hat, erzielt den 1. Platz usw..

3. Der Kostenführer des jeweils betrachteten Produktes ist zuvor auf der Basis der variablen Produktionskosten je Stück der 2. Periode bestimmt worden. Im dritten Schritt wird die Platzierung ermittelt, die der Kostenführer des jeweiligen Produktes bei diesem Produkt erreicht hat.

4. Die Platzierung eines Kostenführers bei einem bestimmten Produkt ist nun vergleichbar mit der Platzierung anderer Kostenführer, die in anderen Vierergruppen spielen, in der sich bspw. die Gewinnniveaus völlig unterschiedlich entwickelt haben.

5. Die pro Periode zu erfassenden Daten nehmen folglich nur die Werte 1, 2, 3 oder 4 an. Sie spiegeln den wirtschaftlichen Erfolg wider, den ein Kostenführer innerhalb seiner Vierergruppe mit dem Produkt erwirtschaftet, bei dem er die Kostenführung inne hat.

Damit basiert das Bewertungssystem in dieser Studie in seinen Grundzügen auf den gleichen Parametern, wie im offenen MARGA-Planspielwettbewerb, nämlich auf dem finanziellen Erfolg, der sich aus vielen Einzelentscheidungen ergibt. Der einzige Unterschied besteht darin, dass in der Studie der Erfolg produktweise, streng nach dem Verursachungsprinzip, ermittelt wird. $\mathrm{Da}$ das $\mathrm{Be}-$ wertungskriterium für den Erfolg das Handeln der Entscheidungsträger bestimmt, muss es, wie im vorliegenden Fall, gleichgerichtet sein. Nur so ist sicher gestellt, dass die Teams ihr gesamtes MARGA-Unternehmen nach dem gleichen erfolgmaximierenden Zielsystem steuern. Das ist die Voraussetzung dafür, dass die gefällten Entscheidungen der Teams aussagefähig und im Rahmen dieser Studie interpretierbar sind. 
Die Teilnehmer beider Planspielwettbewerbe wissen nicht, dass ihre Entscheidungen im Rahmen einer empirischen Studie erfasst und analysiert werden. Da Versuchspersonen, die sich der Teilnahme an einem Experiment bewusst sind, oft anders entscheiden, gerade weil sie sich beobachtet fühlen, wird diese Störgröße hier vollständig ausgeschaltet. Erst nach der 6 . Periode erhalten die Teams einen Fragebogen, aus dem sie die Information entnehmen können, dass ihre Entscheidungsdaten in den Perioden 1 bis 6 für ein wissenschaftliches Experiment anonym erfasst und ausgewertet werden.

Nachdem nun das Instrumentarium und die Versuchsanordnung auf die inhaltlichen Erfordernisse ausgerichtet worden sind, werden im weiteren Verlauf der Ausführungen die einzelnen Hypothesen formuliert.

\section{II.8.2 Hypothesen}

Der Ausgangspunkt der Hypothesenformulierung ist die im 5. Kapitel, Abschnitt 1.5.2, hergeleitete Grundhypothese: Die Kenntnis der Stärken und Schwächen auf Kostenebene, welche durch eine Kostenbetrachtung in der Konkurrenzanalyse abgeleitet werden können, führt insbesondere bei Kostenführern zu einer Zunahme an Planungs- und Entscheidungssicherheit und beeinflusst damit strategische Entscheidungen und den wirtschaftlichen Unternehmenserfolg.

Die Grundhypothese wird im Folgenden in sechs Einzelhypothesen zerlegt. Durch die Betrachtung einzelner Gesichtspunkte wird die Komplexität der Grundhypothese reduziert, so dass eine differenzierte, quasi in kleinen Schritten durchgeführte Untersuchung erfolgen kann.

Die Grundsatzentscheidung für eine Strategie der Kostenführerschaft, die sich für die MARGA-Unternehmen mit den niedrigsten variablen Produktionskosten je Stück, also den Kostenführern, anbietet, ist mit bestimmten Folgeentscheidungen verbunden. ${ }^{368}$ Durch die Analyse dieser Folgeentscheidungen ist es möglich, die tatsächliche Operationalisierung der Kostenführerstrategie durch die betrachteten Teams zu überprüfen. Damit kann untersucht werden, ob

368 Vgl. 1. Kapitel, Abschnitt I.1.3.3, 5. Kapitel, Abschnitt 1.5.2 und 7. Kapitel, Abschnitt II.7.3. 
Kostenführer der Untersuchungsmenge, die über ihre günstige Kostenposition informiert sind, eher dazu neigen, die Kostenführerstrategie zu verfolgen, als Kostenführer der Kontrollmenge, die ihre günstige Kostenposition nicht kennen.

Diesem Vorgehen folgend werden in den Hypothesen 1 bis 5 ausschließlich strategische Entscheidungen von Kostenführern betrachtet. Die Entscheidungsdaten anders positionierter Teams sind in den Datensätzen nicht enthalten. Die Folgeentscheidungen einer Kostenführerstrategie werden folgendermaßen in den einzelnen Hypothesen betrachtet:

Hypothese 1: Das Investitionsverhalten

Hypothese 2: Die Produktionsmengenpolitik

Hypothese 3: Die Preispolitik

Hypothese 4: Die Forschungs- und Entwicklungspolitik

Hypothese 5: Die Werbestrategie

Während die Hypothesen 1 und 2 eher den Produktionsbereich fokussieren, steht in den Hypothesen 3, 4 und 5 das Marketing im Vordergrund. Beide Aspekte sind „zwei Seiten einer Medaille“ und im Falle einer Kostenführerstrategie untrennbar miteinander verbunden: Einerseits das aggressive Marketing, durch das Marktanteile hinzu gewonnen werden sollen, um so bei einer Ausweitung der Produktionsmenge von Skalen- und Erfahrungskurveneffekten profitieren zu können. Aggressives Marketing lässt sich im Planspiel MARGA durch niedrige Preise, hohe Forschungs- und Entwicklungsaufwendungen, sowie durch eine Ausweitung des Werbebudgets erreichen, was in den Hypothesen 3, 4 und 5 betrachtet wird. ${ }^{369}$ Andererseits werden sich die Kostenführer bei einem aggressiven Marketing für eine entsprechend expansive Mengenpolitik entscheiden, die bei MARGA mit einem Kapazitätsaufbau und einer erhöhten Produktionsmenge einhergeht, was in den Hypothesen 1 und 2 überprüft wird.

369 Die vierte Möglichkeit für die Teams, die Wirkung des Marketings bei MARGA zu beeinflussen, ist die Intensität des Vertriebs, die durch die Höhe des Vertriebs-Budgets gesteuert wird. Da der Vertrieb bei MARGA nur marktweise, d.h. für alle drei Produkte eines Marktes gemeinsam, und nicht produktweise entschieden werden kann, lässt sich die Wirkung des Vertriebs den Produkten nicht verursachungsgerecht zuweisen. Dadurch können sich nicht kalkulierbare Ungenauigkeiten bezüglich der zu untersuchenden Ursache-Wirkungs-Zusammenhänge ergeben. Aus diesem Grund wird im Rahmen der vorliegenden Untersuchung das Entscheidungsverhalten der Teams nur bei drei der vier Marketing-Instrumente analysiert. 
Beide Aspekte bedingen sich gegenseitig: Ein aggressives Marketing erfordert eine Lieferfähigkeit, die diesem Marketing entspricht. Hingegen bedingt der Aufbau von Kapazitäten - und damit die Schaffung der Lieferfähigkeit - zur wirtschaftlich notwendigen Auslastung ein entsprechendes Wachstum der Nachfrage, was wiederum durch ein aggressives Marketing beeinflusst werden kann. Da die fünf Hypothesen beide Seiten der "Medaille" beleuchten, kann so durch die Betrachtung der Folgeentscheidungen die Realisierung einer Kostenführerstrategie aus unterschiedlichen Perspektiven belegt werden.

In der 6. Hypothese steht der wirtschaftliche Unternehmenserfolg im Mittelpunkt. ${ }^{370}$ Dabei werden wiederum ausschließlich die kostenführenden Teams der Untersuchungs- und der Kontrollgruppe betrachtet, die bereits zuvor in den Hypothesen 1 bis 5 fokussiert wurden. Der wirtschaftliche Unternehmenserfolg ist die Folge der zuvor betrachteten strategischen Entscheidungen. Insofern wird in Hypothese 6 untersucht, ob die Kenntnis von Stärken auf der Kostenebene die Entscheidungsträger dazu bewegt, erfolgreiche Strategien zu finden und zu verfolgen.

Im Folgenden werden alle sechs Hypothesen einzeln hergeleitet. Dabei werden zuerst die in der jeweiligen Hypothese enwarteten Sachverhalte und Zusammenhänge beschrieben. Anschließend werden Annahmen formuliert, mit deren Hilfe die Erwartungen schrittweise untersucht werden können. Auf der Basis dieser inhaltlichen Vorüberlegungen wird die Nullhypothese aufgestellt, die wiederum den Ausgangspunkt bei der Durchführung der mathematisch-statistischen Verfahren im 10. Kapitel bildet.

Bei der folgenden detaillierten Darstellung der einzelnen Hypothesen wird u.a. das jeweils erfasste Datenmaterial beschrieben. Die Beschaffenheit des Datenmaterials und eventuell vorhandene Abhängigkeiten zwischen bestimmten Datenmengen werden an späterer Stelle maßgebend bei der Auswahl der statistischen Testverfahren sein und müssen daher vorher betrachtet werden. 


\section{II.8.2.1 Hypothese 1: Strategisches Investitionsverhalten}

In der 1. Hypothese wird das strategische Investitionsverhalten der Kostenführer betrachtet, wobei diese, entsprechend den Ausführungen in Abschnitt II.8.1, entweder der Untersuchungs- oder der Kontrollmenge zugeteilt werden.

Eine expansive Investitionspolitik stellt bei MARGA die Voraussetzung für die Realisierung einer Kostenführerstrategie dar. In einem ersten Schritt soll untersucht werden, ob das Investitionsverhalten der Teams von deren Kenntnis über die eigene Kostenposition beeinflusst wird. Werden dabei Unterschiede zwischen dem Investitionsverhalten der Untersuchungs- und der Kontrollmenge festgestellt, soll weiterhin analysiert werden, worin diese Unterschiede bestehen. Die 1. Hypothese lässt sich in zwei Annahmen 1.a) und 1.b) aufgliedern:

1.a) Die Teams der Untersuchungs- und der Kontrollmenge, die sich alle in kostenführender Position befinden, zeigen in den Perioden 1 und 2 kein signifikant unterschiedliches Investitionsverhalten. Erst in den Folgeperioden, nachdem die Teams der Untersuchungsmenge über ihre günstige Kostenposition informiert worden sind, lassen sich Unterschiede feststellen.

1.b) Wenn sich ein unterschiedliches Investitionsverhalten zwischen der Untersuchungs- und der Kontrollmenge nachweisen lässt, werden die Kostenführer in der Untersuchungsmenge nach der 2. Periode die Kapazitäten in stärkerem Maße ausbauen, als die Kostenführer in der Kontrollmenge, die über ihre ebenfalls günstige Kostenposition nicht informiert sind.

Zur Überprüfung dieser Annahmen werden die Kapazitätseinheiten je Maschinentyp erfasst, die den jeweiligen Kostenführern zur Verfügung stehen. Dabei wird nur der Maschinentyp betrachtet, der für die Produktion des Kostenführerproduktes benötigt wird, d.h. Maschinentyp A, falls die Kostenführerschaft bei Produkt 1 besteht, $B$ für das Produkt 2 und Maschinentyp $C$ für das Produkt 3. Durch die Erfassung der Kapazitätseinheiten, die in jeder Planspiel-Periode erfolgt, werden sowohl Neu-, als auch Re- und Desinvestitionen berücksichtigt.

Die erfassten Kapazitätseinheiten sind an einzelne Maschinen gebunden, die jeweils über eine bestimmte Kapazität verfügen, die von der Planspielleitung in 
der Ausgangssituation festgelegt ist. So kann bspw. für die Produktion des Produktes 1 die Kapazität des Maschinentyps A nur durch den Kauf einer Maschine erhöht werden, die einheitlich über 2,2 Mio. Kapazitätseinheiten verfügt. Da dieser Wert von den Teams nicht beeinflussbar ist, können die Kapazitäten nur in vorgegebenen Schritten auf- oder abgebaut werden. Außerdem kann nur eine begrenzte Anzahl von Maschinen gekauft werden. Folglich kann das Merkmal "Kapazitätseinheiten" in dieser Untersuchung nur eine endliche Anzahl an Werten annehmen und ist somit als diskretes Merkmal anzusehen. Das zugrundeliegende Skalenniveau ist metrisch. ${ }^{371}$

Weil die Datenerhebung für beide Planspiele MARGA und THE BOARD, sowie für alle drei Produkte getrennt erfolgt, kann die Überprüfung der Hypothese 1 insgesamt sechs Mal durchgeführt werden. Tabelle 6 gibt einen Überblick über die Anzahl der zur Untersuchung zugelassenen Datensätze je Planspiel und Produkt, sowie unterschieden nach Untersuchungs- und Kontrollmenge. ${ }^{372}$

\begin{tabular}{|l|l|c|c|c|}
\cline { 3 - 5 } \multicolumn{2}{c|}{} & $\begin{array}{c}\text { Untersu- } \\
\text { chungsmenge }\end{array}$ & $\begin{array}{c}\text { Kontroll- } \\
\text { menge }\end{array}$ & $\begin{array}{c}\text { Datensätze } \\
\text { insgesamt }\end{array}$ \\
\hline \multirow{3}{*}{ MARGA } & Produkt 1 & 36 & 35 & 71 \\
\cline { 2 - 5 } & Produkt 2 & 36 & 35 & 71 \\
\cline { 2 - 5 } & Produkt 3 & 36 & 35 & 71 \\
\hline \multirow{3}{*}{ THE BOARD } & Produkt 1 & 59 & 54 & 113 \\
\cline { 2 - 5 } & Produkt 2 & 56 & 57 & 113 \\
\cline { 2 - 5 } & Produkt 3 & 58 & 55 & 113 \\
\hline
\end{tabular}

Tabelle 6: Hypothese 1: Die zugelassenen Datensätze

Während bei MARGA alle 71 Datensätze gewertet werden konnten, mussten bei THE BOARD von 118 Datensätzen je Produkt jeweils fünf Teams ausgeschlossen werden, da sie nicht den Marktforschungsbericht "vier" geordert hatten und somit die Bedingung der Informationsgleichheit verletzt worden ist. Beim Produkt 1 betraf dies ausschließlich Teams, die der Kontrollmenge zugeordnet waren. Beim Produkt 2 musste die Untersuchungsmenge um vier und die Kontrollmenge um ein Team reduziert werden und beim Produkt 3 waren zwei Teams der Untersuchungs- und drei Teams aus der Kontrollmenge betroffen. Diese Struktur der zugelassenen Datensätze kann auf die Hypothesen

371 Vgl. HARTUNG/ELPELt, 1999, S. $18 \mathrm{f}$.

372 Die Ausschlusskriterien dieser Untersuchung werden im 8. Kapitel, Abschnitt II.8.1, erläutert. 
1, 2, 4 und 6 angewendet werden, bei denen keine Einzelbetrachtung der vier Marktsegmente erfolgt.

Zwischen den Datensätzen beider Planspiele und zwischen den Daten der Untersuchungs- und der Kontrollmenge bestehen keine Abhängigkeiten, da diese aus Entscheidungen der kostenführenden Teams abgeleitet werden, die zu keinem Zeitpunkt Informationen über die Entscheidungen anderer Teams in ihrer oder in anderen Gruppen haben.

Zusammenfassend lässt sich die Nullhypothese $H_{0} 1$ ) formulieren, die den Ausgangspunkt der später folgenden mathematisch-statistischen Testverfahren darstellt:

Die Teams der Untersuchungs- und der Kontrollmenge, die sich alle in kostenführender Position befinden, zeigen in den einzelnen Perioden der Untersuchung kein signifikant unterschiedliches Investitionsverhalten, d.h. beide Teammengen verfügen über ähnlich viele Kapazitätseinheiten.

\section{II.8.2.2 Hypothese 2: Produktionsmengenpolitik}

Die Unternehmen, die eine expansive Mengenstrategie verfolgen, profitieren von Skalen- und Erfahrungskurveneffekten, die dazu beitragen, die Kostenposition weiter $\mathrm{zu}$ verbessern. ${ }^{373}$ Aus diesem Grund wird von den Teams der Untersuchungsmenge bei der Umsetzung der Kostenführerstrategie - in Verbindung mit einem vergleichsweise aggressiven Marketing - eine expansive Produktionsmengenpolitik erwartet. In der 2. Hypothese wird untersucht, ob Informationen über die Kostenposition einen Einfluss auf die Produktionsstrategie hinsichtlich der Menge haben. In diesem Fall sollten sich Unterschiede in den Produktionsmengen zwischen den kostenführenden Teams ergeben, je nachdem, ob sie der Untersuchungs- oder der Kontrollmenge zugeteilt sind. Die 2. Hypothese soll wiederum in zwei Einzelannahmen 2.a) und 2.b) gegliedert werden:

373 Vgl. 1. Kapitel, Abschnitt I.1.3.3, 5. Kapitel, Abschnitt I.5.2 und 7. Kapitel, Abschnitt II.7.3. 
2.a) Die Teams der Untersuchungs- und der Kontrollmenge weisen hinsichtlich der Produktionsmengenstrategie in den Perioden 1 und 2 keine signifikanten Unterschiede auf. Erst nach der Periode 2, nachdem die Teams der Untersuchungsmenge Informationen über ihre günstige Kostenposition erhalten haben, lassen sich Unterschiede nachweisen.

2.b) Wenn sich Unterschiede hinsichtlich der Produktionsmengenstrategie beider Teammengen zeigen, wird enwartet, dass die Kostenführer der Untersuchungsmenge nach der 2. Periode eine expansivere Politik verfolgen, als die Kostenführer der Kontrollmenge, die nicht über ihre günstige Kostenposition informiert sind.

Zur Überprüfung der Annahmen wird die prozentuale Abweichung der kumulierten Produktionsmenge des zu betrachtenden Kostenführers von der durchschnittlichen kumulierten Produktionsmenge aller vier Marktteilnehmer seiner Gruppe erfasst. Da die vorliegenden Daten die jeweiligen prozentualen Abweichungen vom Durchschnitt angeben, lassen sich Aussagen darüber treffen, wie deutlich Entscheidungen vom Durchschnitt abweichen, d.h. wie stark die Ausprägung einer Strategie ist bzw. mit welchem Nachdruck oder mit welcher Deutlichkeit sie verfolgt wird. Dieser Zusammenhang kommt durch einen entsprechend hohen positiven oder negativen Prozentwert zum Ausdruck.

Im Gegensatz zur Hypothese 1, bei der das Merkmal nur eine endliche Anzahl von Werten annehmen kann und damit eine diskrete Ausprägung zeigt, kann das hier betrachtete Untersuchungsmerkmal theoretisch unendlich viele Werte annehmen. Die Merkmalsausprägung ist damit stetig und weist eine metrische Skalierung auf. 374

Tabelle 7 gibt - gemäß den Ausführungen zur Hypothese 1 - einen Überblick über die Anzahl der zur Untersuchung der 2. Hypothese zugelassenen Datensätze je Planspiel und Produkt. ${ }^{375}$ Dabei wird nach Untersuchungs- und Kontrollmenge unterschieden. Es ergeben sich insgesamt sechs Untersuchungsreihen, für die Produkte 1, 2 und 3 bei MARGA und bei THE BOARD.

374 Vgl. HARTUNG/ELPELT, 1999, S. $18 \mathrm{f}$.

375 Die Ausschlusskriterien dieser Untersuchung werden im 8. Kapitel, Abschnitt II.8.1, erläutert. 


\begin{tabular}{|l|c|c|c|c|}
\cline { 3 - 5 } \multicolumn{2}{c|}{} & $\begin{array}{c}\text { Untersu- } \\
\text { chungsmenge }\end{array}$ & $\begin{array}{c}\text { Kontroll- } \\
\text { menge }\end{array}$ & $\begin{array}{c}\text { Datensätzeins } \\
\text { gesamt }\end{array}$ \\
\hline \multirow{3}{*}{ MARGA } & Produkt 1 & 36 & 35 & 71 \\
\cline { 2 - 5 } & Produkt 2 & 36 & 35 & 71 \\
\cline { 2 - 5 } & Produkt 3 & 36 & 35 & 71 \\
\hline THE BOARD & Produkt 1 & 59 & 54 & 113 \\
\cline { 2 - 5 } & Produkt 2 & 56 & 57 & 113 \\
\cline { 2 - 5 } & Produkt 3 & 58 & 55 & 113 \\
\hline
\end{tabular}

Tabelle 7: Hypothese 2: Die zugelassenen Datensätze

Auch bei der 2. Hypothese bestehen weder zwischen den Datensätzen beider Planspiele, noch zwischen den Daten der Untersuchungs- und der Kontrollmenge Abhängigkeiten, da die Daten aus Entscheidungen von Teams abgeleitet werden, die keine Informationen über die Entscheidungen anderer Teams in anderen Gruppen haben.

Aus den Vorüberlegungen ergibt sich folgende Nullhypothese $\mathrm{H}_{0} 2$ ):

Die Teams der Untersuchungs- und der Kontrollmenge, die sich alle in kostenführender Position befinden, weisen hinsichtlich der Produktionsmengenstrategie in den einzelnen Perioden der Untersuchung keine signifikanten Unterschiede auf, d.h. die prozentuale Abweichung der kumulierten Produktionsmenge des zu betrachtenden Kostenführers von der durchschnittlichen kumulierten Produktionsmenge aller vier Konkurrenten seiner Gruppe wird bei den Teams der Untersuchungs- und der Kontrollmenge ähnlich sein.

\subsubsection{Hypothese 3: Preispolitik}

Die Ausweitung der Produktionsmenge im Rahmen einer Kostenführerstrategie muss durch ein entsprechend aggressives Marketing begleitet werden, das zu einem Wachstum auf der Absatzseite führt. Der Preis stellt in diesem Zusammenhang ein wesentliches Marketinginstrument dar, das besonders von Kostenführern genutzt werden kann. ${ }^{376}$ In der 3. Hypothese wird untersucht, ob die Kenntnis der Kostenposition einen Einfluss auf die Wahl der Preisstrategie

376 Vgl. 1. Kapitel, Abschnitt I.1.3.3, 5. Kapitel, Abschnitt I.5.2 und 7. Kapitel, Abschnitt II.7.3. 
hat. Trifft dies zu, sollten Unterschiede in der Preisstrategie der Teams der Untersuchungs- und der Kontrollmenge nachweisbar sein. Wiederum werden für die Hypothese 3 zwei Einzelannahmen 3.a) und 3.b) formuliert:

3.a) Die Teams der Untersuchungs- und der Kontrollmenge weisen hinsichtlich inrer Preisstrategie in den Perioden 1 und 2 keine signifikanten Unterschiede auf. Erst nach der Periode 2, nachdem die Teams der Untersuchungsmenge Informationen über ihre kostenführende Position erhalten haben, lassen sich Unterschiede feststellen.

3.b) Für den Fall, dass sich nach der 2. Periode eine unterschiedliche Preisstrategie zwischen den Teams der Untersuchungs- und der Kontrollmenge nachweisen lässt, werden die Teams der Untersuchungsmenge ein niedrigeres Preisniveau aufweisen, als die Teams der Kontrollmenge, die keine Informationen über ihren Kostenvorsprung haben.

Zur Überprüfung der 3. Hypothese wird die prozentuale Abweichung des Preises des Kostenführers vom Durchschnittspreis aller vier Konkurrenten erfasst. Die vorliegenden Daten entsprechen den jeweiligen prozentualen Abwiechungen vom Branchendurchschnitt, so dass daraus, wie in Hypothese 2, auf die Stärke der Ausprägung einer Strategie zurückgeschlossen werden kann. Darüber hinaus wird durch die Verwendung dieser Kennzahl eine Unabhängigkeit vom absoluten Preisniveau einzelner Vierergruppen erreicht. Dieses bildet sich in jeder Planspiel-Gruppe unterschiedlich heraus und muss daher im Falle einer Untersuchung, die Teams mehrerer Vierergruppen einbezieht, vergleichbar gemacht werden.

Wie zuvor in Hypothese 2 kann das betrachtete Merkmal in der 3. Hypothese theoretisch unendlich viele Werte annehmen. Auch hier ist das Untersuchungsmerkmal damit stetig. Es weist eine metrische Skalierung auf.

Die Daten werden bei MARGA und bei THE BOARD für jedes der zwölf Marktsegmente, d.h. für drei Produkte auf vier Märkten erhoben, so dass insgesamt 24 einzelne Experimente durchgeführt werden können. Tabelle 8 gibt einen Überblick über die Anzahl der zur Untersuchung zugelassenen Datensätze. ${ }^{377}$

377 Die Ausschlusskriterien dieser Untersuchung werden im 8. Kapitel, Abschnitt II.8.1, erläutert. Christoph Heinen - 978-3-631-75315-6 
Da die Hypothesen 3 und 5 für jedes Marktsegment einzeln betrachtet werden, müssen hier zusätzlich die Datensätze von der Untersuchung ausgeschlossen werden, bei denen die Kostenführer aus Marktsegmenten ausgestiegen sind, bei denen sie die Kostenführung inne hatten. Dies betrifft drei Fälle, die in Tabelle 8 in fetter Schrift dargestellt und in der weiteren Untersuchung entsprechend berücksichtigt werden.

\begin{tabular}{|l|l|c|c|c|c|c|c|c|c|}
\cline { 3 - 11 } \multicolumn{2}{c|}{} & \multicolumn{3}{c|}{ Untersuchungsmenge } & \multicolumn{4}{c|}{ Kontrollmenge } \\
\cline { 3 - 11 } & M1 & M2 & M3 & M4 & M1 & M2 & M3 & M4 \\
\hline MARGA & Prod. 1 & 36 & 36 & 36 & 36 & 35 & 35 & 35 & 35 \\
\cline { 2 - 11 } & Prod. 2 & 36 & 36 & 36 & 36 & 35 & 35 & 35 & 35 \\
\cline { 2 - 10 } & Prod. 3 & 36 & 36 & 36 & 36 & 35 & 35 & 35 & 35 \\
\hline THE BOARD & Prod. 1 & 59 & 59 & 59 & 59 & 54 & 54 & 54 & 54 \\
\cline { 2 - 10 } & Prod. 2 & 56 & 56 & 56 & $\mathbf{5 5}$ & 57 & 57 & $\mathbf{5 6}$ & 57 \\
\cline { 2 - 10 } & Prod. 3 & 58 & 58 & $\mathbf{5 7}$ & $\mathbf{5 8}$ & 55 & 55 & 55 & 55 \\
\hline
\end{tabular}

Tabelle 8: Hypothese 3: Die zugelassenen Datensätze

Wie zuvor besteht zwischen den Datensätzen Unabhängigkeit.

Abschließend soll folgende Nullhypothese $\mathrm{H}_{0} 3$ ) aufgestellt werden:

Die Teams der Untersuchungs- und der Kontrollmenge, die sich alle in kostenführender Position befinden, zeigen hinsichtlich ihrer Preisstrategie in den einzelnen Perioden keine signifikanten Unterschiede. Die prozentuale Abweichung des Preises des Kostenführers vom Durchschnittspreis aller vier Konkurrenten wird daher in beiden Mengen nur gering voneinander abweichen.

\section{II.8.2.4 Hypothese 4: Forschungs- und Entwicklungspolitik}

Im Planspiel MARGA wird durch die Höhe des F\&E-Budgets die Qualität des Produktes im Rahmen der Marketing-Strategie definiert. In der 4. Hypothese wird untersucht, ob die Kenntnis der Kostenposition einen Einfluss auf die Strategie hat, die im F\&E-Bereich bei MARGA durchgeführt wird. In diesem Fall sollten sich Unterschiede in der F\&E-Strategie zwischen den Teams der Untersuchungs- und der Kontrollmenge ergeben. Zur 4. Hypothese werden zwei Annahmen 4.a) und 4.b) formuliert: 
4.a) Die Teams der Untersuchungs- und der Kontrollmenge weisen in den Perioden 1 und 2 keine signifikanten Unterschiede bezüglich ihrer F\&EStrategie auf. Erst nach der 2. Periode, nachdem die Teams der Untersuchungsmenge Informationen über ihre günstige Kostenposition erhalten haben, sollten Unterschiede feststellbar sein.

4.b) Falls nach der 2. Periode Unterschiede in der F\&E-Strategie zwischen den Teams der Untersuchungs- und der Kontrollmenge nachgewiesen werden können, sollten diese in einem erhöhten F\&E-Budget bei den Kostenführern der Untersuchungsmenge bestehen.

Zur Untersuchung dieser Annahmen wird die prozentuale Abweichung des kumulierten F\&E-Budgets des Kostenführers vom durchschnittlichen kumulierten F\&E-Budget aller Konkurrenten erfasst. Durch die Ermittlung der prozentualen Abweichung vom Durchschnitt lassen sich Aussagen darüber treffen, wie extrem Entscheidungen vom Durchschnitt abweichen, was durch einen entsprechend hohen positiven oder negativen Prozentwert zum Ausdruck kommt.

Wie in den Hypothesen 2 und 3 kann durch die Erfassung prozentualer Abweichungen das Merkmal theoretisch unendlich viele Werte annehmen. Damit ist die Merkmalsausprägung stetig. Sie weist eine metrische Skalierung auf. Auch bei der 4. Hypothese besteht zwischen den Daten Unabhängigkeit.

Insgesamt können sechs einzelne Experimente durchgeführt werden. Tabelle 9 gibt - entsprechend den Ausführungen zur Hypothese 1 - einen Überblick über die Anzahl der zur Untersuchung zugelassenen Datensätze:378

\begin{tabular}{|l|c|c|c|c|}
\cline { 3 - 5 } \multicolumn{2}{c|}{} & $\begin{array}{c}\text { Untersu- } \\
\text { chungsmenge }\end{array}$ & $\begin{array}{c}\text { Kontroll- } \\
\text { menge }\end{array}$ & $\begin{array}{c}\text { Datensätze } \\
\text { insgesamt }\end{array}$ \\
\hline MARGA & Produkt 1 & 36 & 35 & 71 \\
\cline { 2 - 5 } & Produkt 2 & 36 & 35 & 71 \\
\cline { 2 - 5 } & Produkt 3 & 36 & 35 & 71 \\
\hline THE BOARD & Produkt 1 & 59 & 54 & 113 \\
\cline { 2 - 5 } & Produkt 2 & 56 & 57 & 113 \\
\cline { 2 - 5 } & Produkt 3 & 58 & 55 & 113 \\
\hline
\end{tabular}

Tabelle 9: Hypothese 4: Die zugelassenen Datensätze

378 Die Ausschlusskriterien dieser Untersuchung werden im 8. Kapitel, Abschnitt 11.8.1, erläutert. Christoph Heinen - 978-3-631-75315-6 
Folgende Nullhypothese $\mathrm{H}_{0} 4$ ) wird zur Überprüfung der Aussagen formuliert:

Die Teams der Untersuchungs- und der Kontrollmenge, die sich alle in kostenführender Position befinden, weisen in den einzelnen Perioden der Untersuchung keine signifikanten Unterschiede bezüglich ihrer F\&E-Strategie auf. Die prozentuale Abweichung des kumulierten F\&E-Budgets des Kostenführers vom durchschnittlichen kumulierten F\&E-Budget aller Konkurrenten wird daher in der Untersuchungs- und in der Kontrollmenge ähnlich sein.

\section{II.8.2.5 Hypothese 5: Werbestrategie}

Der Aggressivitätsgrad der Werbung wird bei MARGA durch die Höhe des Werbebudgets festgelegt. Die Strategie der Kostenführerschaft ist, wie bereits mehrfach dargelegt ${ }^{379}$, mit einer Ausweitung der Produktionsmengen verbunden und wird von einem vergleichsweise aggressiven Marketing begleitet. Dieses lässt sich bei MARGA durch niedrige Preise, hohe Produktqualität und ein hohes Werbebudget im Vergleich zu den Wettbewerbern realisieren.

In der 5. Hypothese wird untersucht, ob die Kenntnis der Kostenposition einen Einfluss auf die Werbeaufwendungen hat. In diesem Fall sollten sich Unterschiede im Werbebudget zwischen der Untersuchungs- und der Kontrollmenge ergeben. Für die 5. Hypothese werden zwei Annahmen 5.a) und 5.b) formuliert:

5.a) Die Teams der Untersuchungs- und der Kontrollmenge weisen in den Perioden 1 und 2 keine signifikanten Unterschiede bezüglich ihrer Werbeaufwendungen auf. Erst nach der 2. Periode, nachdem die Teams der Untersuchungsmenge Informationen über ihre günstige Kostenposition erhalten haben, sollten Unterschiede feststellbar sein.

5.b) Falls nach der 2. Periode Unterschiede in den Werbeaufwendungen zwischen den Teams der Untersuchungs- und der Kontrollmenge nachgewiesen werden können, sollten diese darin bestehen, dass die Teams der Untersuchungsmenge ein höheres Werbebudget einsetzen, als die Teams der Kontrollmenge, die keine Informationen über ihre günstige Kostenposition erhalten.

${ }^{379}$ Vgl. 1. Kapitel, Abschnitt I.1.3.3, 5. Kapitel, Abschnitt I.5.2 und 7. Kapitel, Abschnitt II.7.3. 
Zur Untersuchung dieser Annahmen wird für alle Perioden die prozentuale Abweichung des kumulierten Werbebudgets des Kostenführers vom durchschnittlichen kumulierten Werbebudget aller Konkurrenten erfasst. Durch die Ermittlung der prozentualen Abweichung vom Durchschnitt lassen sich Aussagen darüber treffen, wie extrem Entscheidungen vom Durchschnitt abweichen, was durch einen entsprechend hohen positiven oder negativen Prozentwert zum Ausdruck kommt. Die Merkmalsausprägung ist damit wie in den Hypothese 2, 3 und 4 stetig. Sie ist ebenfalls metrisch skaliert.

Die Daten werden bei MARGA und bei THE BOARD für jedes der zwölf Marktsegmente, d.h. für drei Produkte auf vier Märkten erhoben, so dass insgesamt 24 einzelne Experimente durchgeführt werden. Tabelle 10 gibt - entsprechend den Ausführungen zur Hypothese 3 - einen Überblick über die Anzahl der zur Untersuchung zugelassenen Datensätze: 380

\begin{tabular}{|l|l|l|c|c|c|c|c|c|c|}
\cline { 3 - 10 } \multicolumn{2}{c|}{} & \multicolumn{3}{|c|}{ Untersuchungsmenge } & \multicolumn{4}{c|}{ Kontrollmenge } \\
\cline { 3 - 11 } \multicolumn{2}{c|}{} & M1 & M2 & M3 & M4 & M1 & M2 & M3 & M4 \\
\hline \multirow{4}{*}{ MARGA } & Prod. 1 & 36 & 36 & 36 & 36 & 35 & 35 & 35 & 35 \\
\cline { 2 - 11 } & Prod. 2 & 36 & 36 & 36 & 36 & 35 & 35 & 35 & 35 \\
\cline { 2 - 10 } & Prod. 3 & 36 & 36 & 36 & 36 & 35 & 35 & 35 & 35 \\
\hline THE BOARD & Prod. 1 & 59 & 59 & 59 & 59 & 54 & 54 & 54 & 54 \\
\cline { 2 - 10 } & Prod. 2 & 56 & 56 & 56 & 55 & 57 & 57 & 56 & 57 \\
\cline { 2 - 10 } & Prod. 3 & 58 & 58 & 57 & 58 & 55 & 55 & 55 & 55 \\
\hline
\end{tabular}

Tabelle 10: Hypothese 5: Die zugelassenen Datensätze

Auch für die 5. Hypothese gilt die bereits erläuterte Unabhängigkeit der Daten.

Folgende Nullhypothese $\mathrm{H}_{0} 5$ ) wird zur Überprüfung der Aussagen aufgestellt:

Die Teams der Untersuchungs- und der Kontrollmenge, die sich alle in kostenführender Position befinden, weisen in den einzelnen Perioden keine signifikanten Unterschiede bezüglich ihrer Werbeaufwendungen auf. Die prozentuale Abweichung des kumulierten Werbebudgets des Kostenführers vom durchschnittlichen kumulierten Werbebudget aller Konkurrenten wird damit in der Untersuchungs- und in der Kontrollmenge ähnlich sein.

380 Die Ausschlusskriterien dieser Untersuchung werden im 8. Kapitel, Abschnitt II.8.1, erläutert. Christoph Heinen - 978-3-631-75315-6 


\subsubsection{Hypothese 6: Wirtschaftlicher Erfolg}

In der 6. Hypothese wird der wirtschaftliche Unternehmenserfolg ${ }^{381}$ untersucht. Dabei ist zu beachten, dass die Kenntnis der eigenen Kostenposition nur das Handeln beeinflussen kann, das in den Hypothesen 1 bis 5 untersucht wird, nicht jedoch das Ergebnis des Handelns, das im Erfolg zum Ausdruck kommt. Insofern wird in Hypothese 6 untersucht, ob die Kenntnis der eigenen Kostenposition die Entscheidungsträger dazu bewegt, erfolgreiche Strategien zu finden und zu verfolgen.

Der Erfolg wird bei diesem Experiment durch die Platzierung der Teams ausgedrückt, so dass sich, falls ein Einfluss besteht, Unterschiede in der Platzierung zwischen den Teams der Untersuchungs- und der Kontrollmenge nachweisen lassen sollten. In diesem Fall soll weiterhin analysiert werden, welche Teammenge die höhere Platzierung aufweisen kann. Diese Zusammenhänge werden in zwei Annahmen 6.a) und 6.b) formuliert:

6.a) Die Teams der Untersuchungs- und der Kontrollmenge weisen in den Perioden 1 und 2 keine signifikanten Unterschiede bezüglich ihrer Platzierung auf. Erst nach der 2. Periode, nachdem die Teams der Untersuchungsmenge Informationen über ihre günstige Kostenposition erhalten haben, sollten Unterschiede feststellbar sein.

6.b) Falls sich nach der 2. Periode Unterschiede in der Platzierung zwischen den Teams der Untersuchungs- und der Kontrollmenge nachweisen lassen, wird erwartet, dass die Teams der Untersuchungsmenge eine höhere Platzierung erreichen, als die Teams der Kontrollmenge, die keine Informationen über ihre kostenführende Position erhalten.

Zur Untersuchung dieser Aussagen wird die periodenaktuelle Platzierung erfasst, die auf Basis der Produktdeckungsbeiträge ermittelt wird, die in der jeweiligen Periode erwirtschaftet werden. ${ }^{382}$

381 Das hier verwandte Verständnis von „Erfolg“ wird im 8. Kapitel, Abschnitt II.8.1, erläutert.

382 Die zugrundeliegende Deckungsbeitragsrechnung wurde im 8. Kapitel, Abschnitt II.8.1, dargestellt. 
Das Merkmal „Platzierung“ kann nur die Werte 1, 2, 3 oder 4 annehmen, die dann entsprechend für den 1., 2., 3. oder 4. Platz stehen. Die Daten sind damit diskret. Sie weisen eine ordinale Skalierung auf.

Die Überprüfung der 6. Hypothese kann sechs Mal, d.h. auf der Basis beider Planspiele und für jeweils drei Produkte durchgeführt werden. Tabelle $11 \mathrm{gibt}$ einen Überblick über die Anzahl der zur Untersuchung zugelassenen Datensätze je Planspiel und Produkt, sowie unterschieden nach Untersuchungs- und Kontrollmenge: ${ }^{383}$

\begin{tabular}{|l|l|c|c|c|}
\cline { 3 - 5 } \multicolumn{2}{c|}{} & $\begin{array}{c}\text { Untersu- } \\
\text { chungsmenge }\end{array}$ & $\begin{array}{c}\text { Kontroll- } \\
\text { menge }\end{array}$ & $\begin{array}{c}\text { Datensätze } \\
\text { insgesamt }\end{array}$ \\
\hline \multirow{3}{*}{ MARGA } & Produkt 1 & 36 & 35 & 71 \\
\cline { 2 - 5 } & Produkt 2 & 36 & 35 & 71 \\
\cline { 2 - 5 } & Produkt 3 & 36 & 35 & 71 \\
\hline THE BOARD & Produkt 1 & 59 & 54 & 113 \\
\cline { 2 - 5 } & Produkt 2 & 56 & 57 & 113 \\
\cline { 2 - 5 } & Produkt 3 & 58 & 55 & 113 \\
\hline
\end{tabular}

Tabelle 11: Hypothese 6: Die zugelassenen Datensätze

Auch für die 6. Hypothese gilt die Unabhängigkeit der Daten.

Es ergibt sich folgende Nullhypothese $\left.H_{0} 6\right)$ :

Die Teams der Untersuchungs- und der Kontrollmenge, die sich alle in kostenführender Position befinden, weisen in den einzelnen Perioden keine signifikanten Unterschiede bezüglich ihrer Platzierung auf.

383 Die Ausschlusskriterien dieser Untersuchung werden im 8. Kapitel, Abschnitt 11.8.1, erläutert. 


\section{Kapitel Auswahl der statistischen Testverfahren}

Die im 8. Kapitel verbal formulierten Hypothesen sollen im weiteren Verlauf der Studie mit Hilfe mathematisch-statistischer Verfahren überprüft werden. ${ }^{384}$

Bei der Auswahl der Testverfahren spielt die Beschaffenheit des Datenmaterials eine entscheidende Rolle. Diese ist in einem ersten Schritt im vorherigen Kapitel analysiert worden, so dass nun im zweiten Schritt das entsprechende Testverfahren ausgewählt werden kann.

Bei der Auswahl der Testverfahren ist zwischen parametrischen und nichtparametrischen Tests zu unterscheiden. ${ }^{385}$ Für den Einsatz parametrischer Verfahren, wie bspw. dem oft verwendeten t-Test, ist ein Verteilungstyp der Daten anzunehmen, im Beispiel des t-Tests die Normalverteilung. ${ }^{386}$ Diese Annahme eines Verteilungstyps kann in dieser Studie nicht mit Gewissheit erfolgen, weil die vorliegenden Daten teilweise weder stetig sind, noch die Eigenschaft der Symmetrie erfüllen. Durch den Verzicht auf den Einsatz parametrischer Testverfahren wird eine falsche Testentscheidung aufgrund falscher Modellannahmen ausgeschlossen, d.h. eine Fehlklassifikation wird vermieden. Aus diesem Grund werden für die Überprüfung aller Hypothesen nichtparametrische Tests verwendet.

Der im 8. Kapitel für jede Hypothese angegebene Merkmalstyp ist mitentscheidend bei der Auswahl der einzusetzenden statistischen Verfahren. Folgende Merkmalsarten der Untersuchungseinheiten sind zu berücksichtigen:

Hypothese 1: diskretes Merkmal

Hypothese 3: stetiges Merkmal

Hypothese 5: stetiges Merkmal
Hypothese 2:stetiges Merkmal Hypothese 4:stetiges Merkmal Hypothese 6:diskretes Merkmal

Wegen der unterschiedlichen Merkmalstypen der betrachteten Untersuchungsvariablen ist es notwendig, zwei statistische Testverfahren zu verwenden. Dabei

\footnotetext{
384 Bei die Auswahl und Anwendung der statistischen Testverfahren stand dem Autor Dipl.Math. Stefan Kraft, Seminar für Wirtschafts- und Sozialstatistik der Universität zu Köln, als Gesprächspartner zur Seite.
}

$385 \mathrm{Vgl}$. GIBBONS/CHAKRABORTI, 1992, S. 3 f.

386 Vgl. BÜNING/TRENKLER, 1994, S. 39. 
kommen der Wilcoxon-Rangsummen-Test ${ }^{387}$ und der Kolmogorov-Smirnov-Anpassungstest ${ }^{388}$ zum Einsatz.

Der Kolmogorov-Smirnov-Anpassungstest wird insbesondere bei stetigen Daten angewendet ${ }^{389}$, wie sie in den Hypothesen 2, 3, 4 und 5 vorliegen, in denen prozentuale Abweichungen von einem Durchschnittswert erfasst werden. Da der Kolmogorov-Smirnov-Anpassungstest wesentlich mehr Informationen aus den Datensätzen einbezieht, wird er dem Wilcoxon-Rangsummen-Test vorgezogen. Für die Hypothesen 1 und 6 muss dagegen aufgrund der diskreten Merkmale der Wilcoxon-Rangsummen-Test verwendet werden, der im Folgenden detailliert dargestellt wird. Anschließend wird der Kolmogorov-Smirnov-Anpassungstest erläutert.

\section{II.9.1 Wilcoxon-Rangsummen-Test}

Gegeben sind zwei unabhängige Stichproben $X_{1}, X_{2}, \ldots, X_{m}$ und $Y_{1}, Y_{2}, \ldots, Y_{n}$, wobei der Gesamtumfang der Stichprobe $\mathrm{N}=\mathrm{m}+\mathrm{n}$ entspricht.

Folgende Annahmen werden getroffen:390

1. $X_{1}, X_{2}, \ldots, X_{m}$ ist eine einfache Zufallsstichprobe, die einer Grundgesamtheit 1 (Population 1) entstammt. Die einzelnen Beobachtungen sind unabhängig und identisch verteilt.

$Y_{1}, Y_{2}, \ldots, Y_{n}$ ist eine einfache Zufallsstichprobe, die einer Grundgesamtheit 2 (Population 2) entstammt. Die einzelnen Beobachtungen sind unabhängig und identisch verteilt.

2. Die Beobachtungen $X_{1}, X_{2}, \ldots, X_{m}$ und $Y_{1}, Y_{2}, \ldots, Y_{n}$ sind gemeinsam voneinander unabhängig, d.h. es liegt Unabhängigkeit innerhalb und zwischen beiden Populationen vor.

387 Vgl. zum Wilcoxon-Rangsummen-Testverfahren ausführlich: BÜNING/TRENKLER, 1994, S. 131 ff.; HARTUNG, 1999, S. 513 ff., sowie die dort angegebene Literatur.

388 Vgl. zum Kolmogorov-Smirnov-Anpassungstest ausführlich: CONOVER, 1971, S. 308 ff.; HARTUNG, 1999, S. 520 ff., sowie die dort angegebene Literatur.

389 Vgl. CONOVER, 1971, S. 48 ff.; HARTUNG, 1999, S. 522.

$390 \mathrm{Vgl}$. HOLLANDERMOLFE, 1999, S. 106. 
Die betrachtete Nullhypothese lautet: ${ }^{391}$

$H_{0}: F(x)=G(x), \forall x \in \mathbb{R}$,

wobei $F(x)$ und $G(x)$ die den Grundgesamtheiten zugrundeliegenden Verteilungsfunktionen sind, d.h.

$$
F(x)=P(X \leq x) \text { und } G(x)=P(Y \leq x)
$$

Die Verteilungsfunktionen geben demnach an, mit welcher Wahrscheinlichkeit eine Variable Werte kleiner oder gleich einem vorgegebenen Wert annimmt.

Folgender Grundgedanke liegt dem Wilcoxon-Rangsummen-Test zu Grunde:392 Sämtliche beobachteten Werte werden der Größe nach aufsteigend geordnet. Danach wird allen Werten ein Rang in dieser geordneten Stichprobe zugewiesen. Sind mehrere Werte identisch, was bei diskreten Daten möglich sein kann, wird diesen Werten ein mittlerer Rang zugewiesen. ${ }^{393}$

Stammen die beobachteten Werte aus derselben Population, wie in der Nullhypothese formuliert, so ist zu enwarten, dass weder die $x$-Werte, noch die $y$ Werte dazu tendieren, größere bzw. kleinere Werte als die jeweilige andere Variable anzunehmen. Auf diesem Grundgedanken basiert die Teststatistik des Verfahrens:

$$
W_{m, n}=\sum_{i=1}^{m} R(X)
$$

Es wird die Summe der Ränge aller $x$-Werte gebildet und die Nullhypothese abgelehnt, falls diese Summe „zu groß“ oder „zu klein“ ist. Für die Testgröße $W_{m, n}$ lässt sich für den Fall, dass keine Bindungen vorliegen, zeigen, dass sie bei Gültigkeit der betrachteten Nullhypothese asymptotisch, d.h. für Stichprobenumfänge $m, n \geq 30$, einer Normalverteilung folgt. Für die praktische Anwendung erscheint es sinnvoll, diese Testgröße in folgende Form zu überführen:394

\footnotetext{
$391 \mathrm{Vgl}$. HOLLANDER/WOLFE, 1999, S. $106 \mathrm{f}$.

392 Vgl. LeHMANN, 1975, S. $5 \mathrm{ff}$.

393 Wäre z.B. in der Stichprobe die Zahl 1 der kleinste auftretende Wert mit eine absoluten Häufigkeit von 5 , so erhält dieser Wert einen mittleren Rang von $3=(1+2+3+4+5) / 5$. $394 \mathrm{Vgl}$. Hollander/WOLFE, 1999, S. $108 \mathrm{ff}$.
} 


$$
T_{m, n}=\frac{W_{m, n}-E\left[W_{m, n}\right]}{\sqrt{\operatorname{Var}\left(W_{m, n}\right)}} \underset{\text { asy }}{\approx} N(0,1)
$$

Man spricht hier von einer sog. Standardisierung, da von der ursprünglichen Testgröße $W_{m, n}$ ihr Erwartungswert abgezogen wird und danach durch die Standardabweichung, d.h. durch die Wurzel der Varianz, dividiert wird. Für $T_{m, n}$ gilt: $E\left[T_{m, n}\right]=0$ und $\operatorname{Var}\left[T_{m, n}\right]=1$.

Die Testgröße ist für $m, n \geq 30$ asymptotisch standardnormalverteilt. Durch die Standardisierung, d.h. den Übergang zu einer Statistik mit Erwartungswert Null und Varianz Eins, bleibt die Eigenschaft der asymptotischen Normalverteilung erhalten. Da für eine Standardnormalverteilung Tabellenwerke mit den für Testverfahren gängigen Quantilen in der Statistikliteratur vorliegen, lässt sich für ein vorgegebenes Testniveau, d.h. für eine Irrtumswahrscheinlichkeit $\alpha$ entscheiden, ob die Nullhypothese aufrecht erhalten werden kann oder abgelehnt werden muss.

Nicht unerwähnt bleiben soll jedoch der Fall des Vorliegens von Bindungen, falls es sich um diskretes Datenmaterial handelt. Da bei Bindungen gleiche Zahlenwerte mehrmals auftreten, lässt sich mit der Teststatistik $T_{m, n}$ in der hier vorliegenden Form nicht exakt rechnen. Vielmehr muss eine Korrektur der Testgröße vorgenommen werden, auf deren Darstellung hier wegen ihrer hohen Komplexität verzichtet werden soll. 395

Im Folgenden soll der weitere Verlauf der Durchführung des Testverfahrens beschrieben werden.

Die Nullhypothese wird abgelehnt, falls die normierte Teststatistik einen Wert liefert, der

- kleiner als $u_{\alpha / 2}$ bzw.

- größer als $u_{1-\alpha / 2}$ ist. 
Die beiden Größen $u_{\alpha / 2}$ und $u_{1-\alpha / 2}$ sind dabei das $\alpha / 2$ - Quantil, bzw. das (1 $\alpha / 2)$ - Quantil der Standardnormalverteilung.

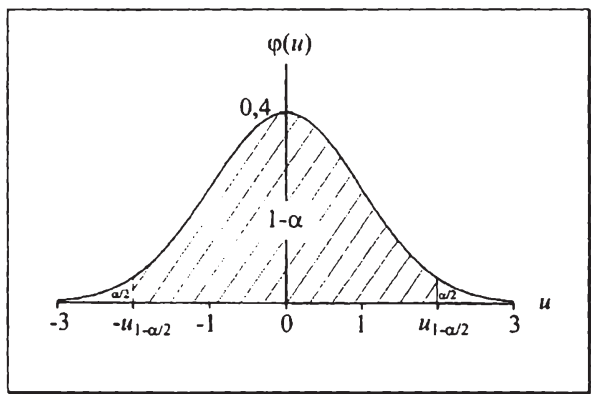

Abbildung 29: Das $\alpha / 2$ - und das (1 - $\alpha / 2)$ - Quantil der Standardnormalverteilung

Mit $\alpha$ wird der Fehler quantifiziert, der maximal möglich ist, die Nullhypothese fälschlicherweise abzulehnen.

Wird der Wilcoxon-Rangsummen-Test, wie im vorliegenden Fall, mit Hilfe der Statistik-Software SPSS durchgeführt, errechnet diese einen p-Value. Dieser Wert gibt an, bei welcher Fehlerwahrscheinlichkeit der Test zu einer Ablehnung der Nullhypothese führt. Je kleiner der $p$-Value, desto mehr spricht dies für die Ablehnung der Nullhypothese. Bei einer gängigen Irrtumswahrscheinlichkeit von $10 \%$ muss der $p$-Value demnach kleiner als 0,1 sein. In den meisten an späterer Stelle in dieser Studie betrachteten Fällen liegt er deutlich darunter.

\section{II.9.2 Kolmogorov-Smirnov-Anpassungstest}

Der im Folgenden beschriebene Kolmogorov-Smirnov-Anpassungstest ist wie der Wilcoxon-Rangsummen-Test ein nichtparametrisches Verfahren, so dass keine Verteilungsannahmen über das Datenmaterial vorausgesetzt werden. Das Testverfahren wird insbesondere bei stetigen Daten eingesetzt ${ }^{396}$, wie sie in den Hypothesen 2, 3, 4 und 5 vorliegen. In diesem Fall ist der KolmogorovSmirnov-Anpassungstest dem Wilcoxon-Rangsummen-Test vorzuziehen, da er mehr Informationen aus den Datensätzen einbezieht. In die Testgröße gehen 
dabei nicht nur die Ränge ein, sondern vielmehr werden auch die Größenordnungen der Variablen berücksichtigt.

Gegeben sind $z$ wei unabhängige Stichproben $X_{1}, X_{2}, \ldots, X_{m}$ und $Y_{1}, Y_{2}, \ldots, Y_{n}$, wobei der Gesamtumfang der Stichprobe $N=m+n$ entspricht.

Folgende Annahmen werden getroffen:397

1. $X_{1}, X_{2}, \ldots, X_{m}$ ist eine einfache Zufallsstichprobe, die einer Grundgesamtheit 1 (Population 1) entstammt. Die einzelnen Beobachtungen sind unabhängig und identisch verteilt.

$Y_{1}, Y_{2}, \ldots, Y_{n}$ ist eine einfache Zufallsstichprobe, die einer Grundgesamtheit 2 (Population 2) entstammt. Die einzelnen Beobachtungen sind unabhängig und identisch verteilt.

2. Die Beobachtungen $X_{1}, X_{2}, \ldots, X_{m}$ und $Y_{1}, Y_{2}, \ldots, Y_{n}$ sind gemeinsam voneinander unabhängig, d.h. es liegt Unabhängigkeit innerhalb und zwischen beiden Populationen vor.

3. Die Stichprobenvariablen $X_{1}, X_{2}, \ldots, X_{m}$ und $Y_{1}, Y_{2}, \ldots, Y_{n}$ haben stegige Verteilungsfunktionen $\mathrm{F}$ bzw. $\mathrm{G}$.

Die betrachtete Nullhypothese lautet: 398

$H_{0}: F(x)=G(x), \forall x \in \mathbb{R}$,

wobei $F(x)$ und $G(x)$ die stetigen Verteilungsfunktionen sind, die den Grundgesamtheiten zugrundeliegen.

Auch die Nullhypothese des Kolmogorov-Smirnov-Anpassungstests entspricht der des zuvor dargestellten Wilcoxon-Rangsummen-Tests. Der Unterschied zwischen beiden Verfahren liegt in der Teststatistik. Dabei werden zunächst zu beiden Stichproben die empirischen Verteilungsfunktionen $F_{m}(x)$ bzw. $G_{n}(x)$ gebildet: 399

397 Vgl. BÜNING/TRENKLER, 1994, S. 120.

$398 \mathrm{Vgl.} \mathrm{BÜNING/TRENKLER,} \mathrm{1994,} \mathrm{S.} 120$.

399 Vgl. PRATt/GiBBONS, 1981, S. $319 \mathrm{ff}$. 


$$
F_{m}(x)=\frac{1}{m} \sum_{i=1}^{m} 1_{\left\{x_{i} \leq x\right\}} \text { und } G_{n}(x)=\frac{1}{n} \sum_{j=1}^{n} 1_{\{y j \leq x\}}
$$

Dabei ist $1_{\{A\}}$ die Indikatorfunktion:

$$
1_{\{A\}}=\left\{\begin{array}{l}
1, \text { falls } A \text { wahr } \\
0, \text { sonst }
\end{array}\right.
$$

Der Kolmogorov-Smirnov-Anpassungstest baut auf folgender statistischen Grundaussage auf: Die empirischen Verteilungsfunktionen nähern sich mit wachsendem Stichprobenumfang immer mehr der wahren, aber unbekannten theoretischen Verteilungsfunktion. ${ }^{400}$ Die Testgröße berechnet den maximalen Abstand zwischen den beiden empirischen Verteilungsfunktionen, die sich aus den beiden Stichproben ergeben. Dafür verwendet man folgende Testgröße:

$$
D_{m, n}=\sup _{x \in R}\left|F_{m}(x)-G_{n}(x)\right|
$$

Ist dieser Wert zu groß, so spricht dies gegen die Gültigkeit der Nullhypothese.

Für die Verteilung der Testgröße sind die Quantile bekannt.

400 Vgl. zum Zentralsatz der Statistik: GIBBONS/CHAKRABORTI, 1992, S. 56. 


\section{Kapitel Ergebnisse der empirischen Untersuchung}

Nachdem im 9. Kapitel die statistischen Testverfahren ausgewählt und in der Theorie dargestellt wurden, stehen in diesem letzten Kapitel des Teils II die Anwendung der Verfahren, sowie die Darstellung der Untersuchungsergebnisse im Mittelpunkt. Die Interpretation der Ergebnisse wird anschließend im Teil III vorgenommen.

Die Darstellung erfolgt hypothesenweise und in enger Anlehnung an die Erläuterungen der vorherigen Kapitel, insbesondere an das 8. Kapitel, Abschnitt II.8.2, in dem die einzelnen Hypothesen hergeleitet werden.

Sowohl in den Hypothesen 1 und 6, die anhand des Wilcoxon-RangsummenTests untersucht werden, als auch in den Hypothesen 2 bis 5 , bei denen der Kolmogorov-Smirnov-Test Anwendung findet, wird ein ähnliches Vorgehen gewählt. Bei beiden Testverfahren und damit bei allen Hypothesen werden den XVariablen die Daten der Untersuchungsmenge und den Y-Variablen die der Kontrollmenge zugewiesen. Zwischen beiden Datenmengen bestehen, wie bereits erläutert, keinerlei Abhängigkeiten.

Alle sechs Nullhypothesen sind so formuliert, dass sie den Erwartungen zufolge in den Perioden 1 und 2 durch das jeweilige Testverfahren bestätigt werden soliten. Erst in den Folgeperioden, nachdem die Teams der Untersuchungsmenge Kenntnis über ihre günstige Kostenposition erhalten haben, sollten sich bei allen sechs Hypothesen Unterschiede zwischen den Teams der Untersuchungs- und der Kontrollmenge feststellen lassen. Diese Unterschiede sollten signifikant sein und $z u$ einer Ablehnung der jeweiligen Nullhypothese in den Perioden $3,4,5$ und 6 führen.

Als Signifikanzniveau wird allgemein eine Fehlerwahrscheinlichkeit von $10 \%$ gewählt. Dieser Wert kommt in der vorliegenden Erhebung in p-Values von 0,1 zum Ausdruck. Die Nullhypothese wird so mit einer maximalen Wahrscheinlichkeit von $10 \%$ fälschlicherweise abgelehnt. In diversen Untersuchungsreihen sinkt die Fehlerwahrscheinlichkeit in einzelnen Perioden, wie noch zu zeigen sein wird, auf ein Signifikanzniveau von $5 \%$ und teilweise sogar auf $1 \%$. Dies entspricht $p$-Values von 0,05 bzw. 0,01, die die jeweilige Hypothese stützen. 
Zur Beurteilung der Hypothesen wird nicht ausschließlich der absolute Wert des p-Values einzelner Perioden verwendet. Zusätzlich ist die zeitliche Entwicklung dieses Wertes über die Perioden hinweg zu betrachten. Für diese Studie sind die Veränderung von der Periode 2 zur Periode 3 und die weitere Entwicklung der $p$-Values nach der Periode 3 entscheidend, weil die Teams der Untersuchungsmenge nach der 2. Periode über ihre Kostenposition innerhalb ihrer Vierergruppe informiert werden und danach erstmals darauf reagieren können.

Je deutlicher der $p$-Value von Periode 2 auf Periode 3 sinkt, desto unterschiedlichere Entscheidungen werden von den Teams der Untersuchungs- und der Kontrollmenge getroffen. Selbst wenn der $p$-Value in der 3 . Periode nicht unter 0,1 fällt und damit das Signifikanzniveau von $10 \%$ überschritten wird, kann eine entsprechend deutliche und nachhaltige Veränderung des $p$-Values für die Beibehaltung der jeweiligen Annahme sprechen.

Im Folgenden werden die einzelnen Hypothesen besprochen.

\section{II.10.1 Hypothese 1: Strategisches Investitionsverhalten}

Zur Untersuchung des strategischen Investitionsverhaltens der Teams der Untersuchungs- und der Kontrollmenge ist folgende Nullhypothese $\mathrm{H}_{0} 1$ ) zu betrachten: Die Teams der Untersuchungs- und der Kontrollmenge, die sich alle in kostenführender Position befinden, zeigen in den einzelnen Perioden des Experiments kein signifikant unterschiedliches Investitionsverhalten, d.h. beide Teammengen verfügen über ähnlich viele Kapazitätseinheiten.

Aufgrund der diskreten Merkmalsausprägung des Datenmaterials ${ }^{401}$ findet der Wilcoxon-Rangsummen-Test Anwendung. Es werden die Kapazitätseinheiten des Maschinentyps erfasst, der für die Produktion des jeweiligen Produktes benötigt wird, bei dem das betrachtete Team die Kostenführung inne hat.

401 Vgl. HARTUNG/ELPELT, 1999, S. $18 \mathrm{f}$. 
Gemäß den Annahmen 1.a) und 1.b) wird in den Perioden 1 und 2 eine Bestätigung der Nullhypothese erwartet. Ab der 3. Periode sollte die Nullhypothese abzulehnen sein und ein signifikant stärkerer Kapazitätsaufbau bei den Teams der Untersuchungsmenge festzustellen sein.

Die Gültigkeit der Nullhypothese in den ersten beiden Perioden sollte durch pValues von größer als 0,1 bestätigt werden. In den Folgeperioden sind für eine Ablehnung der Nullhypothese dagegen p-Values kleiner als 0,1 notwendig. Der Wert leitet sich aus dem gewählten Signifikanzniveau von $10 \%$ ab.

Die Überprüfung der Hypothese wird insgesamt sechsmal durchgeführt, wobei exemplarisch das Produkt 1 beider Planspiele ausführlich erörtert wird. Die Anwendung des Wilcoxon-Rangsummen-Tests in SPSS ergibt für MARGA einen zweiteiligen Output, der in Tabelle 12 und Tabelle 13 dargestellt wird.

\begin{tabular}{|c|c|c|c|c|}
\hline & Zuordnung & $\mathbf{N}$ & $\begin{array}{l}\text { Mittlerer } \\
\text { Rang }\end{array}$ & $\begin{array}{l}\text { Rang- } \\
\text { summe }\end{array}$ \\
\hline \multirow[t]{3}{*}{ Periode 1} & Untersuchungsmenge & 36 & 35,40 & 1274,50 \\
\hline & Kontrollmenge & 35 & 36,61 & 1281,50 \\
\hline & Gesamt & 71 & & \\
\hline \multirow[t]{3}{*}{ Periode 2} & Untersuchungsmenge & 36 & 36,67 & 1320,00 \\
\hline & Kontrollmenge & 35 & 35,31 & 1236,00 \\
\hline & Gesamt & 71 & & \\
\hline \multirow[t]{3}{*}{ Periode 3} & Untersuchungsmenge & 36 & 40,36 & 1453,00 \\
\hline & Kontrollmenge & 35 & 31,51 & 1103,00 \\
\hline & Gesamt & 71 & & \\
\hline \multirow[t]{3}{*}{ Periode 4} & Untersuchungsmenge & 36 & 41,58 & 1497,00 \\
\hline & Kontrollmenge & 35 & 30,26 & 1059,00 \\
\hline & Gesamt & 71 & & \\
\hline \multirow[t]{3}{*}{ Periode 5} & Untersuchungsmenge & 36 & 40,49 & 1457,50 \\
\hline & Kontrollmenge & 35 & 31,39 & 1098,50 \\
\hline & Gesamt & 71 & & \\
\hline \multirow[t]{3}{*}{ Periode 6} & Untersuchungsmenge & 36 & 40,10 & 1443,50 \\
\hline & Kontrollmenge & 35 & 31,79 & 1112,50 \\
\hline & Gesamt & 71 & & \\
\hline
\end{tabular}

Tabelle 12: MARGA Hypothese 1, Produkt 1, Berechnung der Ränge 


\begin{tabular}{|l|c|c|c|c|c|c|}
\cline { 2 - 6 } \multicolumn{1}{c|}{} & Periode 1 & Periode 2 & Periode 3 & Periode 4 & Periode 5 & Periode 6 \\
\hline $\begin{array}{l}\text { p-Value, bzw. } \\
\text { asymptot. Signi- } \\
\text { fikanz (2-seitig) }\end{array}$ & 0,798 & 0,781 & 0,069 & 0,020 & 0,062 & 0,088 \\
\hline
\end{tabular}

Tabelle 13: MARGA Hypothese 1, Produkt 1, Teststatistik

Die Daten der 36 Teams der Untersuchungs- und die der 35 Teams der Kontrollmenge werden beim Wilcoxon-Rangsummen-Test der Größe nach aufsteigend geordnet. Den X-Werten, denen die Daten der Untersuchungsmenge zugewiesen sind, werden die entsprechenden Ränge der aufsteigenden Datenreihe zugeteilt, wobei an gleiche Werte mittlere Ränge vergeben werden. Danach bildet man die Summe aller Ränge der $X$-Werte und erhält die Rangsummen, die in Tabelle 12 ausgegeben werden. Der mittlere Rang ergibt sich aus der anschließenden Division der Rangsumme durch die Anzahl der Werte "N“. Bei gleichem Vorgehen erhält man die Werte der Kontrollmenge.

Der Tabelle 13 sind die p-Values zu entnehmen, die unter SPSS auch zweiseitige asymptotische Signifikanz genannt werden und anhand derer die Beurteilung über Annahme oder Ablehnung der Nullhypothese erfolgt. Mit 0,798 und 0,781 liegt dieser Wert eindeutig über der gesetzten Grenze von 0,1. Für die Perioden 1 und 2 wird die Nullhypothese damit erwartungsgemäß bestätigt. Ebenso klar kann aufgrund der ermittelten $p$-Values in den Periode 3, 4, 5 und 6, die deutlich kleiner als 0,1 sind und in der Periode 4 mit 0,02 ein Minimum erreichen, die Nullhypothese abgelehnt werden.

Damit unterstützen die Ergebnisse die Annahme 1.a), dass die Teams der Untersuchungs- und der Kontrollmenge in den Perioden 1 und 2 kein signifikant unterschiedliches Investitionsverhalten zeigen, sondem erst nachdem die Teams der Untersuchungsmenge über ihre Kostenvorteile informiert worden sind.

Die Annahme 1.b), nach der die Teams der Untersuchungsmenge nach der 2. Periode die Kapazitäten in stärkerem Maße ausbauen, als die der Kontrollmenge, lässt sich durch Tabelle 12 bekräftigen. Die Rangsumme und demnach auch der mittlere Rang, weisen in den Perioden 1 und 2 keine signifikanten Unterschiede auf. Erst in den Folgeperioden wird der starke Kapazitätsaufbau bei den Teams der Untersuchungsmenge sichtbar. Demnach kann auch die Annahme 1.b) auf dem vorgegebenen Signifikanzniveau aufrecht erhalten werden. 
Die bei MARGA am Beispiel des Produktes 1 gefundenen Ergebnisse sollten auch für das Planspiel THE BOARD zutreffen, was in Tabelle 14 und Tabelle 15 untersucht wird. Werte, die den Erwartungen nicht entsprechen, sind dort in fetter Schreibweise dargestellt.

Die für THE BOARD erhaltenen Ergebnisse für das Produkt 1 bestätigen die bei MARGA gefundenen Zusammenhänge nur tendenziell. In den Perioden 1 und 2 lässt sich wie bei MARGA die Nullhypothese bestätigen, da die p-Values deutlich größer als 0,1 sind. In den Folgeperioden sinken die Werte nicht erwartungsgemäß unter die gesetzte Fehlerwahrscheinlichkeit von 0,1. Die Nullhypothese kann für diese drei Perioden demnach nicht verworfen werden.

\begin{tabular}{|l|l|c|c|c|}
\cline { 2 - 5 } \multicolumn{1}{|c|}{} & Zuordnung & N & $\begin{array}{c}\text { Mittlerer } \\
\text { Rang }\end{array}$ & $\begin{array}{c}\text { Rang- } \\
\text { summe }\end{array}$ \\
\hline Periode 1 & Untersuchungsmenge & 59 & 55,71 & 3287,00 \\
\cline { 2 - 5 } & Kontrollmenge & 54 & 58,41 & 3154,00 \\
\cline { 2 - 5 } & Gesamt & 113 & & \\
\hline Periode 2 & Untersuchungsmenge & 59 & 55,18 & 3255,50 \\
\cline { 2 - 5 } & Kontrollmenge & 54 & 58,99 & 3185,50 \\
\cline { 2 - 5 } & Gesamt & 113 & & \\
\hline Periode 3 & Untersuchungsmenge & 59 & 60,81 & 3588,00 \\
\cline { 2 - 5 } & Kontrollmenge & 54 & 52,83 & 2853,00 \\
\cline { 2 - 5 } & Gesamt & 113 & & 3594,50 \\
\hline Periode 4 & Untersuchungsmenge & 59 & 60,92 & 2846,50 \\
\cline { 2 - 5 } & Kontrollmenge & 54 & 52,71 & 3553,50 \\
\cline { 2 - 5 } & Gesamt & 113 & & 2887,50 \\
\hline \multirow{4}{*}{ Periode 5 } & Untersuchungsmenge & 59 & 60,23 & \\
\cline { 2 - 5 } & Kontrollmenge & 54 & 53,47 & \\
\cline { 2 - 5 } & Gesamt & 113 & & \\
\hline
\end{tabular}

Tabelle 14: THE BOARD Hypothese 1, Produkt 1, Berechnung der Ränge

\begin{tabular}{|l|c|c|c|c|c|}
\cline { 2 - 6 } \multicolumn{1}{c|}{} & Periode 1 & Periode 2 & Periode 3 & Periode 4 & Periode 5 \\
\hline $\begin{array}{l}\text { p-Value, bzw. } \\
\text { asymptot. Signi- } \\
\text { fikanz (2-seitig) }\end{array}$ & 0,657 & 0,531 & $\mathbf{0 , 1 9 3}$ & $\mathbf{0 , 1 8 2}$ & $\mathbf{0 , 2 7 0}$ \\
\hline
\end{tabular}

Tabelle 15: THE BOARD Hypothese 1, Produkt 1, Teststatistik 
Trotz dieses unerwarteten Resultats lässt sich ein Trend feststellen, der der Entwicklung entspricht, die zuvor bei MARGA beobachtet werden konnte. Die pValues in den Perioden 1 und 2 sind um den Faktor drei größer als in den Perioden 3 und 4, so dass man durchaus von einer Veränderung der Investitionsstrategie der Teams der Untersuchungsmenge ausgehen kann - und auch ausgehen muss. Unterstützt wird diese Aussage durch Einbeziehung der Rangsumme bzw. des mittleren Ranges, wodurch zusätzlich auch die Annahme 1.b) zur Ausweitung der Produktionskapazitäten bei den Teams der Untersuchungsmenge nach der 2. Periode bekräftigt wird.

Auch der Anstieg des p-Values in der 5. Periode ist zu erklären. Wie bereits erläutert ${ }^{402}$, sind die jeweils letzten Perioden, bei MARGA die 6 . und bei THE BOARD die 5. Periode, oft - aber nicht immer - durch ein Verhalten geprägt, das stark durch das bevorstehende Ende des Planspiels beeinflusst ist. Bisher verfolgte Strategien werden dann zugunsten kurzfristiger Optimierungen aufgegeben. Als Beispiel sei hier ein Investitionsstop zum Ende des Planspiels angeführt, der aus taktischen Überlegungen heraus durchaus sinnvoll sein kann, wodurch aber die Aussagekraft der Daten der Hypothese 1 in der letzten Periode eingeschränkt ist. Aus diesem Grund sind in der vorliegenden Untersuchung die Daten der jeweils letzten Periode zwar Bestandteil der Auswertung, fließen indes nicht in die spätere Interpretation der Ergebnisse im Teil III ein.

In Tabelle 16 werden die p-Values aller sechs Versuchsreihen dargestellt, wobei wiederum unerwartete Werte fett hervorgehoben sind.

\begin{tabular}{|l|c|c|c|c|c|c|c|}
\cline { 2 - 8 } \multicolumn{2}{c|}{} & Periode 1 & Periode 2 & Periode 3 & Periode 4 & Periode 5 & Periode 6 \\
\hline & Prod. 1 & 0,798 & 0,781 & 0,069 & 0,020 & 0,062 & 0,088 \\
\cline { 2 - 8 } & Prod. 2 & 0,256 & 0,390 & 0,018 & 0,048 & 0,052 & 0,056 \\
\cline { 2 - 8 } & Prod. 3 & 0,823 & 0,743 & $\mathbf{0 , 2 6 3}$ & $\mathbf{0 , 2 6 1}$ & $\mathbf{0 , 2 8 6}$ & $\mathbf{0 , 2 9 4}$ \\
\hline \multirow{3}{*}{ THE BOARD } & Prod. 1 & 0,657 & 0,531 & $\mathbf{0 , 1 9 3}$ & $\mathbf{0 , 1 8 2}$ & $\mathbf{0 , 2 7 0}$ & -- \\
\cline { 2 - 8 } & Prod. 2 & 0,886 & 0,937 & $\mathbf{0 , 4 8 9}$ & $\mathbf{0 , 2 1 7}$ & $\mathbf{0 , 1 3 2}$ & - \\
\cline { 2 - 8 } & Prod. 3 & 0,762 & 0,863 & $\mathbf{0 , 2 7 6}$ & $\mathbf{0 , 3 3 2}$ & $\mathbf{0 , 3 1 2}$ & -- \\
\hline
\end{tabular}

Tabelle 16: Hypothese 1, p-Values 
Bei der Betrachtung der Werte wiederholt sich das zuvor bei THE BOARD beim Produkt 1 erläuterte Ergebnisbild: Auf Basis des Signifikanzniveaus von $10 \%$ führen vier Versuchsreihen nicht zu einer Ablehnung der Nullhypothese in den Perioden 3, 4, 5 und 6. Andererseits spricht die meist deutliche Veränderung der $p$-Values von Periode 2 auf die Folgeperioden für die Aufrechterhaltung der Annahme 1.a). Diese wird zusätzlich durch die Rangsummen bzw. die mittleren Ränge bekräftigt. 403

Zusammenfassend ist zu sagen, dass nach streng statistischem Vorgehen bei einem Signifikanzniveau von $10 \%$ die Nullhypothese nur in zwei von sechs Versuchsreihen erwartungsgemäß abgelehnt werden kann. Die Deutlichkeit, mit der sich eine Veränderung der $p$-Values von der 2. auf die Folgeperioden einstellt und die steigenden Rangsummen bzw. mittleren Ränge bei den Teams der Untersuchungsmenge, sprechen dennoch für eine Bekräftigung der Annahmen 1.a) und 1.b). Danach zeigen die Teams der Untersuchungsmenge nach Erhalt von Informationen über ihre Kostenposition dahingehend ein unterschiedliches Investitionsverhalten, dass sie ihre Kapazitäten stärker ausbauen, als die Teams der Kontrollmenge.

Obwohl die Untersuchungsergebnisse tendenziell den Erwartungen entsprechen, bleibt die Stärke der Ausprägung zu gering. Damit lässt sich die Hypothese 1 aufgrund der statistischen Auswertung des Datenmaterials auf einem Signifikanzniveau von $10 \%$ nicht aufrecht erhalten.

\section{II.10.2 Hypothese 2: Produktionsmengenpolitik}

Für die 2. Hypothese wurde folgende Nullhypothese $\mathrm{H}_{0} 2$ ) formuliert: Die Teams der Untersuchungs- und der Kontrollmenge weisen hinsichtlich der Produktionsmengenstrategie in den einzelnen Perioden der Untersuchung keine signifikanten Unterschiede auf, d.h. die prozentuale Abweichung der kumulierten Produktionsmenge des zu betrachtenden Kostenführers von der durchschnittlichen kumulierten Produktionsmenge aller vier Konkurrenten seiner Gruppe wird bei den Teams der Untersuchungs- und der Kontrollmenge ähnlich sein.

403 Auf eine detaillierte Darstellung der Rangsummen und der mittleren Ränge wird aus Platzgründen verzichtet. 
In den Perioden 1 und 2 wird gemäß den Annahmen 2.a) und 2.b) eine Bestätigung der Nullhypothese durch p-Values von größer als 0,1 erwartet. Erst nach der 2. Periode sollte es bei $p$-Values, die kleiner als 0,1 sind, zu einer Ablehnung der Nullhypothese kommen. Wiederum leitet sich dieser Wert aus der gesetzten Fehlerwahrscheinlichkeit von $10 \%$ ab. Kommt es nach der 2 . Periode zu der erwarteten Ablehnung der Nullhypothese, sollten die Teams der Untersuchungsmenge gemäß der Annahme 2.b) eine expansivere Mengenstrategie als die Teams der Kontrollmenge verfolgen. Übertragen auf das vorliegende Datenmaterial heißt das, dass nach der 2. Periode signifikant höhere prozentuale Abweichungen bei den Teams der Untersuchungsmenge festzustellen sein sollten.

Im Gegensatz zur 1. Hypothese weist das Datenmaterial hier eine stetige Merkmalsausprägung und ein metrisches Skalenniveau auf ${ }^{404}$, so dass der Kolmogorov-Smirnov-Test angewendet werden kann.

Die Hypothese 2 wird insgesamt sechsmal mit unterschiedlichen Datensätzen, d.h. für die drei Produkte beider Planspiele, durchgeführt. In Tabelle 17 werden die Ergebnisse in Anlehnung an den SPSS-Output beispielhaft für das Produkt 1 des Planspiels MARGA dargestellt:

\begin{tabular}{|l|l|c|c|c|c|c|c|}
\cline { 2 - 8 } \multicolumn{2}{c|}{} & Periode 1 & Periode 2 & Periode 3 & Periode 4 & Periode 5 & Periode 6 \\
\hline $\begin{array}{l}\text { Extremste } \\
\text { Differenzen }\end{array}$ & Absolut & 0,150 & 0,180 & 0,323 & 0,322 & 0,294 & 0,214 \\
\cline { 2 - 8 } & Positiv & 0,150 & 0,116 & 0,117 & 0,087 & 0,059 & 0,031 \\
\cline { 2 - 8 } & Negativ & 0,120 & 0,180 & 0,323 & 0,322 & 0,294 & 0,214 \\
\hline Kolmogorov-Smirnov-Z & 0,632 & 0,759 & 1,361 & 1,357 & 1,237 & 0,903 \\
\hline $\begin{array}{l}\text { p-Value, bzw. asymptot. } \\
\text { Signifikanz (2-seitig) }\end{array}$ & 0,819 & 0,612 & 0,049 & 0,050 & 0,094 & $\mathbf{0 , 3 8 9}$ \\
\hline
\end{tabular}

Tabelle 17: MARGA Hypothese 2, Produkt 1, Teststatistik

Mit der Kolmogorov-Smirnov-Teststatistik wird der Maximalabstand zwischen den empirischen Verteilungsfunktionen der $X$ - und der $Y$-Variablen ermittelt. Dieser Abstand ergibt sich aus dem Maximum der positiven und negativen Abstände, die für die einzelnen Perioden in Tabelle 17 aufgeführt sind. Liegen hinsichtlich der im Versuch erfassten Daten, also der prozentualen Abweichungen vom Durchschnitt, keine signifikanten Unterschiede vor, müssen sowohl positive als auch negative Abweichungen bzw. Differenzen vorhanden sein. 
Wie aus Tabelle 17 hervorgeht, liegen in den ersten beiden Perioden sowohl positive als auch negative Abweichungen vor. Die $p$-Values nehmen mit 0,819 und 0,612 Werte über 0,1 an, was erwartungsgemäß nicht zu einer Ablehnung der Nullhypothese $\mathrm{H}_{0} 2$ ) in den Perioden 1 und 2 führt.

In den Folgeperioden werden die positiven Differenzen im Verhältnis zu den negativen Differenzen immer kleiner. Dies ist als Indikator dafür zu sehen, dass die X-Variable die Y-Variable dominiert. ${ }^{405} \mathrm{Im}$ vorliegenden Fall ist dies so $\mathrm{zu}$ interpretieren, dass die X-Variable dazu tendiert, höhere Werte als die Y-Variable anzunehmen, die Teams der Untersuchungsmenge also eine expansivere Strategie wählen als die Teams der Kontrollmenge. Da die p-Values mit Ausnahme der 6. Periode unter 0,1 liegen, kann dieses Resultat auf einem Signifikanzniveau von $10 \%$ unterstützt werden. In Periode 6 weisen die Teams der Untersuchungs- und der Kontrollmenge keine signifikant unterschiedlichen Entscheidungen mehr auf, was auf das bevorstehende Ende des Planspiels und das daraus resultierende kurzfristig orientierte Taktieren der Teams zurückzuführen ist.

Die Annahmen 2.a) und 2.b) können für das gewählte Beispiel aufrechterhalten werden. Dies erhärtet sich bei Betrachtung der übrigen fünf Datensätze, deren vollständige SPSS-Outputs im Rahmen dieser Ausführungen nicht dargestellt werden sollen. In Tabelle 18 sind dafür die p-Values der sechs Versuchsreihen aufgeführt, die die Ergebnisse in konzentrierter Form abbilden. Dabei werden Werte, die den Erwartungen nicht entsprechen, wieder fett hervorgehoben.

\begin{tabular}{|l|c|c|c|c|c|c|c|}
\cline { 2 - 8 } \multicolumn{2}{c|}{} & Periode 1 & Periode 2 & Periode 3 & Periode 4 & Periode 5 & Periode 6 \\
\hline \multirow{3}{*}{ MARGA } & Prod. 1 & 0,819 & 0,612 & 0,049 & 0,050 & 0,094 & $\mathbf{0 , 3 8 9}$ \\
\cline { 2 - 8 } & Prod. 2 & 0,958 & 0,995 & 0,083 & 0,049 & 0,040 & 0,042 \\
\cline { 2 - 8 } & Prod. 3 & 0,892 & 0,417 & 0,094 & 0,095 & $\mathbf{0 , 1 6 8}$ & $\mathbf{0 , 2 7 0}$ \\
\hline THE BOARD & Prod. 1 & 0,926 & 0,780 & $\mathbf{0 , 1 0 3}$ & 0,034 & 0,031 & -- \\
\cline { 2 - 8 } & Prod. 2 & 0,540 & 0,890 & 0,079 & 0,084 & 0,054 & - \\
\cline { 2 - 8 } & Prod. 3 & 0,973 & 0,624 & $\mathbf{0 , 1 0 4}$ & 0,078 & 0,096 & - \\
\hline
\end{tabular}

Tabelle 18: Hypothese 2, p-Values

Durchgehend findet in den Perioden 1 und 2 die Nullhypothese die enwartete Bestätigung. Von Periode 2 auf Periode 3 wiederholt sich die bereits aus Hypo-

405 Dieser Zusammenhang wird stochastische Dominanz 1. Ordnung genannt. Vgl. LeHMANN, 1986, S. $65 \mathrm{ff}$. 
these 1 bekannte sprunghafte Reduzierung der p-Values, die auf eine Veränderung in der Produktionsmengenstrategie der Teams der Untersuchungsmenge nach der 2. Periode hinweist. In Periode 3 bewegen sich die $p$-Values teilweise eng um den gesetzten Grenzwert von 0,1 und liegen mit 0,103 und 0,104 nur knapp darüber. Bei strenger Vorgehensweise kann die Nullhypothese in diesen beiden Fällen auf dem gesetzten Signifikanzniveau von $10 \%$ daher nicht verworfen werden. Andererseits deutet die plötzliche Reduktion der Werte um den Faktor sechs bzw. sieben von Periode 2 auf Periode 3 eindeutig auf eine Veränderung in der Produktionsmengenstrategie bei den Teams der Untersuchungsmenge hin. Darüber hinaus spricht die Nachdrücklichkeit der Veränderung in den Folgeperioden dafür, die Annahme 2.a) weiterhin aufrecht zu erhalten. An diesem Gesamtbild, das sich im Rahmen der 2. Hypothese zeigt, ändern auch die $p$-Values bei MARGA, Produkt 3, von 0,168 und 0,270 in den Perioden 5 und 6 nichts, die wiederum unerwartet zu keiner Ablehnung der Nullhypothese in diesen Fällen führen. Hier wirkt sich das bereits erläuterte Verhalten der Teams gegen Ende des Planspiels aus, wovon jedoch die Annahme 2.a) in ihrer Grundaussage nicht betroffen sein sollte.

Die Annahme 2.b), nach der die Teams der Untersuchungsmenge eine expansivere Produktionsstrategie durchführen als die Teams der Kontrollmenge, wird durch die Analyse der extremsten Differenzen unterstützt.406 Das Vorgehen wurde zuvor am Beispiel des Produktes 1 bei MARGA gezeigt. Auch bei den restlichen Datensätzen unterstützen die Ergebnisse die Gültigkeit der Annahme 2.b).

Trotz einzelner $\mathrm{p}$-Values, die nicht innerhalb des erwarteten Bereichs liegen, spricht das Gesamtbild der Ergebnisse für eine Bekräftigung der Hypothese 2.

\section{II.10.3 Hypothese 3: Preispolitik}

In Hypothese 3 wird zur Untersuchung der Preispolitik folgende Nullhypothese $\mathrm{H}_{0} 3$ ) betrachtet: Die Teams der Untersuchungs- und der Kontrollmenge zeigen hinsichtlich ihrer Preisstrategie in den einzelnen Perioden keine signifikanten

406 Auf eine detaillierte Darstellung der extremsten Differenzen wird aus Platzgründen verzichtet. 
Unterschiede. Die prozentuale Abweichung des Preises des Kostenführers vom Durchschnittspreis aller vier Konkurrenten wird daher ähnlich sein.

Den Annahmen 3.a) und 3.b) folgend wird in den Perioden 1 und 2 die Nullhypothese durch $p$-Values von größer als 0,1 bestätigt. In den Folgeperioden sollte dagegen eine Ablehnung der Nullhypothese bei $p$-Values von kleiner als 0,1 erfolgen, da sich Unterschiede in der Preispolitik der Teams der Untersuchungs- und der Kontrollmenge zeigen. Diese Unterschiede sollten darin bestehen, dass die Teams der Untersuchungsmenge ein niedrigeres Preisniveau aufweisen als die Teams der Kontrollmenge.

Wiederum existieren zwischen den Daten der Untersuchungs- und der Kontrollmenge keinerlei Abhängigkeiten. Durch die Erfassung prozentualer Abweichungen vom Durchschnittspreis handelt es sich um ein stetiges Merkmal, so dass der Kolmogorov-Smirnov-Test angewendet werden kann. Darüber hinaus liegt ein metrisches Skalenniveau vor.

Die 3. Hypothese wird anhand von insgesamt 24 Datensätzen untersucht, d.h. drei Produkte auf jeweils vier Märkten und für beide Planspiele. Exemplarisch werden in Anlehnung an den SPSS-Output die Ergebnisse für das Produkt 1 auf dem Markt 1 des Planspiels MARGA in Tabelle 19 detailliert dargestellt und besprochen:

\begin{tabular}{|l|l|c|c|c|c|c|c|}
\cline { 2 - 8 } \multicolumn{2}{c|}{} & Periode 1 & Periode 2 & Periode 3 & Periode 4 & Periode 5 & Periode 6 \\
\hline $\begin{array}{l}\text { Extremste } \\
\text { Differenzen }\end{array}$ & Absolut & 0,114 & 0,143 & 0,266 & 0,297 & 0,290 & 0,360 \\
\cline { 2 - 8 } & Positiv & 0,100 & 0,124 & 0,266 & 0,297 & 0,290 & 0,360 \\
\cline { 2 - 8 } & Negativ & 0,114 & 0,143 & 0,000 & 0,000 & 0,000 & 0,000 \\
\hline \multicolumn{2}{|l}{ Kolmogorov-Smirnov-Z } & 0,481 & 0,602 & 1,120 & 1,250 & 1,224 & 1,515 \\
\hline $\begin{array}{l}\text { p-Value, bzw. asymptot. } \\
\text { Signifikanz (2-seitig) }\end{array}$ & 0,975 & 0,862 & $\mathbf{0 , 1 6 3}$ & 0,088 & 0,100 & 0,020 \\
\hline
\end{tabular}

Tabelle 19: MARGA Hypothese 3, Produkt 1, Markt 1, Teststatistik

Wie bei der 2. Hypothese wird auch hier bei der Kolmogorov-Smirnov-Teststatistik der Maximalabstand zwischen den empirischen Verteilungsfunktionen der $X$ - und der $Y$-Variablen ermittelt. In den ersten beiden Perioden treten dabei sowohl positive als auch negative Maximaldifferenzen auf, so dass bei korrespondierenden $p$-Values von 0,975 und 0,862 die Nullhypothese bestätigt wird. 
In den Folgeperioden nimmt die extremste negative Differenz den Wert Null an, während sich der extremste positive Wert erhöht. In diesen Fall dominiert die $Y$ Variable die X-Variable, d.h. die Kontrollmenge nimmt tendenziell höhere Werte an als die Untersuchungsmenge. Die prozentuale Abweichung des Preises des Kostenführers vom Durchschnittspreis aller vier Konkurrenten ist demnach bei den Teams der Untersuchungsmenge niedriger als bei den Teams der Kontrollmenge, was das erwartete niedrigere Preisniveau in der Untersuchungsmenge bestätigt. Der $p$-Value fällt von Periode 2 auf Periode 3 um mehr als das Fünffache auf 0,163 und liegt damit allerdings immer noch über der gesetzten Grenze von 0,1 , so dass für Periode 3 die Nullhypothese nicht, wie es in den nachfolgenden Perioden der Fall ist, widerlegt werden kann.

Trotz der auf dem 10\% Signifikanzniveau nicht widerlegbaren Nullhypothese in Periode 3 kann die Annahme 3.a) wegen des deutlichen Sprungs von Periode 2 auf Periode 3 und wegen der Nachdrücklichkeit in den Folgeperioden weiterhin aufrecht erhalten werden. Auch die Annahmen 3.b), wonach die Teams der Untersuchungsmenge ab der 3 . Periode ein niedrigeres Preisniveau als die Teams der Kontrollmenge wählen, wird durch die oben beschriebenen Ergebnisse in der beispielhaften Betrachtung eindrucksvoll unterstützt, da die negativen Maximaldifferenzen ab der 3. Periode sogar identisch null sind.

Tabelle 20 zeigt die ermittelten p-Values aller 24 Datensätze. Wie bei der exemplarischen Besprechung des Datensatzes MARGA, Produkt 1, Markt 1 lässt sich die Nullhypothese in den ersten beiden Perioden auch bei den übrigen Datensätzen ausnahmslos unterstützen. Erst in der 3. Periode treten vier Fälle auf, in denen die Nullhypothese nicht erwartungsgemäß abgelehnt werden kann, wobei hier - so wie zuvor - die Deutlichkeit und die Nachdrücklichkeit der Veränderung für die Gültigkeit der Annahme 3.a) sprechen. An dieser Aussage ändern auch die $p$-Values der Perioden 4 und 5 nichts, die nur knapp über dem Wert von 0,1 liegen, so dass die Nullhypothese hier nicht abgelehnt werden kann. Trotzdem liegen diese Werte noch in einem Rahmen, der die grundsätzliche Gültigkeit der Annahme 3.a) nicht in Frage stellt. Für den pValue von 0,249 in der 6. Periode gelten die bereits in beiden vorherigen Hypothesen erläuterten Zusammenhänge bezüglich des kurzfristigen Taktierens der Teams in der jeweils letzten Periode. 


\begin{tabular}{|c|c|c|c|c|c|c|c|c|}
\hline & & & Periode 1 & Periode 2 & Periode 3 & Periode 4 & Periode 5 & Periode 6 \\
\hline \multirow[t]{12}{*}{ MARGA } & \multirow[t]{4}{*}{ Prod. 1} & M 1 & 0,975 & 0,862 & 0,163 & 0,088 & 0,100 & 0,020 \\
\hline & & M 2 & 0,983 & 0,557 & 0,097 & 0,078 & 0,055 & 0,040 \\
\hline & & M3 & 0,862 & 0,819 & 0,094 & 0,102 & 0,010 & 0,010 \\
\hline & & M4 & 0,862 & 0,956 & 0,027 & 0,052 & 0,015 & 0,002 \\
\hline & \multirow[t]{4}{*}{ Prod. 2} & M1 & 0,992 & 0,925 & 0,180 & 0,029 & 0,088 & 0,249 \\
\hline & & M 2 & 0,991 & 0,757 & 0,142 & 0,005 & 0,024 & 0,085 \\
\hline & & M 3 & 0,928 & 0,975 & 0,091 & 0,011 & 0,044 & 0,085 \\
\hline & & M 4 & 0,646 & 0,993 & 0,100 & 0,083 & 0,023 & 0,078 \\
\hline & \multirow[t]{4}{*}{ Prod. 3} & M1 & 0,394 & 0,844 & 0,098 & 0,058 & 0,129 & 0,022 \\
\hline & & M 2 & 0,958 & 0,757 & 0,054 & 0,023 & 0,131 & 0,079 \\
\hline & & M 3 & 0,629 & 0,925 & 0,026 & 0,043 & 0,039 & 0,011 \\
\hline & & M 4 & 0,618 & 0,948 & 0,057 & 0,074 & 0,073 & 0,082 \\
\hline \multirow[t]{12}{*}{ THE BOARD } & \multirow{4}{*}{ Prod. 1} & M 1 & 0,384 & 0,494 & 0,017 & 0,061 & 0,018 & -- \\
\hline & & M 2 & 0,641 & 0,864 & 0,077 & 0,066 & 0,079 & - \\
\hline & & M 3 & 0,960 & 0,667 & 0,053 & 0,109 & 0,075 & -- \\
\hline & & M 4 & 0,763 & 0,596 & 0,060 & 0,069 & 0,095 & - \\
\hline & \multirow[t]{4}{*}{ Prod. 2} & M 1 & 0,748 & 0,940 & 0,091 & 0,077 & 0,054 & -- \\
\hline & & M 2 & 0,537 & 0,931 & 0,075 & 0,033 & 0,030 & -- \\
\hline & & M3 & 0,905 & 0,905 & 0,098 & 0,060 & 0,036 & -- \\
\hline & & M 4 & 0,730 & 0,597 & 0,125 & 0,058 & 0,073 & -- \\
\hline & \multirow[t]{4}{*}{ Prod. 3} & M 1 & 0,988 & 0,835 & 0,096 & 0,078 & 0,090 & - \\
\hline & & M 2 & 0,193 & 0,628 & 0,076 & 0,013 & 0,020 & - \\
\hline & & M 3 & 0,989 & 0,626 & 0,090 & 0,044 & 0,051 & -- \\
\hline & & M 4 & 0,731 & 0,882 & 0,048 & 0,055 & 0,060 & - \\
\hline
\end{tabular}

Tabelle 20: Hypothese 3, p-Values

Die Betrachtung der Annahme 3.b) basiert auf der bereits erläuterten Analyse der maximalen Differenzen, die aus Platzgründen hier nicht für alle Datensätze detailliert erörtert werden soll. Demnach tendieren nach der 2. Periode die negativen Differenzen gegen Null, während sich die positiven Differenzen erhöhen. Diese Beobachtungen sind wie im zuvor erörterten Beispiel so zu deuten, dass die Teams der Untersuchungsmenge erwartungsgemäß zu einem niedrigeren Preisniveau anbieten als die Teams der Kontrollmenge. Die Ergebnisse unterstützen damit auch die Annahme 3.b).

Zusammenfassend kann die Hypothese 3 auf einem Signifikanzniveau von 10\% weiterhin aufrecht erhalten werden. 


\section{II.10.4 Hypothese 4: Forschungs- und Entwicklungspolitik}

Die Produktqualität wird bei MARGA über das Forschungs- und Entwicklungsbudget gesteuert. Im Mittelpunkt der 4. Hypothese steht die von den Teams durchgeführte Strategie im F\&E-Bereich. Zur Untersuchung der Zusammenhänge wird folgende Nullhypothese $\mathrm{H}_{0} 4$ ) betrachtet: Die Teams der Untersuchungs- und der Kontrollmenge weisen in den einzelnen Perioden der Untersuchung keine signifikanten Unterschiede bezüglich ihrer F\&E-Strategie auf. Die prozentuale Abweichung des kumulierten F\&E-Budgets des Kostenführers vom durchschnittlichen kumulierten F\&E-Budget aller Konkurrenten wird daher in der Untersuchungs- und in der Kontrollmenge ähnlich sein.

Durch die Erfassung prozentualer Abweichungen sind die Daten stetig, so dass der Kolmogorov-Smirnov-Test anzuwenden ist. Sie weisen darüber hinaus ein metrisches Skalenniveau auf.

Den Annahmen 4.a) und 4.b) zufolge wird in den Perioden 1 und 2 eine Bestätigung der Nullhypothese bei $p$-Values von größer als 0,1 erwartet. Erst in den Folgeperioden, nachdem die Teams der Untersuchungsmenge Informationen über ihre Kostenposition erhalten haben, sollten diese ein erhöhtes F\&EBudget im Rahmen ihrer expansiveren Marketing-Strategie aufweisen. Für die Ausprägung der Daten bedeutet dies, dass nach der 2. Periode bei $p$-Values von kleiner als 0,1 signifikant höhere prozentuale Abweichungen im F\&EBudget bei den Teams der Untersuchungsmenge festzustellen sein sollten als bei den Teams der Kontrollmenge.

Die Hypothese 4 wird insgesamt sechsmal mit unterschiedlichen Datensätzen überprüft, nämlich für die drei Produkte beider Planspiele. In Tabelle 21 werden die Ergebnisse in Anlehnung an den SPSS-Output beispielhaft für das Produkt 1 des Planspiels MARGA dargestellt.

Die Ermittlung der Maximaldifferenzen zwischen den empirischen Verteilungsfunktionen der $X$ - und der $Y$-Variable beim Kolmogorov-Smirnov-Test ergeben für die ersten beiden Perioden positive und negative Werte, wie der Tabelle 21 zu entnehmen ist. Bei $p$-Values von 0,757 und 0,557 bestätigt sich damit die Nullhypothese. 


\begin{tabular}{|l|l|c|c|c|c|c|c|}
\cline { 2 - 8 } \multicolumn{2}{c|}{} & Periode 1 & Periode 2 & Periode 3 & Periode 4 & Periode 5 & Periode 6 \\
\hline $\begin{array}{l}\text { Extremste } \\
\text { Differenzen }\end{array}$ & Absolut & 0,160 & 0,188 & 0,294 & 0,356 & 0,323 & 0,348 \\
\cline { 2 - 8 } & Positiv & 0,160 & 0,188 & 0,000 & 0,000 & 0,000 & 0,000 \\
\cline { 2 - 8 } & Negativ & 0,110 & 0,087 & 0,294 & 0,356 & 0,323 & 0,348 \\
\hline \multicolumn{2}{|l|}{ Kolmogorov-Smirnov-Z } & 0,672 & 0,792 & 1,237 & 1,498 & 1,361 & 1,468 \\
\hline $\begin{array}{l}\text { p-Value, bzw. asymptot. } \\
\text { Signifikanz (2-seitig) }\end{array}$ & 0,757 & 0,557 & 0,094 & 0,023 & 0,049 & 0,027 \\
\hline
\end{tabular}

Tabelle 21: MARGA Hypothese 4, Produkt 1, Teststatistik

In den Folgeperioden nehmen die positiven Differenzen den Wert Null an, während sich die negativen Differenzen im Wert erhöhen. Dies zeigt wie in Hypothese 2 eine Dominanz der $\mathrm{X}$-Variablen gegenüber der $\mathrm{Y}$-Variablen an, was im vorliegenden Fall so zu verstehen ist, dass die X-Variable dazu tendiert, höhere Werte als die Y-Variable anzunehmen. Die Teams der Untersuchungsmenge setzen also relativ zu ihren Konkurrenten höhere F\&E-Budgets ein als die Teams der Kontrollmenge zu deren Wettbewerbern. Dieses Ergebnis kann für das vorliegende Beispiel auf einem Signifikanzniveau von $10 \%$, in den Perioden 4, 5 und 6 sogar von $5 \%$, bestätigt werden, da die p-Values dieser Perioden unter 0,1 bzw. 0,05 liegen. Damit können beide Annahmen 4.a) und 4.b) für das gewählte Beispiel, MARGA, Produkt 1, aufrecht erhalten werden.

Da sämtliche Ergebnisse der übrigen fünf Datensätze der 4. Hypothese aus Platzgründen nicht vollständig dargestellt und besprochen werden sollen, zeigt Tabelle 22 nur die p-Values aller sechs Datensätze:

\begin{tabular}{|l|c|c|c|c|c|c|c|}
\cline { 2 - 8 } \multicolumn{2}{c|}{} & Periode 1 & Periode 2 & Periode 3 & Periode 4 & Periode 5 & Periode 6 \\
\hline \multirow{4}{*}{ MARGA } & Prod. 1 & 0,757 & 0,557 & 0,094 & 0,023 & 0,049 & 0,027 \\
\cline { 2 - 8 } & Prod. 2 & 0,946 & 0,789 & 0,098 & 0,043 & 0,010 & 0,021 \\
\cline { 2 - 8 } & Prod. 3 & 0,937 & 0,799 & 0,089 & 0,095 & 0,097 & 0,168 \\
\hline THE BOARD & Prod. 1 & 0,288 & 0,507 & 0,091 & 0,055 & 0,087 & - \\
\cline { 2 - 8 } & Prod. 2 & 0,888 & 0,984 & 0,085 & 0,053 & 0,034 & - \\
\cline { 2 - 8 } & Prod. 3 & 0,882 & 0,780 & 0,078 & 0,042 & 0,065 & - \\
\hline
\end{tabular}

Tabelle 22: Hypothese 4, p-Values

Es ist zu sehen, dass sich die p-Values aller sechs Datensätze - mit einer Ausnahme - im enwarteten Rahmen der jeweiligen Periode bewegen. Hinzu kommen die sprunghafte Reduktion der Werte von Periode 2 auf Periode 3 sowie 
die Nachhaltigkeit dieser Tendenz in den Folgeperioden. Die genannte Ausnahme betrifft den Datensatz MARGA, Produkt 3, mit einem p-Value von 0,168 in der 6. Periode. Da sich hier das bereits erläuterte Verhalten der Teams vor dem Ende des Planspiels auswirkt, wird diesem Wert wie in den vorherigen Hypothesen nur eine begrenzte Aussagefähigkeit zugesprochen, so dass die Annahme 4.a) für alle sechs Datensätze aufrechterhalten werden kann.

Die Annahme 4.b), nach der die Teams der Untersuchungsmenge nach der 2. Periode relativ zu den Teams der Kontrollmenge ein erhöhtes F\&E-Budget im Rahmen ihrer expansiveren Politik aufweisen, wird durch die Analyse der extremsten Differenzen betrachtet. Die Vorgehensweise entspricht dem oben beschriebenen Beispiel, wobei im Rahmen dieser Ausführungen darauf verzichtet werden soll, die Untersuchung für alle Datensätze detailliert darzustellen, zumal die Daten ebenfalls die Annahme 4.b) unterstützen.

Somit können beide Annahmen 4.a) und 4.b) auf Basis des vorliegenden Datenmaterials und auf einem Signifikanzniveau von $10 \%$ aufrecht erhalten werden. Die Hypothese 4 wird damit weiterhin gestützt.

\section{II.10.5 Hypothese 5: Werbestrategie}

In Hypothese 5 wird der Einsatz der Werbung untersucht. Dabei wird folgende Nullhypothese $\mathrm{H}_{0} 5$ ) betrachtet: Die Teams der Untersuchungs- und der Kontrollmenge weisen in den einzelnen Perioden keine signifikanten Unterschiede bezüglich ihrer Werbeaufwendungen auf. Die prozentuale Abweichung des kumulierten Werbebudgets des Kostenführers vom durchschnittlichen kumulierten Werbebudget aller Konkurrenten wird damit in der Untersuchungs- und in der Kontrollmenge ähnlich sein.

Das Datenmaterial ist stetig und weist ein metrisches Skalenniveau auf, so dass der Kolmogorov-Smirnov-Test Anwendung findet.

Gemäß den Annahmen 5.a) und 5.b) wird in den ersten beiden Perioden eine Bestätigung der Nullhypothese bei p-Values von größer als 0,1 erwartet. Erst nachdem die Teams der Untersuchungsmenge nach der 2. Periode Informationen über die Kostenposition innerhalb ihrer Gruppe erhalten haben, sollten 
sich signifikante Unterschiede in der Werbestrategie zwischen der Untersuchungs- und der Kontrollmenge durch p-Values, die kleiner als 0,1 sind, zeigen. Dabei wird angenommen, dass die Teams der Untersuchungsmenge im Zuge einer aggressiven Marketing-Strategie relativ zu ihren Wettbewerbern ein höheres Werbebudget einsetzen, als die Teams der Kontrollmenge.

Die 5. Hypothese wird anhand von insgesamt 24 Datensätzen untersucht, d.h. drei Produkte auf jeweils vier Märkten und für beide Planspiele. Exemplarisch werden in Anlehnung an den SPSS-Output die Ergebnisse für das Produkt 1 auf dem Markt 1 des Planspiels MARGA in Tabelle 23 dargestellt und besprochen:

\begin{tabular}{|l|l|c|c|c|c|c|c|}
\cline { 2 - 8 } \multicolumn{2}{c|}{} & Periode 1 & Periode 2 & Periode 3 & Periode 4 & Periode 5 & Periode 6 \\
\hline $\begin{array}{l}\text { Extremste } \\
\text { Differenzen }\end{array}$ & Absolut & 0,125 & 0,210 & 0,294 & 0,300 & 0,326 & 0,268 \\
\cline { 2 - 8 } & Positiv & 0,121 & 0,144 & 0,058 & 0,029 & 0,052 & 0,031 \\
\cline { 2 - 8 } & Negativ & 0,125 & 0,210 & 0,294 & 0,300 & 0,326 & 0,268 \\
\hline \multicolumn{2}{|l|}{ Kolmogorov-Smirnov-Z } & 0,528 & 0,886 & 1,240 & 1,264 & 1,374 & 1,130 \\
\hline $\begin{array}{l}\text { p-Value, bzw. asymptot. } \\
\text { Signifikanz (2-seitig) }\end{array}$ & 0,943 & 0,412 & 0,092 & 0,082 & 0,046 & $\mathbf{0 , 1 5 5}$ \\
\hline
\end{tabular}

Tabelle 23: MARGA Hypothese 5, Produkt 1, Markt 1, Teststatistik

Die beim Kolmogorov-Smirnov-Testverfahren ermittelten positiven und negativen Extremdifferenzen treten in den Perioden 1 und 2 bei dazugehörigen $p$ Values von 0,943 und 0,412 auf, so dass die Nullhypothese für beide Perioden bestätigt werden kann.

In den Folgeperioden neigen die positiven Differenzen dazu, deutlich kleinere Werte anzunehmen, während sich die negativen Differenzen im Wert erhöhen. Damit wird die Y-Variable von der X-Variablen dominiert. Übertragen auf die in der 5. Hypothese zu untersuchende Problematik heißt dies, dass die Teams der Untersuchungsmenge ab Periode 3 relativ zu ihren Wettbewerbern höhere Werbebudgets einsetzen als die Teams der Kontrollmenge. Gestützt wird diese Aussage durch $p$-Values, die unterhalb der gesetzten Grenze von 0,1 liegen, so dass die Nullhypothese enwartungsgemäß verworfen werden kann. Der in der 6. Periode ermittelte p-Value von 0,155 wird auf die bereits beschriebenen kurzfristig orientierten Entscheidungen der Teams in der letzten Periode zurückgeführt. Daher ist die Aussagekraft dieses Wertes nur als gering zu bewerten. 
Damit führen diese Ergebnisse für den gewählten Datensatz MARGA, Produkt 1, Markt 1, auf einem Signifikanzniveau von $10 \%$ zu einer Unterstützung der Annahmen 5.a) und 5.b).

Die p-Values aller 24 Datensätze werden in Tabelle 24 dargestellt, wobei unerwartet über 0,1 liegende Werte wieder hervorgehoben sind:

\begin{tabular}{|c|c|c|c|c|c|c|c|c|}
\hline & & & Periode 1 & Periode 2 & Periode 3 & Periode 4 & Periode 5 & Periode 6 \\
\hline \multirow[t]{12}{*}{ MARGA } & \multirow[t]{4}{*}{ Prod. 1} & M1 & 0,943 & 0,412 & 0,092 & 0,082 & 0,046 & 0,155 \\
\hline & & M 2 & 0,937 & 0,451 & 0,105 & 0,085 & 0,046 & 0,083 \\
\hline & & M 3 & 0,394 & 0,403 & 0,091 & 0,023 & 0,082 & 0,051 \\
\hline & & M 4 & 0,451 & 0,456 & 0,110 & 0,091 & 0,046 & 0,109 \\
\hline & \multirow[t]{4}{*}{ Prod. 2} & M 1 & 0,887 & 0,623 & 0,091 & 0,044 & 0,041 & 0,040 \\
\hline & & M 2 & 0,623 & 0,967 & 0,092 & 0,053 & 0,022 & 0,005 \\
\hline & & M 3 & 0,892 & 0,892 & 0,077 & 0,042 & 0,077 & 0,079 \\
\hline & & M4 & 0,371 & 0,963 & 0,095 & 0,022 & 0,081 & 0,180 \\
\hline & \multirow[t]{4}{*}{ Prod. 3} & M1 & 0,646 & 0,773 & 0,050 & 0,081 & 0,000 & 0,079 \\
\hline & & M2 & 1,000 & 0,993 & 0,097 & 0,092 & 0,053 & 0,023 \\
\hline & & M 3 & 0,986 & 0,925 & 0,050 & 0,046 & 0,022 & 0,025 \\
\hline & & M4 & 0,862 & 0,437 & 0,054 & 0,012 & 0,058 & 0,024 \\
\hline \multirow[t]{12}{*}{ THE BOARD } & \multirow[t]{4}{*}{ Prod. 1} & M1 & 0,470 & 0,574 & 0,048 & 0,089 & 0,056 & - \\
\hline & & \begin{tabular}{|l|} 
M 2 \\
\end{tabular} & 0,644 & 0,969 & 0,088 & 0,055 & 0,029 & - \\
\hline & & M 3 & 0,969 & 0,855 & 0,060 & 0,035 & 0,035 & - \\
\hline & & M 4 & 0,937 & 0,912 & 0,101 & 0,063 & 0,011 & - \\
\hline & \multirow[t]{4}{*}{ Prod. 2} & M1 & 0,874 & 0,876 & 0,077 & 0,010 & 0,034 & - \\
\hline & & M 2 & 0,882 & 0,406 & 0,026 & 0,019 & 0,031 & - \\
\hline & & M 3 & 0,465 & 0,774 & 0,098 & 0,036 & 0,098 & - \\
\hline & & M4 & 0,990 & 0,939 & 0,122 & 0,031 & 0,030 & - \\
\hline & \multirow[t]{4}{*}{ Prod. 3} & M1 & 0,998 & 0,603 & 0,097 & 0,066 & 0,113 & - \\
\hline & & \begin{tabular}{|l|} 
M 2 \\
\end{tabular} & 0,505 & 0,664 & 0,051 & 0,052 & 0,044 & - \\
\hline & & $\begin{array}{ll}\text { M } 3 \\
\end{array}$ & 1,000 & 0,997 & 0,063 & 0,024 & 0,060 & - \\
\hline & & M 4 & 0,726 & 0,878 & 0,067 & 0,076 & 0,035 & - \\
\hline
\end{tabular}

Tabelle 24: Hypothese 5, p-Values

Die $p$-Values der beiden ersten Perioden liegen ausnahmslos innerhalb der Erwartungen, d.h. über dem Wert von 0,1 , so dass die Nullhypothese unterstützt wird. Ab Periode 3 sinken die p-Values deutlich und nachhaltig von wenigen Ausnahmen abgesehen auf unter 0,1, was zu der enwartungsgemäßen Ablehnung der Nullhypothese auf dem Signifikanzniveau von $10 \%$ führt. Die Ausnahmen betreffen Datensätze, in denen für einzelne Perioden die Null- 
hypothese nach strenger Vorgehensweise nicht verworfen werden kann, weil die $p$-Values über dem Wert von 0,1 liegen. Die vier auffälligen Werte in Periode 3 sinken von Periode 2 auf Periode 3 sprunghaft um ein Vielfaches, liegen nur knapp über dem Wert von 0,1 und sinken in den Folgeperioden weiter unter den Wert von 0,1. Die übrigen vier Fälle betreffen die jeweils letzte Periode des Planspiels, auf deren eingeschränkte Aussagekraft bereits mehrfach hingewiesen wurde. Die Annahme 5.a), der zufolge sich nach der 2. Periode signifikante Unterschiede in der Werbestrategie zwischen der Untersuchungs- und der Kontrollmenge zeigen, kann damit weiterhin aufrecht erhalten werden.

Die Annahme 5.b), nach der die Teams der Untersuchungsmenge relativ zu ihren Wettbewerbern ein höheres Werbebudget einsetzen als die Teams der Kontrollmenge, wird durch die Analyse der extremsten Differenzen untersucht. Das Vorgehen wurde bereits für das MARGA-Produkt 1 auf dem Markt 1 exemplarisch dargestellt, so dass hier aus Platzgründen auf eine detaillierte Besprechung aller 24 Datensätze verzichtet werden kann. Wie im oben beschriebenen Beispiel tendieren nach der 2. Periode die positiven Maximaldifferenzen gegen Null, während sich die negativen Differenzen im Wert erhöhen, was so zu interpretieren ist, dass die Teams der Untersuchungsmenge relativ zu ihren Wettbewerbern höhere Werbebudgets einsetzen als die Teams der Kontrollmenge. Dieses Ergebnis unterstützt die Annahme 5.b).

Zusammenfassend ist festzuhalten, dass die Hypothese 5 auf der Basis des vorliegenden Datenmaterials und auf einem Signifikanzniveau von $10 \%$ weiterhin aufrecht erhalten werden kann.

\section{II.10.6 Hypothese 6: Wirtschaftlicher Erfolg}

Während die Hypothesen 1 bis 5 bestimmte strategische Entscheidungen der Planspiel-Teams fokussieren, steht in der 6. Periode der erzielte Erfolg, gemessen an der Platzierung, im Mittelpunkt. Folgende Nullhypothese $\mathrm{H}_{0} 6$ ) ist in diesem Zusammenhang zu betrachten: Die Teams der Untersuchungs- und der Kontrollmenge weisen in den einzelnen Perioden keine signifikanten Unterschiede bezüglich ihrer Platzierung auf. 
Zwischen den Datenmengen bestehen keinerlei Abhängigkeiten. Es liegt ein diskreter Merkmalstyp vor, so dass der Wilcoxon-Rangsummen-Test anzuwenden ist.

Gemäß den Annahmen 6.a) und 6.b) wird in den Perioden 1 und 2 eine Bestätigung der Nullhypothese bei p-Values von größer als 0,1 erwartet. Erst in den Folgeperioden sollte die Nullhypothese auf der Basis von $p$-Values von kleiner als 0,1 abzulehnen sein, weil sich ein Unterschied zwischen den Teams der Untersuchungs- und der Kontrollmenge zeigt. Dieser sollte darin bestehen - so die Annahme 6.b) - dass die Teams der Untersuchungsmenge eine höhere Platzierung aufweisen.

Die Überprüfung der Nullhypothese kann mit dem vorhandenen Datenmaterial insgesamt sechsmal durchgeführt werden, für beide Planspiele mit jeweils drei Produkten. In Tabelle 25 und Tabelle 26 wird exemplarisch der zweiteilige SPSS-Output für das Produkt 1 des Planspiels MARGA bei Anwendung des Wilcoxon-Rangsummen-Tests dargestellt:

\begin{tabular}{|c|c|c|c|c|}
\hline & Zuordnung & $\mathbf{N}$ & $\begin{array}{c}\text { mittlerer } \\
\text { Rang }\end{array}$ & $\begin{array}{l}\text { Rang- } \\
\text { summe }\end{array}$ \\
\hline \multirow[t]{3}{*}{ Periode 1} & Untersuchungsmenge & 36 & 35,93 & 1293,50 \\
\hline & Kontrollmenge & 35 & 36,07 & 1262,50 \\
\hline & Gesamt & 71 & & \\
\hline \multirow[t]{3}{*}{ Periode 2} & Untersuchungsmenge & 36 & 37,24 & 1340,50 \\
\hline & Kontrollmenge & 35 & 34,73 & 1215,50 \\
\hline & Gesamt & 71 & & \\
\hline \multirow[t]{3}{*}{ Periode 3} & Untersuchungsmenge & 36 & 31,74 & 1142,50 \\
\hline & Kontrollmenge & 35 & 40,39 & 1413,50 \\
\hline & Gesamt & 71 & & \\
\hline \multirow[t]{3}{*}{ Periode 4} & Untersuchungsmenge & 36 & 31,08 & 1119,00 \\
\hline & Kontrollmenge & 35 & 41,06 & 1437,00 \\
\hline & Gesamt & 71 & & \\
\hline \multirow[t]{3}{*}{ Periode 5} & Untersuchungsmenge & 36 & 29,69 & 1069,00 \\
\hline & Kontrollmenge & 35 & 42,49 & 1487,00 \\
\hline & Gesamt & 71 & & \\
\hline \multirow[t]{3}{*}{ Periode 6} & Untersuchungsmenge & 36 & 31,75 & 1143,00 \\
\hline & Kontrollmenge & 35 & 40,37 & 1413,00 \\
\hline & Gesamt & 71 & & \\
\hline
\end{tabular}

Tabelle 25: MARGA Hypothese 6, Produkt 1, Berechnung der Ränge 


\begin{tabular}{|l|c|c|c|c|c|c|}
\cline { 2 - 6 } \multicolumn{1}{c|}{} & Periode 1 & Periode 2 & Periode 3 & Periode 4 & Periode 5 & Periode 6 \\
\hline $\begin{array}{l}\text { p-Value, bzw. } \\
\text { asymptot. Signi- } \\
\text { fikanz (2-seitig) }\end{array}$ & 0,975 & 0,595 & 0,068 & 0,035 & 0,007 & 0,062 \\
\hline
\end{tabular}

Tabelle 26: MARGA Hypothese 6, Produkt 1, Teststatistik

Wie in Tabelle $26 \mathrm{zu}$ sehen ist, nehmen die p-Values in den ersten beiden Perioden Werte an, die über 0,1 liegen, so dass die Nullhypothese weiterhin aufrecht zu erhalten ist. Ab Periode 3 sinken die $p$-Values ebenfalls sehr deutlich unter den Wert von 0,1. Damit kann die Nullhypothese auf einem Signifikanzniveau von $10 \%$ und weniger verworfen werden.

Dieses Ergebnis unterstützt die Annahme 6.a), nach der die Teams der Untersuchungs- und der Kontrollmenge in den Perioden 1 und 2 keine signifikanten Unterschiede bezüglich ihrer Platzierung aufweisen, sondern Unterschiede erst nach der 2. Periode, nachdem die Teams der Untersuchungsmenge Informationen über ihre Kostenposition erhalten haben, feststellbar sind.

Bei Betrachtung der Rangsummen bzw. der mittleren Ränge in Tabelle 25, kann die Annahme 6.b) analysiert werden, nach der für die Teams der Untersuchungsmenge nach der 2. Periode höhere Platzierungen erwartet werden. Demnach steigen die Werte in der Kontrollmenge nach der 2. Periode deutlich an, während sie in der Untersuchungsmenge sinken. Da die höchste Platzierung - im Sinne von „beste“ Platzierung - den Wert 1 und die niedrigste Platzierung den Wert 4 erhält, erreichen die Teams der Untersuchungsmenge höhere Platzierungen und schneiden daher erfolgreicher ab als die Teams der Kontrollmenge. Auch die Annahme 6.b) kann damit aufgrund der vorliegenden Ergebnisse des Datensatzes MARGA, Produkt 1, aufrecht erhalten werden.

\begin{tabular}{|l|c|c|c|c|c|c|c|}
\cline { 2 - 8 } \multicolumn{2}{c|}{} & Periode 1 & Periode 2 & Periode 3 & Periode 4 & Periode 5 & Periode 6 \\
\hline \multirow{3}{*}{ MARGA } & Prod. 1 & 0,975 & 0,595 & 0,068 & 0,035 & 0,007 & 0,062 \\
\cline { 2 - 8 } & Prod. 2 & 0,681 & 0,834 & 0,074 & 0,028 & 0,026 & 0,024 \\
\cline { 2 - 8 } & Prod. 3 & 0,639 & 0,819 & $\mathbf{0 , 1 6 6}$ & 0,038 & 0,038 & 0,031 \\
\hline \multirow{3}{*}{ THE BOARD } & Prod. 1 & 0,669 & 0,494 & $\mathbf{0 , 1 0 7}$ & 0,014 & 0,020 & - \\
\cline { 2 - 8 } & Prod. 2 & 0,693 & 0,901 & $\mathbf{0 , 1 3 9}$ & 0,021 & 0,012 & - \\
\cline { 2 - 8 } & Prod. 3 & 0,416 & $\mathbf{0 , 8 1 0}$ & $\mathbf{0 , 0 3 4}$ & 0,014 & 0,030 & - \\
\hline
\end{tabular}

Tabelle 27: Hypothese 6, p-Values 
Tabelle 27 stellt das Gesamtbild der Überprüfung der 6. Hypothese auf der Basis der $\mathrm{p}$-Values für beide Planspiele dar.

Drei der sechs Datenreihen weisen in der 3. Periode $p$-Values auf, die mit 0,166, sowie 0,107 und 0,139 oberhalb des gesetzten Signifikanzniveaus von $10 \%$ liegen. Bei strenger Vorgehensweise kann die Nullhypothese in diesen drei Fällen für die 3. Periode auf dem gewählten Signifikanzniveau nicht verworfen werden. Andererseits ist der Sprung der $p$-Values von Periode 2 auf Periode 3 so groß, dass man durchaus von einer Bekräftigung der Annahme 6.a) auch in den drei genannten Fällen ausgehen kann, zumal alle Folgewerte auf eine Fehlerwahrscheinlichkeit von unter $5 \%$ abfallen. Unterstützt wird die Aufrechterhaltung der Annahme 6.a) durch die Betrachtung der Rangsummen bzw. der mittleren Ränge, die hier aus Platzgründen nicht dargestellt werden. Daraus geht überdies die Unterstützung der Annahme 6.b) für alle sechs Datensätze hervor, nach der die Teams der Untersuchungsmenge nach der 2. Periode eine höhere Platzierung erreichen als die Teams der Kontrollmenge.

Auf Basis des Datenmaterials und unter Berücksichtigung der Vorüberlegungen wird daher die 6. Hypothese aufrechterhalten.

Damit ist die empirische Untersuchung abgeschlossen. Sie hat eine Bestätigung von fünf der sechs Hypothesen auf einem Signifikanzniveau von $10 \%$ ergeben, wobei teilweise auch niedrigere Signifikanzniveaus möglich gewesen wären. Die Hypothese 1, in der das Investitionsverhalten untersucht worden ist, konnte nur tendenziell, nicht jedoch auf der Basis des geforderten Signifikanzniveaus bestätigt werden. Da indes die 2. Hypothese, in der die Produktionsmengen-Entscheidungen analysiert werden, enwartungsgemäße Ergebnisse liefert, ist eindeutig festzustellen, dass die Teams der Untersuchungsmenge entsprechend der Strategie der Kostenführung die Produktionsmenge expansiv ausweiten. 407

Obwohl die empirische Untersuchung bei den meisten Datensätzen nur auf einem Signifikanzniveau von $10 \%$ basiert, sprechen zusätzlich zwei weitere qualitative Aspekte für die Unterstützung der Grundhypothese:

407 Zur Strategie der Kostenführerschaft und zur Operationalisierung derselben vgl. 1. Kapitel, Abschnitt 1.1.3.3, 5. Kapitel, Abschnitt 1.5.2 und 7. Kapitel, Abschnitt II.7.3. 
- die ohne Ausnahme zu beobachtende sprunghafte und nachhaltige Veränderung der $p$-Values von Periode 2 auf die Folgeperioden und

- das koordinierte Vorgehen der Teams der Untersuchungsmenge in Bezug auf die expansive Mengenstrategie, gekoppelt mit einem signifikant aggressiveren Marketing.

Insofern kann auf der Basis der empirischen Untersuchungsergebnisse weiterhin von der Grundannahme ausgegangen werden, nach der die Kenntnis von Wettbewerbsstärken und -schwächen auf der Kostenebene, die durch eine Kostenbetrachtung in der Konkurrenzanalyse abgeleitet werden können, strategische Entscheidungen und damit den wirtschaftlichen Unternehmenserfolg beeinflusst.

Die Interpretation und die Bewertung der Untersuchungsergebnisse erfolgt im anschließenden Teil III. Daraus wird die Forderung erhoben, die Kostenbetrachtung als wesentlichen Bestandteil der Konkurrenzanalyse anzusehen. 


\section{Teil III Die Bedeutung der Untersuchungsergebnisse für die Konkurrenzanalyse}

Bevor die Bedeutung der Untersuchungsergebnisse für die Konkurrenzanalyse beurteilt werden kann, ist zunächst im 11. Kapitel die grundsätzliche Allgemeingültigkeit der Ergebnisse zu analysieren. Dabei wird deren Übertragbarkeit auf die Realität, d.h. auf die unternehmerische Praxis erörtert. Die unterschiedlichen empirischen Versuchsergebnisse werden anschließend im 12. Kapitel zusammengeführt und in ihrer Gesamtheit betrachtet und interpretiert. Im 13. Kapitel schließlich werden die Bedeutung der Untersuchungsergebnisse und die daraus resultierende Forderung nach einer festen Berücksichtigung der Kosten von Wettbewerbern im Rahmen der Konkurrenzanalyse abgeleitet. 


\section{Kapitel Allgemeingültigkeit der Untersuchungsergebnisse}

Es ist das Ziel der vorliegenden Studie, möglichst allgemein gültige Aussagen über die Berücksichtigung von Kosten in der Konkurrenzanalyse zu entwickeln. Dementgegen ist festzustellen, dass die empirische Untersuchung mit Hilfe eines Unternehmensplanspiels durchgeführt wird und damit unter den künstlichen Rahmenbedingungen eines Laborexperimentes erfolgt. Um den gewünschten Bezug zur Realität herstellen zu können, muss die Frage nach der allgemein gültigen Aussagefähigkeit der Untersuchungsergebnisse beantwortet werden. $\mathrm{Zu}$ diesem Zweck sind folgende Aspekte und Besonderheiten der Untersuchung zu überprüfen:

1.Umfang und Struktur des Teilnehmerfeldes

2.Werthaltigkeit der Entscheidungsdaten

3.Fokussierung auf Kostenführer

4.Informationsgleichheit

5.Detaillierungsgrad der Information

Im Folgenden werden die genannten Aspekte kritisch erörtert.

\section{III.11.1 Umfang und Struktur des Teilnehmerfeldes}

Bei einer empirischen Untersuchung, die den Anspruch erhebt, allgemein gültige Aussagen über einen bestimmten zu untersuchenden Zusammenhang $\mathrm{zu}$ entwickeln, sind der Umfang und die Beschaffenheit der Stichprobe zu hinterfragen. Damit von einer Stichprobe Rückschlüsse auf eine Grundgesamtheit gezogen werden können, muss die Stichprobe repräsentativ sein, d.h. sie muss ein „verkleinertes Abbild“ der Grundgesamtheit darstellen. ${ }^{408}$

Übertragen auf die vorliegende Studie lässt sich zu beiden Aspekten Folgendes feststellen:

1. Die absolute Teilnehmerzahl: Für empirische Untersuchungen ist eine bestimmte Teilnehmerzahl aus mathematisch-statistischen Gründen notwen-

408 Vgl. HARTUNG, 1999, S. 315. 
dige Voraussetzung. Darüber hinaus lassen sich um so exaktere Rückschlüsse von der Stichprobe auf die Grundgesamtheit ziehen, je größer der Stichprobenumfang ist.

Mit insgesamt ca. 4.000 Personen, die sich - aufgeteilt in 756 Teams - zeitgleich am Planspielwettbewerb beteiligten, kommt mit MARGA bzw. THE BOARD ein Simulationsmodell mit außergewöhnlich hoher Teilnehmerzahl zum Einsatz. Demzufolge stehen ausreichend viele Datensätze für eine mathematisch-statistische Auswertung zur Verfügung. 409

2. Die Struktur des Teilnehmerfeldes: Die Teilnehmer der Studie rekrutieren sich entweder aus Führungskräften, die über Praxiswissen und Erfahrungen verfügen, oder aus Studenten, die sich an den Hochschulen entsprechend aktuelles, theoretisch fundiertes Wissen aneignen. Die Teilnehmerschaft kommt aus einer Vielzahl unterschiedlicher Branchen, Fachrichtungen und Ausbildungsfächem.

Wegen der Bedeutung, die der Beschaffenheit des Teilnehmerfeldes für die Allgemeingültigkeit der Untersuchungsergebnisse beigemessen wird, soll dieser Aspekt im Folgenden näher dargelegt werden. ${ }^{410}$

Im Teilnehmerfeld von MARGA spiegelt sich ein breiter Querschnitt unterschiedlicher Branchen wider, wobei Unternehmen, die dem verarbeitenden Gewerbe zuzuordnen sind, mit $45,0 \%$ die größte Teilnehmergruppe bilden. Der Dienstleistungsbereich umfasst insgesamt $40,2 \%$, davon entfallen $13,4 \%$ auf Finanzdienstleister. Im Vergleich zu den beiden wissenschaftlichen Studien, die bereits mit MARGA durchgeführt wurden, bestätigt sich bei MARGA '99 der von GRÜTTER-SETTELE festgestellte Trend hin zu einem größeren Dienstleistungsanteil411, wie aus Abbildung 30 hervorgeht:

\footnotetext{
409 Die Anzahl der für die einzelnen Hypothesen zugelassenen Datensätze können den Abschnitten II.8.2.1 bis II.8.2.6 im 8. Kapitel entnommen werden.

410 Sowohl FRANZEN als auch GRÜTTER-SETTELE gehen ausführlich auf die demographischen Merkmale der Teilnehmerschaft ein. Vgl. FrAnzen, 1985, S. 119 ff.; GRÜTtER-SETTELE, 1999, S. $93 \mathrm{ff}$.

$411 \mathrm{Vgl.} \mathrm{GRütTER-SetTELE,} \mathrm{1999,} \mathrm{S.} 94$.
} 


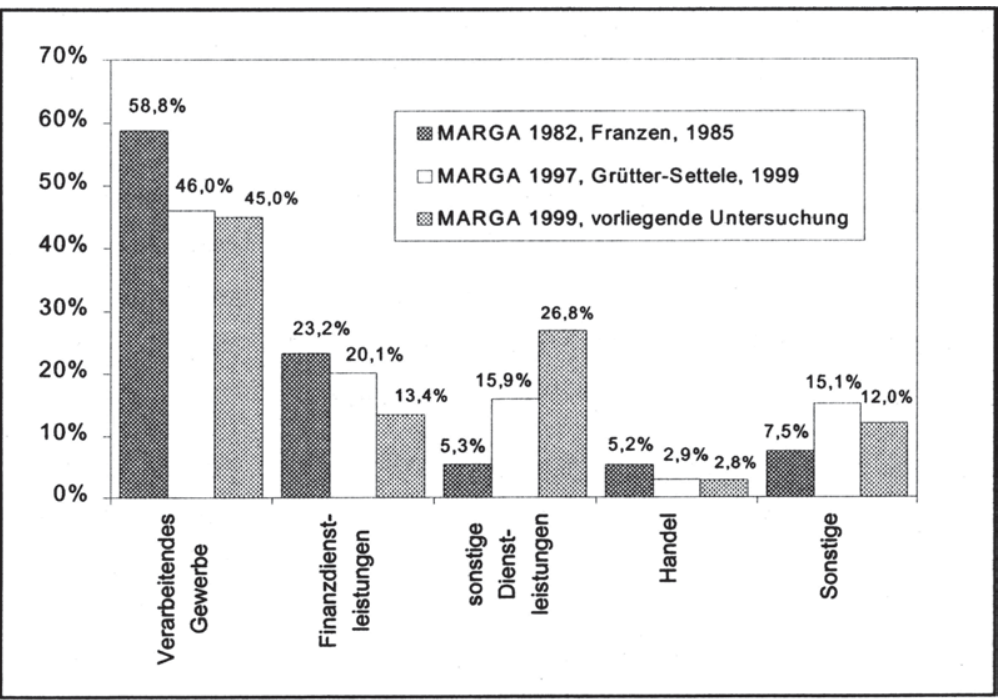

Abbildung 30: Die Branchenzugehörigkeit der MARGA-Teilnehmer

Die Ausbildung der MARGA-Teilnehmer hat sich im Vergleich zu MARGA '97 nur wenig geändert.412 Nach wie vor sind über die Hälfte der MARGA-Teilnehmer nicht wirtschaftswissenschaftlich vorgebildet. Es ergibt sich folgendes Bild:

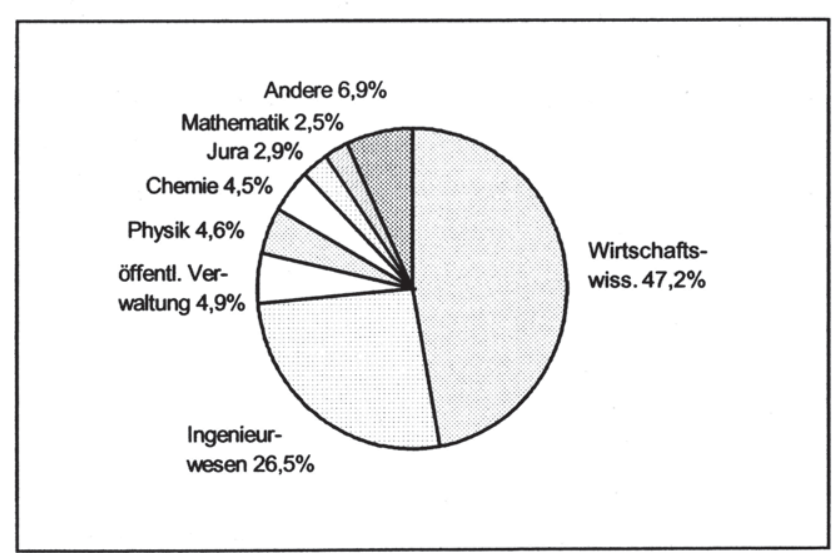

Abbildung 31: Die Ausbildung der MARGA-Teilnehmer

412 Vgl. GRÜtTER-SetTele, 1999, S. 99. 
Bei THE BOARD stellt sich die Teilnehmerzusammensetzung nach Studienrichtung folgendermaßen dar: ${ }^{413}$

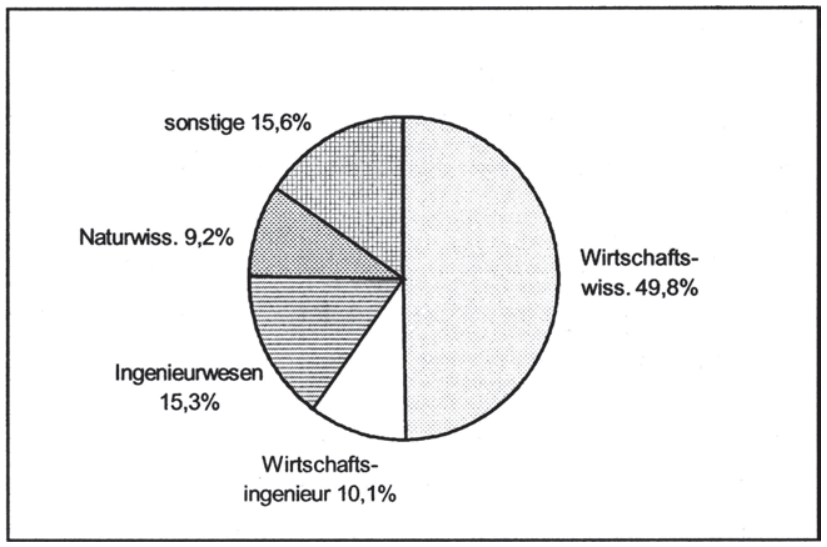

Abbildung 32: Die Studienrichtung der BOARD-Teilnehmer

Für THE BOARD '99 waren Studenten aller Fakultäten und Hochschulen zugelassen, wobei insgesamt 98 verschiedene Hochschulen gemeldet waren. $40 \%$ aller Teams waren interdisziplinär zusammengesetzt, d.h. im Team waren mindestens zwei Fachrichtungen vertreten.

Die Vielfalt der beteiligten Fachrichtungen bei THE BOARD, aber auch die unterschiedliche Vorbildung und der breite Querschnitt durch die Branchen der MARGA-Teams, werden für die vorliegende Studie wegen der Realitätsnähe als Vorteil interpretiert. ${ }^{414}$ Damit kann die Stichprobe bezüglich des Umfanges und der Struktur des Teilnehmerfeldes durchaus als repräsentativ bezeichnet werden, so dass von ihr Rückschlüsse auf die Grundgesamtheit, die Unternehmenspraxis, gezogen werden können.

413 In den naturwissenschaftlichen und in den sonstigen Fächern herrscht bei THE BOARD eine deutlich größere Vielfalt vor, als bei MARGA. Der Anteil einzelner Fächer ist daher gering. Zur besseren Übersicht werden diese Fächer zusammengefasst dargestellt.

414 Wenngleich keine Untersuchung über die Vorbildung von Entscheidungsträgern in realen Unternehmen vorliegt, so geht der Autor davon aus, dass in der Realität in Unternehmen eine ähnliche Vielfalt vorherrscht. 


\section{III.11.2 Werthaltigkeit der Entscheidungsdaten}

Um die Übertragbarkeit der Untersuchungsergebnisse auf die allgemeine Unternehmenspraxis beurteilen zu können, ist zu überprüfen, ob die erhobenen und ausgewerteten Entscheidungsdaten der Teams werthaltig sind, d.h. ob sie entsprechende Aussagekraft besitzen und interpretationsfähig sind. Übertragen auf die vorliegende Untersuchung bedeutet dies, dass die Kosteninformationen der Wettbewerber von einem Großteil der Entscheidungsträger wahrgenommen und bewusst in der Entscheidungsfindung berücksichtigt worden sein sollten. Nur dann basiert die Studie auf werthaltigem Datenmaterial, das als Grundvoraussetzung für die weitere Interpretation anzusehen ist.

Da diese Fragen am besten direkt von den Teams selber beantwortet werden können, wurde im Anschluss an die Versuchsdurchführung, nach dem Ende der Hauptrunde, eine schriftliche Teilnehmerbefragung durchgeführt. Diese methodische Vorgehensweise wurde wegen der Vielzahl der teilnehmenden Teams und aufgrund deren räumlicher Streuung im Fernplanspiel gewählt.

Bei der Teilnehmerbefragung waren ausschließlich die Teams der Untersuchungsmenge zu berücksichtigen, weil nur diese die Kosteninformationen der Wettbewerber erhalten haben. Von insgesamt 384 befragten Teams haben $37 \%$ auswertbare Antworten zurückgesendet, was aus Tabelle 28 hervorgeht:

\begin{tabular}{|c|c|c|c|}
\hline & MARGA & THE BOARD & Summe \\
\hline $\begin{array}{l}\text { Untersuchungs- } \\
\text { menge }\end{array}$ & $\begin{array}{c}36 \text { Gruppe } \\
\hat{=} 144 \text { Teams }\end{array}$ & $\begin{array}{l}60 \text { Gruppen } \\
=240 \text { Teams }\end{array}$ & $\begin{array}{l}96 \text { Gruppen } \\
\triangleq 384 \text { Teams }\end{array}$ \\
\hline $\begin{array}{l}\text { Auswertbare } \\
\text { Antworten }\end{array}$ & $\begin{array}{c}61 \text { Fragebögen } \\
\quad \cong 42 \%\end{array}$ & $\begin{array}{l}81 \text { Fragebögen } \\
\quad \hat{=} 34 \%\end{array}$ & $\begin{array}{c}142 \text { Fragebögen } \\
\quad \approx 37 \%\end{array}$ \\
\hline
\end{tabular}

Tabelie 28: Die auswertbaren Antworten der Teilnehmerbefragung

Insgesamt werden drei Fragestellungen betrachtet, deren Ergebnisse im Folgenden dargestellt werden.

Die erste Frage ermittelt, ob die Teams am Ende der 2. Periode grundsätzlich Kenntnis von den konkurrenzbezogenen Kosteninformationen genommen haben und welche zeitliche Wertigkeit sie diesen eingeräumt haben. Die Frage ist von grundlegender Bedeutung, weil die Wahrnehmung der zur Verfügung ge- 
stellten Kosteninformationen durch die Teilnehmer die Basis des gesamten Experiments darstellt:

1. Wie lange haben Sie sich mit dieser Information beschäftigt?

\begin{tabular}{|l|r|r|r|r|r|r|}
\hline Zeit & \multicolumn{2}{|c|}{ MARGA } & \multicolumn{2}{c|}{ THE BOARD } & \multicolumn{2}{c|}{ Summe } \\
\hline $\mathbf{0}$ min & 1 & $1,6 \%$ & 1 & $1,3 \%$ & 2 & $1,4 \%$ \\
\hline $\mathbf{1 - 1 0} \mathbf{m i n}$ & 35 & $57,4 \%$ & 41 & $50,6 \%$ & 76 & $53,5 \%$ \\
\hline $\mathbf{1 0 - 2 0} \mathbf{m i n}$ & 15 & $24,6 \%$ & 21 & $25,9 \%$ & 36 & $25,4 \%$ \\
\hline$>\mathbf{2 0} \mathbf{m i n}$ & 10 & $16,4 \%$ & 18 & $22,2 \%$ & 28 & $19,7 \%$ \\
\hline Summe & 61 & $100,0 \%$ & 81 & $100,0 \%$ & 142 & $100,0 \%$ \\
\hline
\end{tabular}

Tabelle 29: Die Wahrnehmung der Kosteninformationen

Fast die Hälfte aller betrachteten Teams, 45,1\%, haben sich länger als zehn Minuten mit der Information über die Kostenführer ihrer Branche auseinander gesetzt. Nur insgesamt zwei Teams haben die Informationen nicht bemerkt oder sie als unwichtig eingestuft und sich folglich nicht damit beschäftigt.

Folgende zweite Frage wurde gestellt:

2. Wie schätzen Sie die Bedeutung dieser Information ein?

- Die Information hat uns geholfen, die strategische Situation, in der wir uns befanden, besser einzuschätzen.

\begin{tabular}{|l|r|r|r|r|r|r|}
\cline { 2 - 7 } \multicolumn{1}{c|}{} & \multicolumn{2}{c|}{ MARGA } & \multicolumn{2}{c|}{ THE BOARD } & \multicolumn{2}{c|}{ Summe } \\
\hline Ja & 52 & $85 \%$ & 64 & $79 \%$ & 116 & $82 \%$ \\
\hline Nein & 9 & $15 \%$ & 17 & $21 \%$ & 26 & $18 \%$ \\
\hline Summe & 61 & $100 \%$ & 81 & $100 \%$ & 142 & $100 \%$ \\
\hline
\end{tabular}

Tabelle 30: Die Bedeutung der Kosteninformationen für die strategische Analyse

- Diese Information war Basis für bestimmte Entscheidungen.

\begin{tabular}{|l|r|r|r|r|r|r|}
\cline { 2 - 7 } \multicolumn{1}{c|}{} & \multicolumn{2}{c|}{ MARGA } & \multicolumn{2}{c|}{ THE BOARD } & \multicolumn{2}{c|}{ Summe } \\
\hline Ja & 49 & $80 \%$ & 64 & $79 \%$ & 113 & $80 \%$ \\
\hline Nein & 12 & $20 \%$ & 17 & $21 \%$ & 29 & $20 \%$ \\
\hline Summe & 61 & $100 \%$ & 81 & $100 \%$ & 142 & $100 \%$ \\
\hline
\end{tabular}

Tabelle 31: Die Bedeutung der Kosteninformationen für Entscheidungen 
Für fast alle Teams, denen die Informationen geholfen haben, die eigene Wettbewerbssituation besser einzuschätzen, was für $82 \%$ der betrachteten Teams gilt, war dieses Wissen um Kostenvor- oder -nachteile auch die Basis für bestimmte Entscheidungen.

Die empirische Untersuchung im Teil II hat gezeigt, dass die Teams der Untersuchungsmenge signifikant andere Entscheidungen nach der 2. Periode gefällt haben, als die Teams der Kontrollmenge. Aus den Ergebnissen der ersten beiden Fragen lässt sich ableiten, dass zumindest ein Großteil der Teams der Untersuchungsmenge - es haben sich nur 37\% dieser Teams an der Befragung beteiligt - ihre Entscheidungen unter Einbezug der Kosteninformationen bewusst getroffen haben. Dies wird positiv für die Werthaltigkeit und Interpretationsfähigkeit des erfassten Datenmaterials gewertet.

Die dritte Frage:

3. Halten Sie es aus Ihrer Erfahrung heraus für realistisch, dass man innerhalb einer Branche, die man gut kennt, herausfinden kann, welcher Wettbewerber am günstigsten produzieren kann?

\begin{tabular}{|l|r|r|r|r|r|r|}
\cline { 2 - 7 } \multicolumn{1}{c|}{} & \multicolumn{2}{c|}{ MARGA } & \multicolumn{2}{c|}{ THE BOARD } & \multicolumn{2}{c|}{ Summe } \\
\hline Ja & 48 & $79 \%$ & 65 & $80 \%$ & 113 & $80 \%$ \\
\hline Nein & 13 & $21 \%$ & 16 & $20 \%$ & 29 & $20 \%$ \\
\hline Summe & 61 & $100 \%$ & 81 & $100 \%$ & 142 & $100 \%$ \\
\hline
\end{tabular}

Tabelle 32: Die Beschaffbarkeit von Kosteninformationen

- Wenn ja, können - Ihrer Meinung nach - auch die Ursachen der Kostenvorteile bei Wettbewerbern erschlossen werden?

\begin{tabular}{|l|r|r|r|r|r|r|}
\cline { 2 - 7 } \multicolumn{1}{c|}{} & \multicolumn{2}{c|}{ MARGA } & \multicolumn{2}{c|}{ THE BOARD } & \multicolumn{2}{c|}{ Summe } \\
\hline Ja & 40 & $83 \%$ & 57 & $88 \%$ & 97 & $86 \%$ \\
\hline Nein & 8 & $17 \%$ & 8 & $12 \%$ & 16 & $14 \%$ \\
\hline Summe & 48 & $100 \%$ & 65 & $100 \%$ & 113 & $100 \%$ \\
\hline
\end{tabular}

Tabelle 33: Die Informationen über die Ursachen von Kostenvorteilen

$80 \%$ der Teams halten es für realistisch, Kosteninformationen von Wettbewerbern zu erhalten. Von diesen 113 Teams glauben 97 Teams, dass man auch die Ursachen von Kostenvorteilen herausfinden kann. Dieses Ergebnis ent- 
spricht den eher theoretischen Ausführungen im 4. Kapitel, Abschnitt I.4.2, in denen diese Möglichkeit ebenfalls angenommen wird.

Obwohl die in der dritten Frage betrachtete persönliche Einschätzung der Teams sicherlich nicht repräsentativ ist und somit nicht auf die allgemeine Unternehmenspraxis übertragen werden kann, spiegelt sie doch die Erfahrung von 142 Teams wider, die sich im Planspielwettbewerb über mehrere Monate mit dieser Problematik auseinander gesetzt haben.

Auch wenn sich nur $37 \%$ aller Teams an der Teilnehmerbefragung beteiligt haben und daher die Ergebnisse nicht uneingeschränkt auf alle Probanden übertragen werden können, so ist für die vorliegende Studie doch davon auszugehen, dass ein Großteil der Teams die Kosteninformationen der Wettbewerber wahrgenommen und bewusst in der Entscheidungsfindung berücksichtigt hat. Daraus wird abgeleitet, dass die zugrundeliegenden Entscheidungsdaten werthaltig und interpretationsfähig sind. Dafür sprechen zusätzlich die signifikanten Untersuchungsergebnisse ${ }^{415}$, sowie die Motivation der Teilnehmer ${ }^{416}$.

\section{III.11.3 Fokussierung auf Kostenführer}

Im empirischen Teil der Studie stehen ausschließlich strategische Entscheidungen und daraus resultierender wirtschaftlicher Erfolg von Unternehmen im Vordergrund, die sich innerhalb ihres Wettbewerbsumfeldes in kostenführender Position befinden.

Die allgemeine Übertragbarkeit der Untersuchungsergebnisse auf die Unternehmenspraxis wird dadurch jedoch nur gering eingeschränkt, da sich aus der vorliegenden Untersuchung auch Rückschlüsse auf Nicht-Kostenführer ziehen lassen. Allerdings werden diese nicht empirisch untersucht und entsprechend gestützt, sondern „nur” aus den übrigen Erkenntnissen abgeleitet.

So ist die Kenntnis eigener Wettbewerbsstärken auf der Kostenebene besonders für Unternehmen relevant, die den Kostenvorsprung in den Mittelpunkt

415 Vgl. 10. Kapitel, Abschnitt II.10.1 bis II.10.6.

416 Vgl. 7. Kapitel, Abschnitt II.7.2. 
ihrer strategischen Ausrichtung stellen und dabei eine Kostenführung anstreben. ${ }^{417}$ Diese Unternehmen können im verwendeten Planspielmodell ihr Wissen um eigene Kostenvorteile in strategische Überlegungen einbeziehen und gezielt nutzen und werden daher in der Studie empirisch untersucht.

Ähnliches gilt für Unternehmen, deren strategische Ausrichtung und deren wirtschaftlicher Erfolg eher auf einer Differenzierung oder auf der Besetzung einer Marktnische aufbauen und die den strategischen Fokus nicht auf Kostenvorteile, sondern bspw. eher auf qualitative Produkt- oder Leistungsmerkmale oder auf regionale oder technische Nischen setzen. Auch in diesen Fällen nimmt deren Planungs- und Entscheidungssicherheit zu, wenn sie eigene Kostennachteile kennen und diese strategisch entsprechend berücksichtigen können.

Nun ist es nicht Ziel der Studie herauszufinden, ob die Kenntnis von Wettbewerbsvorteilen oder -nachteilen auf der Kostenebene für Kostenführer von gröBerer Wichtigkeit ist, als für Unternehmen, die sich anders strategisch positionieren. Vielmehr wird folgender Frage nachgegangen: Sind Kosteninformationen über Konkurrenzunternehmen strategisch bedeutend und sollten sie daher ein fester Bestandteil einer Konkurrenzanalyse sein? Dabei wird untersucht, ob und inwiefern die Kenntnis eigener Wettbewerbsstärken oder -schwächen auf der Kostenebene grundsätzlich vorteilhaft ist, ob dieses Wissen in den strategischen Entscheidungsprozess einfließt und ob dadurch die Entscheidungsqualität beeinflusst wird.

\section{III.11.4 Informationsgleichheit}

Allen Teams einer Vierergruppe werden zum selben Zeitpunkt identische Informationen zur Verfügung gestellt. Dieses Vorgehen ist nicht realistisch, da im Regelfall von einem mehr oder weniger großen Informationsvorsprung einzelner Wettbewerber ausgegangen werden kann.

Ein Informationsvorsprung in einem für ein Unternehmen strategisch relevanten Bereich, der in der Realität durch eine entsprechend gestaltete Konkurrenzanalyse erreichbar sein könnte, stellt einen Vorteil für das betreffende Unter-

417 Vgl. 1. Kapitel, Abschnitt I.1.3.3 und 5. Kapitel, Abschnitt I.5.2. 
nehmen dar. Übertragen auf die vorliegende Studie bedeutet diese Aussage Folgendes: Wenn Informationen über die Wettbewerbsstärke auf der Kostenebene für kostenführende Unternehmen strategierelevant sind, wovon hier aufgrund der Untersuchungsergebnisse ausgegangen werden soll, dann haben Kostenführer, die als einziges Unternehmen innerhalb ihrer Vierergruppe ihre günstige Kostenposition kennen, einen Vorteil gegenüber den Kostenführern, denen diese Informationen nicht exklusiv zur Verfügung stehen, sondern von denen alle Konkurrenten wissen, dass sie Kostenführer sind.

Demzufolge trägt die Informationsgleichheit in dieser Studie dazu bei, eindeutige Ursache-Wirkungs-Zusammenhänge frei von Störgrößen, wie bspw. einem Informationsvorsprung, nachzuweisen. Wenn die Kostenführer der Untersuchungsmenge einen Informationsvorsprung bezüglich ihrer Kostenposition erhalten würden, ist davon auszugehen, dass sie diesen zusätzlichen Vorteil nutzen und die Untersuchungsergebnisse eine noch deutlichere Unterstützung der Grundhypothese zeigen würden. Insofern stellt das gewählte Vorgehen keine Einschränkung der Allgemeingültigkeit dar, da selbst bei Informationsgleichheit die Grundhypothese unterstützt wird.

\section{III.11.5 Detaillierungsgrad der Information}

In der vorliegenden Untersuchung wird nur die grundsätzliche Information über das kostenführende Unternehmen je Produkt bekannt gegeben. Detaillierte Informationen über die Kostensituation der Wettbewerber, wie bspw. die Deutlichkeit des Kostenvorsprungs bleiben ebenso unberücksichtigt, wie z.B. die Overhead-Kosten der Unternehmen.

Durch die Reduktion der Kosteninformation ausschließlich auf die Nennung des kostenführenden Unternehmens wird ebenfalls keine Einschränkung der Allgemeingültigkeit der Untersuchungsergebnisse, sondern eher das Gegenteil verursacht. Denn es ist davon auszugehen, dass detailliertere Kosteninformationen von Wettbewerbern, wie bspw. ein exakt quantifizierter Kostenvorsprung, schwieriger zu erhalten sind, als die vergleichsweise einfach zu beschaffende 
Grundaussage darüber, welches Unternehmen einer Branche sich in einer kostenführenden Position befindet. ${ }^{418}$

Im MARGA-Modell setzen sich die variablen Kosten, über die die Kostenführer in dieser Untersuchung bestimmt werden, aus inhaltlichen und didaktischen Gründen ausschließlich aus den Material- und Personalkosten zusammen. Die Overhead-Kosten, die in der Realität von erheblicher Bedeutung sind, bleiben unberücksichtigt. In der vorliegenden Studie bestätigt sich jedoch die Grundhypothese selbst auf der Basis des einfacheren Informationsniveaus.

Die in diesem Kapitel erörterte Frage nach der allgemein gültigen Aussagefähigkeit der Untersuchungsergebnisse lässt sich nicht zweifelsfrei beantworten. Schließlich kann in der vorliegenden Studie nicht die gesamte Wirklichkeit erfasst, sondern "nur" ein Experiment auf der Basis einer repräsentativen Stichprobe durchgeführt werden, mit deren Hilfe Rückschlüsse auf die Grundgesamtheit gezogen werden sollen. Die modellhafte Abbildung eines Unternehmens reduziert die Komplexität, was zu Vor- und Nachteilen führt: Einerseits fehlt einem Modell die Komplexität der Realität, andererseits lassen sich jedoch ceteris paribus eindeutige Ursache-Wirkungs-Zusammenhänge ableiten, auf deren Basis allgemein gültige Regeln oder Gesetzmäßigkeiten erarbeitet werden können, wie es in der vorliegenden Studie der Fall ist.

418 Die Informationsgewinnung in der Konkurrenzanalyse wird im 4. Kapitel behandelt. 


\section{Kapitel Interpretation der Untersuchungsergebnisse}

Basierend auf der Grundhypothese sind insgesamt sechs Einzelhypothesen formuliert und empirisch untersucht worden. Die im 10. Kapitel dargestellten Ergebnisse werden im Folgenden aufgegriffen und interpretiert.

In den Hypothesen 1 bis 5 stehen fünf Entscheidungsprobleme im Mittelpunkt, die bei der Umsetzung einer Strategie der Kostenführung von den Teams zu analysieren und entsprechend zu bearbeiten sind:419 Die Hypothesen 1 und 2 fokussieren den Investitions- bzw. Produktionsbereich. Die Hypothesen 3 bis 5 , welche die Preispolitik, die Forschungs- und Entwicklungsaktivitäten - und damit bei MARGA die Produktqualität - sowie die Werbeintensität der Planspielunternehmen erfassen, decken den Marketingbereich ab. Da die Realisierung der Kostenführerstrategie sowohl eine Ausweitung der Produktionsmenge, als auch eine entsprechend aggressive Marketingstrategie erfordern, sind von den Planspiel-Teams koordinierte Entscheidungen in beiden Unternehmensbereichen zu fällen.

Mit Hilfe mathematisch-statistischer Verfahren können vier der fünf Hypothesen bestätigt werden. ${ }^{420}$ Die 1. Hypothese, bei der die Investitionsaktivitäten der Unternehmen untersucht werden, kann zwar tendenziell, nicht jedoch auf dem geforderten Signifikanzniveau aufrecht erhalten werden. Parallel dazu erhöht sich, gemäß der 2 . Hypothese, die Produktionsmenge signifikant bei den Unternehmen der Untersuchungsmenge im Vergleich zu den Teams der Kontrollmenge. Offensichtlich konnten die Unternehmen der Untersuchungsmenge teilweise auf freie Kapazitätsreserven zurückgreifen, wodurch die Investitionsaktivitäten geringer als erwartet ausgefallen sind. Trotz dieser unerwarteten Entwicklung spricht die bei den Teams der Untersuchungsmenge festzustellende signifikant erhöhte Produktionsmenge für eine Umsetzung der Kostenführerstrategie.

Das Ergebnis aus dem Produktionsbereich bestätigt sich im Marketingbereich, der durch die Hypothesen 3 bis 5 abgebildet wird. Hat sich ein Team, wie in den Hypothesen 1 und 2 untersucht, für eine Erhöhung der Kapazitäten bzw. der

419 Im 8. Kapitel, Abschnitt II.8.2, werden die Hypothesen ausführlich dargestellt.

$420 \mathrm{Im}$ 9. Kapitel werden diese Verfahren dargestellt.

Christoph Heinen - 978-3-631-75315-6 
Produktionsmenge entschieden, steht es unter dem Zwang, die erhöhte Menge auf dem Markt abzusetzen. Dementsprechend wählen die Teams der Untersuchungsmenge ein signifikant aggressiveres Marketing, das mit der Erhöhung der Produktionsmenge korrespondiert und zu einem Absatzwachstum führen soll.

Aus diesem koordinierten Vorgehen im Produktions- und Marketingbereich lässt sich ableiten, dass die Teams der Untersuchungsmenge, die über ihre Wettbewerbsstärken auf der Kostenebene informiert waren, dieses Wissen genutzt haben, indem sie die Kostenführung als strategische Grundausrichtung eingeschlagen haben. Insofern hat die Kenntnis der Wettbewerbsstärken auf Kostenebene das strategische Handeln der Entscheidungsträger beeinflusst.

Das Ergebnis des Handelns kommt im wirtschaftlichen Erfolg zum Ausdruck. Dabei stellt sich die Frage, ob das Wissen um Wettbewerbsstärken auf der Kostenebene die Unternehmen nicht nur dazu veranlasst, bestimmte strategische Entscheidungen zu treffen, sondern sie zusätzlich dazu befähigt, Chancen zu erkennen, zu ergreifen und in erfolgreiche Strategien umzusetzen. Zur Beantwortung dieser Fragestellung wird in der 6. Hypothese der wirtschaftliche Erfolg der Planspiel-Unternehmen betrachtet, der sich in einer entsprechenden Platzierung des MARGA-Unternehmens innerhalb seiner Vierergruppe zeigt.

Größtenteils auf einem Signifikanzniveau von weniger als $5 \%$ lässt sich in Hypothese 6 feststellen, dass die über ihren Kostenvorteil informierten Kostenführer wirtschaftlich erfolgreicher abschneiden, als die nicht informierten Teams. Offensichtlich hat die Kenntnis der eigenen Wettbewerbsstärke auf der Kostenebene die Unternehmen der Untersuchungsmenge in einem signifikant nachweisbaren Umfang dazu in die Lage versetzt, eine wirtschaftlich erfolgreiche Wettbewerbsstrategie, in diesem Fall die Kostenführung, zu finden und zu verfolgen.

Da die einzelnen Hypothesen aus der Grundhypothese abgeleitet sind ${ }^{421}$, wird diese durch die Untersuchungsergebnisse bestätigt. Danach führt die Kenntnis der Stärken und Schwächen auf der Kostenebene, welche durch eine Kostenbetrachtung in der Konkurrenzanalyse abgeleitet werden können, zu einer Zu-

421 Die Herleitung der Grundhypothese erfolgt im 5. Kapitel, Abschnitt I.5.2, während die sechs Einzelhypothesen daraus im 8. Kapitel, Abschnitt II.8.2, abgeleitet werden. 
nahme an Planungs- und Entscheidungssicherheit. Die Wettbewerbsstärke wird als solche erkannt und offensichtlich von den Entscheidungsträgern strategisch genutzt. Dies spiegelt sich in den Entscheidungen der betroffenen Unternehmen und damit auch im wirtschaftlichen Erfolg wider.

Im Gegensatz dazu wird die strategische Chance von Entscheidungsträgern, die sich in der gleichen günstigen Kostenposition befinden, die jedoch von ihrer Stärke auf der Kostenebene nichts wissen, auch nicht erkannt. Sie entscheiden sich entweder für andere Strategien, die ihren Wettbewerbsvorteil auf der Kostenebene nicht in vollem Umfang ausnutzen, oder für eine Kostenführerstrategie, die sie jedoch nicht mit dem gleichen Nachdruck umsetzen, wie die Kostenführer, die sich ihres Vorteils bewusst sind. Entsprechend weniger positiv wirkt sich die Unkenntnis bzw. die Unsicherheit bezüglich eigener Wettbewerbsstärken und -schwächen auf den wirtschaftlichen Erfolg aus.

Der Kenntnis von Wettbewerbsstärken auf der Kostenebene kommt nach den vorliegenden Untersuchungsergebnissen eine wichtige Bedeutung zu. Dabei sind numerisch exakte Kosteninformationen, wie die vorliegende empirische Studie gezeigt hat, offensichtlich weniger entscheidend, als vielmehr die meist leichter zu erlangende Erkenntnis vergleichender Vor- oder Nachteile auf der Kostenebene. Erst dieses Wissen eröffnet strategische Chancen, die nur nutzbar sind, wenn sie erkannt werden. 


\section{Kapitel Kostenbetrachtung von Wettbewerbern als ein fester Bestandteil der Konkurrenzanalyse}

Die durchgeführte Studie zeigt, dass Kosteninformationen von Wettbewerbern strategische Entscheidungen und damit den wirtschaftlichen Erfolg eines Unternehmens beeinflussen. Daraus abgeleitet stellt sich die grundsätzliche Frage nach der Berücksichtigung von Kosteninformationen in der Konkurrenzanalyse, die im 13. Kapitel abschließend positiv beantwortet wird.

Der Analysephase als Teil des strategischen Planungsprozesses wird in der Literatur ein hoher Stellenwert beigemessen, da sie die Grundlage der Strategieformulierung und -auswahl bildet. ${ }^{422}$ Die Konkurrenzanalyse ist im marktwirtschaftlichen System ein wesentliches Element dieser Analysephase. Die Inhalte richten sich vorwiegend nach der jeweiligen Wettbewerbssituation, in der sich das Unternehmen befindet und nach den individuellen Informationsbedürfnissen der Entscheidungsträger. Einbezogen werden sollten demnach alle Konkurrenzinformationen, die "für eigene Entscheidungen im Rahmen der strategischen Unternehmensplanung von Bedeutung sind“423.

Im Gegensatz zu den Erkenntnissen dieser Untersuchung, nach denen Kosteninformationen von Wettbewerbern durchaus strategierelevant sind und demnach im Rahmen der Konkurrenzanalyse erfasst und verarbeitet werden sollten, bewertet das Schriftum die Berücksichtigung von Kosten in der Konkurrenzanalyse kontrovers. Während einige Autoren Kosten von Wettbewerbern gar nicht berücksichtigen, erlangen sie bei anderen Autoren eine mehr oder weniger stark ausgeprägte Bedeutung. Dabei lassen sich, wie bereits an anderer Stelle dargestellt, insgesamt drei Abstufungen unterscheiden. ${ }^{424}$

Kosteninformationen in der Konkurrenzanalyse zu vernachlässigen, wie dies bei Autoren der ersten Kategorie der Fall ist, heißt, Stärken oder Schwächen auf der Kostenebene gar nicht erst erkennen zu können. Die Planungs- und Entscheidungssicherheit nimmt ab und die Wahrscheinlichkeit dafür, dass Stärken auf der Kostenebene ungenutzt bleiben, nimmt zu. Da auch Kostennachteile

422 Vgl. 1. Kapitel, Anschnitt I.1.3.2.

423 KREIKEBAUM, 1997, S. 121.

424 Vgl. 5. Kapitel, Abschnitt 1.5.1. 
nicht erkennbar sind, werden strategische Entscheidungen auf der Basis eines unvollständigen Stärken-/Schwächen-Profils getroffen, wodurch das Risiko von Fehlentscheidungen wächst.

Beziehen parallel dazu die Wettbewerber Kosteninformationen ihrer Konkurrenten in ihre strategischen Überlegungen ein, kommt zu der geschilderten Problematik eines unvollständigen Stärken-/Schwächen-Profils und der daraus resultierenden höheren Wahrscheinlichkeit von Fehlentscheidungen auch ein Informationsnachteil hinzu. Obwohl die Auswirkungen eines Informationsunterschieds auf der Kostenebene bei Wettbewerbern im Rahmen dieser Studie nicht untersucht werden, lässt sich doch feststellen, dass ein Informationsnachteil für das betreffende Unternehmen sicherlich keine Vorteile, sondern eher negative Konsequenzen aufweist.

Demgegenüber werden Kosteninformationen von Autoren der zweiten und dritten Kategorie im Rahmen der Konkurrenzanalyse berücksichtigt. Zwar erfolgt dies mit einer unterschiedlichen Gewichtung, doch ist hier entscheidend, dass die Kosteninformationen überhaupt erfasst und beachtet werden. Auf diese Weise ist sichergestellt, dass brancheninterne Kostenvor- oder -nachteile erkannt werden. Unabhängig von der grundsätzlichen Kenntnisnahme kann anschließend die Wichtigkeit dieser Informationen in Abhängigkeit von der spezifischen Wettbewerbssituation beurteilt und entsprechend der individuellen Bedeutung mehr oder weniger stark berücksichtigt werden.

Die Kenntnis eigener Kostenvorteile führt, wie bereits dargestellt, zu einer Zunahme an Planungs- und Entscheidungssicherheit. Eigene Stärken auf der Kostenebene können - wenn sie als solche erkannt werden - mit der entsprechenden Nachdrücklichkeit erfolgreich genutzt werden, wie die empirische Untersuchung gezeigt hat.

Andererseits lassen sich eigene Schwächen bzw. Wettbewerbsnachteile auf der Kostenebene durch eine frühzeitige alternative strategische Ausrichtung in ihrer Gefahr für das Unternehmen abschwächen oder entschärfen. Dabei signalisiert die Erkenntnis eigener Kostennachteile einen Handlungsbedarf und erzeugt einen oft nicht unerwünschten Handlungsdruck, welcher durch die zunehmende Bedeutung wertorientierter Grundsätze in der Unternehmensführung flankiert 
wird. 425 In dieser Situation ist in einem ersten Schritt eine detaillierte Erfassung der Kosten zu empfehlen, bei der mit Hilfe der bereits beschriebenen Wertkettenanalyse eine nach Wertaktivitäten differenzierte Kostenbetrachtung erfolgen sollte.426 Die daraus abgeleiteten und nach einzelnen Aktivitäten differenzierten Kostenvor- und -nachteile können anschließend - neben einem allgemein stärkeren Kostenbewusstsein - zu einer Selektion und Ausgliederung einzelner Aktivitäten, Abteilungen oder ganzer Bereiche oder zu einer völligen strategischen Neuausrichtung des Unternehmens führen, bei der die Kostenposition eine untergeordnete strategische Rolle spielt. ${ }^{427}$ Wichtig ist auch in diesem Fall, sich der Wettbewerbssituation, in der man sich befindet, möglichst frühzeitig bewusst zu sein, um geeignete Gegenmaßnahmen ergreifen zu können. Je früher dies geschehen kann, desto größer werden im Allgemeinen die Erfolgsaussichten eingeleiteter Maßnahmen sein.

Die Kenntnis von Kostenvor- und -nachteilen bestimmter Wettbewerber ermöglicht es, deren zukünftiges Verhalten mit einer größeren Wahrscheinlichkeit zu antizipieren. So lässt sich bspw. abschätzen, welche Konkurrenten im Falle einer eigenen Preissenkung kurzfristig nachziehen könnten und inwieweit damit die Gefahr einer Spirale von Preissenkungen bestünde. Zwar spielen in diesem Beispiel auch viele andere Aspekte, wie evtl. in der Branche vorhandene und auszulastende Kapazitäten oder die damit eng verbundene Problematik der Kostenflexibilität bei Beschäftigungsrückgängen eine Rolle, doch lassen sich auf diese Weise mögliche Reaktionsprofile einzelner Wettbewerber bis hin zu Branchenszenarien im Vorfeld eigener Entscheidungen durchdenken.

Wenn Kosteninformationen von Wettbewerbern strategisch derart bedeutend sind, wie es sich in der vorliegenden Studie darstellt und wie es aus den begleitenden Überlegungen abzuleiten ist, sind sie im Rahmen der Konkurrenzanalyse zu berücksichtigen. Insofern ist den Autoren der zweiten und dritten Kategorie im oben dargestellten Spektrum zuzustimmen, während den Autoren

$425 \mathrm{Zu}$ den Grundsätzen und den Handlungsmaximen der wertorientierten Unternehmensführung, bzw. des Shareholder Value-Managements vgl.: FrANZ/KAJÜTER, 2000 (b), S. $200 \mathrm{ff}$.

426 Vgl. 3. Kapitel, Abschnitt I.3.6.

427 Zur Neugestaltung einer wettbewerbsfähigeren Wertkette vgl. BAUR/KLUGE, 2000, S. $138 \mathrm{ff}$. 
der ersten Kategorie, nach der Kosteninformationen von Wettbewerbern nicht berücksichtigt werden, nicht zugestimmt werden kann. 428

Werden Kosteninformationen von Wettbewerbern im Rahmen der Konkurrenzanalyse erfasst, so stellt sich die weiterführende Frage, ob diese entsprechend der Kategorien zwei und drei nur als einfacher, zu überprüfender, oder als Bestandteil mit wesentlicher strategischer Relevanz zu sehen sind. Dies hängt von der strategischen Ausrichtung und von der individuellen Wettbewerbssituation $a b$, in der sich ein Unternehmen befindet. Allgemein lässt sich feststellen, dass die Kostenbetrachtung in der Konkurrenzanalyse um so bedeutender ist, je zentraler die Kostenposition für das Unternehmen und seine strategische Positionierung ist. Die individuelle Wettbewerbssituation kann mit Hilfe der bereits dargestellten fünf Wettbewerbskräfte analysiert werden ${ }^{429}$, wobei gilt, je dynamischer das Wettbewerbsumfeld, desto ratsamer ist die regelmäßige und ausführliche Betrachtung der Wettbewerbskosten. An dieser Stelle ergibt sich weiterführender Forschungsbedarf, bei dem die geschilderte Abhängigkeit vom Einzelfall zu strukturieren und zu systematisieren ist.

Zusammenfassend leitet sich aus den Untersuchungsergebnissen und aus den daran anknüpfenden, weiterführenden Überlegungen die Forderung ab, eine Kostenbetrachtung als festen Bestandteil einer Konkurrenzanalyse im Rahmen des strategischen Planungsprozesses anzusehen, den Detaillierungsgrad und den Einfluss auf Entscheidungen jedoch vom noch zu erforschenden Einzelfall abhängig zu machen.

428 Vgl. 5. Kapitel, Abschnitt I.5.1.

429 Vgl. 1. Kapitel, Abschnitt I.1.3.2. 
Christoph Heinen - 978-3-631-75315-6

Downloaded from PubFactory at 01/11/2019 06:18:34AM

via free access 


\section{Zusammenfassung}

Die Konkurrenzanalyse steht im Mittelpunkt dieser Arbeit. Sie ist Teil des strategischen Planungsprozesses und gehört darin zur Analysephase. Die analytische Betrachtung des Unternehmens und seiner Umwelt hat einen weichenstellenden Einfluss auf die strategische Ausrichtung des Unternehmens und ist damit von größter Bedeutung für alle Unternehmensbereiche.

In der Konkurrenzanalyse werden unterschiedliche Merkmale der relevanten und potentiellen Wettbewerbsunternehmen verarbeitet. Die zu untersuchenden Inhalte richten sich danach, inwiefern sie im Rahmen der strategischen Unternehmensplanung von Bedeutung sind. Bei der Konkretisierung der zu erfassenden Informationen ist im Schriftum eine unterschiedliche Auffassung über die Berücksichtigung von Kosten in der Konkurrenzanalyse festzustellen: Während eine Gruppe von Autoren Kosten von Wettbewerbern nicht beachtet, werden sie von zwei weiteren Gruppen erfasst, jedoch in unterschiedlicher Intensität in die weiterführenden Überlegungen einbezogen.

Diese Kontroverse aufgreifend, wird in der vorliegenden Arbeit die Bedeutung der Kostenbetrachtung in der Konkurrenzanalyse untersucht. Zu diesem Zweck wird eine Grundhypothese formuliert, nach der die Kenntnis der Stärken und Schwächen auf der Kostenebene, welche durch eine Kostenbetrachtung in der Konkurrenzanalyse abgeleitet werden können, zu einer Zunahme an Planungsund Entscheidungssicherheit führt. Dies sollte sich sowohl in strategischen Entscheidungen, als auch im wirtschaftlichen Erfolg informierter Unternehmen positiv widerspiegeln.

Um die in der Grundhypothese zusammengefassten, komplexen Zusammenhänge möglichst realistisch überprüfen zu können, sollten in einer empirischen Untersuchung tatsächliche Entscheidungen von Wirtschaftssubjekten zu Grunde gelegt werden. Diese können mit Hilfe des General Management Planspiels MARGA in der erforderlichen Qualität und Quantität generiert und erfasst werden. Ausgehend von der Grundhypothese werden sechs Einzelhypothesen abgeleitet, wodurch sich die Problemstellung in ihrer Komplexität reduziert und die einzelnen Aspekte der zu untersuchenden Zusammenhänge differenziert analysiert werden können. 
Die empirische Untersuchung fokussiert Unternehmen, die sich in kostenführender Position befinden. Nur ca. $50 \%$ aller betrachteten Kostenführer werden im Planspielverlauf über ihre im Vergleich zu den Wettbewerbern günstige Kostenposition informiert. Anschließend werden eine Reihe strategischer Entscheidungen und der wirtschaftliche Erfolg aller Kostenführer erfasst und im Rahmen der sechs Hypothesen miteinander verglichen. Dabei zeigen sich signifikante Unterschiede zwischen den informierten und den nicht informierten Kostenführern. Hieraus lässt sich ableiten, dass die Kenntnis von Wettbewerbsstärken auf der Kostenebene einen Einfluss auf strategische Entscheidungen hat. Da auch der wirtschaftliche Erfolg der informierten Unternehmen signifikant größer ist, kann davon ausgegangen werden, dass die Kostenführer, die sich ihrer Stärke bewusst waren, durch diese Kenntnis in die Lage versetzt wurden, Chancen zu erkennen und strategisch zu nutzen. Insofern bestätigt die empirische Untersuchung die aufgestellte Grundhypothese.

Von diesen Erkenntnissen ausgehend, lassen sich - allerdings nicht empirisch untermauert - auch Rückschlüsse auf Unternehmen ziehen, die sich nicht in kostenführender Position befinden. Denn wird die Kostenbetrachtung der Wettbewerber nicht durchgeführt, bleiben sowohl Stärken als auch Schwächen auf der Kostenebene unerkannt, was in jedem Fall die Planungs- und Entscheidungssicherheit reduziert und das Risiko von Fehlentscheidungen, die in diesem Zusammenhang eine strategische Tragweite haben, erhöht. Hinzu kommt ein Informationsnachteil, falls Konkurrenten die relative Kostensituation ermitteln und berücksichtigen.

Das Erkennen von einzelnen Kostenvor- und -nachteilen setzt eine entsprechend detaillierte Kostenanalyse der Wettbewerber voraus. Diese sollte organisatorisch im Bereich der Konkurrenzanalyse angesiedelt sein, da hier die notwendige breite Informationsbasis vorhanden ist und die gewonnenen Erkenntnisse direkt im strategischen Planungsprozess weiterverarbeitet werden. Da Kosteninformationen von Wettbewerbern gewöhnlich als "sensibel“ eingestuft werden und sie damit nicht direkt zu erfassen sind, lassen sich diese nur durch eine systematische und institutionalisierte Konkurrenzanalyse beschaffen. Durch das Zusammenfügen vieler Einzelinformationen und durch den Einsatz diverser Hilfsinstrumente, wie z.B. der Wertkettenanalyse oder der Erfahrungskurve, die jeweils andere Blickwinkel eröffnen, sollten durch den Vergleich mit 
dem eigenen Unternehmen Rückschlüsse auf die Kostenstrukturen von Konkurrenten gezogen werden können. Meist werden dabei nicht nur vergleichende qualitative Aussagen, sondern zumindest näherungsweise auch quantitative Erkenntnisse ableitbar sein.

Die heutige hoch entwickelte, eng vernetzte und in vielen Branchen stark arbeitsteilige Wirtschaftsstruktur ermöglicht es aufgrund kommunikationstechnischer und logistischer Fortschritte, einzelne Kostennachteile im Wertschöpfungsprozess gezielt auszugleichen. Durch Outsourcing, teilweises Ausgliedern bestimmter Arbeitsschritte oder durch den Zukauf von Zwischenprodukten lassen sich auch einzelne Wertschöpfungsaktivitäten abbauen, die von anderen Unternehmen bspw. kostengünstiger durchgeführt werden können. Das Ziel ist eine für den Kunden optimale Gestaltung des Wertschöpfungsprozesses, wobei eigene Aktivitäten mit fremdbezogenen Leistungen oder Gütern sinnvoll, d.h. auch kostenoptimal, kombiniert werden. Die Voraussetzung dafür ist das Erkennen eigener und fremder Wettbewerbsvor- oder -nachteile, bspw. auf der Kostenebene.

Wie in dieser Arbeit zum Ausdruck kommt, eröffnen die Erkenntnisse einer Kostenanalyse von Wettbewerbsunternehmen bedeutende Chancen und zwar unabhängig von der eigenen relativen Kostenposition. Während das Wissen um Wettbewerbsstärken auf der Kostenebene ein selbstbewusstes und nachdrückliches strategisches Handels begünstigt, schafft das Wissen um Kostennachteile strategischen, aber auch operativen Handlungsdruck.

Auf der Basis der vorliegenden Studie lässt sich daher die Berücksichtigung von Kosten in der Konkurrenzanalyse ausdrücklich empfehlen. 
Christoph Heinen - 978-3-631-75315-6

Downloaded from PubFactory at 01/11/2019 06:18:34AM

via free access 


\section{Literaturverzeichnis}

ANSOFF, H.I.: Strategies for Diversification, in: HBR, 1957, Vol. 35, No. 5.

ANSOFF, H.I.: The New Corporate Strategy, New York, 1987.

BAUR, C.; KLUGE, J.: Die Wertkette als Instrument der strategischen Analyse, in:

Welge, M.K.; Al-Laham, A.; KaJüter, P. (Hrsg.): Praxis des Strategischen

Managements, Konzepte - Erfahrungen - Perspektiven, Wiesbaden, 2000.

Berekoven, L.; ECKert, W.; Ellenrieder, P.: Marktforschung. Methodische Grundlagen und praktische Anwendungen, 7. Aufl., Wiesbaden, 1996.

BIEG, H.; KUBMAUL, H.: Externes Rechnungswesen, München, 1996.

BöHLER, H.: Marktforschung, 2. Aufl., Stuttgart, Berlin, Köln, 1992.

BÜHL, A.; ZÖFEL, P.: SPSS Version 8, Bonn, 1999.

BÜNING, H.; TRENKLER, G.: Nichtparametrische statistische Verfahren, 2. Aufl., Berlin,1994.

Busse von Colbe, W.: Rechnungswesen, in: Busse von Colbe, W.; Pellens, B. (Hrsg.): Lexikon des Rechnungswesens, 4. Aufl., München, 1998.

BUZELL, R.D.; GALE, B.T.: Das PIMS-Programm: Strategien und Unternehmenserfolg, Wiesbaden, 1989.

Bromwich, M.: The Case for Strategic Management Accounting: The Role of Accounting Information for Strategy in Competitive Markets, in: Accounting, Organizations and Society, 1990, S. 27 - 46.

Christensen, C.R.; Andrews, K.R.; Bower, J.L.: Business Policy: Text and cases, $3^{\text {rd }}$ ed., Homewood/III. 1973.

CoEnenberg, A.G.: Jahresabschluss und Jahresabschlussanalyse, 17. Aufl., Landsberg am Lech, 2000.

COENENBERG, A.G.: Kostenrechnung und Kostenanalyse, 4. Aufl., Landsberg am Lech, 1999.

CONOVER, W.J.: Practical Nonparametric Statistics, New York, 1971.

DAY, G.S.; MONTGOMERY, D.B.: Diagnosing the Experience Curve, in: JoM, Vol. 47, 1983, No. 2, Page 44 - 58.

ESSER, W.M.: Die Wertkette als Instrument der strategischen Analyse, in: RIEKHOF, H.C. (Hrsg.): Strategieentwicklung, Konzepte - Erfahrungen Fallstudien, Stuttgart, 1994, S. 129 - 151.

FARMeR, R.N.; RICHMAnN, B.M.: Comparative Management and Economic Progress, Homewood III., 1965. 
FISCHER, G.: Strategische Konkurrenzanalyse, in: WIESELHUBER, N.; TÖPFER, A. (Hrsg.): Strategisches Marketing, 2. Aufl., Landsberg, 1986.

FRANZ, K.P.; KAJÜTER, P.: Proaktives Kostenmanagement als Daueraufgabe, in: FRANZ, K.P.; KAJÜTER, P. (Hrsg.): Kostenmanagement, Wettbewerbsvorteile durch systematische Kostensteuerung, Stuttgart, 1997.

Franz, K.P.; KAJÜter, P.: Controlling, in: Busse von Colbe, W.; Coenenberg, A.G.; KAJÜTER, P.; LINNHOFF, U. (Hrsg.): Betriebswirtschaft für Führungskräfte, Stuttgart, 2000 (b).

FRANZ, K.P.; KAJÜteR, P.: Kostenmanagement, in: BuSSE VON COLBE, W.; Coenenberg, A.G.; KAJÜTER, P.; LINNHOFF, U. (Hrsg.): Betriebswirtschaft für Führungskräfte, Stuttgart, 2000 (a).

FRANZEN, W.: Entscheidungswirkung von Kosteninformationen, Frankfurt a.M., 1985.

GÄLWEILER, A.: Strategische Geschäftseinheiten und Aufbauorganisation der Unternehmung, in: ZfO, 48. Jg., 1979, Nr. 5, S. 252 - 260.

GÄLWEILER, A.: Unternehmensplanung, Frankfurt a.M., New York, 1986.

GEIST, M.: Analyse und Politik des Wettbewerbs im Absatzmarkt in betriebswirtschaftlicher Sicht, Mannheim, 1954.

GHEMAWAT, P.: Strategieplanung mit der Erfahrungskurve, in: Harvard Manager, 7. Jg., Heft IV, 1985, S. 33 - 39.

GiBBONS, J.D.; ChAKRABORTI, S.: Nonparametric Statistical Inference, 3.Aufl., New York, 1992.

GLUECK, W.F.: Business Policy and Strategic Management, 3rd ed., Tokio u.a., 1980.

GrAF, J.: Planspiele: Simulierte Realität für den Chef von morgen, Speyer, 1992.

GRANT, J.H.; KING, W.R.: The logic of strategic planing, Boston, Toronto, 1982.

GRIMM, U.: Analyse strategischer Faktoren. Ein Beitrag zur Theorie der strategischen Unternehmensplanung, Wiesbaden, 1983.

GRoss, G.F.: Die Organisation der Konkurrenz-Beobachtung, München, 1974.

GRÜTTER-SETTELE, A.: Verhaltenswirkung von Informationen des externen Rechnungswesens, Augsburg, 1999.

HAHN, D.: Unternehmungsanalyse, in: SZYPERSKI, N. mit Unterstützung von WINAND, U. (HRSG.): HWPlan, Stuttgart, 1989, S. 2075.

HAMMER, R.M.: Strategische Planung und Frühaufklärung, 2. Aufl., München, 1991. 
HAMMER, R.M.: Unternehmensplanung, 6. Aufl., München, Wien, 1995.

HARTUNG, J.: Statistik, Lehr- und Handbuch der angewandten Statistik, 12. Aufl., München, Wien, Oldenbourg, 1999.

HARTUNG, J.; ELPELT, B.: Multivariante Statistik, Lehr- und Handbuch der angewandten Statistik, 6. Aufl., München, Wien, Oldenbourg, 1999.

HAX, A.C.; MAJLUF, N.S.: Strategisches Management - Ein integriertes Konzept aus dem MIT, überarbeitete Studienausgabe, Frankfurt a.M., 1991.

HEDLEY, B.: A Fundamental Approach to Strategy Development, in: HAHN, D.; TAYLOR, B. (Hrsg.): Strategische Unternehmensplanung - Strategische Untemehmensführung, 7. Aufl., Heidelberg, 1997, S. 327 - 341.

HEINEN, K.C.: Es geht in die letzten Runden, MANNESMANN führt, in: HANDELsBLATT, 12./13.03.1999 (a), S. K2.

HeINEN, K.C.: ABB KRAFTWERKE AG gewinnt das Viertelfinale, in: HANDELSBLATt, 23./24.04.1999 (b), S. K2.

HeINEN, K.C.: Die besten Acht kommen ins Finale, in: HANDELSBLATT, 18./19.06. 1999 (c), S. K2.

Heinen, K.C.: Das Finale in Schloss Gracht, Sanacorp Pharmahandel AG geht nach Lissabon, in: HANDELSBLATT, 09./10.07.1999 (d), S. K2.

HEINEN, E.: Industriebetriebslehre, 9. Aufl., Wiesbaden, 1991.

HEINEN, E.: Industriebetriebslehre, 7. Aufl., Wiesbaden, 1983.

HENDERSON, B.D.: Die Erfahrungskurve in der Unternehmensstrategie, 2. Aufl., Frankfurt a.M., 1984.

Hentze, J.; Brose, P.: Unternehmensplanung - Eine Einführung, Bern, Stuttgart, 1985.

Hentze, J.; Brose, P.; Kammel, A.: Unternehmungsplanung, 2. Aufi., Bern, Stuttgart, 1993.

HILL, C.W.L.; JONES, G.R.: Strategic Management. An Integrated Approach, Boston, 1989.

HINTERHUBER, H.H.: Wettbewerbsstrategie, 2. Aufl., Berlin, New York, 1990.

HINTERHUBER, H.H.: Strategische Unternehmungsführung, Band I, Strategisches

Denken, 6. Aufl., Berlin, New York, 1996 (a).

HiNTERHUBER, H.H.: Strategische Unternehmungsführung, Band II, Strategisches Handeln, 6. Aufl., Berlin, New York, 1996 (b).

HINTERHUBER, H.H.; KIRCHEBNER, M.: Die Analyse strategischer Gruppen von Unternehmungen, in: Zeitschrift für Betriebswirtschaft (ZfB), 53. Jg., 1983, S. 854 - 868 . 
HöGSDAL, B.: Planspiele: Einsatz von Planspielen in der Aus- und Weiterbildung, Praxiserfahrungen und bewährte Methoden, Bonn, 1996.

Hofer, C.W.; SCHENDEL, D.: Strategy Formulation: Analytical Concepts, St. Paul, 1978.

HoffmanN, F.: Kritische Erfolgsfaktoren - Erfahrungen in großen und mittelständischen Unternehmen, in: Zeitschrift für betriebswirtschaftliche Forschung (ZfbF), 53. Jg., 1983, Heft 9, S. 854 - 868.

HoffmanN, J.: Die Konkurrenz - Erkenntnisse für die strategische Führung und Planung, in: Töpfer, A.; AfHeld, H. (Hrsg.): Praxis der strategischen Unternehmensplanung, 2. Aufl., Frankfurt a.M., 1986.

HöFLICH, K.: Wettbewerbliche Wirkungen publizierter Jahresabschlüsse, Mainz, 1982.

ILLIG, A.: Konkurrenzanalyse mit Hilfe mulitvarianter Klassifikation, Thun, Frankfurt a.M., 1980.

Hollander, M.; WolfE, D.A.: Nonparametric Statistical Methods, 2. Aufl., New York, 1999.

JOAS, A.: Konkurrenzforschung als Erfolgspotential im strategischen Marketing, Augsburg, 1990.

JOHNSON, G.; SCHOLES, K.: Exploring Corporate Strategie, Englewood Cliffs, 1988.

KAJÜTER, P.: Strategieunterstützung durch Benchmarking, in: WeLGE, M.K.; ALLAHAM, A.; KaJÜTER, P. (Hrsg.): Praxis des Strategischen Managements, Konzepte - Erfahrungen - Perspektiven, Wiesbaden, 2000.

KoLKS, U.: Strategieimplementierung, Wiesbaden, 1990.

KOTLER, P. U.A.: Grundlagen des Marketing, 2. Aufl., München, 1999.

KREIKEBAUM, H.: Strategische Unternehmensplanung, 6. Aufl., Stuttgart, Berlin, Köln, 1997.

KREILKAMP, E.: Strategisches Management und Marketing, Berlin, 1987.

Küpper, H.U.: Kosten und Leistung, in: Busse von Colbe, W.; Pellens, B.

(Hrsg.): Lexikon des Rechnungswesens, 4. Aufl., München, 1998.

Kussmaul, H.: Kostenrechnung, in: Busse von Colbe, W.; Coenenberg, A.G.;

KAJÜTER, P.; LINNHOFF, U. (Hrsg.): Betriebswirtschaft für Führungskräfte, Stuttgart, 2000.

LANGE, B.: Die Erfahrungskurve: Eine kritische Beurteilung, in: ZfbF, 36. Jg., 1984 , S. 229 - 245. 
LeHMANN, E.L.: Nonparametrics - Statistical Methods based on Ranks, San Francisco, 1975.

Lehmann, E.L.: Testing Statistical Hypotheses, 2. Aufl., New York, 1986.

LINDBLOM, C.E.: The intelligence of democracy, New York, London, 1965.

LINDBLOM, C.E.: The science of "muddling through", in: ANSOFF, H.I. (Editor):

Business Strategie, Harmondsworth, 1969.

LINK, U.: Strategische Konkurrenzanalyse im Konsumgütermarketing, Idstein, 1988.

MiCHEL, R.: Know-how der Unternehmensplanung, Heidelberg, Zürich, 1986.

MINTZBERG, H.: Mintzberg on Management, Inside our Strange World of Organizations, New York, 1989.

MintzBeRg, H.; WATERS, J.: Does Decision Get in the Way?, in: Organizational Studies, 11. Jg., 1990, Nr. 1, S. 1 - 6.

NAGEL, K.: Die sechs Erfolgsfaktoren des Unternehmens, Landberg, 1986.

NieschlaG, R.; DichtL, E.; Hörschgen, H.: Marketing, 16. Aufl., Berlin, 1991.

NUTr, P.C.: Selecting Tactics to Implement Strategic Plans, in SMJ, 10. Jg., 1989.

OUINN, J.B.: Strategic Change, Logical Incrementalism, in: QUINN, J.B.; MINTZBERG, H.; JAMES, R.M. (Editor): The Strategy Process, Englewood Cliffs, 1988, S. 94 - 104.

PEARCE, J.A.; Robinson, R.B.: Formulation and Implicatin of Competitive Strategy, 3. Aufl., Homewood III., 1988.

Pettigrew, A.M.: Studying Strategic Choice and Strategic Change, in: Organizational Studies, 11. Jg., 1990, Nr. 1, S. 6 - 11.

PICOT, A.: Strukturwandel und Unternehmensstrategie, in: WiSt, 10. Jg., 1981, S. $527-532$ und S. $536-571$.

PORTER, M.E.: Wettbewerbsstrategie, Methoden zur Analyse von Branchen und Konkurrenten, 10. Aufl., Frankfurt a.M., New York, 1999 (a).

PORTER, M.E.: Wettbewerbsvorteile, Spitzenleistungen erreichen und behaupten, 5. Aufl., Frankfurt a.M., New York, 1999 (b).

PRATT, J.W.; GiBbons, J.D.: Concepts of Nonparametric Theory, New York, 1981.

PÜMPIN, C.: Strategische Führung in der Unternehmenspraxis, Bern, 1980.

RASCHE, H.O.: Praktiker-Checkliste, Bestandsaufnahme und Analyse eines Marktes, Heiligenhaus, 1978.

RÖPER, B.: Die Konkurrenz und ihre Fehlentwicklungen, Berlin, 1952. 
ROGGE, H.J.: Marktforschung, Elemente und Methoden betrieblicher Informationsgewinnung, München, Wien, 1981.

ROHN, W.: Simulation - Praxis im Modell lernen, in: GRAF, J. (Hrsg.): Planspiele: Simulierte Realitäten für den Chef von morgen, Speyer, 1992.

Rosenstiel, L. VON: Motivation von Mitarbeitern, in: Rosenstiel, L. VON; REGNET, E.; DOMSCH, M.E. (Hrsg.): Führung von Mitarbeitern, Handbuch für erfolgreiches Personalmanagement, 4. Aufl., Stuttgart, 1999.

SACHS, L.: Angewandte Statistik, Anwendung statistischer Methoden, 8. Aufl., Berlin, Heidelberg, New York, 1997.

SAUERMANN, H.: Die experimentelle Wirtschaftsforschung an der Universität Frankfurt a.M., in: SAUERMANN, H. (Hrsg.): Beiträge zur experimentellen Wirtschaftsforschung, Band II, Tübingen, 1970.

SAUERMANN, H.; Selten, R.: Zur Entwicklung der experimentellen Wirtschaftsforschung, in: SAUERMANN, H. (Hrsg.): Beiträge zur experimentellen Wirtschaftsforschung, Band I, Tübingen, 1967.

SAMMON, W.L.: Assessing the Competition: Business Intelligence for Strategic Management, in: EPHMRA/EsOMAR SEMINAR (Hrsg.): Pharmaceutical Competitor Analysis: A Guide for Marketing Researchers, Amsterdam, 1985.

SCHÄFER, E.: Grundlagen der Marktforschung, Marktuntersuchung und Marktbeobachtung, 4. Aufl., Köln, Opladen, 1966.

SCHEYÖGG, G.: Unternehmensstrategie, Berlin, New York, 1984.

SCHMALENBACHGESELLSCHAFT, Arbeitskreis "Langfristige Unternehmensplanung", Schmalenbachs Zeitschrift für betriebswirtschaftliche Forschung, ZfbF, Jg. 29, 1977.

SCHNEIDER, D.: Allgemeine Betriebswirtschaftslehre, 2. Aufl., München, Wien, 1985.

SCHNEIDER, D.: Erste Schritte zu einer Theorie der Bilanzanalyse, in: Die Wirtschaftsprüfung, 42. Jg., 1989, S. 633 - 642.

SCHOLZ, C.: Strategisches Management, Ein integrativer Ansatz, Berlin, New York, 1987.

SIMMONDS, K.: Strategisches Management Accounting, in: Controlling, 1989, S. 264. - 269.

STEIN, H.G.: Kostenführerschaft als strategische Erfolgsposition, in: HENZLER, H. (Hrsg.): Handbuch Strategische Führung, Wiesbaden, 1988, S. 397 - 426. STEINER, G.A.: Top Management Planing, London, 1969. 
SteInER, G.A.; Miner, J.B.: Management Policy and Strategy, 2nd ed., New York, 1982.

SteinmanN, H.; Guthunz, U.; HASSElBERg, F.: Kostenführerschaft und Kostenrechnung, in: MÄNNEL, W. (Hrsg.): Handbuch Kostenrechnung, Wiesbaden, 1992.

THOMAS, J.G.: Strategic Management, New York, 1988.

THOMmen, J.P.; ACHLEITNER, A.K.: Allgemeine Betriebswirtschaftslehre, 2. Aufl., Wiesbaden, 1998.

TIMmermanN, A.: Evolution des strategischen Managements, in: HenZLER, $H$. (Hrsg.): Handbuch Strategische Führung, Wiesbaden, 1988.

TÖPFER, A.: Planungs- und Kontrollsysteme industrieller Unternehmungen, Berlin, 1976.

TOURANGEAU, K.W.: Strategy management, New York, u.a., 1981.

TROBMANN, E.: Internes Rechnungswesen, in: CORSTEN, H.; REISS, M. (Hrsg.): Betriebswirtschaftslehre, 3. Aufl., München, 1999.

ULRICH, H.: Unternehmungspolitik, 3. Aufl., Bern, Stuttgart, 1990.

UNIVERSITÄTSSEMINAR DER WIRTSCHAFT: MARGA-Handbuch 1999, Erftstadt, 1998.

Voss, A.: Trends in der Management-Entwicklung: Unveröffentlichte wissenschaftliche Untersuchung im Rahmen eines Forschungsprojektes an der Technischen Universität Berlin, Institut für Betriebswirtschaftslehre, unter der Leitung von PROF. DR. D. GEBERT, Berlin, 1999.

VosS, A.; HÄRING, K.; Welge, M.K.: Der Wettlauf mit dem Wandel: Management-Entwicklung im Umbruch, in: WELGE, M.K.; HÄRING, K.; VosS, A. (Hrsg.): Management Development: Praxis, Trends, Perspektiven, Stuttgart, 2000.

WACKER, P.A.: Die Erfahrungskurve in der Unternehmungsplanung - Analyse und empirische Überprüfung, München, 1980.

WEBER, K.: Jahresabschlussanalyse Deutscher und Schweizerischer Rückversicherungsunternehmen, Köln, 1987.

WELGE, M.K.: Unternehmungsführung, Band 1: Planung, Stuttgart, 1985.

Welge, M.K.; Al-Laham, A.: Planung, Prozesse - Strategien - Maßnahmen, Wiesbaden, 1992.

WELGE, M.K.; AL-LAHAM, A.: Strategisches Management, Grundlagen - Prozess - Implementierung, 2. Aufl., Wiesbaden, 1999. 
WELGE, M.K.; AMSHOFF, B.: Neuorientierung der Kostenrechnung zur Unterstützung der strategischen Planung, in: FRANZ, K.P.; KAJÜTER, P. (Hrsg.): Kostenmanagement: Wettbewerbsvorteile durch systematische Kostensteuerung, Stuttgart, 1997.

Wieselhuber, N.: Phasen und Prozess der strategischen Planung, in: TöPfER, A.; AfHELDT, H. (Hrsg.): Praxis der strategischen Unternehmensplanung, 2. Aufl., Frankfurt a.M., 1986.

WILD, J.: Grundlagen der Unternehmensplanung, 4. Aufl., Opladen, 1982.

WITTMANN, W.: Unternehmung und unvollkommene Information, Köln, Opladen, 1956.

WöHE, G.: Einführung in die allgemeine Betriebswirtschaftslehre, 19. Aufl., München, 1996.

Ohne Verfasser: Im Team trainieren für den Manager-Job, MANNESMANN und USW kürten die Sieger im Unternehmensplanspiel THE BOARD, in: DIE WELT, 31.07.1999, S. BR 1. 


\section{Beiträge zum Controlling}

\section{Herausgegeben von Wolfgang Berens}

Band 1 Wolfgang Berens / Joachim Strauch: Due Diligence bei Unternehmensakquisitionen - eine empirische Untersuchung. Unter Mitarbeit von Thorsten Behrens und Julia Lescher. 2002.

Band 2 Andreas Siemes: Marktorientierte Kreditrisikobewertung. Eine empirische Untersuchung mittels Künstlicher Neuronaler Netze. 2002.

Band 3 Karl Christoph Heinen: Die Berücksichtigung von Kosten in der Konkurrenzanalyse. 2002. 


\section{Preis-Kosten-Schere}

\section{Ein quantitatives Instrument zur Beschreibung von Wettbewerbsdynamiken im Rahmen des Controllings}

Frankfurt/M., Berlin, Bern, Bruxelles, New York, Oxford, Wien, 2000. LXIII, 251 S., 57 Abb., 21 Tab.

Europäische Hochschulschriften: Reihe 5, Volks- und Betriebswirtschaft. Bd. 2695

ISBN 3-631-37319-8 · br. $€ 50.10$ *

Die Preis-Kosten-Schere ist ein Controlling-Instrument zur Beschreibung von Wettbewerbsdynamiken, das komplexe Marktmechanismen und deren Wirkung auf das einzelne Unternehmen durch eine einfach durchzuführende, aber aussagekräftige und sehr gut zu kommunizierende Analyse darstellt. Sie hilft dem einzelnen Unternehmen, die aus externen Marktentwicklungen resultierenden Chancen und Risiken systematisch zu analysieren und daraus den Umfang erforderlicher Anpassungsmaßnahmen zu ermitteln. Die PreisKosten-Schere wird in der betriebswirtschaftlichen Literatur lediglich in ihren Grundzügen beschrieben. Daher besteht das Ziel der Arbeit darin, ein ganzheitliches theoretisches Konzept für die Preis-Kosten-Schere zu erarbeiten und ihre möglichen Anwendungsfälle in der Praxis aufzuzeigen.

Aus dem Inhalt: Einordnung der Preis-Kosten-Schere in den betriebswirtschaftlichen Kontext - Erarbeitung eines theoretischen Konzepts der PreisKosten-Schere unter vereinfachten Produkt- und Markteigenschaften - Sukzessive Aufhebung der Vereinfachungen mit dem Ziel der Erweiterung des Anwendungsspektrums des Instrumentes - Verdeutlichung der theoretischen Konzepte am Beispiel zweier empirischer Fälle

Frankfurt/M · Berlin · Bern · Bruxelles · New York · Oxford · Wien

Auslieferung: Verlag Peter Lang AG

Jupiterstr. 15, CH-3000 Bern 15

Telefax (004131) 9402131

*inklusive der in Deutschland gültigen Mehrwertsteuer

Preisänderungen vorbehalten

Homepage http://www.peterlang.de 